Consortium for Advanced Simulation of LWRs

\title{
CTF User's Manual
}

Version 4.2

R. Salko ${ }^{1}$, M. Avramova ${ }^{2}$, A. Wysocki ${ }^{1}$, J. Hu$^{1}$, A.

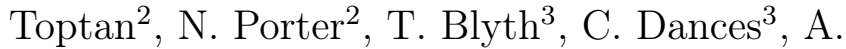
Gomez $^{3}$, C. Jernigan ${ }^{4}$, and J. Kelly ${ }^{5}$

${ }^{1}$ Oak Ridge National Laboratory

${ }^{2}$ North Carolina State University

${ }^{3}$ The Pennsylvania State University

${ }^{4}$ Holtec International

${ }^{5}$ US Nuclear Regulatory Commission

$9 / 14 / 2020$

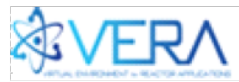

VERA QA Document - This document was prepared under the ORNL VERA Quality Assurance Program in accordance with procedure VERA-QA-003. The OFFICIAL COPY of this document is the electronic version in the VERA Documents Repository. Before using a printed copy, verify that it is the most current version by checking the Revision ID against the electronic version.

Approved for Public Release
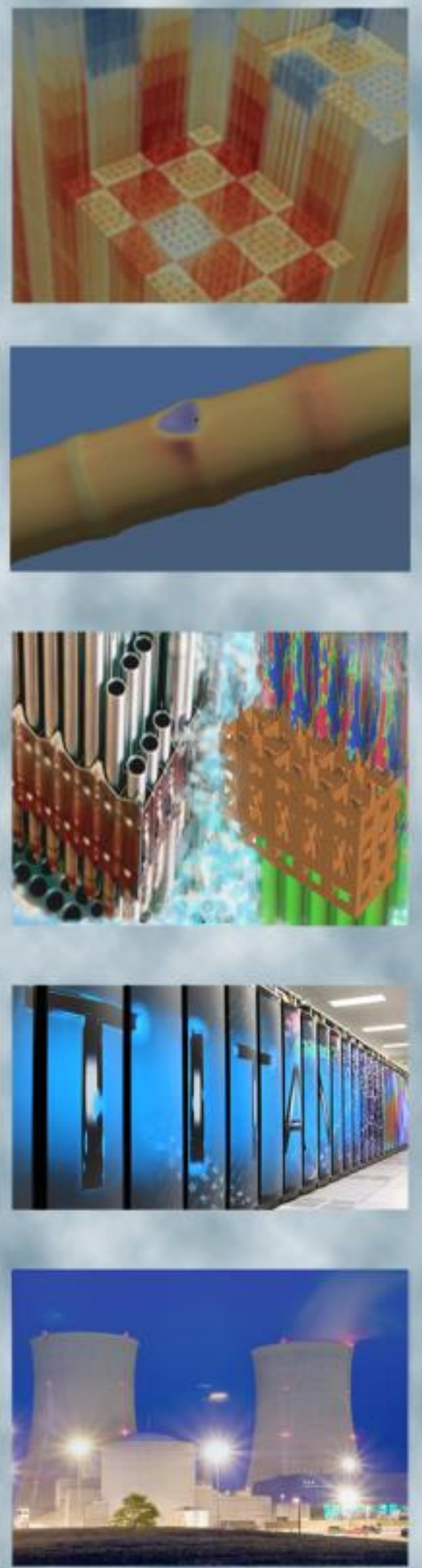


\title{
Revision Log
}

\begin{tabular}{rrrr}
\hline Revision & Date & Affected Pages & Revision Description \\
\hline 0 & $3 / 24 / 19$ & All & Initial Release \\
1 & $11 / 8 / 19$ & All & Updates for 4.1 release \\
2 & $9 / 14 / 20$ & All & Updates for 4.2 release
\end{tabular}

\section{Document pages that are:}

\author{
Export Controlled: None \\ IP/Proprietary/NDA Controlled: None \\ Sensitive Controlled: None \\ Unlimited: All
}

This document was prepared as an account of work sponsored by an agency of the United States Government. Neither the United States Government nor any agency thereof, nor any of their employees, makes any warranty, express or implied, or assumes any legal liability or responsibility for the accuracy, completeness, or usefulness of any information, apparatus, product, or process disclosed, or represents that its use would not infringe privately owned rights. Reference herein to any specific commercial product, process, or service by trade name, trademark, manufacturer, or otherwise, does not necessarily constitute or imply its endorsement, recommendation, or favoring by the United States Government or any agency thereof. The views and opinions of authors expressed herein do not necessarily state or reflect those of the United States Government or any agency thereof. 


\section{The North Carolina State University}

DEPARTMENT OF NuClEAR ENGINEERING

Reactor Dynamics and Fuel Modeling Group

\section{NC STATE UNIVERSITY}

\section{CTF User's Manual}

Version 4.2

September 28, 2020 


\section{CTF User's Manual}

Version 4.2

\section{Approvals:}

Robert Salko, CTF Product Software Manager

Belgacem Hizoum, Independent Reviewer
Date

Date 


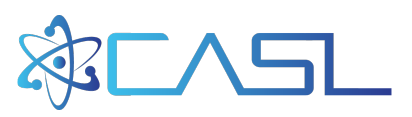

CTF User's Manual

\section{Executive Summary}

This document describes how a user should go about using the CTF pre-processor tool to create an input deck for modeling rod-bundle geometry in CTF. The tool was designed to generate input decks in a quick and less error-prone manner for CTF. The pre-processor is a completely independent utility, written in Fortran, that takes a reduced amount of input from the user. The information that the user must supply is basic information on bundle geometry, such as rod pitch, clad thickness, and axial location of spacer grids - the pre-processor takes this basic information and determines channel placement and connection information to be written to the input deck, which is the most time-consuming and error-prone segment of creating a deck. Creation of the model is also more intuitive, as the user can specify assembly and water-tube placement using visual maps instead of having to place them by determining channel/channel and rod/channel connections. As an example of the benefit of the pre-processor, a quarter-core model that contains 500,000 scalar-mesh cells was read into CTF from an input deck containing 200,000 lines of data. This 200,000 line input deck was produced automatically from a set of pre-processor decks that contained only 300 lines of data. 


\section{Contents}

Executive Summary $\ldots \ldots \ldots \ldots \ldots \ldots \ldots \ldots \ldots \ldots \ldots$

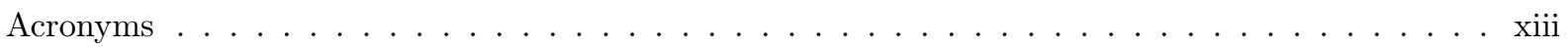

1 Input Manual $\quad 1$

1.1 General Remarks . . . . . . . . . . . . . . . . . . . . 1

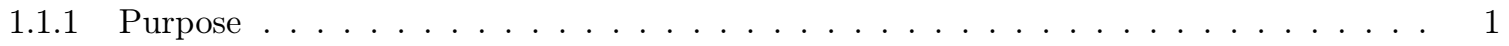

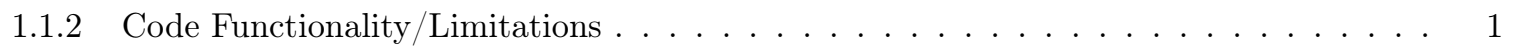

1.1 .3 Code installation . . . . . . . . . . . . . . . . . . . . . . . . 1

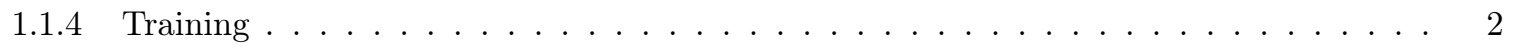

1.1.5 Problem Reporting Methods . . . . . . . . . . . . . . . . . . 2

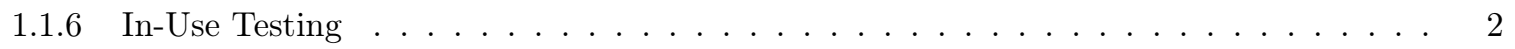

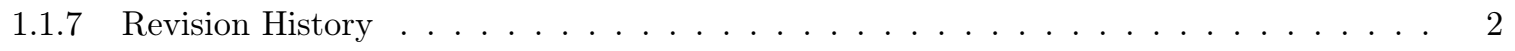

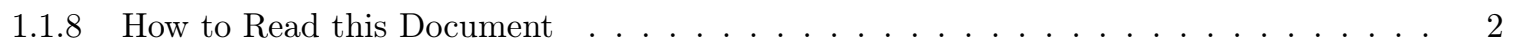

1.1 .9 Standard CTF Input File $\ldots \ldots \ldots \ldots \ldots$

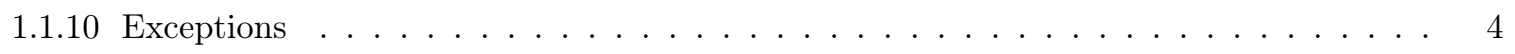

2 Main Problem Control Data $\quad 5$

2.1 Units of Physical Quantities in Input and Output . . . . . . . . . . . . . . . 5

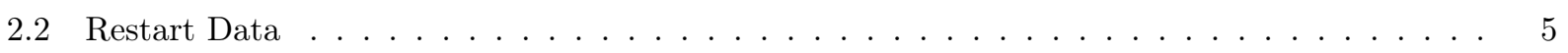

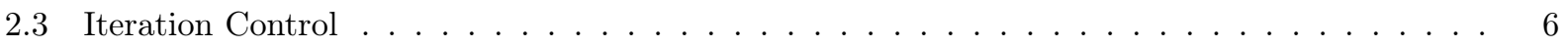

Consortium for Advanced Simulation of LWRs vi CASL-U-2019-1885-002 


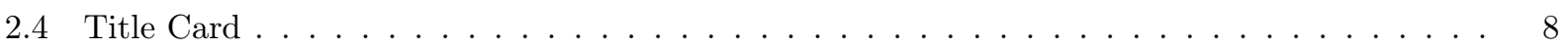

$\begin{array}{llr}3 & \text { Card Group } 1 & 9\end{array}$

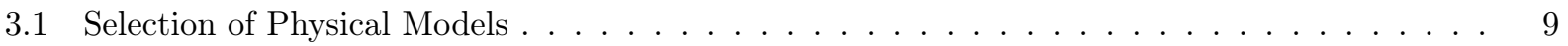

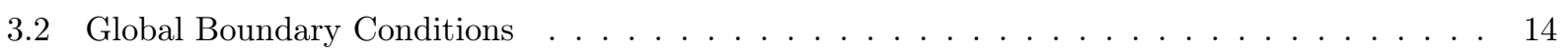

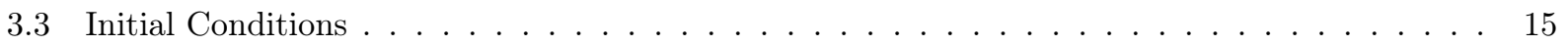

3.4 Custom Friction Model Input $\ldots \ldots \ldots \ldots$

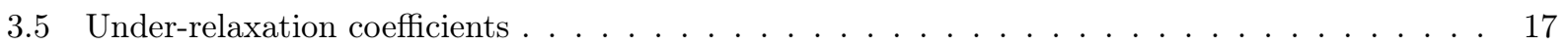

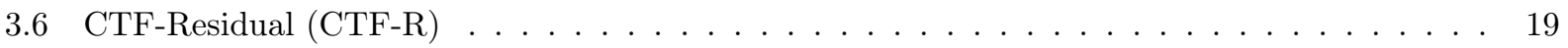

3.7 Onset of nucleate boiling $(\mathrm{ONB}) \ldots \ldots \ldots \ldots \ldots \ldots$

3.8 Notes on Card Group $1 \ldots \ldots \ldots \ldots \ldots$

4 Card Group 2 21

5 Card Group $3 \quad 26$

6 Card Group $4 \quad 33$

$\begin{array}{llr}7 & \text { Card Group } 5 & 38\end{array}$

$\begin{array}{lll}8 & \text { Card Group } 6 & 40\end{array}$

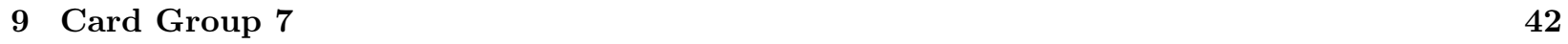

10 Card Group $8 \quad 53$

$10.1 \operatorname{Rod}$ Geometry Data . . . . . . . . . . . . . . . . . . . . . 57

10.2 Unheated Conductor Data . . . . . . . . . . . . . . . . . . . . . . 61

10.3 Rod Temperature Initialization Tables $\ldots \ldots \ldots$

10.4 Radiation Initialization Tables $\ldots \ldots \ldots \ldots \ldots$

10.5 Radiation Channel Orientation Array _ . . . . . . . . . . . . . . . . . 67

10.6 Cavity to Cavity Radiation Weighting Factor $\ldots \ldots \ldots \ldots \ldots \ldots$ 
10.7 Radiation Location Type Information $\ldots \ldots \ldots \ldots \ldots \ldots$

$\begin{array}{ll}11 \text { Card Group } 9 & 77\end{array}$

11.1 Nuclear Fuel Geometry Types . . . . . . . . . . . . . . . . . . . . . . . 80

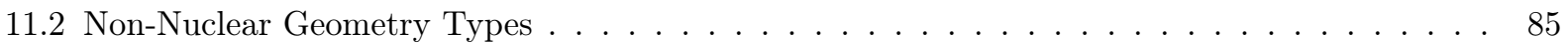

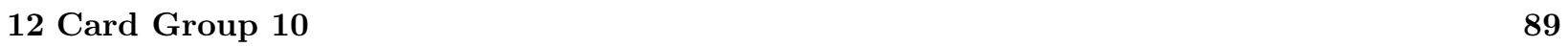

$\begin{array}{ll}13 \text { Card Group } 11 & 91\end{array}$

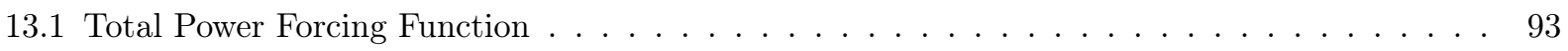

13.2 Gap Conductance Forcing Function . . . . . . . . . . . . . . . . . . . . . 94

13.3 Radial Power Profile Forcing Function . . . . . . . . . . . . . . . . . . . . 94

14 Card Group $12 \quad 95$

$\begin{array}{ll}15 \text { Card Group } 13 & 98\end{array}$

$\begin{array}{ll}16 \text { Card Group } 14 & 107\end{array}$

16.1 Legacy Card Group $14 \ldots \ldots$. . . . . . . . . . . . . . . . . . . 107

16.2 New Card Group $14 \ldots \ldots$. . . . . . . . . . . . . . . . . . . 112

$\begin{array}{ll}17 \text { Card Group } 15 & 118\end{array}$

$\begin{array}{lr}18 \text { Card Group } 16 & 120\end{array}$

$\begin{array}{ll}19 \text { Card Group } 17 & 121\end{array}$

20 Card Group $18 \quad 128$

$\begin{array}{lr}21 \text { Card Group } 19 & 130\end{array}$

22 Output Manual $\quad 136$

Consortium for Advanced Simulation of LWRs viii CASL-U-2019-1885-002 


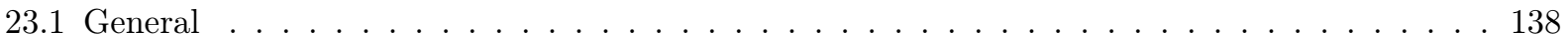

23.2 Specification of the Geometry Data . . . . . . . . . . . . . . . . . 141

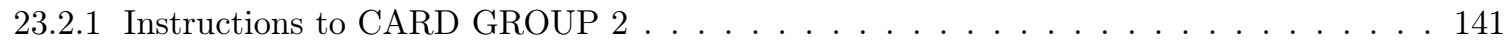

23.2 .2 Instructions to CARD GROUP $3 \ldots \ldots \ldots \ldots \ldots \ldots$

23.2 .3 Instructions to CARD GROUP $4 \ldots \ldots \ldots \ldots \ldots \ldots$

23.2.4 Instructions to CARD GROUP 5 and $6 \ldots \ldots \ldots \ldots \ldots \ldots \ldots \ldots \ldots \ldots \ldots \ldots$

23.2.5 Instructions to CARD GROUP $7 \ldots \ldots \ldots \ldots \ldots \ldots$

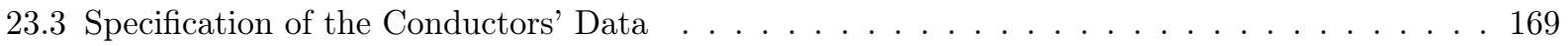

23.3.1 Instructions to CARD GROUP $8 \ldots \ldots \ldots \ldots \ldots$

23.3.2 Instructions to CARD GROUP $9 \ldots \ldots \ldots \ldots \ldots \ldots \ldots$

23.3 .3 Instructions to CARD GROUP $10 \ldots \ldots \ldots \ldots \ldots \ldots$

23.4 Specification of the Initial and Boundary Conditions _ . . . . . . . . . . . . . 182

23.4.1 Instructions to CARD GROUP $1 \ldots \ldots \ldots \ldots$. . . . . . . . . . . . . . . . .

23.4 .2 Instructions to CARD GROUP $11 \ldots \ldots \ldots \ldots \ldots \ldots$

23.4 .3 Instructions to CARD GROUP $13 \ldots \ldots \ldots \ldots \ldots \ldots$

23.4.4 Boron Tracking and Precipitation Modeling . . . . . . . . . . . . . . . . . 194

23.4.4.1 Application of the Boron Tracking/Precipitation Model . . . . . . . . . . . . 194

23.4.4.2 Example of Card Group 1 and Card Group 13 (Boron Tracking/Precipitation Model $\ldots$. . . . . . . . . . . . . . . . . . . . . . 195

23.5 Turbulent Mixing and Void Drift Modeling . . . . . . . . . . . . . . 196

23.5.1 Instructions to CARD GROUP $12 \ldots \ldots \ldots \ldots \ldots \ldots \ldots$

23.5.2 Instructions to CARD GROUP $12 \ldots \ldots \ldots \ldots \ldots$

23.6 Results Reporting . . . . . . . . . . . . . . . . . . . . . . . . . 203

23.6.1 Instructions to CARD GROUP 14 (Legacy) . . . . . . . . . . . . . . . . . . . 204

23.7 Main Problem Control and Time Domain Data . . . . . . . . . . . . . . . . . . . 204

23.7.1 Instructions to Input of Main Problem Control Data . . . . . . . . . . . . . . . 205 
23.7.2 Instructions to Input of Time Domain Data . . . . . . . . . . . . . . 205

23.7.3 Instructions to Preparation of Input Files for Restart Calculations . . . . . . . . . . 206

A Calculation Notes and the 3x3 GE Experiments Input Decks

A.1 GE 3x3 Experimental Parameters . . . . . . . . . . . . . . . . . . . . . . . 212

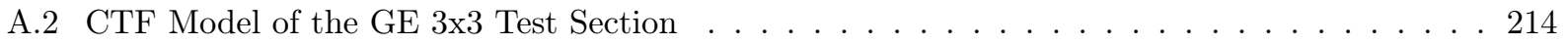

A.3 CTF Input Deck for the GE $3 \times 3$ Test Point $2 \mathrm{~B} 2 \ldots \ldots \ldots \ldots \ldots \ldots \ldots$

Bibliography 


\section{List of Figures}

4.1 Definition of the X, Y, XSIZ, and YSIZ terms for Card 2.2 for a single assembly, plus a suggestion of where the origin can be placed . . . . . . . . . . . . . . . . . . . . . . 23

4.2 Definition of the $(0,0)$ location and $\mathrm{X}$ and $\mathrm{Y}$ for core geometry . . . . . . . . . . . . . 24

10.1 Radiation Geometry Type 1 . . . . . . . . . . . . . . . . . . . . 67

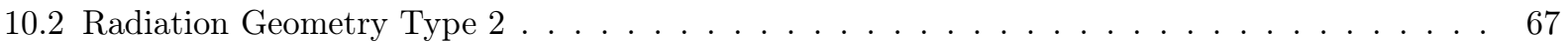

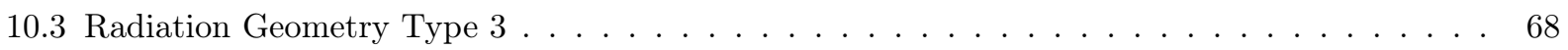

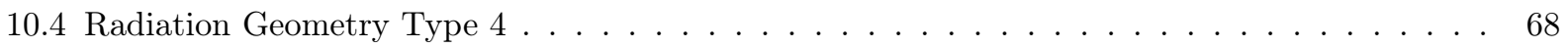

10.5 Radiation Geometry Type $5 \ldots$. . . . . . . . . . . . . . . . . . . 68

10.6 Radiation Geometry Type $6 \ldots \ldots$. . . . . . . . . . . . . . . . . . 68

10.7 Radiation Geometry Type 10 . . . . . . . . . . . . . . . . . . . . . . 69

10.8 Radiation Geometry Type 11 . . . . . . . . . . . . . . . . . . . . . . . 69

10.9 Radiation Geometry Type 12 . . . . . . . . . . . . . . . . . . . . . . . 69

10.10Radiation Geometry Type 13 . . . . . . . . . . . . . . . . . . . . . . 69

10.11Radiation Geometry Type 14 . . . . . . . . . . . . . . . . . . . . . 70

10.12Radiation Geometry Type 15 . . . . . . . . . . . . . . . . . . . . . 70

10.13Radiation Geometry Type 16 . . . . . . . . . . . . . . . . . . . . 70

10.14Radiation Geometry Type 17 . . . . . . . . . . . . . . . . . . . . 70

19.1 Core map of $3 \times 3$ assemblies . . . . . . . . . . . . . . . . . . . . . . . . . . . . . . 124 
23.1 Basic mesh cell . . . . . . . . . . . . . . . . . . . . . . . . . 139

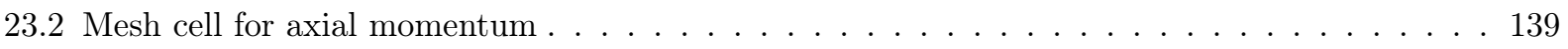

23.3 Mesh cell for transverse momentum . . . . . . . . . . . . . . . . . . . . 140

23.4 Basic Subchannel . . . . . . . . . . . . . . . . . . . . . . . . . . . . 140

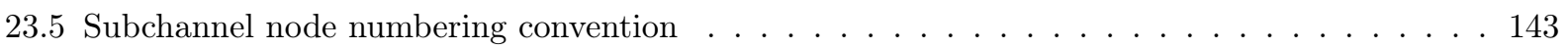

23.6 Subchannel connections at section boundaries allowed by the subchannel splitting logic . . . . 144

23.7 Typical configuration for convection of transverse momentum between sections . . . . . . 147

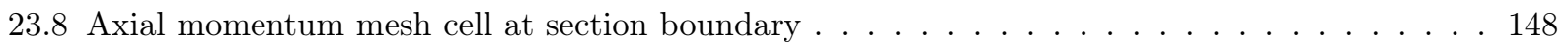

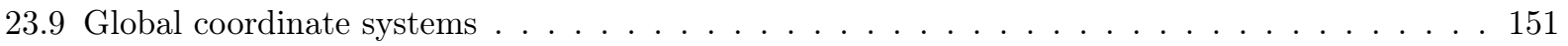

23.10Axial momentum mesh cell at section boundary . . . . . . . . . . . . . . . 152

23.11Lateral pipe flow case with axial mass flow inlet and pressure outlet $\ldots \ldots \ldots$

23.12 Convection of transverse momentum by an orthogonal transverse velocity . . . . . . . 154

23.13Diagram of the variable axial node length $\ldots \ldots \ldots \ldots \ldots \ldots \ldots \ldots \ldots \ldots \ldots$

23.14Allowable vertical connections between subchannels at section boundaries $\ldots \ldots$. . . . 157

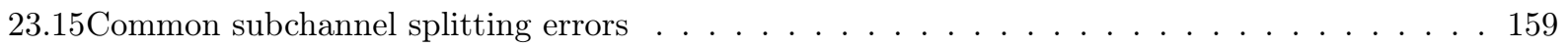

23.16Common subchannel splitting errors . . . . . . . . . . . . . . . . 161

23.17Examples of axial variation in continuity and momentum area and wetted perimeter of a

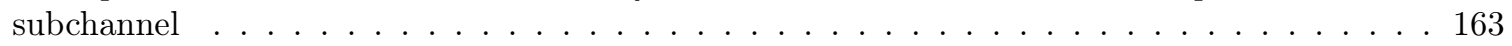

23.18Example of subchannels with local form losses due to spacer grids $\ldots \ldots \ldots 6$

23.19Example of rod surface numbering in CARD $7.5 \ldots \ldots \ldots \ldots \ldots \ldots \ldots \ldots$

$23.203 \times 3$ BWR Bundle . . . . . . . . . . . . . . . . . . . . . . . 172

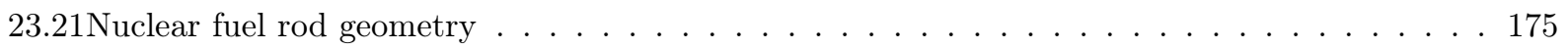

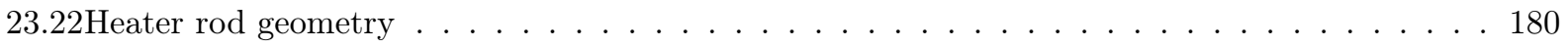

23.23 Nuclear fuel $\operatorname{rod}$ power profile $\ldots \ldots \ldots \ldots \ldots \ldots$

23.24 Heat input over one fluid node $\ldots \ldots \ldots \ldots \ldots \ldots$

23.25 Heater rod crossing section boundaries $\ldots \ldots \ldots \ldots \ldots \ldots$

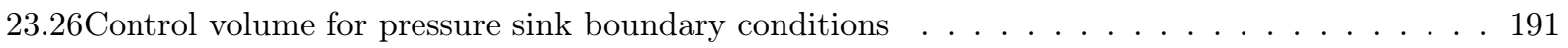


A.1 Cross section of the CTF model of the GE $3 \times 3 \operatorname{rod}$ bundle . . . . . . . . . . . . . . . . 214 


\section{Acronyms}




\section{Input Manual}

\subsection{General Remarks}

\subsubsection{Purpose}

This document provides detailed instructions for creating or modifying the native CTF input file, which can be used to define model geometry and operating conditions and can be run with the standalone CTF executable. The summary of CTF documentation can be found in the CTF Software Management Plan.

\subsubsection{Code Functionality/Limitations}

CTF was developed for specific intended applications. The CTF Verification and Validation Manual states high-level requirements and documents tests that demonstrate how it meets these requirements. The CTF Software Requirements, Test Plan, and Test Report document states low-level requirements and documents the tests that ensure it meets these requirements. The user should assume that any desired functionality that is not clearly stated in the high- or low-level requirements lists is not a supported function of CTF.

Note that CTF does include more features than are necessary to support its intended applications. These features are considered research features, and their quality/correctness is not guaranteed due to limited testing. Any features that are not stated in the aforementioned requirements lists should be considered to be experimental, and their use is cautioned.

Additional limitations include code input limitations and code modeling limitations. Code input limitations are stated in this manual for each possible CTF input. Code modeling limitations (which generally refer to closure model range of validity) are stated in the CTF Theory Manual or in the closure model's referenced documentation when available.

\subsubsection{Code installation}

Code installation instructions are covered in the VERA release notes that are provided for the specific version of CTF being distributed. It is suggested that access to the installed software be limited to trained and qualified users. 


\subsubsection{Training}

The intended CTF user is one who is familiar with computational thermal-hydraulics codes. Prior to using CTF, the user should read through the CTF documentation suite identified in the CTF Software Management Plan. ORNL and the CTF User Group do periodically hold CTF training courses and can be contacted at salkork@ornl.gov for further information.

\subsubsection{Problem Reporting Methods}

The problem reporting method is described in "VERA-QA-010: VERA Problem Reporting and Corrective Action".

\subsubsection{In-Use Testing}

Upon building CTF, the user can execute the code test suite by running "ctest" in the build directory. This will execute all of the automated CTF tests that were enabled during code configuration time.

\subsubsection{Revision History}

The deck version is specified using the PPV flag on Card 1.1. In general, you should always use the latest version of the CTF input deck in order to make sure your model is treated correctly in CTF. This feature was added to retain backwards compatibility for older input decks. When an input deck is created with the CTF pre-processor, the latest version will always be used. Table 1 provides the revision history of the CTF input deck. The first column gives the version number of the deck and the second columns gives a description of what changed since the previous version of the input deck.

\subsubsection{How to Read this Document}

This document describes how to make a CTF input deck. A CTF input deck is organized into Card Groups and Cards. A Card Group is a collection of Cards. A Card is defined as a line of input. Each Card may contain multiple data. A Card is terminated by making a new line.

This document has been organized so that each Card Group is discussed in its own dedicated chapter. Each card is discussed in its own dedicated section. The data in each card are discussed in their own block. The block gives information about the data, including the number of the input, the title, a description of the meaning of the data, units, data type, and so on. An example block is shown below to discuss the meaning of each entry in the block. 
Table 1: Revision history of the CTF input deck

\begin{aligned} & \hline Version Remarks \\ & \hline 0 Original CTF input deck version \\ & 1 $\begin{array}{l}\text { Added ability to have more ghost entities (rods, gaps, channels) for parallel } \\ \text { models. All parallel decks should be at least Version } 1 \text { to work correctly }\end{array} \\ & 2 \begin{array}{l}\text { Added new term, SYMROD, on Card } 8.2 \text {, which allows the user to specify } \\ \text { if the rod is a partial rod (if a symmetry line runs through the rod). All }\end{array} \\ & \begin{array}{l}\text { symmetry models should be at least version } 2 . \\ \text { Added new friction correlations that allow for modeling surface roughness } \\ \text { effects as well as for user to input custom friction factor correlation. These }\end{array} \\ &$ new correlations require surface roughness to be specified or correlation co- \\ & efficients to be specified, which required a change to the input deck format. \end{aligned}

\begin{tabular}{ll|l|c|c}
$\begin{array}{l}\text { \{Card Group Num- } \\
\text { ber }\} .\{\text { Card } \\
\text { ber }\}\end{array}$ & Num- & Parameter Name $\}$ & $\begin{array}{c}\text { [Physical units of the } \\
\text { parameter in SI }\}]\end{array}$ & $\begin{array}{c}{[\text { Physical units of the }} \\
\text { parameter in US }\}]\end{array}$ \\
\hline
\end{tabular}

\{Description of the parameter. Possible values.\}

\begin{tabular}{l|l} 
\{Data type $\}$ & \{Required or Optional
\end{tabular}

\{Range of validity. If "- " is entered, then it means see description for valid options, and if "Any" is entered it means ther

\subsubsection{Standard CTF Input File}

- The CTF executable can be run with the input file name as an argument on the command line. If no file name is provided, the code will look for a file called "deck.inp" by default. For all options, run the CTF executable with the "-help" argument.

- Input files are read in a free-formatted way only

- Because of the free-formatted input structure each of the parameters described below has to be specified in the input file (as dummy parameters, in case they are not used in the code)

- Lines must not be longer than $\mathbf{2 0 0}$ characters. Otherwise the remaining characters are truncated, which could cause reading errors 
CTF is intended to be built and run on a Linux-based operating system. For a serial run, only one input file is required. This can be called the default name, "deck.inp", or a custom name "〈name〉.inp". If the default naming convention is used, the code may be run either as:

$\$\{\mathrm{CTF}\}$

or

$\$\{\mathrm{CTF}\}$ deck.inp

or

$\$\{\mathrm{CTF}\}$ deck

If the custom naming convention is used, the code may be run either as:

$\$\{C T F\}\langle$ name $\rangle$. inp

or

$\$\{C T F\}\langle$ name〉

Additional options exist for running CTF. These can be found by executing CTF with the -help command:

$\$\{\mathrm{CTF}\}-$ help

\subsubsection{Exceptions}

CTF will produce warning or error messages for unexpected or erroneous input. A warning message will be presented to the user when the input is not expected or not consistent, but when the code simulation may still continue using some default behavior. An error message is presented when code simulation cannot continue due to invalid input. Code execution is terminated with an error code when an error is encountered. Exception messages will be printed to the standard error stream and the log file and will be clearly marked with a banner identifying the message as an error or warning. 


\section{Main Problem Control Data}

This card group is read by subroutines INPUT and COBRAI.

\subsection{Units of Physical Quantities in Input and Output}

\begin{tabular}{|c|c|c|c|}
\hline INPUT.1 & ICOBRA & {$[-]$} & {$[-]$} \\
\hline \multicolumn{4}{|c|}{ Units used in input and output files: } \\
\hline \multicolumn{4}{|c|}{0 - US input / SI output } \\
\hline \multicolumn{4}{|c|}{1 - SI input / SI output } \\
\hline \multicolumn{4}{|c|}{2 - US input / US output } \\
\hline \multicolumn{4}{|c|}{3 - SI input / US output } \\
\hline Integer & Required & & \\
\hline - & & & \\
\hline
\end{tabular}

\section{$2.2 \quad$ Restart Data}

Note that the CTF restart feature is currently not functional. All following inputs must be set so the restart feature is not being utilized. 


\begin{tabular}{|l|l|c|}
\hline INPUT.2 & INITIAL & {$[-]$} \\
\hline Vessel initialization option: \\
1 - Initial start \\
Initial values are specified in Card 1.2 \\
2 - Full restart (not currently working) \\
Variable arrays are filled with data obtained from the restart file 'deck.crs'. Operating condi- \\
tions, boundary conditions, and time domain data can be changed for the restart fun. \\
- Simple restart (not currently working) \\
Variable arrays are filled with data obtained from the restart file 'deck.crs'. Only time domain \\
data can be changed for the restart run. \\
\hline Integer \\
\hline-
\end{tabular}

If INITIAL is set equal to 2 (full restart), then the restart file:

- must contain Card Groups:

INPUT.1, INPUT.2, INPUT.3, COBRA.1, 15

- may contain Card Groups:

$1,11,12,13,14$

- must not contain Card Groups:

$2,3,4,5,6,7,8,9,10$

\begin{tabular}{|c|c|c|c|}
\hline INPUT.2 & DUMPF & {$[-]$} & {$[-]$} \\
\hline \multicolumn{4}{|c|}{ Flag for restart file generation: } \\
\hline \multicolumn{4}{|c|}{0 - No restart file is generated } \\
\hline \multicolumn{4}{|c|}{$\begin{array}{l}1 \text { - Restart file 'deck.cdm' is generated in time inteverals of DMPINT (Card 15.1) and also at the end } \\
\text { of the calculation }\end{array}$} \\
\hline Integer & Required & & \\
\hline
\end{tabular}

\subsection{Iteration Control}

The following three controls were implemented to allow the user to modify solution tolerances and iteration caps on the CTF numerical solution algorithm. However, not all of these controls are currently functional in the code. 
EPSO will only have an effect if PETSc is used to solve the pressure matrix (ISOL $=5$ or 7 ). In this case, EPSO is defined as the relative tolerance at which the iterative pressure matrix solution can be stopped for a given outer iteration of CTF. For steady-state simulations, it is permissible to set this value to a relatively high value. This may speed up the pressure matrix solve substantially at the cost of slightly larger error in the intermediate calculated pressure distribution; however, the intermediate pressure solution is not of great importance in a steady-state solution. At the end of the simulation, the solution will arrive at the same pressure distribution as if a lower relative tolerance were used. For transient simulations, the intermediate CTF outer iteration solutions are important, and a lower relative tolerance should be used. The PETSc default tolerance of $1 \cdot 0 \cdot 10^{-5}$ is recommended for transients. A default value for steady-state solutions cannot be recommended, as an in-depth analysis of this parameter effect on solution time has not been performed. Furthermore, the optimal value will change with problem size and number of solution domains (larger problems will spend more time in the pressure matrix solve due to increased communication costs), as well as system hardware (i.e., network speed).

OITMAX was historically put in the code to set the maximum allowable number of outer iterations in CTF; however, at some point in CTF's development history, the outer iteration loop has been disabled. CTF will currently take only one outer iteration per timestep. The assumption is that the timestep size will be sufficiently small to ensure that the solution obtained at the end of the single iteration is accurate. OITMAX currently has no effect whatsoever on the CTF simulation, and so it may be set to any value.

IITMAX will have different effects depending on the pressure matrix solver employed. If using the multisimulation-group Gauss-Siedel solver (ISOL $=0$ and NSIM $>1$ ), IITMAX will set the maximum allowable number of iterations of the Gauss-Siedel solution. If one of the PETSc solvers is used (ISOL $=5$ or 7 ), this term serves as the maximum allowable iterations in a single pressure matrix solve. Similar to the EPSO term, it is permissible to set this to a value that reduces the total number of pressure matrix iterations for steady-state solves, while transient solves should converge the pressure matrix more tightly at each timestep. The PETSc default of 1E4 is suggested for transients. Again, the optimal value for IITMAX will depend on problem size, parallel domain decomposition, model conditions, and machine performance, so a one-size-fits-all optimal value cannot be specified; however, limited testing has revealed that a value of 40 seems to work well for single phase models ranging in size from 1 to $2517 \times 17$ assemblies ( 1 to 25 solution domains).

\begin{tabular}{|l|l|l|}
\hline INPUT.3 & EPSO & {$[-]$} \\
\hline Relative tolerance for PETSc pressure matrix solve. The default of $1 \cdot 10^{-5}$ should be used for transients. \\
A larger value may be used for steady-state solutions. \\
\hline Float & Required \\
\hline$>0.0$ &
\end{tabular}

\begin{tabular}{|l|l|l|}
\hline INPUT.3 & OITMAX & {$[-]$} \\
\hline Maximum number of outer iterations - no effect on solution, enter any value \\
\hline Integer & Required \\
\hline$>0$
\end{tabular}




\begin{tabular}{|c|c|c|c|}
\hline INPUT.3 & IITMAX & {$[-]$} & {$[-]$} \\
\hline \multicolumn{4}{|c|}{$\begin{array}{l}\text { If using Gauss-Siedel solver, this is the maximum number of Gauss-Siedel iterations. If using the PETSc } \\
\text { solver, this is the maximum number of PETSc pressure matrix iterations. The suggested value is } 40 \\
\text { for the Gauss-Siedel solver. When using PETSc, the default of } 1 \mathrm{E} 4 \text { should be used for transients. A } \\
\text { smaller value may be used for steady-state solutions. }\end{array}$} \\
\hline Integer & Requirec & & \\
\hline \multicolumn{4}{|l|}{$>0$} \\
\hline
\end{tabular}

\begin{tabular}{|c|c|c|c|}
\hline INPUT.3 & COURANT & {$[-]$} & {$[-]$} \\
\hline \multicolumn{4}{|c|}{$\begin{array}{l}\text { The Courant number to be used in setting the timestep size. If left blank, it will default to the traditional } \\
\text { CTF value of } 0.8 \text {. A suggested value for steady-state simulations is } 3.0 \text {, but check the 'deck.run' file for } \\
\text { numerical stability issues associated with the timestep becoming too large. The proper Courant value } \\
\text { will be highly dependent on the particular case being modeled. Timestep size may also be limited using } \\
\text { controls in Card Group } 15 \text {. }\end{array}$} \\
\hline Float & Optional & & \\
\hline
\end{tabular}

\subsection{Title Card}

\begin{tabular}{|c|c|c|c|}
\hline COBRA.1 & TEXT & {$[-]$} & {$[-]$} \\
\hline \multicolumn{4}{|c|}{ Alphanumeric information to identify the simulation (maximum 30 characters) } \\
\hline Character & Optio & & \\
\hline
\end{tabular}




\section{Card Group 1}

This card group is read by subroutine READ_CARD_1.

\subsection{Selection of Physical Models}

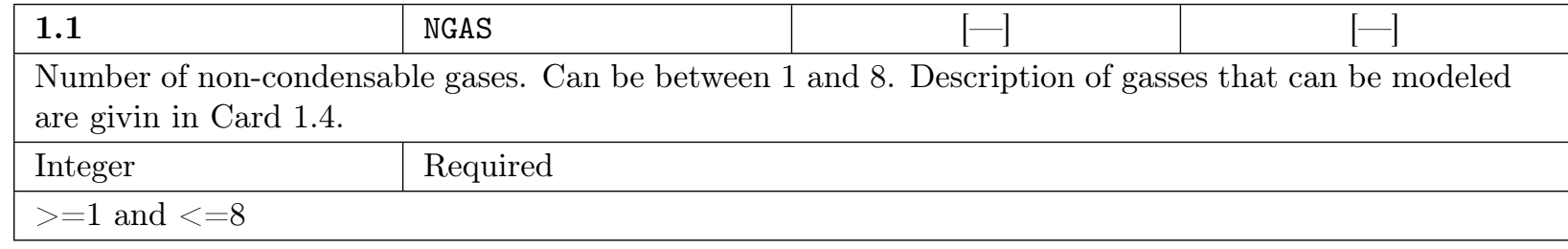




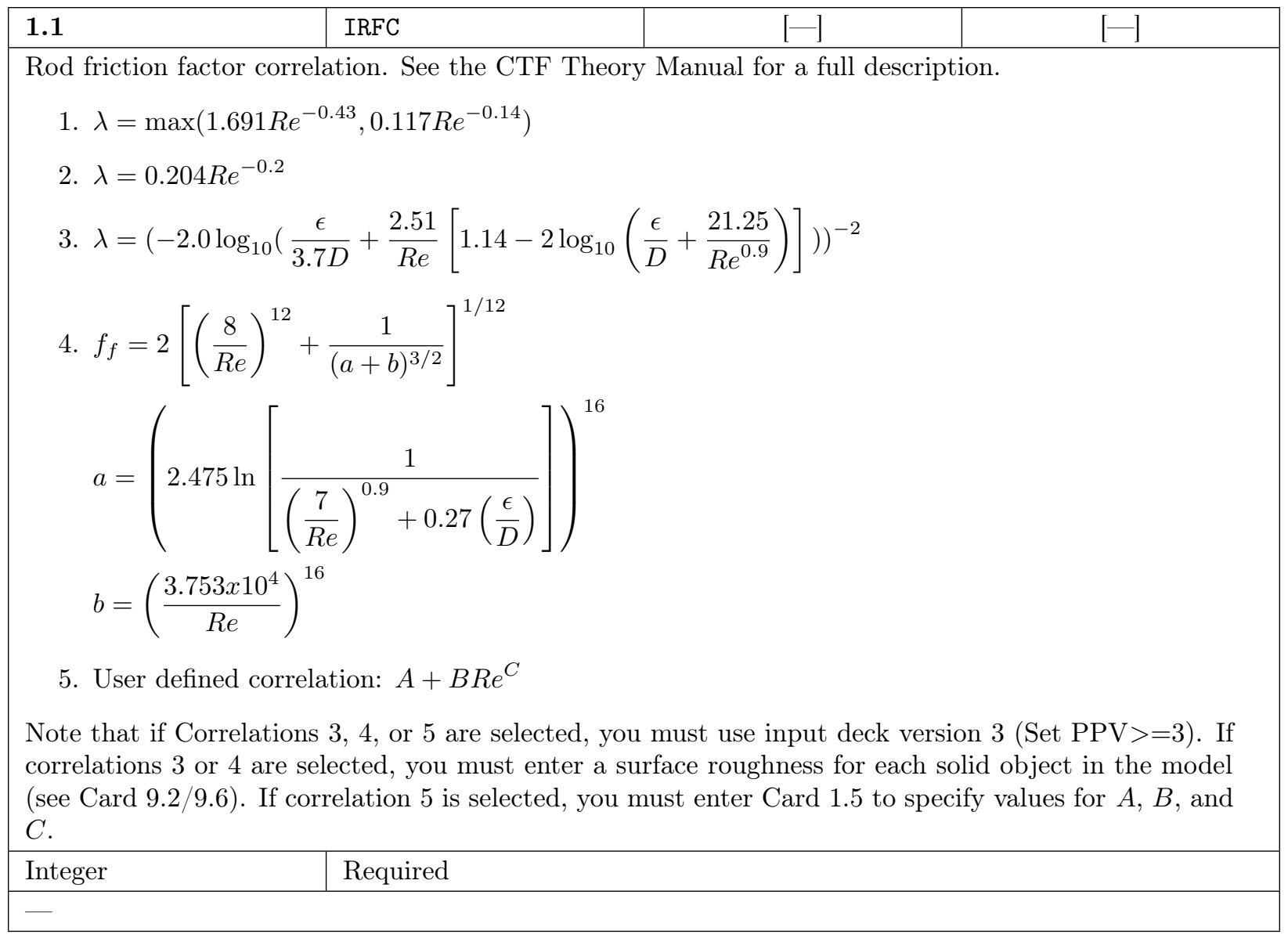

\begin{tabular}{|c|c|c|c|}
\hline 1.1 & EDMOD & {$[-]$} & {$[-]$} \\
\hline \multicolumn{4}{|c|}{ Entrainment and deposition model: } \\
\hline \multicolumn{4}{|c|}{0 - No entrainment or deposition } \\
\hline \multicolumn{4}{|c|}{1 - Original model, which models entrainment and deposition } \\
\hline Integer & Required & & \\
\hline- & & & \\
\hline
\end{tabular}




\begin{tabular}{|c|c|c|c|}
\hline 1.1 & IMIX & {$[-]$} & {$[-]$} \\
\hline \multicolumn{4}{|c|}{ Mixing and void drift model: } \\
\hline \multicolumn{4}{|c|}{0 - Neither mixing nor void drift } \\
\hline \multicolumn{4}{|c|}{1 - User-specified constant two-phase turbulent mixing coefficient } \\
\hline \multicolumn{4}{|c|}{2 - Single-phase mixing coefficient according to Rogers and Rosehart (1972) } \\
\hline \multicolumn{4}{|c|}{$\begin{array}{l}3 \text { - User-specified constant single-phase turbulent mixing coefficient; two-phase multiplier according } \\
\text { to Beus (1970) }\end{array}$} \\
\hline Integer & Required & & \\
\hline - & & & \\
\hline
\end{tabular}

\begin{tabular}{|c|c|c|c|}
\hline 1.1 & ISOL & {$[-]$} & {$[1$} \\
\hline \multicolumn{4}{|c|}{ Solver for the pressure equation: } \\
\hline \multicolumn{4}{|c|}{$\begin{array}{l}0 \text { - Direct Gaussian elimination or Iterative Gauss-Seidel solver } \\
\quad \text { (For pressure equation, selection by means of parameter NSIM in Card Group 4.1) }\end{array}$} \\
\hline \multicolumn{4}{|c|}{3 - Iterative Krylov solver: BCGS (Bi-Conjugate Gradient Method Stabilized) } \\
\hline \multicolumn{4}{|c|}{$\begin{array}{l}5 \text { - Parallel iterative solver using PETSc. Required for parallel runs (CTF will automatically choose } \\
\text { if it is a parallel run) }\end{array}$} \\
\hline \multicolumn{4}{|c|}{$\begin{array}{l}\text { * - NOTE: Solution options } 1,2 \text {, and } 4 \text { were removed as there were memory issues with option } 1 . \\
\text { Options } 2 \text { and } 4 \text { were not tested }\end{array}$} \\
\hline Integer & Required & & \\
\hline - & & & \\
\hline
\end{tabular}

\begin{tabular}{|c|c|c|c|}
\hline 1.1 & GINIT & {$[\mathrm{kg} / \mathrm{s}]$} & {$[\mathrm{lbm} / \mathrm{s}]$} \\
\hline \multicolumn{4}{|c|}{$\begin{array}{l}\text { Value of the mass flow rate to initialize mass flow rate in the entire CTF mesh. The cross sectional } \\
\text { area used to calculate the mass flux will be the Section } 1 \text { inlet cross sectional area, as is done for GTOT. } \\
\text { Enter } 0.0 \text { if specifying the mass flux for initialization. }\end{array}$} \\
\hline Float & Require & & \\
\hline
\end{tabular}




\begin{tabular}{|l|l|c|}
\hline $\mathbf{1 . 1}$ & NOTRANS & {$[-]$} \\
\hline Flag to specify whether CTF should use TEND from Card Group 15 to end the simulation or if conver- \\
gence criteria are used instead: \\
0 - Use Card Group 15 transient information \\
- Use internal code convergence criteria \\
- Same as option 0, except that VTK files will be printed at each EDINT interval of the transient. \\
If selected, the files will be named according to the "filename.rods.vtk.N" convention, where \\
"N" is the number of the file. \\
\hline Integer \\
\hline-
\end{tabular}

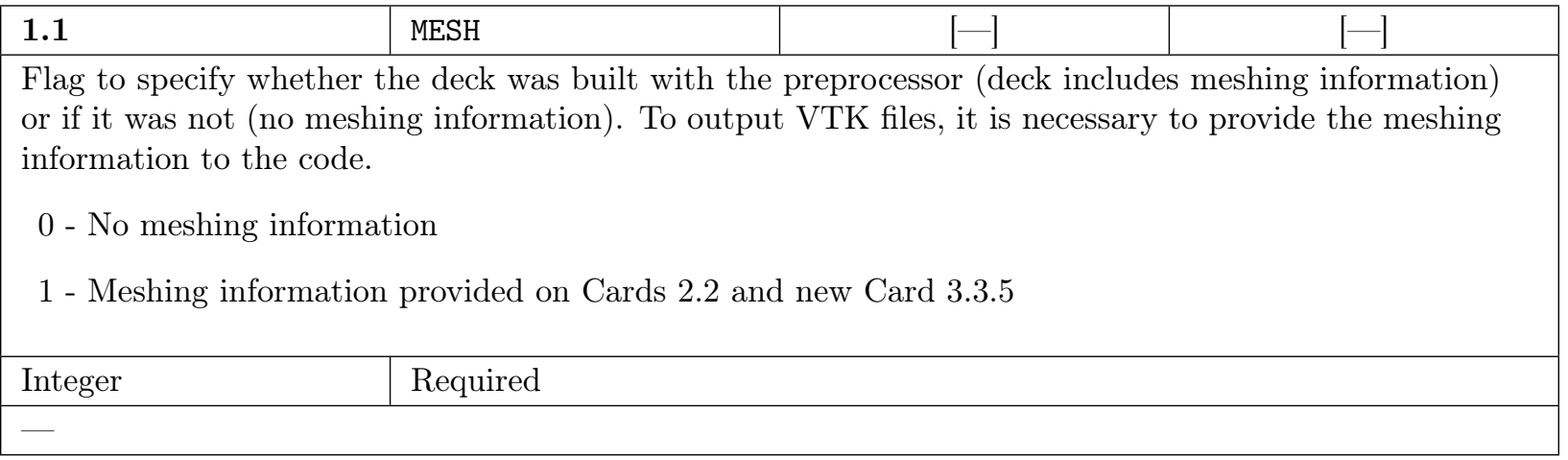

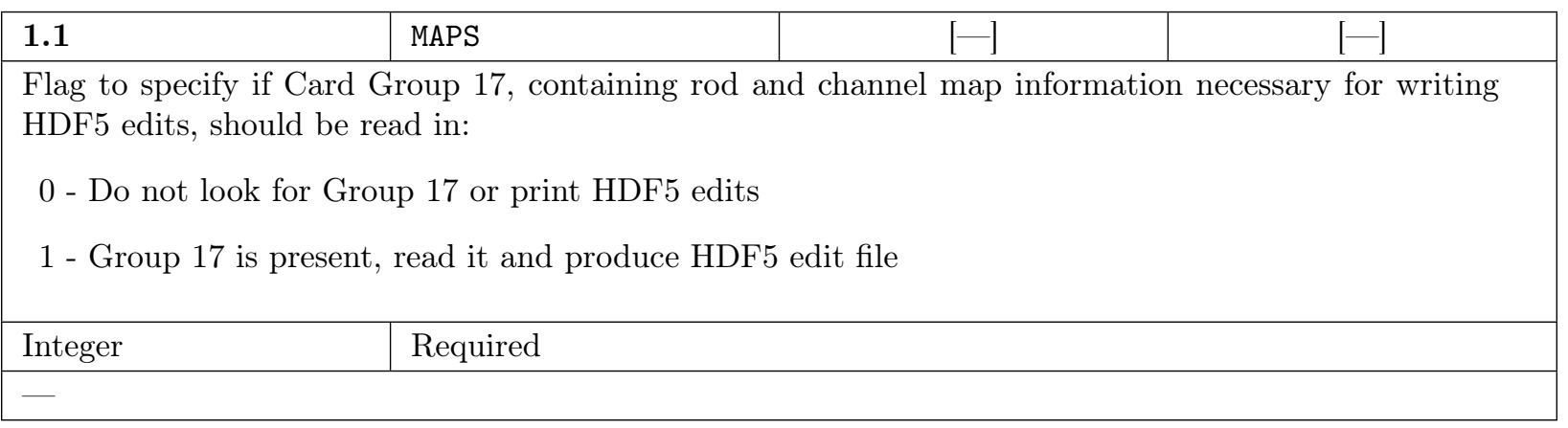




\begin{tabular}{|c|c|c|c|}
\hline 1.1 & IPROPS & {$[-]$} & {$[-]$} \\
\hline \multicolumn{4}{|c|}{ Fluid property tables to use: } \\
\hline \multicolumn{4}{|c|}{0 - Original CTF water property tables (mix of various sources) } \\
\hline \multicolumn{4}{|c|}{1 - IAPWS IF97 water property tables (direct correlation evaluations) } \\
\hline \multicolumn{4}{|c|}{$\begin{array}{l}2 \text { - IAPWS IF97 water property tables, using a second-order central difference approximation for } \\
\text { property derivatives with respect to temperature and pressure }\end{array}$} \\
\hline \multicolumn{4}{|c|}{$\begin{array}{l}3 \text { - IAPWS IF97 water property tables (lookup tables are generated from the direct correlation eval- } \\
\text { uations using Option 1, so this method will have some error but performs much faster) }\end{array}$} \\
\hline \multicolumn{4}{|c|}{11 - FLiBe liquid salt property correlations } \\
\hline Integer & Required & & \\
\hline- & & & \\
\hline
\end{tabular}

\begin{tabular}{|c|c|c|c|}
\hline 1.1 & MFLX & {$[-]$} & {$[-]$} \\
\hline \multicolumn{4}{|c|}{$\begin{array}{l}\text { Option to set the inlet boundary condition and the mass flow initialization using a mass flux rather } \\
\text { than mass flow rate. If using this option, GTOT and GINIT must be set to 0.0. CTF will use the mass } \\
\text { flux supplied to calculate GTOT and GINIT based on the channel flow areas. }\end{array}$} \\
\hline \multicolumn{4}{|c|}{0 - Do not specify mass flow $\mathrm{BC}$ as mass flux } \\
\hline \multicolumn{4}{|c|}{1 - Specify the mass flow BC as mass flux } \\
\hline Integer & Required & & \\
\hline - & & & \\
\hline
\end{tabular}

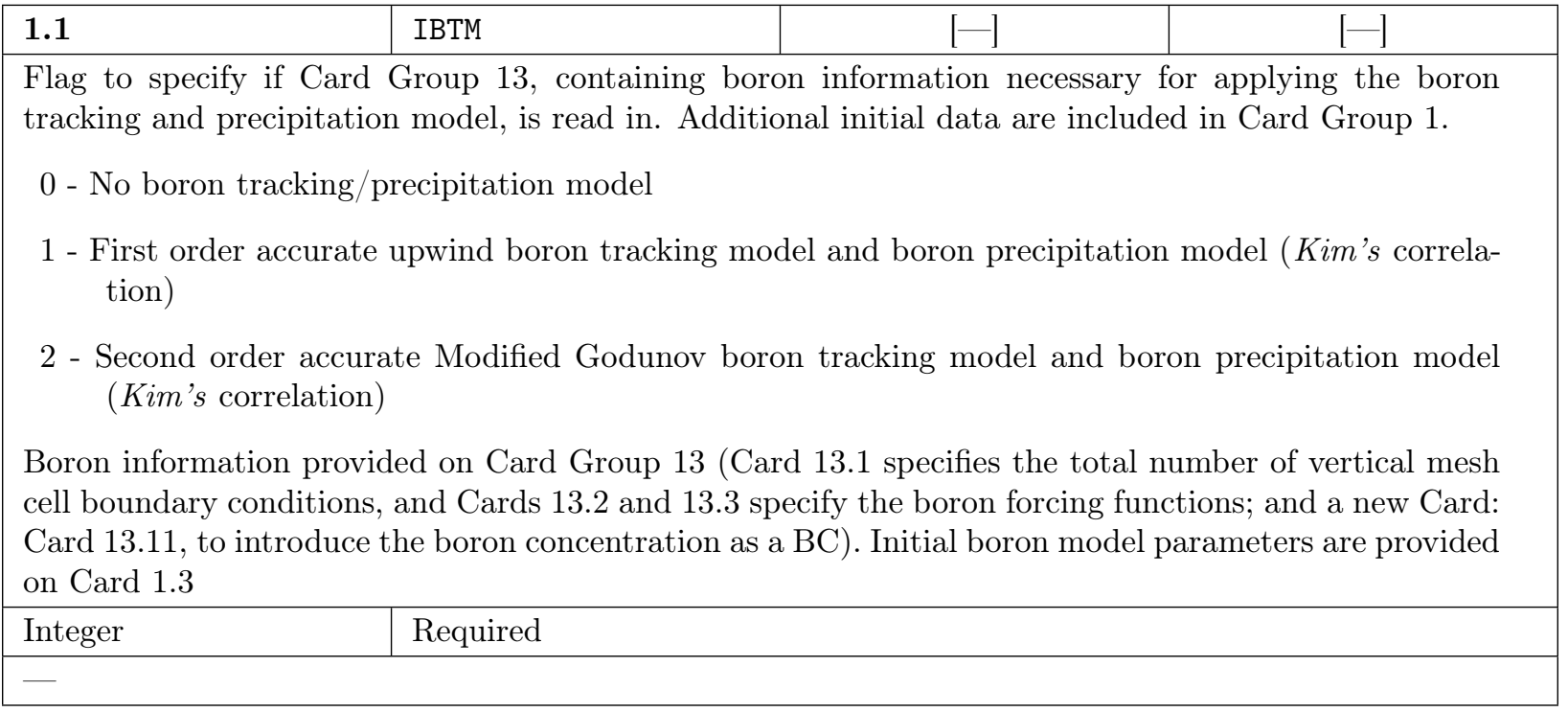




\begin{tabular}{|c|c|c|c|}
\hline 1.1 & PPV & {$[-]$} & {$[-]$} \\
\hline \multicolumn{4}{|c|}{$\begin{array}{l}\text { The input deck version number. See Table } 1 \text { for a meaning of the version numbers. Always try to use } \\
\text { the most recent version of the input deck. }\end{array}$} \\
\hline Integer & Required & & \\
\hline- & & & \\
\hline
\end{tabular}

\begin{tabular}{|l|l|l|}
\hline $\mathbf{1 . 1}$ & BWRMODEL & {$[-]$} \\
\hline It is not recommended this option be used when generating a CTF input deck by hand. It is intended \\
that the preprocessor should be used to activate this feature. This feature tells CTF that this is a \\
BWR model where the assemblies are completely separate from one another (not connected at top or \\
bottom). This feature will enable an outer iteration loop in CTF that will adjust the inlet mass flow \\
rates to make the pressure drop in all fuel assemblies equal. Model must be one axial section and \\
have inlet mass flow rate specified in the first level of every channel. Must also be a parallel model. \\
Note that, if this model is enabled, then the pressure matrix solver will be set to ISOL=3 by default. \\
Options: \\
0-Disable outer iteration loop \\
1-Enable outer iteration loop (use for multi-assembly BWR models created by preprocessor) \\
\hline Integer \\
\hline - Required \\
\hline
\end{tabular}

\subsection{Global Boundary Conditions}

\begin{tabular}{|c|c|c|c|}
\hline 1.2 & GTOT & {$[\mathrm{kg} / \mathrm{s}]$} & {$[\mathrm{lbm} / \mathrm{s}]$} \\
\hline \multicolumn{4}{|c|}{$\begin{array}{l}\text { Total inlet mass flow rate. This is a convenience feature so that the user does not need to enter the } \\
\text { inlet mass flow rate for every channel in the model in Card } 13 \text {. If this is non-zero, then the mass flow } \\
\text { rate and enthalpy boundary conditions entered on Card } 13 \text { should all be zero for the inlet plane. The } \\
\text { flow areas of those channels will be used to calculate the inlet mass flow rate of each channel. If any } \\
\text { inlet mass flow rate plus enthalpy boundary condition entered on Card } 13 \text { is non-zero, then this input } \\
\text { (GTOT) will be ignored, and the explicit values entered on Card } 13 \text { will be used instead. Enter } 0.0 \text { if } \\
\text { setting MFLX = } 1 \text {. }\end{array}$} \\
\hline Float & Required & & \\
\hline \multicolumn{4}{|l|}{ Any } \\
\hline 1.2 & AFLUX & {$[\mathrm{kW} / \mathrm{m}]$} & {$[\mathrm{kW} / \mathrm{ft}]$} \\
\hline \multicolumn{4}{|c|}{$\begin{array}{l}\text { Average linear heat rate per rod. To calculate AFLUX the total bundle power is divided by the total rod } \\
\text { length multiplied by the total number of rods. }\end{array}$} \\
\hline Float & Required & & \\
\hline$>0.0$ & & & \\
\hline
\end{tabular}




\begin{tabular}{|l|l|l|}
\hline $\mathbf{1 . 2}$ & DHFRAC & {$[-]$} \\
\hline $\begin{array}{l}\text { Fraction of local heat rate generated by the heater rods which is released directly into the coolant. (As } \\
\text { a coarse approach, the direct heat is added to the liquid only - not to the vapor). }\end{array}$ \\
\hline Float & Required \\
\hline$>=0.0$ and $<=1.0$ & \\
\hline
\end{tabular}

\begin{tabular}{|c|c|c|c|}
\hline 1.2 & MFLUX & {$\left[\mathrm{kg} / \mathrm{s}-\mathrm{m}^{2}\right]$} & {$\left[\mathrm{lbm} / \mathrm{s}-\mathrm{ft}^{2}\right]$} \\
\hline \multicolumn{4}{|c|}{$\begin{array}{l}\text { The inlet and initialization mass flux. Only read if MFLX }=1 \text {. If inlet mass flow rate plus enthalpy } \\
\text { boundary conditions in Card } 13 \text { are all set to } 0.0 \text { mass flow rate, then this value will be used to calculate } \\
\text { their individual inlet mass flow rates. If any inlet boundary condition entered on Card } 13 \text { is nonzero, } \\
\text { then this parameter will be ignored in terms of calculating the inlet mass flow rate. However, it will } \\
\text { still be used for calculation of the initial mass flow rate distribution in the model. }\end{array}$} \\
\hline Float & Conditional & & \\
\hline Any & & & \\
\hline
\end{tabular}

\subsection{Initial Conditions}

\begin{tabular}{|l|l|c|c|}
\hline $\mathbf{1 . 3}$ & PREF & {$[\mathrm{bar}]$} & {$[\mathrm{psi}]$} \\
\hline Initial pressure in the fluid domain & & \\
\hline Float & Required & \\
\hline$>0.0$ & \\
\hline
\end{tabular}

\begin{tabular}{|c|c|c|c|}
\hline 1.3 & HIN & {$[\mathrm{kJ} / \mathrm{kg}]$} & {$[\mathrm{BTU} / \mathrm{lbm}]$} \\
\hline \multicolumn{4}{|c|}{$\begin{array}{l}\text { Initial enthalpy in the fluid domain } \\
* * \text { Note: Either HIN or TIN must be supplied** } \\
\text { An enthalpy value is entered as a positive value, such as } 1300 \mathrm{~kJ} / \mathrm{kg} \text {. }\end{array}$} \\
\hline Float & Req & & \\
\hline$>0.0$ & & & \\
\hline
\end{tabular}

\begin{tabular}{|c|c|c|c|}
\hline 1.3 & TIN & {$\left[{ }^{\circ} \mathrm{C}\right]$} & {$\left[{ }^{\circ} \mathrm{F}\right]$} \\
\hline \multicolumn{4}{|c|}{$\begin{array}{l}\text { Initial temperature in the fluid domain } \\
* * \text { Note: Either HIN or TIN must be supplied }{ }^{* *} \\
\text { A temperature value is entered by setting it negative. For example, } 310{ }^{\circ} \mathrm{C} \text { would be entered as }-310.0 \\
\text { for TIN. }\end{array}$} \\
\hline Float & Requ & & \\
\hline$<0.0$ & & & \\
\hline
\end{tabular}




\begin{tabular}{|l|l|l|l|}
\hline $\mathbf{1 . 3}$ & HGIN & {$[\mathrm{kJ} / \mathrm{kg}]$} & {$[\mathrm{BTU} / \mathrm{lbm}]$} \\
\hline Enthalpy of non-condensable gas mixture & \\
\hline Float & Required & \\
\hline$>0.0$ & \\
\hline
\end{tabular}

\begin{tabular}{|l|l|l|l|}
\hline $\mathbf{1 . 3}$ & VFRAC (1) & {$[-]$} & {$[-]$} \\
\hline Initial liquid volume fraction in the liquid-vapor-gas mixture & \\
\hline Float & Required \\
\hline$>=0.0$ and $<=1.0$ & \\
\hline
\end{tabular}

\begin{tabular}{|l|l|l|l|}
\hline $\mathbf{1 . 3}$ & VFRAC (2) & {$[-]$} & {$[-]$} \\
\hline Initial vapor volume fraction in the vapor-gas mixture & & \\
\hline Float & Required & \\
\hline$>=0.0$ and $<=1.0$ & \\
\hline
\end{tabular}

\begin{tabular}{|c|c|c|c|}
\hline 1.3 & BRIN & [ppm] & [ppm] \\
\hline \multicolumn{4}{|c|}{ Initial boron concentration (uniform distribution) } \\
\hline Float & Conditional & & \\
\hline$>=0.0$ & & & \\
\hline
\end{tabular}

\begin{tabular}{|c|c|c|c|}
\hline 1.3 & RDIF & {$[-]$} & {$[-]$} \\
\hline \multicolumn{4}{|c|}{ Boron physical diffusion coefficient: } \\
\hline \multicolumn{4}{|c|}{0.0 - Suggested value (first order upwind scheme, IBTM $=1$ ) } \\
\hline \multicolumn{4}{|c|}{1.0 - Suggested value (modified Godunov scheme, IBTM $=2$ ) } \\
\hline Float & Cond & & \\
\hline - & & & \\
\hline
\end{tabular}

\begin{tabular}{|l|l|l|l|}
\hline $\mathbf{1 . 4}$ & GTYPE(I) & {$[-]$} & {$[-]$} \\
\hline $\begin{array}{l}\text { Name of non-condensible gas; acceptable names: } \\
\text { air, argo, heli, hydr, kryp, nitr, oxyg, xeno }\end{array}$ & \\
\hline Character & Required \\
\hline-
\end{tabular}

\begin{tabular}{|l|l|c|c|}
\hline $\mathbf{1 . 4}$ & VFRAC $(I+2)$ & {$[-]$} & {$[-]$} \\
\hline Initial volume fraction of non-condensable gas $I$ in the vapor- gas mixture & \\
\hline Float & Required \\
\hline$>=0.0$ and $<=1.0$ & \\
\hline
\end{tabular}




\subsection{Custom Friction Model Input}

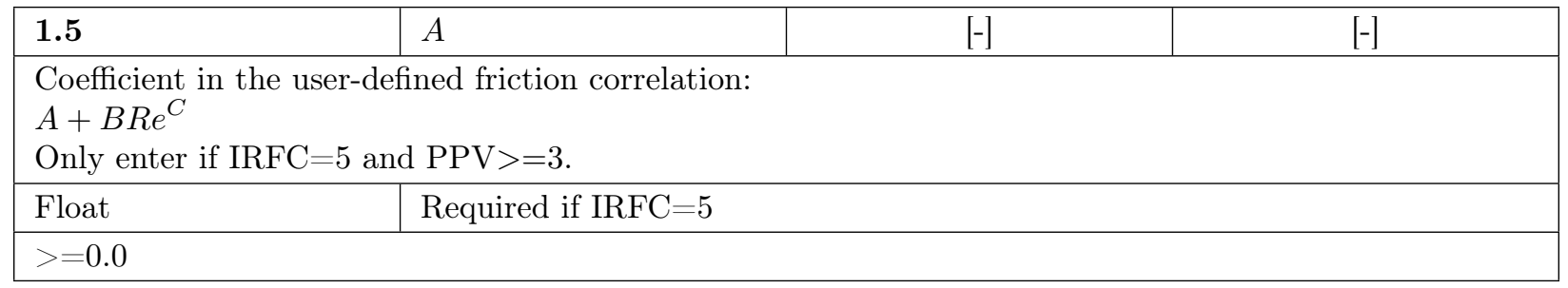

\begin{tabular}{|c|c|c|c|}
\hline 1.5 & $B$ & {$[-]$} & {$[-]$} \\
\hline \multicolumn{4}{|c|}{$\begin{array}{l}\text { Coefficient in the user-defined friction correlation: } \\
A+B R e^{C} \\
\text { Only enter if } \mathrm{IRFC}=5 \text { and } \mathrm{PPV}>=3 \text {. }\end{array}$} \\
\hline Float & Required if $\mathrm{IRFC}=5$ & & \\
\hline$>=0.0$ & & & \\
\hline
\end{tabular}

\begin{tabular}{l|l|c|c|}
\hline $\mathbf{1 . 5}$ & $C$ & {$[-]$} & {$[-]$} \\
\hline Coefficient in the user-defined friction correlation: & & \\
$A+B R e^{C}$ & \\
Only enter if IRFC $=5$ and $\mathrm{PPV}>=3$. & \\
\hline Float & Required if IRFC $=5$ & \\
\hline$>=0.0$ & \\
\hline
\end{tabular}

\subsection{Under-relaxation coefficients}

Several important time under-relaxation coefficients used in CTF have been exposed to the user as optional $\mathrm{key} /$ value pairs. If you omit the following lines, then all under-relaxation coefficients will be set to their defaults. It is not recommended that these coefficients be used unless there is an issue with converging the code and other options for stabilizing the solution have failed (e.g., turning on the conduction solve and adjusting RTWFP). It is not recommended these coefficients be used at all for transients, as they will impact the transient solution.

The under-relaxation coefficients are used to perform logarithmic interpolation.

$$
x^{n}=\left(x^{n}\right)^{\beta} *\left(x^{n-1}\right)^{1-\beta}
$$

The under-relaxation coefficient must be entered as a key/value pair. The key is the name of the coefficient surrounded by curly braces. The value is the coefficient, which shall be between 0.0 and 1.0. The key and value shall be separated by whitespace. 


\begin{tabular}{|c|c|c|c|}
\hline 1.6 & beta_entr & {$[-]$} & {$[-]$} \\
\hline \multicolumn{4}{|c|}{ Under-relaxation coefficient for the droplet entrainment rate. Default 0.3 . } \\
\hline Float & Optional & & \\
\hline$>0.0$ & & & \\
\hline
\end{tabular}

\begin{tabular}{|l|l|c|c|}
\hline $\mathbf{1 . 6}$ & beta_deentr & {$[-]$} & {$[-]$} \\
\hline \multicolumn{2}{|l|}{ Under-relaxation coefficient for the droplet de-entrainment rate. Default 0.1.} \\
\hline Float & Optional \\
\hline$>0.0$ & \\
\hline
\end{tabular}

\begin{tabular}{|l|l|l|l|}
\hline $\mathbf{1 . 6}$ & beta_inthtc & {$[-]$} & {$[-]$} \\
\hline Under-relaxation coefficient for the interfacial heat transfer coefficient. Default 0.1. \\
\hline Float & Optional \\
\hline$>0.0$ & \\
\hline
\end{tabular}

\begin{tabular}{|c|c|c|c|}
\hline 1.6 & beta_int_vl & {$[-]$} & {$[-]$} \\
\hline \multicolumn{4}{|c|}{ Under-relaxation coefficient for the interfacial vapor/liquid drag. Default 0.3 . } \\
\hline Float & Optional & & \\
\hline$>0.0$ & & & \\
\hline
\end{tabular}

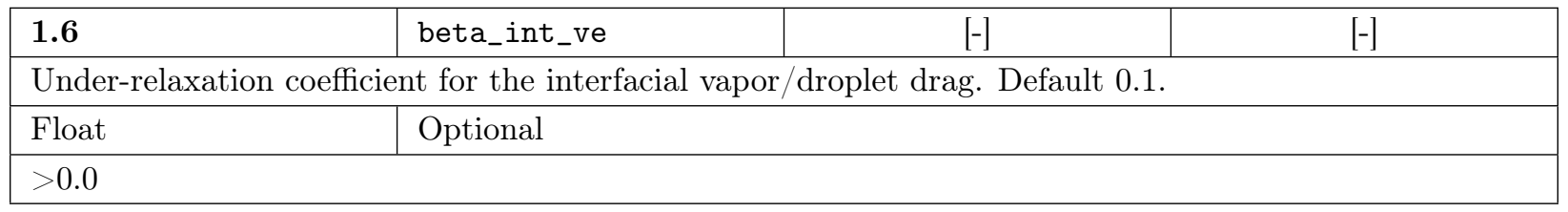

\begin{tabular}{|l|l|l|l|}
\hline $\mathbf{1 . 6}$ & beta_int_vl_gap & {$[-]$} & {$[-]$} \\
\hline Under-relaxation coefficient for the interfacial vapor/liquid drag in gaps. Default 0.1. & \\
\hline Float & Optional \\
\hline$>0.0$ & \\
\hline
\end{tabular}

\begin{tabular}{|l|l|c|c|}
\hline 1.6 & beta_int_ve_gap & {$[-]$} & {$[-]$} \\
\hline \multicolumn{2}{|l|}{ Under-relaxation coefficient for the interfacial vapor/droplet drag in gaps. Default 0.1. } \\
\hline Float & Optional \\
\hline$>0.0$ &
\end{tabular}

\begin{tabular}{|l|l|c|c|}
\hline $\mathbf{1 . 6}$ & beta_htc & {$[-]$} & {$[-]$} \\
\hline \multicolumn{2}{|l|}{ Under-relaxation coefficient for the solid/fluid heat transfer coefficient. Default 0.2.} \\
\hline Float & Optional \\
\hline$>0.0$ & \\
\hline
\end{tabular}




\begin{tabular}{|l|l|l|l|}
\hline $\mathbf{1 . 6}$ & use_lane_model & {$[-]$} & {$[-]$} \\
\hline $\begin{array}{l}\text { Specify to select the annular mist model. Set to .true. to use the Lane model for annular mist flow } \\
\text { regime closure models or .false. to use the traditional models. Defaults to traditional. }\end{array}$ \\
\hline Bool & Optional \\
\hline-
\end{tabular}

\subsection{CTF-Residual (CTF-R)}

This novel capability of CTF is based on the use of residual equations to solve single-phase problems and is therefore called CTF-R. CTF-R does not have many of the computational simplifications, mathematical assumptions, or inherent conservatism that are part of the legacy code. The elimination of these assumptions will have a drastic impact on the flexibility, accuracy, and optimization of the code, all of which have important benefits. The current version of CTF-R is capable of modeling heated single-phase flows in small models. The development is backed by a robust set of SQA activities that provide confidence in the code.

\begin{tabular}{|l|l|c|c|}
\hline $\mathbf{1 . 7}$ & residual_solver & {$[-]$} & {$[-]$} \\
\hline $\begin{array}{l}\text { Specify to select the residual formulation. Set to .true. to use the residual formulation of the conser- } \\
\text { vation equations or .false. to use the traditional formulation. Defaults to traditional. }\end{array}$ \\
\hline Bool & Optional \\
\hline-
\end{tabular}

\subsection{Onset of nucleate boiling (ONB)}

This is a switch to turn on the onset of the nucleate boiling model as described in the theory manual. The current default wall boiling model is set to turn on as soon as the wall temperature exceeds the local fluid saturation temperature. The development is backed by a regression test that provides confidence in the ONB model.

\begin{tabular}{|c|c|c|c|}
\hline 1.8 & use_onb_model & {$[-]$} & {$[-]$} \\
\hline \multicolumn{4}{|c|}{$\begin{array}{l}\text { Specify to select the onset of nucleate boiling model. Set to .true. to use the ONB model for wall } \\
\text { boiling or .false. to use the traditional model without an ONB model. Defaults to no ONB model. The } \\
\text { current default wall boiling model (ONB off) is set to turn on as soon as the wall temperature exceeds } \\
\text { the local fluid saturation temperature. This turns the boiling model on after the ONB temperature } \\
\text { defined by the model of Basu is reached, as described in the Theory Manual. }\end{array}$} \\
\hline Bool & Optional & & \\
\hline - & & & \\
\hline
\end{tabular}




\subsection{Notes on Card Group 1}

The initial mass flow rate is set to 0.0 by default. Therefore, a time-dependent âĂIJrampâĂİ for the inlet mass flow rate should be applied (see description in CARD GROUP 13). Variables PREF and HIN are used to determine the initial properties of the fluid in computational domain. Because the mass conservation equation for non-condensable gases is not actively solved in this version of CTF, the volume fraction of vapor in the vapor-gas mixture should not be less than 0.9999! Variables BRIN and RDIF are only read if the boron tracking/precipitation model is activated (IBTM $\neq 0)$. RDIF is only used when IBTM $=2$. 


\section{Card Group 2}

This card group is read by subroutine READ_CARD_2.

The first line indicates the group number: NGROUP $=2$

\begin{tabular}{|l|l|l|l|}
\hline $\mathbf{2 . 1}$ & NCHANL & {$[-]$} & {$[-]$} \\
\hline \multicolumn{2}{|l|}{ Total number of subchannels } & \\
\hline Integer & Required & \\
\hline$>0$ &
\end{tabular}

\begin{tabular}{l|l|l|l|}
\hline $\mathbf{2 . 1}$ & NDUM2: NDUM14 & {$[-]$} & {$[-]$} \\
\hline $\begin{array}{l}\text { Not used, but entry is obligatory: } \\
0 \text { - Suggested value }\end{array}$ \\
\begin{tabular}{ll} 
Integer & Required \\
\hline 0 &
\end{tabular} \\
\hline
\end{tabular}

Cards 2.2 and 2.3 are read in pairs NCHANL times.

CARD 2.2: I, $\operatorname{AN}(I), P W(I), \operatorname{ABOT}(I), \operatorname{ATOP}(I), \operatorname{NAMGAP}(I), X(I), Y(I), \operatorname{XSIZ}(I), \operatorname{YSIZ}(I)$

\begin{tabular}{|l|l|c|c|}
\hline $\mathbf{2 . 2}$ & I & {$[-]$} & {$[-]$} \\
\hline $\begin{array}{l}\text { Index of subchannel. Each channel index must be unique and entered in ascending order with no } \\
\text { skipped channel indices. }\end{array}$ & Required \\
\hline Integer &
\end{tabular}

\begin{tabular}{|l|l|c|c|}
\hline $\mathbf{2 . 2}$ & AN (I) & {$\left[\mathrm{m}^{2}\right]$} & {$\left[\mathrm{in}^{2}\right]$} \\
\hline Nominal channel area & & \\
\hline Float & Required & \\
\hline$>0.0$ & \\
\hline
\end{tabular}




\begin{tabular}{|c|c|c|c|}
\hline 2.2 & $\mathrm{PW}(\mathrm{I})$ & {$[\mathrm{m}]$} & [in] \\
\hline \multicolumn{4}{|c|}{ Wetted perimeter } \\
\hline Float & Required & & \\
\hline$>0.0$ & & & \\
\hline
\end{tabular}

\begin{tabular}{|c|c|c|c|}
\hline 2.2 & $\mathrm{ABOT}(\mathrm{I})$ & {$\left[\mathrm{m}^{2}\right]$} & {$\left[\mathrm{in}^{2}\right]$} \\
\hline \multicolumn{4}{|c|}{$\begin{array}{l}\text { Area of the bottom of the channel for use in the momentum equation. If ABOT(I) is entered as zero, } \\
\text { it is set to } \mathrm{AN}(\mathrm{I}) \text {. }\end{array}$} \\
\hline Float & Required & & \\
\hline$>=0.0$ & & & \\
\hline
\end{tabular}

\begin{tabular}{|c|c|c|c|}
\hline 2.2 & $\operatorname{ATOP}(I)$ & {$\left[\mathrm{m}^{2}\right]$} & {$\left[\mathrm{in}^{2}\right]$} \\
\hline \multicolumn{4}{|c|}{$\begin{array}{l}\text { Area of the top of the channel for use in the momentum equation. If ATOP(I) is entered as zero, it is } \\
\text { set to AN (I). }\end{array}$} \\
\hline Float & Required & & \\
\hline$>=0.0$ & & & \\
\hline
\end{tabular}

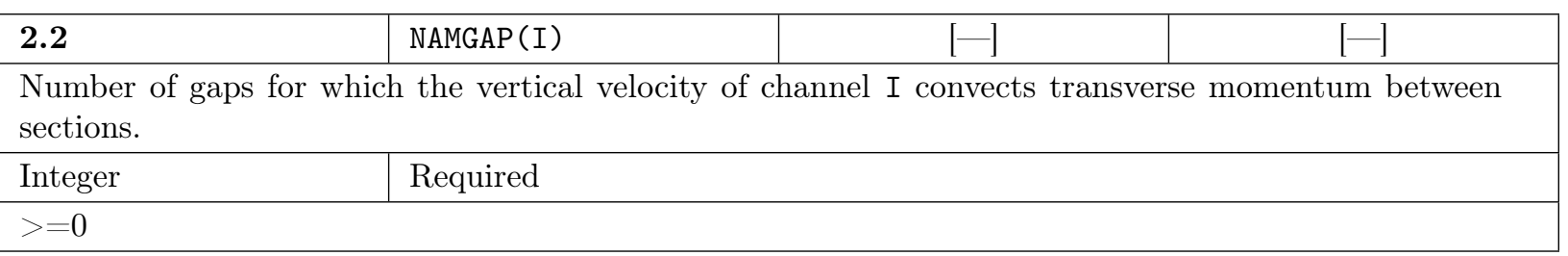

The following data are only appended to CARD GROUP 2.2 if MESH $=1$ (see Card 1.1).

\begin{tabular}{|c|c|c|c|}
\hline 2.2 & $\mathrm{X}(\mathrm{I})$ & {$[\mathrm{m}]$} & [in] \\
\hline \multicolumn{4}{|c|}{ The X location of the geometric center of channel I } \\
\hline Float & Cond & & \\
\hline Any & & & \\
\hline
\end{tabular}

\begin{tabular}{|c|c|c|c|}
\hline 2.2 & $\mathrm{Y}(\mathrm{I})$ & {$[\mathrm{m}]$} & [in] \\
\hline \multicolumn{4}{|c|}{ The Y location of the geometric center of channel I } \\
\hline Float & \multicolumn{3}{|c|}{ Conditional - if MESH $=1$} \\
\hline Any & & & \\
\hline
\end{tabular}

\begin{tabular}{|c|c|c|c|}
\hline 2.2 & $\operatorname{XSIZ}(\mathrm{I})$ & [m] & [in] \\
\hline \multicolumn{4}{|c|}{ The size of channel I in the $\mathrm{X}$ direction } \\
\hline Float & \multicolumn{3}{|c|}{ Conditional - if MESH $=1$} \\
\hline$>0.0$ & & & \\
\hline
\end{tabular}




\begin{tabular}{|l|l|c|c|}
\hline $\mathbf{2 . 2}$ & YSIZ(I) & {$[\mathrm{m}]$} & [in] \\
\hline \multicolumn{2}{|l|}{ The size of channel I in the Y direction } & \\
\hline Float & Conditional - if MESH $=1$ \\
\hline$>0.0$ & \\
\hline
\end{tabular}

Note 1: Do not enter PW(I) equal to zero, because in that case the calculated hydraulic diameter of the channel becomes infinite.

Note 2: In case of more than one axial sections, NAMGAP(I) has to be specified differently from zero and CARD 2.3 has to be specified, too, in order to get a correct momentum transfer in the last axial cell of each section. In case of only one axial section, the input values of CARD 2.3 are ignored. They are created automatically by the code. That means, NAMGAP(I) can be set to zero and CARD 2.3 can be omitted. (The procedure was not yet tested for reverse flow conditions!)

Note 3: For the meshing information, the $\mathrm{X}$ and $\mathrm{Y}$ positions should specify the geometric center of the channel (the channel is assumed rectangular in shape). The XSIZ and YSIZ terms should create a box that completely covers the bounds of the channel. The X and Y positions should NOT be the flow area centroid. The selected origin of the model is up to the user. The $\mathrm{X}$ and $\mathrm{Y}$ terms can be positive or negative. These terms are used for visualizing data in the VTK file and will not impact the code solution. This information may be omitted by setting MESH=0 on Card 1.1. See Figure 4.1 for an example of these terms in a graphical way. A group of 9 subchannels is shown, setup as a single assembly. The zero location is in the lower-left. The four parameters are defined for channel 5. Similarly, an example is given for a quarter core model (see Figure 4.2).

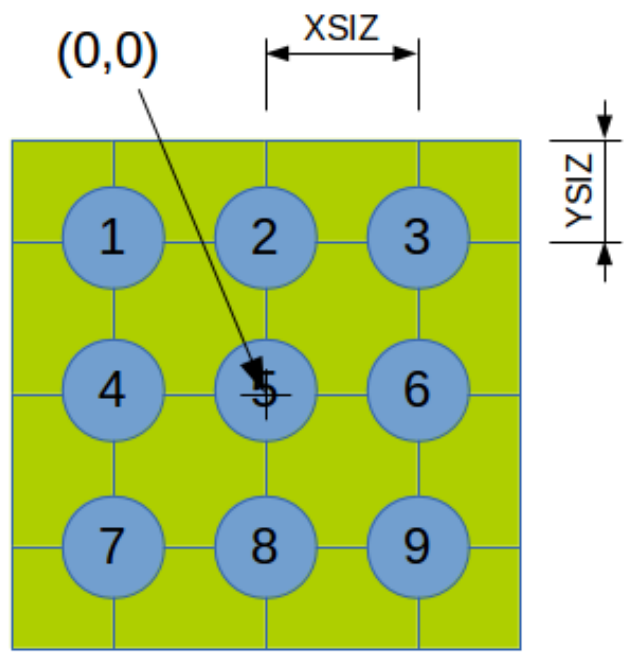

Figure 4.1: Definition of the X, Y, XSIZ, and YSIZ terms for Card 2.2 for a single assembly, plus a suggestion of where the origin can be placed 


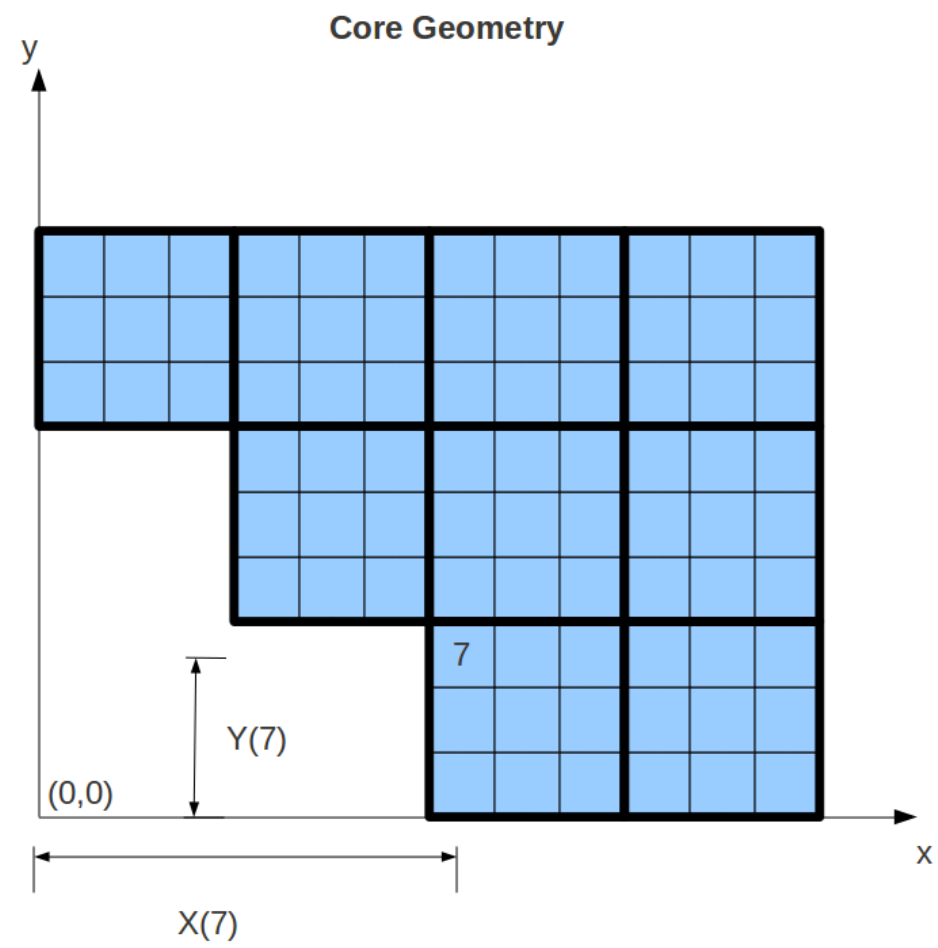

Figure 4.2: Definition of the $(0,0)$ location and $\mathrm{X}$ and $\mathrm{Y}$ for core geometry

$\operatorname{Card} 2.3: \operatorname{INODE}(I, N), \operatorname{KGAPB}(I, N), \operatorname{KGAPA}(I, N) ; N=1: \operatorname{NAMGAP}(I)$

Card 2.3 is only read if NAMGAP(I) $>0$ for channel $\mathrm{I}$.

\begin{tabular}{|c|c|c|c|}
\hline 2.3 & $\operatorname{INODE}(\mathrm{I}, \mathrm{N})$ & {$[-]$} & {$[-]$} \\
\hline \multicolumn{4}{|c|}{$\begin{array}{l}\text { The index number of the node where the vertical velocity of channel I convects transverse momentum } \\
\text { across a section boundary. } \\
\text { Note: INODE will be either at the bottom of the channel (INODE }(I, N)=1) \text {, or the top of the channel, } \\
\text { (INODE }(I, N)=\text { NONODE }+1 \text { ), where NONODE is the number of axial levels in the section containing } \\
\text { channel I. INODE is defined in the section where the vertical momentum equation is solved. }\end{array}$} \\
\hline Integer & Conditional & hann & \\
\hline
\end{tabular}




\begin{tabular}{l}
\hline $\mathbf{2 . 3} \quad \operatorname{KGAPB}(\mathrm{I}, \mathrm{N})$ \\
\hline The index number of the gap below the section boundary: \\
0 - If there is no gap below the section boundary \\
Note: If KGAPB $\neq 0$, the positive velocity of channel $I$ at INODE $(I, N)$ convects transverse momentum \\
from $K G A P B$ into KGAPA. The negative velocity of channel I at INODE $(I, N)$ convects transverse momen- \\
tum from KGAPA into KGAPB. \\
If KGAPB $=0$, then this momentum is dissipated. \\
\hline Integer $\quad$ Conditional - if NAMGAP $(\mathrm{I})>0$ for channel I \\
\hline$>0$ and $<=$ total number of gaps in model
\end{tabular}

\begin{tabular}{l}
\hline $\mathbf{2 . 3} \quad$ KGAPA $(\mathrm{I}, \mathrm{N})$ \\
\hline The index number of the gap above the section boundary: \\
0 - If there is no gap above the section boundary \\
Note: If KGAPB $\neq 0$, the positive velocity of channel I at INODE $(I, N)$ convects transverse momentum \\
from KGAPB (if KGAPB $\neq 0$ ) into KGAPA. The negative velocity of channel I at INODE $(I, N)$ convects \\
transverse momentum from KGAPA into KGAPB. \\
If KGAPA $=0$, then this momentum is dissipated. \\
\hline Integer $\quad$ Conditional - if NAMGAP(I) $>0$ for channel I \\
\hline$>0$ and $<=$ total number of gaps in model
\end{tabular}

Card 2.4: I, CHAN_GLOBAL, CHAN_OWNER

Card 2.4 is only read if this is a parallel model with multiple CTF input decks.

\begin{tabular}{|c|c|c|c|}
\hline 2.4 & I & {$[-]$} & {$[-]$} \\
\hline \multicolumn{4}{|c|}{ The channel local index (consistent with Card 2.2). } \\
\hline Integer & \multicolumn{3}{|c|}{ Conditional - if this is a parallel run } \\
\hline 2.4 & CHAN_GLOBAL & {$[-]$} & {$[-]$} \\
\hline \multicolumn{4}{|c|}{ The channel global index. } \\
\hline Integer & \multicolumn{3}{|c|}{ Conditional - if this is a parallel run } \\
\hline \multicolumn{4}{|c|}{$>0$ and $<=$ total number of channels in model } \\
\hline 2.4 & CHAN_OWNER & {$[-]$} & {$[-]$} \\
\hline \multicolumn{4}{|c|}{ The index of the domain that owns this channel. } \\
\hline Integer & \multicolumn{3}{|c|}{ Conditional - if this is a parallel run } \\
\hline \multicolumn{4}{|c|}{$>0$ and $<=$ total number of domains in model } \\
\hline
\end{tabular}




\section{Card Group 3}

This card group is read by subroutine READ_CARD_3.

This card is omitted if there are no transverse connections between channels.

The first line indicates the group number: NGROUP $=3$

CARD 3.1: NK, NDUM2, NDUM3, NDUM4, NDUM5, NDUM6, NDUM7, NDUM8, NDUM9, NDUM10, NDUM11, NDUM12, NDUM13, NDUM14

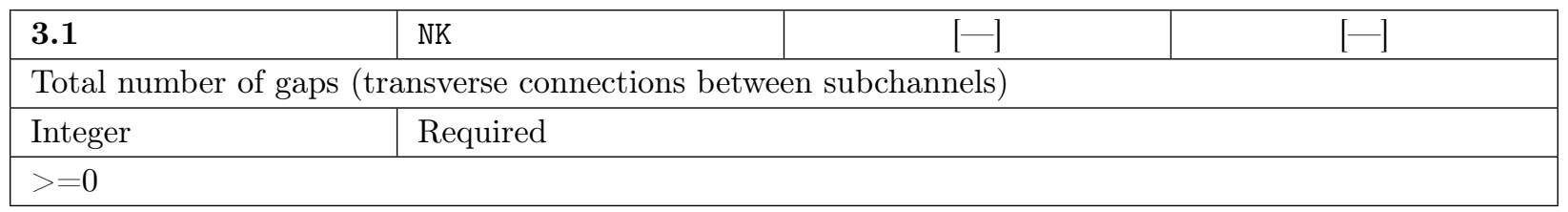

\begin{tabular}{|l|l|l|l|}
\hline 3.1 & NDUM2: NDUM14 & {$[-]$} & {$[-]$} \\
\hline $\begin{array}{l}\text { Not used, but entry is obligatory: } \\
0 \text { - Suggested value }\end{array}$ \\
\hline \begin{tabular}{l} 
Integer \\
\hline 0
\end{tabular}
\end{tabular}

Lines $\underline{\text { CARD } 3.2}$ and $\underline{\text { CARD } 3.3}$ are read in pairs NK times.

CARD 3.2: $K$, IK(K), JK(K), GAPN $(K)$, LENGTH $(K)$, WKR $(K), \operatorname{FWALL}(K)$, IGAPB $(K)$, IGAPA $(K)$, FACTOR $(K)$, $(\operatorname{IGAP}(K, N), \operatorname{JGAP}(K, N), N=1: 3)$

\begin{tabular}{|l|l|c|c|}
\hline $\mathbf{3 . 2}$ & $\mathrm{K}$ & {$[-]$} & {$[-]$} \\
\hline Index of gap & & \\
Note: Gap indices must be entered sequentially from & 1 to NK. Skipping numbers is not permitted. \\
\hline Integer & Required \\
\hline$>0$ &
\end{tabular}




\begin{tabular}{|l|l|c|c|}
\hline $\mathbf{3 . 2}$ & IK $(\mathrm{K})$ & {$[-]$} & {$[-]$} \\
\hline Identification number of the lower-numbered subchannel of the pair connected to gap $\mathrm{K}$ \\
\hline Integer & Required \\
\hline$>0$ and $<=$ number of channels in model \\
\hline
\end{tabular}

\begin{tabular}{|l|l|l|}
\hline 3.2 & JK $(\mathrm{K})$ & {$[-]$} \\
\hline Identification number of the higher-numbered subchannel of the pair connected to gap K \\
\hline Integer & Required \\
\hline$>0$ and $<=$ number of channels in model \\
\hline
\end{tabular}

\begin{tabular}{|c|c|c|c|}
\hline 3.2 & GAPN $(K)$ & {$[\mathrm{m}]$} & [in] \\
\hline \multicolumn{4}{|c|}{ Nominal gap width } \\
\hline Float & Required & & \\
\hline$>0.0$ & & & \\
\hline
\end{tabular}

\begin{tabular}{|c|c|c|c|}
\hline 3.2 & LENGTH (K) & [m] & [in] \\
\hline \multicolumn{4}{|c|}{ Distance between the center of channel $I K(K)$ and the center of subchannel JK(K) } \\
\hline Float & Required & & \\
\hline$>0.0$ & & & \\
\hline
\end{tabular}

\begin{tabular}{|c|c|c|c|}
\hline 3.2 & WKR (K) & {$[\mathrm{m}]$} & [in] \\
\hline \multicolumn{4}{|c|}{$\begin{array}{l}\text { Horizontal pressure loss coefficient (velocity head) for gap } \mathrm{K} \\
\text { Note: this } \zeta \text { value can be specified for the whole gap only. It cannot be specified node-wise in the axial } \\
\text { direction. }\end{array}$} \\
\hline Float & Requirec & & \\
\hline$>=0.0$ & & & \\
\hline
\end{tabular}

\begin{tabular}{|l|l|l|}
\hline 3.2 & FWALL $(\mathrm{K})$ & {$[-]$} \\
\hline Wall friction factor for the gap: \\
0.0 - No walls \\
0.5 - One walls \\
1.0 - Two walls \\
Note: A rod bordering a gap is no wall. If one wall is determined, a wall with the surface area of \\
LENGTH $(K)^{*}$ DXS is assumed to be perpindicular to the gap. \\
\hline Float & Required \\
\hline- &
\end{tabular}




\begin{tabular}{|c|c|c|c|}
\hline 3.2 & $\operatorname{IGAPB}(K)$ & {$[-]$} & {$[-]$} \\
\hline \multicolumn{4}{|c|}{ Index number of the gap in the section below gap $\mathrm{K}$} \\
\hline \multicolumn{4}{|c|}{0 - If there is no gap below gap $\mathrm{K}$} \\
\hline \multicolumn{4}{|c|}{$\begin{array}{l}\text { The velocity of IGAPB (K) convects vertical momentum at node } 1 \text { into (or out of) channel IK (K) and } \\
\text { out of (or into) JK(K). }\end{array}$} \\
\hline Integer & Required & & \\
\hline \multicolumn{4}{|c|}{$>0$ and $<=$ number of gaps in the model } \\
\hline
\end{tabular}

\begin{tabular}{|c|c|c|c|}
\hline 3.2 & $\operatorname{IGAPA}(\mathrm{K})$ & {$[-]$} & {$[-]$} \\
\hline \multicolumn{4}{|c|}{ Index number of the gap in the section above gap $\mathrm{K}$} \\
\hline \multicolumn{4}{|c|}{0 - If there is no gap above gap $\mathrm{K}$} \\
\hline \multicolumn{4}{|c|}{$\begin{array}{l}\text { The velocity of IGAPA (K) convects vertical momentum at the top node of the section into (or out of) } \\
\text { channel IK (K) and out of (or into) JK(K). }\end{array}$} \\
\hline Integer & Required & & \\
\hline
\end{tabular}

Note: The input for FACTOR, IGAP and JGAP is required only if the three-dimensional form of the transverse momentum equation is desired.

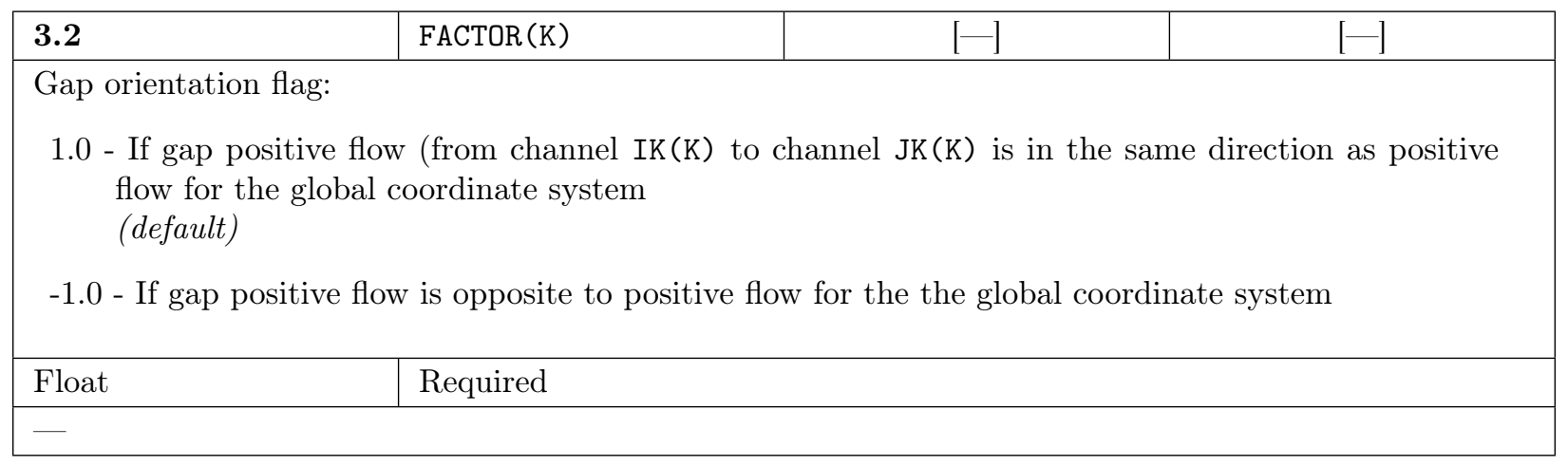




\begin{tabular}{|c|c|c|c|}
\hline 3.2 & $\operatorname{IGAP}(\mathrm{K}, \mathrm{N})$ & {$[-]$} & {$[-]$} \\
\hline \multirow{3}{*}{\multicolumn{4}{|c|}{$\begin{array}{l}\text { Gap numbers facing the IK }(\mathrm{K}) \text { side of gap } \mathrm{K} \text { : } \\
-1 \text { - If the gap faces a wall (lateral momentum will not be transported on this side of the gap). } \\
0 \text { - To dissipate momentum. Has the same effect as }-1 \text { option. Note that not specifically stating how } \\
\text { gaps connect in the lateral direction can lead to erroneous lateral pressure drop calculations (see } \\
\text { the User Guide section of this manual for more information). }\end{array}$}} \\
\hline & & & \\
\hline & & & \\
\hline \multirow{2}{*}{\multicolumn{4}{|c|}{$\begin{array}{l}-(50+\operatorname{IK}(K)) \text { - For gaps facing a pressure source boundary condition, enter the negative sum of } 50 \\
\text { plus the channel that has the pressure source boundary condition. } \\
-(40+\mathrm{IK}(\mathrm{K})) \text { - For gaps facing a mass source boundary condition, enter the negative of the sum of } 40 \\
\text { and the channel that has the mass source boundary condition (note that since the value can only } \\
\text { be between } 40 \text { and } 50 \text {, this means mass flow boundary conditions can only be applied to channels } \\
1 \text { through } 10) \text {. }\end{array}$}} \\
\hline & & & \\
\hline \multicolumn{4}{|c|}{$\begin{array}{l}\text { **Note: Three sets of IGAP, KGAP are required in Card 3.2. If there are fewer gaps to specify, then } \\
\text { enter } 0 \text { until the end of the line is reached }\end{array}$} \\
\hline Integer & Required & & \\
\hline \multicolumn{4}{|l|}{ - } \\
\hline
\end{tabular}

\begin{tabular}{|c|c|c|c|}
\hline 3.2 & $\operatorname{JGAP}(\mathrm{K}, \mathrm{N})$ & {$[-]$} & {$[-]$} \\
\hline \multirow{3}{*}{\multicolumn{4}{|c|}{$\begin{array}{l}\text { Gap numbers facing the JK (K) side of gap K: } \\
\text {-1 - If the gap faces a wall (lateral momentum will not be transported on this side of the gap). } \\
0 \text { - To dissipate momentum. Has the same effect as }-1 \text { option. Note that not specifically stating how } \\
\text { gaps connect in the lateral direction can lead to erroneous lateral pressure drop calculations (see } \\
\text { the User Guide section of this manual for more information). }\end{array}$}} \\
\hline & & & \\
\hline & & & \\
\hline \multirow{2}{*}{\multicolumn{4}{|c|}{$\begin{array}{l}-(50+\mathrm{JK}(\mathrm{K})) \text { - For gaps facing a pressure source boundary condition, enter the negative sum of } 50 \\
\text { plus the channel that has the pressure source boundary condition. } \\
\text { - }(40+\mathrm{JK}(\mathrm{K})) \text { - For gaps facing a mass source boundary condition, enter the negative of the sum of } 40 \\
\text { and the channel that has the mass source boundary condition (note that since the value can only } \\
\text { be between } 40 \text { and } 50 \text {, this means mass flow boundary conditions can only be applied to channels } \\
1 \text { through } 10) \text {. }\end{array}$}} \\
\hline & & & \\
\hline \multicolumn{4}{|c|}{$\begin{array}{l}\text { ** Note: Three sets of IGAP, KGAP are required in Card 3.2. If there are fewer gaps to specify, then } \\
\text { enter } 0 \text { until the end of the line is reached }\end{array}$} \\
\hline Integer & Required & & \\
\hline \multicolumn{4}{|l|}{ - } \\
\hline
\end{tabular}




\begin{tabular}{|l|l|c|c|}
\hline $\mathbf{3 . 3}$ & GMULT $(\mathrm{K})$ & {$[-]$} & {$[-]$} \\
\hline $\begin{array}{l}\text { Number of actual gaps modeled by gap K } \\
\text { There are no internal gaps in a lumped subchannel. Only gaps lying on the interface to the neighboring } \\
\text { lumped subchannel are regarded. }\end{array}$ \\
\hline \multicolumn{3}{|l|}{ Required } \\
\hline \hline $\mathbf{3 . 3}$ & ETANR (K) & {$[-]$} & {$[-]$} \\
\hline Crossflow de-entrainment (deposition) fraction & \\
\hline Float & Required & \\
\hline$>=1.0$ &
\end{tabular}

CARD 3.3.5 is read only if meshing information is being provided (MESH $=1$ from CARD 1.1). It is read NK times (once for each gap in the model).

CARD 3.3.5: $K, X(K), Y(K), N O R M ; K=1: N K$

\begin{tabular}{|l|l|c|c|}
\hline 3.3.5 & K & {$[-]$} & {$[-]$} \\
\hline Gap Number & Conditional - if MESH =1 \\
\hline Integer & \\
\hline Must be a gap index defined in model & \\
\hline
\end{tabular}

\begin{tabular}{|c|c|c|c|}
\hline 3.3 .5 & $\mathrm{X}(\mathrm{K})$ & {$[\mathrm{m}]$} & [in] \\
\hline \multicolumn{4}{|c|}{$\begin{array}{l}\text { X location of the center of the momentum cell that makes up the gap (it will be on the face between } \\
\text { subchannels that are connected by the gap). See Figure } 4.1 \text { for how the global coordinate system is } \\
\text { setup. }\end{array}$} \\
\hline Float & \multicolumn{3}{|c|}{ Conditional - if MESH $=1$} \\
\hline Any & & & \\
\hline
\end{tabular}

\begin{tabular}{|c|c|c|c|}
\hline 3.3.5 & $\mathrm{Y}(\mathrm{K})$ & {$[\mathrm{m}]$} & [in] \\
\hline \multicolumn{4}{|c|}{ Y location of the center of the momentum cell that makes up gap $\mathrm{K}$} \\
\hline Float & \multicolumn{3}{|c|}{ Conditional - if MESH $=1$} \\
\hline Any & & & \\
\hline
\end{tabular}

\begin{tabular}{|c|c|c|c|}
\hline 3.3.5 & NORM & {$[-]$} & {$[-]$} \\
\hline \multicolumn{4}{|c|}{$\begin{array}{l}\text { The direction that flow through the gap travels (it will be either ' } \mathrm{x} \text { ' or ' } \mathrm{y} \text { ' and the orientation is with } \\
\text { regards to the X and Y directions that are shown in Figure 4.1) }\end{array}$} \\
\hline Character & Condi & & \\
\hline
\end{tabular}

CARD 3.4: NLMGAP 


\begin{tabular}{|c|c|c|c|}
\hline 3.4 & NLMGAP & {$[-]$} & {$[-]$} \\
\hline \multirow{2}{*}{\multicolumn{4}{|c|}{$\begin{array}{l}\text { Number of gaps that convect orthogonal transverse momentum } \\
\text { This is required only for the three-dimensional formulation of the transverse momentum equation. } \\
0 \text { - If the three-dimensional form of the transverse momentum equation is not desired }\end{array}$}} \\
\hline & & & \\
\hline Integer & Requires & & \\
\hline \multicolumn{4}{|l|}{$>=0$} \\
\hline
\end{tabular}

Card 3.5 is read NLMGAP times.

This means, only in the case that the three- dimensional form of the transverse momentum equation is applied.

CARD 3.5: $\operatorname{KGAP} 1(\mathrm{~N}), \operatorname{KGAP} 2(\mathrm{~N}), \operatorname{KGAP} 3(\mathrm{~N}) ; \mathrm{N}=1: \operatorname{NLMGAP}$

One set of KGAP1, KGAP2, KGAP3 entries is specified per line. Enter a new line for each set.

\begin{tabular}{|l|l|c|c|}
\hline $\mathbf{3 . 5}$ & KGAP1 $(\mathrm{N})$ & {$[-]$} & {$[-]$} \\
\hline Index number of the gap whose velocity transports transverse momentum from one gap to another \\
\hline Integer & Conditional - if NLMGAP $\neq 0$ \\
\hline \multicolumn{2}{|l|}{ Must be a gap index defined in model } \\
\hline
\end{tabular}

\begin{tabular}{|c|c|c|c|}
\hline 3.5 & KGAP2 (N) & {$[-]$} & {$[-]$} \\
\hline \multicolumn{4}{|c|}{$\begin{array}{l}\text { Index number of the gap which receives the transverse momentum convected by the positive velocity } \\
\text { of gap KGAP } 1 \\
\text { A number } \neq 0 \text { must be entered. }\end{array}$} \\
\hline Integer & Condition & & \\
\hline
\end{tabular}

\begin{tabular}{|c|c|c|c|}
\hline 3.5 & KGAP3 (N) & {$[-]$} & {$[-]$} \\
\hline \multicolumn{4}{|c|}{$\begin{array}{l}\text { Index number of the gap that the positive velocity of KGAP1 transports transverse momentum out of. } \\
\text { (The positive velocity of KGAP1 transports momentum from KGAP3 to KGAP2. } \\
\text { Note: The negative velocity of KGAP1 will transport transverse momentum in the opposite direction; } \\
\text { i.e., from KGAP2 into KGAP3.) } \\
\text { A number } \neq 0 \text { must be entered. }\end{array}$} \\
\hline Integer & Condition & & \\
\hline
\end{tabular}

Card 3.6 is read only if this is a parallel model. This is not required, and if it is not provided by the user, then CTF will determine gap domain owners internally.

CARD 3.6: K, GAP_GLOBAL, GAP_OWNER

One set is entered per line. 


\begin{tabular}{|l|l|c|c|}
\hline 3.6 & $\mathrm{K}$ & {$[-]$} & {$[-]$} \\
\hline Gap index number (consistent with 3.2 above). \\
\hline Integer & Conditional if this is a parallel run \\
\hline Must be a gap index defined in model \\
\hline
\end{tabular}

\begin{tabular}{|l|l|c|c|}
\hline 3.6 & GAP_GLOBAL & {$[-]$} & {$[-]$} \\
\hline Global index of the gap in the model. \\
\hline Integer & Conditional if this is a parallel run \\
\hline$>0$
\end{tabular}

\begin{tabular}{|l|l|l|l|}
\hline 3.6 & GAP_OWNER & {$[-]$} & {$[-]$} \\
\hline Index of the domain that owns this gap. & \\
\hline Integer & Conditional if this is a parallel run \\
\hline$>0$ and less than number of domains in model \\
\hline
\end{tabular}




\section{Card Group 4}

This card group is read by subroutine READ_CARD_4.

The first line indicates the group number: NGROUP $=4$

CARD 4.1: NSECTS, NSIM, IREBAL, NDUM4, NDUM5, NDUM6, NDUM7, NDUM8, NDUM9, NDUM10, NDUM11, NDUM12, NDUM13, NDUM14

\begin{tabular}{|l|l|c|c|}
\hline $\mathbf{4 . 1}$ & NSECTS & {$[-]$} & {$[-]$} \\
\hline Number of sections & & \\
\hline Integer & Required & \\
\hline$>0$ &
\end{tabular}

\begin{tabular}{|c|c|c|c|}
\hline 4.1 & NSIM & {$[-]$} & {$[-]$} \\
\hline \multicolumn{4}{|c|}{ Number of simultaneous solution groups: } \\
\hline \multicolumn{4}{|c|}{1 - Direct Gaussian elimination (for pressure equation) } \\
\hline \multicolumn{4}{|c|}{$<1$ - Iterative Gauss-Seidel solver (for pressure equation) } \\
\hline Integer & Requ & & \\
\hline
\end{tabular}

\begin{tabular}{|c|c|c|c|}
\hline 4.1 & IREBAL & {$[-]$} & {$[-]$} \\
\hline \multicolumn{4}{|c|}{ Rebalancing option for iteration control (acceleration of convergence): } \\
\hline \multicolumn{4}{|c|}{0 - No rebalancing } \\
\hline \multicolumn{4}{|c|}{1 - Rebalancing } \\
\hline \multicolumn{4}{|c|}{ Note: If NSIM $=1$, IREBAL is set to 0.} \\
\hline Integer & Required & & \\
\hline- & & & \\
\hline
\end{tabular}




\begin{tabular}{|c|c|c|c|}
\hline 4.1 & NDUM4 : NDUM14 & {$[-]$} & {$[-]$} \\
\hline \multicolumn{4}{|c|}{$\begin{array}{l}\text { Not used, but entry is obligatory: } \\
0 \text { - Suggested value }\end{array}$} \\
\hline Integer & Required & & \\
\hline
\end{tabular}

Note: Within a simultaneous solution group, different solvers can be selected by means of parameter ISOL in CARD 1.1. But in the case of the Krylov solver (ISOL =1) it is not recommended to divide the computational domain into more than one simultaneous solution group.

Cards CARD 4.2, CARD 4.3, and CARD 4.4 are read in a group NSECTS times.

CARD 4.2: ISEC, NCHH, NONODE, DXS (ISEC, 1), IVARDX

\begin{tabular}{|c|c|c|c|}
\hline 4.2 & ISEC & {$[-]$} & {$[-]$} \\
\hline \multicolumn{4}{|c|}{$\begin{array}{l}\text { Section number } \\
\text { Begin with section number } 1 \text { on the bottom of the vessel and proceed towards the top, incrementing } \\
\text { ISEC by } 1 .\end{array}$} \\
\hline Integer & Required & & \\
\hline
\end{tabular}

\begin{tabular}{|l|l|c|c|}
\hline $\mathbf{4 . 2}$ & NCHN & {$[-]$} & {$[-]$} \\
\hline Number of channels in section ISEC & \\
\hline Integer & Required \\
\hline$>0$ and $<=$ total number of channels in model \\
\hline
\end{tabular}

\begin{tabular}{|c|c|c|c|}
\hline 4.2 & NONODE & {$[-]$} & {$[-]$} \\
\hline \multicolumn{4}{|c|}{$\begin{array}{l}\text { Number of vertical levels (continuity mesh cells without auxiliary lower or upper boundary cells) in } \\
\text { section ISEC }\end{array}$} \\
\hline Integer & Required & & \\
\hline$>0$ & & & \\
\hline
\end{tabular}

\begin{tabular}{|c|c|c|c|}
\hline 4.2 & DXS (ISEC , 1) & [m] & [in] \\
\hline \multicolumn{4}{|c|}{ Vertical mesh cell length in section ISEC } \\
\hline Float & Required & & \\
\hline$>0.0$ & & & \\
\hline
\end{tabular}




\begin{tabular}{|c|c|c|c|}
\hline 4.2 & IVARDX & {$[-]$} & {$[-]$} \\
\hline \multicolumn{4}{|c|}{ Flag for variable node length in section ISEC: } \\
\hline \multicolumn{4}{|c|}{0 - Constant mesh cell length (default) } \\
\hline \multicolumn{4}{|c|}{$>0$ - Read IVARDX pairs in variable $\Delta \mathrm{x}$ table (see Card 4.3 ) } \\
\hline Integer & Required & & \\
\hline$>0$ & & & \\
\hline
\end{tabular}

Card 4.3 is only read if IVARDX $>0$.

Up to 5 sets of (JLEV, VARDX) may be entered. If IVARDX is greater than 5 , repeat CARD 4.3 until IVARDX pairs has been entered. If IVARDX is less than 5 enter $(0 ; 0.0)$ until 5 sets of (JLEV, VARDX) have been entered.

CARD 4.3: JLEV(I), $\operatorname{VARDX}(I) ; I=1: \operatorname{IVARDX}$

\begin{tabular}{|l|l|l|}
\hline $\mathbf{4 . 3}$ & JLEV (I) & {$[-]$} \\
\hline $\begin{array}{l}\text { Last axial level in section ISEC to have a node length of VARDX (I) } \\
\text { JLEV (IVARDX) must be greater than or equal to NONODE }+1\end{array}$ \\
\hline Integer & Conditional - if IVARDX >0 \\
\hline-
\end{tabular}

\begin{tabular}{|l|l|c|c|}
\hline $\mathbf{4 . 3}$ & VARDX (I) & {$[\mathrm{m}]$} & [in] \\
\hline Axial mesh cell length & & \\
\hline Float & Conditional - if IVARDX >0 & \\
\hline$>0.0$ &
\end{tabular}

Card 4.4 is read NCHN times for section ISEC. If PPV $>=7$ on Card 1.1, then you must specify how many channels are connected above and how many are connected below. If PPV $<7$, a hard-coded number of 6 above and below connections must be specified. The PPV $>=7$ option allows the user to connect a channel to any arbitrary number of channels above or below. Keep the line length below the maximum allowed length by entering large numbers of channels on new lines.

CARD 4.4: $\operatorname{PPV}<7: \mathrm{I}, \operatorname{KCHANA}(\mathrm{I}, \mathrm{J}) ; \mathrm{J}=2: 7, \operatorname{KCHANB}(\mathrm{I}, \mathrm{J}) ; \mathrm{J}=2: 7 \mathrm{PPV}>=7: \mathrm{I}, \mathrm{NCHA}, \operatorname{KCHANA}(\mathrm{I}, \mathrm{J})$; $\mathrm{J}=2: \mathrm{NCHA}, \mathrm{NCHB}, \operatorname{KCHANB}(I, J) ; \mathrm{J}=2: \mathrm{NCHB}$

\begin{tabular}{|l|l|c|c|}
\hline $\mathbf{4 . 4}$ & I & {$[-]$} & {$[-]$} \\
\hline Identification number of a channel in section ISEC & \\
\hline Integer & Required \\
\hline Must be a channel index in model \\
\hline
\end{tabular}




\begin{tabular}{|l|l|c|c|}
\hline $\mathbf{4 . 4}$ & NCHA & {$[-]$} & {$[-]$} \\
\hline Only enter if PPV $>=7$. & Specifies the number of channels connected to this one in the section above. \\
\hline Integer & Enter if PPV $>=7$ \\
\hline$>=0$ & \\
\hline
\end{tabular}

\begin{tabular}{|c|c|c|c|}
\hline 4.4 & $\operatorname{KCHANA}(\mathrm{I}, \mathrm{J})$ & {$[-]$} & {$[-]$} \\
\hline \multicolumn{4}{|c|}{$\begin{array}{l}\text { Indices of channels in the section above ISEC that connect to channel I. If channel I does not connect } \\
\text { to any channels above, enter I in KCHANA }(\mathrm{I}, 2) \text {. A maximum of } 6 \text { channels can be connected to channel } \\
\text { I when PPV }<7 \text {. If using PPV }<7 \text { and the number of channels connected to channel I is less than } 6 \text {, } \\
\text { enter } 0 \text { until } 6 \text { KCHANA values have been entered. When using PPV }>=7 \text {, enter NCHA channels }\end{array}$} \\
\hline Integer & Required & & \\
\hline
\end{tabular}

\begin{tabular}{|l|l|l|l|}
\hline $\mathbf{4 . 4}$ & NCHB & {$[-]$} & {$[-]$} \\
\hline Only enter if PPV $>=7$. & Number of channels connected to this one in the section below. \\
\hline Integer & Enter if PPV $>=7$ \\
\hline$>=0$ & \\
\hline
\end{tabular}

\begin{tabular}{l|l|c|}
\hline 4.4 & $\operatorname{KCHANB}(\mathrm{I}, \mathrm{J})$ & {$[-]$} \\
\hline Indices of channels in the section below ISEC that connect to channel I. If channel I does not connect \\
to any channels below, enter I in KCHANB $(\mathrm{I}, 2)$. A maximum of 6 channels can be connected to channel \\
I if using PPV $<7$. If using PPV $<7$ and the number of channels connected to channel I is less than 6, \\
enter 0 until 6 KCHANB values have been entered. Enter NCHB channels if using PPV $>=7$. \\
\hline Integer & Required \\
\hline \\
Must be a channel index defined in model
\end{tabular}

\section{CARD 4.5: IWIDE}

\begin{tabular}{|c|c|c|c|}
\hline 4.5 & IWIDE & {$[-]$} & {$[-]$} \\
\hline \multicolumn{4}{|c|}{$\begin{array}{l}\text { Maximum difference between the index numbers of adjacent cells (vertical or transversal, respectively) } \\
\text { in a simultaneous solution group (For the simplest case of only one simultaneous solution group, one } \\
\text { section, and no sub-divided subchannels âĂŞ i.e. only one mesh cell per subchannel radial direction } \\
\text { âAŞ IWIDE is equal to the number of subchannels). }\end{array}$} \\
\hline Integer & Require & & \\
\hline
\end{tabular}

CARD 4.6: $\operatorname{MSIM(I);~I~=~} 1: \operatorname{NSIM}$

Twelve values are entered per card. If NSIM is greater than 12, repeat CARD 4.6 until NSIM values have been entered. 


\begin{tabular}{|l|l|c|}
\hline $\mathbf{4 . 5}$ & MSIM(I) & {$[-]$} \\
\hline $\begin{array}{l}\text { Last cell number in each simultaneous solution group. } \\
\text { This is the total number of continuity mesh cells within the simultaneous solution group I (number of } \\
\text { subchannels times the number of vertical layers NONODE). }\end{array}$ \\
\hline Integer & Required \\
\hline$>0$ &
\end{tabular}




\section{Card Group 5}

This card group is read by subroutine SETIN.

Note: The input for this group allows the user to specify vertical variations in the continuity area, momentum area, or wetted perimeter for channels, and in the transverse width for gaps. It can be omitted, if such variations are not needed.

The first line indicates the group number: NGROUP $=5$

CARD 5.1: NAFACT, NAXL, NDUM3, NDUM4, NDUM5, NDUM6, NDUM7, NDUM8, NDUM9, NDUM10, NDUM11, NDUM12, NDUM13, NDUM14

\begin{tabular}{|l|l|l|l|}
\hline $\mathbf{5 . 1}$ & NAFACT & {$[-]$} & {$[-]$} \\
\hline Total number of geometry variation tables & & \\
\hline Integer & Required & \\
\hline$>0$ &
\end{tabular}

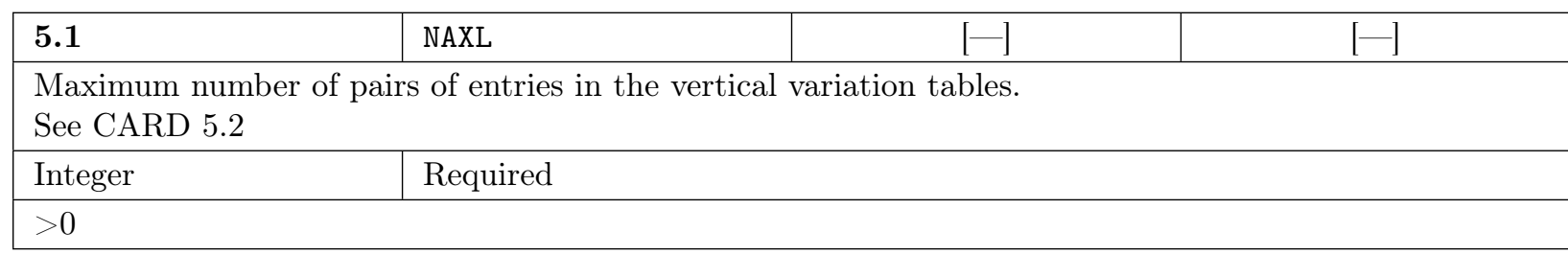

\begin{tabular}{l|l|l|l|}
\hline $\mathbf{5 . 1}$ & NDUM3: NDUM14 & {$[-]$} & {$[-]$} \\
\hline $\begin{array}{l}\text { Not used, but entry is obligatory: } \\
0 \text { - Suggested value }\end{array}$ & \\
\hline \multicolumn{2}{|l|}{ Integer } & Required \\
\hline 0 &
\end{tabular}

Card 5.2 is read NAFACT times. The tables are numbered sequentially in the code.

Eight pairs of (JAXL, AFACT) are entered per card. The tables are numbered sequentially in the code. All 
entered tables must have a full set of NAXL pairs of (JAXL, AFACT) data.

CARD 5.2: $\operatorname{JAXL}(I, N), \operatorname{AFACT}(I, N) ; N=1: \operatorname{NAXL}(I)$

\begin{tabular}{|c|c|c|c|}
\hline 5.2 & $\operatorname{JAXL}(I, N)$ & {$[-]$} & {$[-]$} \\
\hline \multicolumn{4}{|c|}{ Axial node number at which to apply the geometry variation factor for table I, point $\mathrm{N}$} \\
\hline Integer & Required & & \\
\hline
\end{tabular}

\begin{tabular}{|c|c|c|c|}
\hline 5.2 & $\operatorname{AFACT}(I, N)$ & {$[-]$} & {$[-]$} \\
\hline \multicolumn{4}{|c|}{$\begin{array}{l}\text { Geometry variation factor for table } \mathrm{I} \text {, point } \mathrm{N} \\
\text { Whether } \operatorname{AFACT}(\mathrm{I}, \mathrm{N}) \text { is applied to channel areas, wetted perimeter, or gap width, is specified in CARD } \\
6.2 \text {. }\end{array}$} \\
\hline Float & Required & & \\
\hline$>0.0$ & & & \\
\hline
\end{tabular}




\section{Card Group 6}

This card group is read by subroutine SETIN.

The first line indicates the group number: NGROUP $=6$

CARD 6.1: N1, NDUM2, NDUM3, NDUM4, NDUM5, NDUM6, NDUM7, NDUM8, NDUM9, NDUM10, NDUM11, NDUM12, NDUM13, NDUM14

\begin{tabular}{|l|l|c|c|}
\hline $\mathbf{6 . 1}$ & $\mathrm{N} 1$ & {$[-]$} & {$[-]$} \\
\hline \multicolumn{2}{|l|}{ Total number of channel and gap variation table cards to be read in CARD 6.2 } \\
\hline Integer & Required \\
\hline$>0$ & \\
\hline
\end{tabular}

\begin{tabular}{|l|l|l|l|}
\hline $\mathbf{6 . 1}$ & NDUM2: NDUM14 & {$[-]$} & {$[-]$} \\
\hline $\begin{array}{l}\text { Not used, but entry is obligatory: } \\
0 \text { - Suggested value }\end{array}$ \\
\hline Integer & Required & \\
\hline 0 &
\end{tabular}

Card 6.2 is read $\mathrm{N} 1$ times.

CARD 6.2: IACT, IAMT, IPWT, ICRG(M); M = 1:12

\begin{tabular}{|c|c|c|c|}
\hline 6.2 & IACT & {$[-]$} & {$[-]$} \\
\hline \multicolumn{4}{|c|}{ Table number for geometry variation: } \\
\hline \multicolumn{4}{|c|}{$>0$ - For subchannel continuity area variation } \\
\hline \multicolumn{4}{|c|}{$<0$ - For gap width variation } \\
\hline Integer & Required & & \\
\hline- & & & \\
\hline
\end{tabular}




\begin{tabular}{l|l|l|l|}
\hline $\mathbf{6 . 2}$ & IAMT & {$[-]$} & {$[-]$} \\
\hline $\begin{array}{l}\text { Table number for subchannel momentum area variation: } \\
0 \text { - If IACT }<0\end{array}$ & \\
\hline Integer & Required & \\
\hline-
\end{tabular}

\begin{tabular}{|l|l|l|l|}
\hline $\mathbf{6 . 2}$ & IPWT & {$[-]$} & {$[-]$} \\
\hline $\begin{array}{l}\text { Table number for subchannel wetted perimeter variation: } \\
0 \text { - If IACT }<0\end{array}$ & \\
\hline Integer & Required \\
\hline-
\end{tabular}

\begin{tabular}{|c|c|c|c|}
\hline 6.2 & ICRG (M) & {$[-]$} & {$[-]$} \\
\hline \multicolumn{4}{|c|}{$\begin{array}{l}\text { Index numbers of the subchannels (if IACT }>0 \text { ) or gaps (if IACT }<0 \text { ) that the tables specified in IACT, } \\
\text { IAMT, and IPWT are to be applied to. } \\
\text { Up to } 12 \text { channels or gaps may be specified per card. If the number of specified channels or gaps is } \\
\text { greater than } 12 \text {, repeat CARD } 6.2 \text { until all values have been entered. If the number of specified channels } \\
\text { or gaps is less than } 12 \text {, enter } 0 \text { until } 12 \text { values have been entered. }\end{array}$} \\
\hline Integer & Required & & \\
\hline
\end{tabular}




\section{Card Group 7}

This card group is read by subroutine READ_CARD_7.

This card group may be omitted if there are no spacers.

The first line indicates the group number: NGROUP $=7$

CARD 7.1: NCD, NGT, IFGQF, IFSDRP, IFESPV, IFTPE, IGTEMP, NFBS, IFCD, IXFLOW, NDUM11, NDUM12, NDUM13, NDUM14

\begin{tabular}{l|l|l|l|}
\hline $\mathbf{7 . 1}$ & NCD & {$[-]$} & {$[-]$} \\
\hline $\begin{array}{l}\text { Number of lines in CARD } 7.2 \text { specifying local spacer pressure loss coefficients. These include vertical } \\
\text { pressure losses only. Transverse pressure losses are specified in CARD GROUP 3. }\end{array}$ \\
$\begin{array}{ll}\text { Integer } & \text { Required } \\
>=0 & \end{array}$ \\
\hline
\end{tabular}

\begin{tabular}{|c|c|c|c|}
\hline 7.1 & NGT & {$[-]$} & {$[-]$} \\
\hline \multirow{3}{*}{\multicolumn{4}{|c|}{$\begin{array}{l}\text { Number of grid types with special geometrical specification: } \\
0 \text { - If spacers are defined via pressure loss coefficients CDL in CARD } 7.2 \text { only. } \\
>0 \text { - If spacers are defined via geometrically- modeled blockages in CARD } 7.3 \text { through CARD } 7.9 \text { or } \\
\quad \text { if spacers are modeled as heat transfer enhancements on CARD } 7.1 .6\end{array}$}} \\
\hline & & & \\
\hline & & & \\
\hline Integer & Required & & \\
\hline \multicolumn{4}{|l|}{$>=0$} \\
\hline
\end{tabular}




\begin{tabular}{|l|l|c|c|}
\hline $\mathbf{7 . 1}$ & IFGQF & {$[-]$} & {$[-]$} \\
\hline \multicolumn{2}{|l|}{ Flag for grid quench front model: } & \\
$\geq 1$ - On & \\
$<1$ - Off & & \\
\hline Integer & Required & \\
\hline- &
\end{tabular}

\begin{tabular}{|l|l|l|l|}
\hline $\mathbf{7 . 1}$ & IFSDRP & {$[-]$} & {$[-]$} \\
\hline $\begin{array}{l}\text { Flag for small drop model: } \\
\geq 1-\text { On } \\
<1-\text { Off }\end{array}$ & \\
\hline Integer & Required & \\
\hline- & \\
\hline
\end{tabular}

\begin{tabular}{|c|c|c|c|}
\hline 7.1 & IFESPV & {$[-]$} & {$[-]$} \\
\hline \multicolumn{4}{|c|}{ Flag for grid convective enhancement: } \\
\hline \multicolumn{4}{|l|}{$0-$ Off } \\
\hline \multicolumn{4}{|c|}{1 - On for single phase vapor only } \\
\hline \multicolumn{4}{|c|}{$\begin{array}{l}2 \text { - On for both single-phase vapor and liquid (multiplied by the HTC calculated by Dittus-Boelter } \\
\text { only) }\end{array}$} \\
\hline \multicolumn{4}{|c|}{$\begin{array}{l}3 \text { - Simple grid modeling option with grid enhancement for both phases. } \\
\text { If selected, do not enter CARD } 7.3 \text { through CARD 7.5. Instead, specify the grid enhancement } \\
\text { locations on CARD 7.1.6 }\end{array}$} \\
\hline Integer & Required & & \\
\hline- & & & \\
\hline
\end{tabular}

\begin{tabular}{|c|c|c|c|}
\hline 7.1 & IFTPE & {$[-]$} & {$[-]$} \\
\hline \multicolumn{4}{|c|}{ Flag for two-phase enhanceent of dispersed flow heat transfer: } \\
\hline \multicolumn{4}{|c|}{$\geq 1-\mathrm{On}$} \\
\hline \multicolumn{4}{|c|}{$<1-$ Off } \\
\hline Integer & Requir & & \\
\hline
\end{tabular}




\begin{tabular}{|c|c|c|c|}
\hline 7.1 & IGTEMP & {$[-]$} & {$[-]$} \\
\hline \multicolumn{4}{|c|}{ Flag for grid quench calculations (see CARD 7.8): } \\
\hline \multicolumn{4}{|c|}{$\geq 1-$ On } \\
\hline \multicolumn{4}{|c|}{$<1$ - Off } \\
\hline Integer & Required & & \\
\hline - & & & \\
\hline
\end{tabular}

\begin{tabular}{|l|l|c|c|}
\hline $\mathbf{7 . 1}$ & NFBS & {$[-]$} & {$[-]$} \\
\hline Number of flow blockages & & \\
\hline Integer & Required & \\
\hline$>=0$ & \\
\hline
\end{tabular}

\begin{tabular}{|c|c|c|c|}
\hline 7.1 & IFCD & {$[-]$} & {$[-]$} \\
\hline \multicolumn{4}{|c|}{ Flag for internal calculation of grid pressure loss coefficients: } \\
\hline \multicolumn{4}{|c|}{$\geq 1-$ On } \\
\hline \multicolumn{4}{|c|}{$<1-$ Off } \\
\hline Integer & Required & & \\
\hline- & & & \\
\hline
\end{tabular}

\begin{tabular}{|l|l|c|c|}
\hline 7.1 & IXFLOW & {$[-]$} & {$[-]$} \\
\hline
\end{tabular}

Flag for modeling lateral-exhange cross-flow effects caused by the spacer grids (see CARD 7.9):

0 - No special modeling

1 - Directed cross-flow model

2 - Enhanced turbulence mixing model

3 - Both models included

\begin{tabular}{|l|l|}
\hline Integer & Required \\
\hline- & \\
\hline
\end{tabular}

\begin{tabular}{|l|l|l|l|}
\hline $\mathbf{7 . 1}$ & NDUM11: NDUM14 & {$[-]$} & {$[-]$} \\
\hline $\begin{array}{l}\text { Not used, but entry is obligatory: } \\
0 \text { - Suggested value }\end{array}$ & \\
\hline Integer & Required \\
\hline 0 &
\end{tabular}


Card 7.1.5 is read only if IFESPV $=1,2$, or 3. The card is read NGT times. The terms C1, C2, C3, C4, A, and PHI in Card 7.1.5 refer respectively to the coefficients $a, b, c, d, A$, and $\Phi$ in

$$
\frac{N u}{N u_{0}}=\left[1+a \epsilon^{2} \exp \left[b \frac{x}{D}\right]\right]\left[1+A^{2} \tan ^{2}(\Phi) \exp \left[c \frac{x}{D}\right]\right]^{d} .
$$

This equation represents the mixing-vane form of the Yao-Hochreiter-Leech formula, with the original reference values for all coefficients shown in the Card 7.1.5 input description. However, it has been observed that the mixing-vane component of the equation does not produce accurate results. Therefore, it is suggested that the C3, C4, A, and PHI terms be set to 0.0 to disable that portion of the correlation. Additional details on the Yao-Hochreiter-Leech formula and its implementation in CTF are given in the CTF Theory Manual [1].

SIMPLE GRID MODELING: If not requiring the advanced models offered by Cards 7.3 through 7.5, the simple grid modeling option can be utilized by setting IFESPV to 3 on Card 7.1, setting grid type parameters on Card 7.1.5, setting grid enhancement locations on Card 7.1.6, and entering grid loss coefficients on Card 7.2 .

Card 7.1.5 is read NGT times (if IFESPV >0). Enter parameters for each grid on a separate line. Line 1 will be Grid ID 1, line 2 will be Grid ID 2, and so on.

CARD 7.1.5: $\mathrm{C} 1(\mathrm{~N}), \mathrm{C} 2(\mathrm{~N}), \mathrm{C} 3(\mathrm{~N}), \mathrm{C} 4(\mathrm{~N}), \mathrm{A}(\mathrm{N}), \mathrm{PHI}(\mathrm{N}), \mathrm{N}=1: \mathrm{NGT}$

\begin{tabular}{|l|l|l|}
\hline 7.1.5 & $\begin{array}{l}\text { C1 }(\mathrm{N}), \mathrm{C} 2(\mathrm{~N}), \\
\mathrm{C} 3(\mathrm{~N}), \mathrm{C} 4(\mathrm{~N})\end{array}$ & {$[-]$} \\
\hline Coefficients in the Yao-Hochreiter-Leech formula & \\
C1 - Reference value is 5.55 (enter $>=0.0)$ \\
C2 - Reference value is $-0.13($ enter $<=0.0)$ \\
C3 - Reference value is $-0.034($ enter $<=0.0)$ \\
C4 - Reference value is 0.4 (enter $>=0.0)$ \\
\hline Float $[4]$ \\
\hline-
\end{tabular}

\begin{tabular}{|l|l|c|c|}
\hline $\mathbf{7 . 1 . 5}$ & $\mathrm{A}(\mathrm{N})$ & {$[-]$} & {$[-]$} \\
\hline \multicolumn{2}{|l|}{ The blockage ratio normal to the flow caused by the mixing vanes } \\
\hline Float & Conditional - if IFESPV $>0$ \\
\hline$>=0.0$ and $<1.0$ & \\
\hline
\end{tabular}

\begin{tabular}{|l|l|c|c|}
\hline $\mathbf{7 . 1 . 5}$ & PHI (N) & [degrees] & [degrees] \\
\hline Angle of the vanes with respect to the flow direction & \\
\hline Float & Conditional - if IFESPV $>0$ \\
\hline$>=0.0$ and $<=90.0$ & \\
\hline
\end{tabular}


Note: If IFESPV $=3$, the following parameters must be included in Card 7.1.5 as well:

\begin{tabular}{|l|l|l|l|}
\hline $\mathbf{7 . 1 . 5}$ & ABLOC $(\mathrm{N})$ & {$[-]$} & {$[-]$} \\
\hline Blockage ratio of the grid straps & & \\
\hline Float & Conditional - if IFESPV $=3$ \\
\hline$>=0.0$ and $<1.0$ & \\
\hline
\end{tabular}

\begin{tabular}{|l|l|l|l|}
\hline 7.1.5 & SPBLOC $(\mathrm{N})$ & {$[-]$} & {$[-]$} \\
\hline Blockage ratio of the grid springs & \\
\hline Float & Conditional - if IFESPV $=3$ \\
\hline$>=0.0$ and $<1.0$ & \\
\hline
\end{tabular}

Card 7.1.6 is read only if IFESPV $=3$. It specifies the location of each enhancement in the model. CTF will continue reading lines of Card 7.1.6 until it encounters a negative number on a new line.

CARD 7.1.6: GRIDTYPE, J, CHANNEL (M); M $=1: 12$

\begin{tabular}{|l|l|l|l|}
\hline 7.1.6 & GRIDTYPE & {$[-]$} & {$[-]$} \\
\hline The grid type ID of this grid. Data for each grid type were entered on Card 7.1.5. \\
\hline Integer & Conditional - if IFESPV = 3 \\
\hline- & \\
\hline
\end{tabular}

\begin{tabular}{|l|l|l|l|}
\hline 7.1.6 & $\mathrm{J}$ & {$[-]$} & {$[-]$} \\
\hline The axial momentum cell in which this grid type is located \\
\hline Integer & Conditional - if IFESPV $=3$ \\
\hline$>0$ and $<=$ number of momentum levels in channel \\
\hline
\end{tabular}

\begin{tabular}{l|l|c|c|}
\hline 7.1.6 & CHANNEL (M) & {$[-]$} & {$[-]$} \\
\hline $\begin{array}{l}\text { The channel in which this grid type is located. Enter } 12 \text { channels on one line. If the grid resides in } \\
\text { fewer than } 12 \text { channels for one axial level, enter } 0 \text { until } 12 \text { entries have been made. }\end{array}$ \\
\hline Integer & Conditional - if IFESPV =3 \\
\hline Must be a channel in the model
\end{tabular}

**Note: A negative number must be entered on a new line in order to terminate Card 7.1.6.

CARD 7.2 is read NCD times. Do not enter this card if IFESPV $=1$ or 2 . Enter it if IFESPV $=0$ or 3 . Note that if IFESPV is used, a loss coefficient for each location in the model that contains a grid type that will cause a mixing enhancement in the flow MUST be entered. For example, if Grid ID 1 at axial level 5 is placed in channel 3, a loss coefficient in axial level 5 and channel 3 that corresponds to that grid type must be entered. While fewer loss coefficients than there are enhancements to the flow cannot be entered, it is permissible to enter more loss coefficients than there are enhancements.

CARD 7.2: CDL, J, ICDUM(I); I $=1: 12$ 


\begin{tabular}{|l|l|l|l|}
\hline $\mathbf{7 . 2}$ & CDL & {$[-]$} & {$[-]$} \\
\hline Pressure loss coefficient & (vertical) of spacer & \\
\hline Float & Conditional - if IFESPV $=0$ or 3 \\
\hline$>=0.0$ & \\
\hline
\end{tabular}

\begin{tabular}{|c|c|c|c|}
\hline 7.2 & $\mathrm{~J}$ & {$[-]$} & {$[-]$} \\
\hline \multicolumn{4}{|c|}{$\begin{array}{l}\text { Node number at which the pressure loss coefficient is applied. } \\
\text { Note: The vertical node number is relative to the beginnning of the section containing the channel(s) } \\
\text { listed in ICDUM(I). }\end{array}$} \\
\hline Integer & & & \\
\hline
\end{tabular}

\begin{tabular}{|c|c|c|c|}
\hline 7.2 & ICDUM (I) & {$[-]$} & {$[-]$} \\
\hline \multicolumn{4}{|c|}{$\begin{array}{l}\text { Index number of channel to which the pressure loss coefficient will be applied at axial node } \mathrm{J} \text {. } \\
\text { Up to } 12 \text { channels may use the specified pressure loss coefficient CDL at vertical node J. If the number } \\
\text { of channels is fewer than } 12 \text {, enter } 0 \text { until } 12 \text { values have been entered. }\end{array}$} \\
\hline Integer & Condition & & \\
\hline
\end{tabular}

Note: If pressure loss coefficients CDL are specified in CARD \%.2, then CARD $\% .3$ through CARD 7.9 must be omitted. (Pressure losses are specified either by $\zeta$ values or by geometrically modeled flow blockages.)

CARD 7.3, CARD 7.4, and CARD 7.5 are read in groups NG times, where NG $=1$ :NGT. Only enter this group if $\mathrm{IFESPV}=1$ or 2.

CARD 7.3: ING, NGAL (NG), NGCL(NG), IGMAT(NG), GLOSS(NG), GABLOC(NG), GLONG(NG), GPERIM(NG), $\operatorname{SPBLOC}(\mathrm{NG}), \operatorname{TPROBE}(\mathrm{NG})$

\begin{tabular}{|c|c|c|c|}
\hline 7.3 & ING & {$[-]$} & {$[-]$} \\
\hline \multicolumn{4}{|c|}{ Grid type number (must be sequential starting with 1 ) } \\
\hline Integer & \multicolumn{3}{|c|}{ Conditional - if IFESPV $=1$ or 2} \\
\hline \multicolumn{4}{|l|}{ - } \\
\hline 7.3 & NGAL (NG) & {$[-]$} & {$[-]$} \\
\hline \multicolumn{4}{|c|}{$\begin{array}{l}\text { Number of axial locations for grid type ING } \\
\text { Maximum }=16\end{array}$} \\
\hline Integer & \multicolumn{3}{|c|}{ Conditional - if IFESPV $=1$ or 2} \\
\hline \multicolumn{4}{|c|}{$>0$ and $<=16$} \\
\hline 7.3 & NGCL (NG) & {$[-]$} & {$[-]$} \\
\hline \multicolumn{4}{|c|}{ Number of channels containing grid ING at levels NGAL } \\
\hline Integer & \multicolumn{3}{|c|}{ Conditional - if IFESPV $=1$ or 2} \\
\hline$>0$ & & & \\
\hline
\end{tabular}




\begin{tabular}{|l|l|l|l|}
\hline $\mathbf{7 . 3}$ & IGMAT $(\mathrm{NG})$ & {$[-]$} & {$[-]$} \\
\hline Grid material type index corresponding to material types in CARD GROUP 10 \\
\hline Integer & Conditional - if IFESPV = 1 or 2 \\
\hline-
\end{tabular}

\begin{tabular}{|c|c|c|c|}
\hline 7.3 & GLOSS (NG) & {$[-]$} & {$[-]$} \\
\hline \multicolumn{4}{|c|}{ Pressure loss coefficient multiplier: } \\
\hline \multicolumn{4}{|c|}{ 1.0 - Suggested value for round-egde grids } \\
\hline \multicolumn{4}{|c|}{1.4 - Suggested value for square-edge grids } \\
\hline Float & Conditiona & & \\
\hline$>=1.0$ & & & \\
\hline
\end{tabular}

\begin{tabular}{|l|l|l|l|}
\hline $\mathbf{7 . 3}$ & GABLOC $(\mathrm{NG})$ & {$[-]$} & {$[-]$} \\
\hline Fraction of channel area blocked by grid & \\
\hline Float & Conditional - if IFESPV $=1$ or 2 \\
\hline$>=0.0$ and $<1.0$ & \\
\hline
\end{tabular}

\begin{tabular}{|l|l|l|c|}
\hline $\mathbf{7 . 3}$ & GLONG (NG) & {$[\mathrm{m}]$} & [in] \\
\hline Grid length & Conditional - if IFESPV $=1$ or 2 \\
\hline Float & \\
\hline$>0.0$ & \\
\hline
\end{tabular}

\begin{tabular}{|l|l|l|l|}
\hline $\mathbf{7 . 3}$ & GPERIM $(\mathrm{NG})$ & {$[\mathrm{m}]$} & {$[\mathrm{in}]$} \\
\hline Grid perimeter & Conditional - if IFESPV $=1$ or 2 \\
\hline Float & \\
\hline$>0.0$ & \\
\hline
\end{tabular}

\begin{tabular}{|l|l|c|c|}
\hline $\mathbf{7 . 3}$ & SPBLOC $(\mathrm{NG})$ & {$[-]$} & {$[-]$} \\
\hline \multicolumn{2}{|l|}{ Ratio of the area blocked by the grid springs to the channel flow area } \\
\hline Float & Conditional - if IFESPV $=1$ or 2 \\
\hline$>=0.0$ and $<1.0$ & \\
\hline
\end{tabular}

\begin{tabular}{|l|l|l|l|}
\hline $\mathbf{7 . 3}$ & TPROBE $(\mathrm{NG})$ & {$[\mathrm{s}]$} & {$[\mathrm{s}]$} \\
\hline \multicolumn{2}{|l|}{ Time scale for grid quench } \\
\hline Float & Conditional - if IFESPV $=1$ or 2 \\
\hline$>0.0$
\end{tabular}




\begin{tabular}{|l|l|c|c|}
\hline $\mathbf{7 . 3}$ & GTHICK $(\mathrm{NG})$ & {$[\mathrm{m}]$} & [in] \\
\hline Thickness of the straps & & \\
\hline Float & Conditional - if IFESPV $=1$ or 2 \\
\hline$>0.0$ & \\
\hline
\end{tabular}

CARD 7.4: NNGL (NG, NN), NN = $1: \mathrm{NGAL}$

\begin{tabular}{|l|l|l|l|}
\hline $\mathbf{7 . 4}$ & NNGL (NG, NN) & {$[-]$} \\
\hline Axial node number of momentum cells containing grid type ING & {[]} \\
\hline Integer & Conditional - if IFESPV =1 or 2 \\
\hline$>0$ and $<=$ number of momentum cells in channel \\
\hline
\end{tabular}

Card 7.5 is read NGCL(NG) times. Up to 6 sets of NGROD, NGSURF may be entered. If the number of rods with surfaces surrounding a grid is less than 6 , enter $(0 ; 0)$ until 6 sets have been entered.

CARD 7.5: NCNGL (NG, M), GMULT (NG, M), NGROD (NG, M, L), NGSURF (NG, M, L); L = 1:6; M = 1:NGCL (NG)

\begin{tabular}{|l|l|l|l|}
\hline $\mathbf{7 . 5}$ & NCNGL (NG, M) & {$[-]$} & {$[-]$} \\
\hline Channel ID number with grid type ING at axial levels NNGL (NG, NN) as specified \\
\hline Integer & Conditional - if IFESPV = 1 or 2 \\
\hline Must be channel defined in model \\
\hline
\end{tabular}

\begin{tabular}{|l|l|l|l|}
\hline $\mathbf{7 . 5}$ & GMULT (NG, M) & {$[-]$} & {$[-]$} \\
\hline Number of grids contained in channel NCNGL (NG, NN) & \\
\hline Integer & Conditional - if IFESPV $=1$ or 2 \\
\hline$>0$ & \\
\hline
\end{tabular}

\begin{tabular}{|l|l|l|l|}
\hline $\mathbf{7 . 5}$ & NGROD (NG, M, L) & {$[-]$} & {$[-]$} \\
\hline $\begin{array}{l}\text { Whole rod number with surface surrounding grid } \\
\text { Maximum of } 6\end{array}$ \\
\hline Integer & Conditional - if IFESPV = 1 or 2 \\
\hline Must be rod index defined in model \\
\hline
\end{tabular}

\begin{tabular}{|c|c|c|c|}
\hline 7.5 & $\operatorname{NGSURF}(\mathrm{NG}, \mathrm{M}, \mathrm{L})$ & {$[-]$} & {$[-]$} \\
\hline \multicolumn{4}{|c|}{$\begin{array}{l}\text { Rod surface index of whole rod NGROD (NG, M, L) surrounding grid ING } \\
\text { Note: The average temperature of all surfaces surrounding grid is used to transport heat between grid } \\
\text { and heater rods. }\end{array}$} \\
\hline Integer & Conditional - if IFE & & \\
\hline
\end{tabular}

Card 7.6 and 7.7 are read NFBS times. 
CARD 7.6: IBS, IFB (IBS), JSFB (IBS), NRFB(IBS), SPOINT(IBS), DSEP(IBS), THROAT(IBS), AFLBLK(IBS), $\operatorname{CDFB}($ IBS $), \operatorname{ABLOCK}($ IBS $) ; \operatorname{IBS}=1: \mathrm{NFBS}$

\begin{tabular}{|c|c|c|c|}
\hline 7.6 & IBS & {$[-]$} & {$[-]$} \\
\hline \multicolumn{4}{|c|}{$\begin{array}{l}\text { Flow blockage index number } \\
\text { (Must be sequential from } 1 \text { to NFBS) }\end{array}$} \\
\hline Integer & \multicolumn{3}{|c|}{ Conditional - if NFBS $>0$} \\
\hline \multicolumn{4}{|l|}{-} \\
\hline 7.6 & $\operatorname{IFB}(\mathrm{IBS})$ & {$[-]$} & {$[-]$} \\
\hline \multicolumn{4}{|c|}{ Channel index number } \\
\hline Integer & Condition & & \\
\hline
\end{tabular}

\begin{tabular}{|l|l|l|}
\hline 7.6 & JSFB (IBS) & {$[-]$} \\
\hline Axial node index number \\
\hline Integer & Conditional - if NFBS $>0$ \\
\hline$>0$ and $<=$ number of momentum levels in channel \\
\hline
\end{tabular}

\begin{tabular}{|l|l|l|}
\hline $\mathbf{7 . 6}$ & NRFB (IBS) & {$[-]$} \\
\hline Number of rods that block this channel & {$[-]$} \\
\hline Integer & Conditional - if NFBS $>0$ \\
\hline$>0$ & \\
\hline
\end{tabular}

\begin{tabular}{|l|l|l|}
\hline $\mathbf{7 . 6}$ & SPOINT (IBS) & {$[\mathrm{m}]$} \\
\hline Axial position of the flow separation point & \\
\hline Float & Conditional - if NFBS $>0$ \\
\hline$>=0.0$ & \\
\hline
\end{tabular}

\begin{tabular}{|c|c|c|c|}
\hline 7.6 & DSEP (IBS) & {$[\mathrm{m}]$} & [in] \\
\hline \multicolumn{4}{|c|}{ Channel diameter at separation point } \\
\hline Float & Conditional - if NFBS $>0$ & & \\
\hline$>0.0$ & & & \\
\hline
\end{tabular}

\begin{tabular}{|c|c|c|c|}
\hline 7.6 & THROAT (IBS) & {$[\mathrm{m}]$} & [in] \\
\hline \multicolumn{4}{|c|}{ Diffuser diamter at exit } \\
\hline Float & \multicolumn{3}{|c|}{ Conditional - if NFBS $>0$} \\
\hline$>0.0$ & & & \\
\hline
\end{tabular}




\begin{tabular}{|l|l|c|c|}
\hline $\mathbf{7 . 6}$ & AFLBLK (IBS) & {$[-]$} & {$[-]$} \\
\hline Area for DBM, (fraction of channel) $/ 4$ & \\
\hline Integer & Conditional - if NFBS $>0$ \\
\hline$>0.0$ & \\
\hline
\end{tabular}

\begin{tabular}{|l|l|l|l|}
\hline $\mathbf{7 . 6}$ & CDFB (IBS) & {$[-]$} & {$[-]$} \\
\hline Presure loss coefficient (Rehme multiplier) & \\
\hline Integer & Conditional - if NFBS $>0$ \\
\hline$>=0.0$ & \\
\hline
\end{tabular}

\begin{tabular}{|l|l|c|c|}
\hline $\mathbf{7 . 6}$ & ABLOCK (IBS) & {$[-]$} & {$[-]$} \\
\hline Blockage area ratio & & \\
\hline Integer & Conditional - if NFBS $>0$ \\
\hline$>=0.0$ and $<1.0$ & \\
\hline
\end{tabular}

CARD 7.7: NRODFB(IBS, N), KRODFB(IBS, N), ANGIHT(IBS, N), ARAIHT (IBS, N); $N=1$ :NRFB

\begin{tabular}{|l|l|l|l|}
\hline $\mathbf{7 . 7}$ & NRODFB (IBS, N) & {$[-]$} & {$[-]$} \\
\hline Index number of rod & \\
\hline Integer & Conditional - if NFBS $>0$ \\
\hline Must be rod index defined in model \\
\hline
\end{tabular}

\begin{tabular}{|l|l|c|c|}
\hline $\mathbf{7 . 7}$ & KRODFB (IBS, N) & {$[-]$} & {$[-]$} \\
\hline Surface number & & \\
\hline Integer & Conditional - if NFBS $>0$ \\
\hline \multicolumn{2}{|l|}{ Must be surface index defined in model } \\
\hline
\end{tabular}

\begin{tabular}{|l|l|l|l|}
\hline $\mathbf{7 . 7}$ & ANGIHT (IBS, N) & [degrees] & [degrees] \\
\hline Angle for impact heat transfer & \\
\hline Float & Conditional - if NFBS $>0$ \\
\hline$>=0.0$ and $<360.0$ & \\
\hline
\end{tabular}

\begin{tabular}{|c|c|c|c|}
\hline 7.7 & ARAIHT (IBS, N) & {$\left[\mathrm{m}^{2}\right]$} & {$\left[\mathrm{in}^{2}\right]$} \\
\hline \multicolumn{4}{|c|}{ Area for impact heat transfer (per rod) } \\
\hline Float & Conditional - if 1 & & \\
\hline$>0.0$ & & & \\
\hline
\end{tabular}

Card 7.8 is read only if IGTEMP $\neq 0$. This option bypasses the original TGRID calculation.

CARD 7.8: TGRID(I), QFGRID(I); I = 1 : IGTEMP 


\begin{tabular}{|l|l|c|c|}
\hline 7.8 & TGRID $(\mathrm{I})$ & {$\left[{ }^{\circ} \mathrm{C}\right]$} & {$\left[{ }^{\circ} \mathrm{F}\right]$} \\
\hline Grid temperature & & \\
\hline Float & Conditional - if IGTEMP $\neq 0$ \\
\hline$>0.0$ & \\
\hline
\end{tabular}

\begin{tabular}{|l|l|c|c|}
\hline 7.8 & QFGRID $(\mathrm{I})$ & {$[\mathrm{m}]$} & {$[\mathrm{in}]$} \\
\hline \multicolumn{2}{|l|}{ Length of the quenched portion of the grid } & \\
\hline Float & Conditional - if IGTEMP $\neq 0$ & \\
\hline$>0.0$ & \\
\hline
\end{tabular}

Card 7.9 is read only if IXFLOW $\neq 0$. This option is for the spacer grid-induced cross-flow effects.

CARD 7.9 VALUE_ANGLE, NSETS_XFLOW, NSETS_SG_MULT

\begin{tabular}{|l|l|l|l|}
\hline $\mathbf{7 . 9}$ & VALUE_ANGLE & [degrees] & [degrees] \\
\hline \multicolumn{3}{|l|}{ Vane angle } & Conditional - if IXFLOW $\neq 0$ \\
\hline Float & \\
\hline$>=0.0$ and $<90.0$ & \\
\hline
\end{tabular}

\begin{tabular}{|l|l|l|l|}
\hline $\mathbf{7 . 9}$ & NSETS_XFLOW & {$[-]$} & {$[-]$} \\
\hline $\begin{array}{l}\text { Number of data sets in input file xflow_data } \\
\text { If IXFLOW }=1 \text { or 3, then NSETS_XFLOW should be }>0 .\end{array}$ \\
\hline Integer & Conditional - if IXFLOW $\neq 0$ \\
\hline$>0$ & \\
\hline
\end{tabular}

\begin{tabular}{|l|l|l|l|}
\hline $\mathbf{7 . 9}$ & NSETS_SG_MULT & {$[-]$} & {$[-]$} \\
\hline $\begin{array}{l}\text { Number of data sets in input file sg_mult_data } \\
\text { If IXFLOW }=2 \text { or } 3, \text { then NSETS_SG_MULT should be }>0 .\end{array}$ \\
\hline Integer & Conditional - if IXFLOW $\neq 0$ \\
\hline$>0$ & \\
\hline
\end{tabular}




\section{Card Group 8}

NOTE: Even if not modeling any conductors, the Card Group number (8) and Card 8.1, specifying zero rods and zero unheated conductors must be entered.

The first line indicates the group number: NGROUP $=8$

CARD 8.1: NRROD, NSROD, NC, NRTAB, NRAD, NLTYP, NSTATE, NXF, NCAN, RADFLG, W3CHF, IHTC, DNBCHK, IKFUEL

\begin{tabular}{|l|l|c|c|}
\hline $\mathbf{8 . 1}$ & NRROD & {$[-]$} & {$[-]$} \\
\hline Number of rods & & \\
\hline Integer & Required & \\
\hline$>=0$ & \\
\hline
\end{tabular}

\begin{tabular}{|l|l|c|c|}
\hline $\mathbf{8 . 1}$ & NSROD & {$[-]$} & {$[-]$} \\
\hline \multicolumn{2}{|l|}{ Number of unheated conductors } & \\
\hline Integer & Required & \\
\hline$>=0$ & \\
\hline
\end{tabular}

\begin{tabular}{|c|c|c|c|}
\hline 8.1 & NC & {$[-]$} & {$[-]$} \\
\hline \multicolumn{4}{|c|}{ Conduction model flag: } \\
\hline \multicolumn{4}{|c|}{0 - No conduction } \\
\hline \multicolumn{4}{|c|}{1 - Radial conduction only } \\
\hline \multicolumn{4}{|c|}{2 - Radial and axial conduction } \\
\hline \multicolumn{4}{|c|}{3 - Radial, axial, and azimuthal conduction } \\
\hline \multicolumn{4}{|c|}{ Note: For unheated conductors, radial conduction is regarded at most. } \\
\hline Integer & Required & & \\
\hline - & & & \\
\hline
\end{tabular}




\begin{tabular}{|c|c|c|c|}
\hline 8.1 & NRTAB & {$[-]$} & {$[-]$} \\
\hline \multicolumn{4}{|c|}{$\begin{array}{l}\text { Number of temperature initialization tables to be read in CARD } 8.6 \text { through CARD 8.9: } \\
\geq 1 \text { - Mandatory }\end{array}$} \\
\hline Integer & Required & & \\
\hline
\end{tabular}

\begin{tabular}{l}
\begin{tabular}{|l|l|l|l|}
\hline $\mathbf{8 . 1}$ & NRAD & {$[-]$} & {$[-]$} \\
\hline $\begin{array}{l}\text { Number of radiation channels: } \\
0 \text { - If no radiation is modeled (if RADFLG }=0)\end{array}$ \\
\hline Integer & Required \\
\hline$>=0$ &
\end{tabular} \\
\hline
\end{tabular}

\begin{tabular}{|c|c|c|c|}
\hline 8.1 & NLTYP & {$[-]$} & {$[-]$} \\
\hline \multicolumn{4}{|c|}{ Number of geometry types for the radiation calculations: } \\
\hline \multicolumn{4}{|c|}{0 - If no radiation is modeled (if $\mathrm{RADFLG}=0$ ) } \\
\hline Integer & Required & & \\
\hline$>=0$ & & & \\
\hline
\end{tabular}

\begin{tabular}{|c|c|c|c|}
\hline 8.1 & NSTATE & {$[-]$} & {$[-]$} \\
\hline \multicolumn{4}{|c|}{ Flag for steady-state calculation of rod temperatures: } \\
\hline \multicolumn{4}{|c|}{0 - Full transient calculation (default) } \\
\hline \multicolumn{4}{|c|}{$>0$ - Steady-state solution of rod temperatures at the beginning of a calculation } \\
\hline Integer & Required & & \\
\hline$>=0$ & & & \\
\hline
\end{tabular}

\begin{tabular}{|c|c|c|c|}
\hline 8.1 & NXF & {$[-]$} & {$[-]$} \\
\hline \multicolumn{4}{|c|}{ Number of time steps between radiation calculations: } \\
\hline \multicolumn{4}{|c|}{1 - Default } \\
\hline Integer & Required & & \\
\hline$>=1$ & & & \\
\hline
\end{tabular}




\begin{tabular}{|c|c|c|c|}
\hline 8.1 & NCAN & {$[-]$} & {$[-]$} \\
\hline \multicolumn{4}{|c|}{$\begin{array}{l}\text { Flag for the Yamanouchi canister quench model (spray cooling after LOCA): } \\
\leq 0 \text { - Off } \\
>0 \text { - On }\end{array}$} \\
\hline Integer & Required & & \\
\hline- & & & \\
\hline
\end{tabular}

\begin{tabular}{|l|l|l|l|}
\hline $\mathbf{8 . 1}$ & RADFLG & {$[-]$} & {$[-]$} \\
\hline Flag for the radiation heat transfer calculations only: & \\
0 - Off \\
$\neq 0$ - On \\
2 - On, excluding fluid \\
\hline Integer & \\
\hline- & Required \\
\hline
\end{tabular}

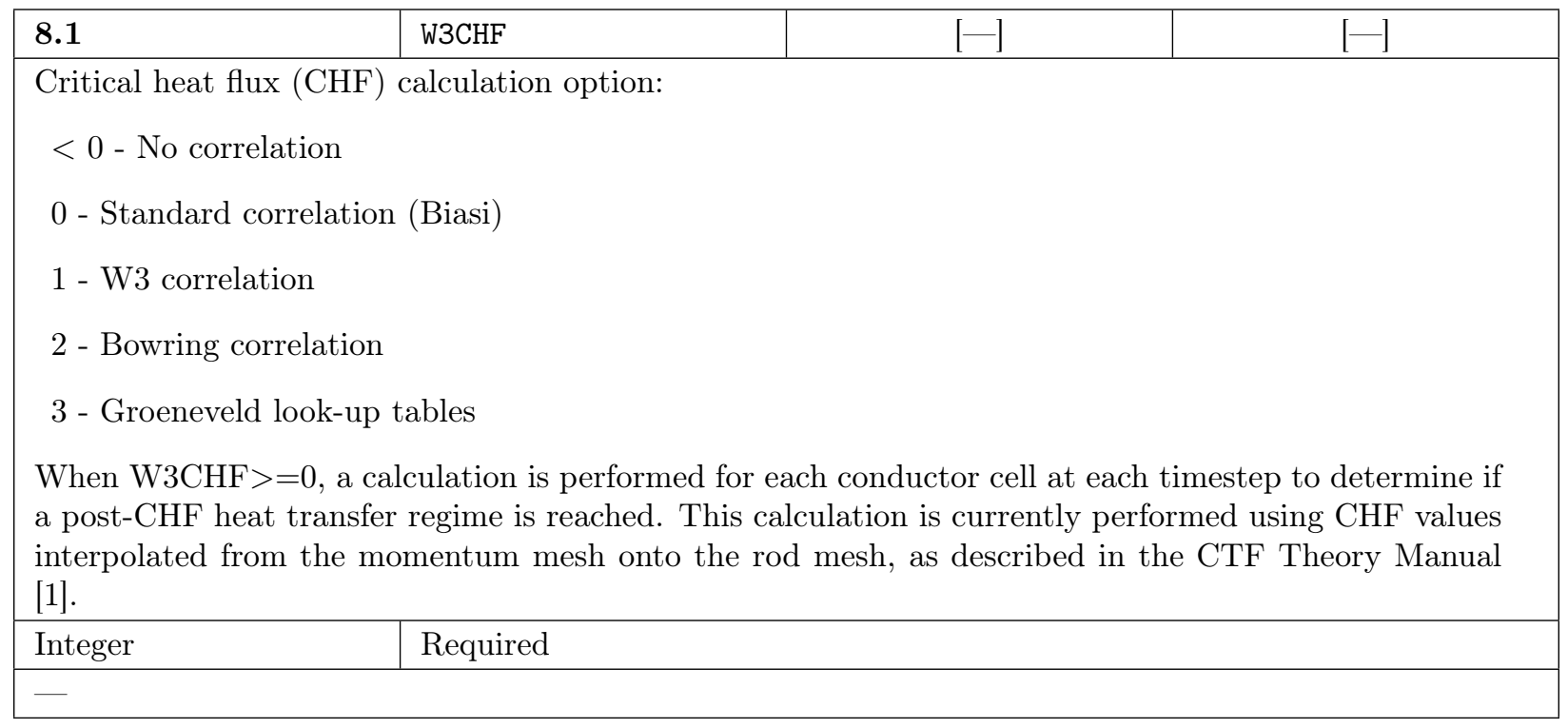




\begin{tabular}{|c|c|c|c|}
\hline 8.1 & IHTC & {$[-]$} & {$[-]$} \\
\hline \multicolumn{4}{|c|}{$\begin{array}{l}\text { Choice for the nucleate boiling model. Whichever is chosen will be used in both sub-cooled and } \\
\text { saturated boiling regions: } \\
0 \text { - Chen } \\
1 \text { - Thom } \\
2 \text { - Gorenflo }\end{array}$} \\
\hline Integer & Required & & \\
\hline - & & & \\
\hline
\end{tabular}

\begin{tabular}{|c|c|c|c|}
\hline 8.1 & DNBCHK & {$[-]$} & {$[-]$} \\
\hline \multicolumn{4}{|c|}{ DNB check option: } \\
\hline \multicolumn{4}{|c|}{-1 - Do not check DNB data } \\
\hline \multicolumn{4}{|c|}{0 - Standard correlation (Biasi) } \\
\hline \multicolumn{4}{|c|}{1 - W3 correlation } \\
\hline \multicolumn{4}{|c|}{2 - Bowring correlation } \\
\hline \multicolumn{4}{|c|}{3 - Groeneveld look-up tables } \\
\hline \multirow{8}{*}{\multicolumn{4}{|c|}{$\begin{array}{l}\text { Has no effect when } W 3 C H F>=0 \text {. This option is used if the W3CHF model is disabled. It calculates } \\
\text { DNB data in the model prior to writing edits. When W3CHF is }-1 \text {, it is not possible for the code to go } \\
\text { into post-CHF heat transfer regimes; however, it is still useful to do a calculation of DNB data in the } \\
\text { model in order to inform the user of the DNB margin. Because this check is only done prior to writing } \\
\text { edits, rather than every iteration, the simulation cost is greatly reduced. This option is disabled when } \\
\text { W3CHF }>=0 \text { because the DNB data is already being calculated using the correlation selected with } \\
\text { W3CHF for each iteration. If either W3CHF }>=0 \text { or DNBCHK }>=0 \text {, a special post-processing CHF } \\
\text { calculation will be performed using CHF values interpolated from the scalar mesh onto the rod mesh, } \\
\text { as described in the CTF Theory Manual [1]. }\end{array}$}} \\
\hline & & & \\
\hline & & & \\
\hline & & & \\
\hline & & & \\
\hline & & & \\
\hline & & & \\
\hline & & & \\
\hline Integer & Required & & \\
\hline \multicolumn{4}{|l|}{-} \\
\hline
\end{tabular}




\begin{tabular}{|c|c|c|c|}
\hline 8.1 & IKFUEL & {$[-]$} & {$[-]$} \\
\hline \multicolumn{4}{|c|}{$\begin{array}{l}\text { This option is used to set the pellet thermal conductivity model. Negative numbers will apply one of } \\
\text { the built-in burnup-dependent models. The selected model will be applied in all fuel rods in the model } \\
\text { and initial burnup and gadolinia content in the rod will be zero. These options are intended to be } \\
\text { used while CTF is coupled to an external code that will set burnup and gadolina. Option zero uses the } \\
\text { default MATPRO- } 11 \text { model, which has no burnup depedence. Option } 1 \text { means that Card } 8.14 \text { will be } \\
\text { read in later to specify the local burnup and gadolina distribution in each rod in the model. This also } \\
\text { allows different models to be used for different rods and axial locations. The specific model to be used } \\
\text { will be set on Card } 8.14 .2 \text { if using option } 1 \text {. }\end{array}$} \\
\hline \multicolumn{4}{|c|}{-6 - Modified NFIR model for $\mathrm{UO}_{2}$} \\
\hline \multicolumn{4}{|c|}{-5 - Amaya correlation (MOX fuel) } \\
\hline \multicolumn{4}{|c|}{-4 - Halden correlation (MOX fuel) } \\
\hline \multicolumn{4}{|c|}{$-3-$ Duriez/Modified NFI correlation (MOX fuel) } \\
\hline \multicolumn{4}{|c|}{-2 - Halden correlation $\left(\mathrm{UO}_{2}\right.$ fuel $)$} \\
\hline \multicolumn{4}{|c|}{-1 - Modified NFI correlation $\left(\mathrm{UO}_{2}\right.$ fuel $)$} \\
\hline \multicolumn{4}{|c|}{0 - Default model (currently -6) } \\
\hline \multicolumn{4}{|c|}{$k_{u o 2}=C\left\{\max \left(\frac{2335}{464+T}, 1.1038\right)+7.027 \cdot 10^{-3} \exp \left(1.867 \cdot 10^{-3} T\right)\right.$} \\
\hline \multicolumn{4}{|c|}{1 - Read Card 8.14. } \\
\hline Integer & Required & & \\
\hline
\end{tabular}

\subsection{Rod Geometry Data}

Card 8.2, Card 8.3, and Card 8.4 are read NRROD times.

CARD 8.2: N, $\operatorname{IFTYP}(\mathrm{N}), \operatorname{IAXP}(\mathrm{N}), \operatorname{NRENODE}(\mathrm{N}), \operatorname{DAXMiN}(\mathrm{N}), \operatorname{RMULT}(\mathrm{N}), \operatorname{HGAP}(\mathrm{N}), \operatorname{ISECR}(\mathrm{N}), \operatorname{HTAMB}(\mathrm{N})$, TAMB $(N)$, SYMROD $(N)$, IHTCMAP, ITKEMAP, NSUBSECT, NSUBAX

\begin{tabular}{|c|c|c|c|}
\hline 8.2 & $\mathrm{~N}$ & {$[-]$} & {$[-]$} \\
\hline \multicolumn{4}{|c|}{$\begin{array}{l}\text { Index of rod } \\
\text { Note: Rod indices must be entered sequentially, from } 1 \text { to NRROD. Skipping numbers is not permitted } \\
\text { Note 2: A negative value of the rod index is used to flag a rod with inside connections to a fluid channel } \\
\text { and cause Card } 8.4 \text { to be read. }\end{array}$} \\
\hline Integer & Required & & \\
\hline- & & & \\
\hline
\end{tabular}




\begin{tabular}{|l|l|l|l|}
\hline $\mathbf{8 . 2}$ & IFTYP (N) & {$[-]$} & {$[-]$} \\
\hline $\begin{array}{l}\text { Geometry type identification number } \\
\text { (Refers to CARD GROUP 9 for geometry type input data) }\end{array}$ \\
\hline Integer & Required \\
\hline-
\end{tabular}

\begin{tabular}{|c|c|c|c|}
\hline 8.2 & $\operatorname{IAXP}(\mathrm{N})$ & {$[-]$} & {$[-]$} \\
\hline \multicolumn{4}{|c|}{$\begin{array}{l}\text { Axial power profile table identification number } \\
\text { (Refers to CARD } 11.3 \text { and } 11.4 \text { for axial power profile input data). If left blank, a uniform axial power } \\
\text { distribution will be assumed. }\end{array}$} \\
\hline Integer & Required & & \\
\hline- & & & \\
\hline
\end{tabular}

\begin{tabular}{|l|l|l|}
\hline $\mathbf{8 . 2}$ & $\operatorname{NRENODE}(\mathrm{N})$ & {$[-]$} \\
\hline Re-noding flag for heat transfer solution for rod $\mathrm{N}$ : \\
0 - No fine-mesh re-noding \\
$>0$ - Re-noding every NRENODE (N) time steps \\
$<0$ - Re-noding every NRENODE (N) time steps, based on inside surface temperatures \\
\hline Integer & Required \\
\hline
\end{tabular}

\begin{tabular}{|l|l|c|c|}
\hline $\mathbf{8 . 2}$ & DAXMIN $(\mathrm{N})$ & {$[\mathrm{m}]$} & [in] \\
\hline $\begin{array}{l}\text { Minimum axial node size } \\
\text { This is only used if NRENODE } \neq 0 .\end{array}$ & & \\
\hline Float & Required & \\
\hline$>0.0$ &
\end{tabular}

\begin{tabular}{|l|l|l|}
\hline $\mathbf{8 . 2}$ & RMULT (N) & {$[-]$} \\
\hline $\begin{array}{l}\text { Rod multiplication factor (number of rods modeled by the single rod N) } \\
\text { Should be }>=1.0 . \text { If modeling a partial rod, use the SYMROD parameter. }\end{array}$ \\
\hline Float & Required \\
\hline-
\end{tabular}




\begin{tabular}{|c|c|c|c|}
\hline 8.2 & $\operatorname{HGAP}(\mathrm{N})$ & {$\left[\frac{W}{m^{2} K}\right]$} & {$\left[\frac{B T U}{h r f t^{2} \circ F}\right]$} \\
\hline \multicolumn{4}{|c|}{ Constant gap conductance: } \\
\hline \multicolumn{4}{|c|}{0 - If rod $\mathrm{N}$ does not model a nuclear fuel rod } \\
\hline \multicolumn{4}{|c|}{$\begin{array}{l}\text { This parameter is used only for nuclear fuel rods that do not have the dynamic gap conductance model } \\
\text { specified by their geometry type }\end{array}$} \\
\hline Float & Required & & \\
\hline$>0.0$ & & & \\
\hline
\end{tabular}

\begin{tabular}{|l|l|c|c|}
\hline $\mathbf{8 . 2}$ & ISECR $(\mathrm{N})$ & {$[-]$} & {$[-]$} \\
\hline \multicolumn{2}{|l|}{ Total number of sections containing rod $\mathrm{N}$} & \\
\hline Integer & Required & \\
\hline$>0$ &
\end{tabular}

\begin{tabular}{|c|c|c|c|}
\hline 8.2 & $\operatorname{HTAMB}(\mathrm{N})$ & {$\left[\frac{W}{m^{2} K}\right]$} & {$\left[\frac{B T U}{h r f t^{2} \circ F}\right]$} \\
\hline \multicolumn{4}{|c|}{$\begin{array}{l}\text { Heat transfer coefficient for heat loss to the ambience from a surface not connected to a coolant channel } \\
\text { Note: This option is normally not related to the real fuel rod simulation. It is used in some simulations } \\
\text { of experiments when the user wants to account for the heat loss from a non water-cooled rod to the } \\
\text { ambience or to the medium inside the tube. }\end{array}$} \\
\hline Float & Required & & \\
\hline
\end{tabular}

\begin{tabular}{|l|l|c|c|}
\hline $\mathbf{8 . 2}$ & TAMB $(\mathrm{N})$ & {$\left[{ }^{\circ} \mathrm{C}\right]$} & {$\left[{ }^{\circ} \mathrm{F}\right]$} \\
\hline \multicolumn{2}{|l|}{ Sink temperature for heat loss to ambience } & \\
\hline Float & Required & \\
\hline$>0.0$ &
\end{tabular}

\begin{tabular}{|c|c|c|c|}
\hline 8.2 & SYMROD (N) & {$[-]$} & {$[-]$} \\
\hline \multicolumn{4}{|c|}{$\begin{array}{l}\text { Set to } 1.0 \text { if this is a whole rod or a fraction (e.g., } 0.25,0.5 \text {, etc.) if it is a partial rod (meaning that } \\
\text { a symmetry line runs through the rod. For example, a quarter symmetry model with a symmetry line } \\
\text { running through the rods will have a quarter rod (SYMROD }=0.25) \text { and half rods }(\mathrm{SYMROD}=0.5) \text {. } \\
\text { Can only be used if PPV=2 (see Card } 1.1 \text {. }\end{array}$} \\
\hline Float & Required if $\mathrm{PPV}=2$ & & \\
\hline- & & & \\
\hline
\end{tabular}

Cards 8.3 and 8.4 are read in pairs ISECR(N) times. Note that a rod can start in any axial section in the model; it does not need to start in Section 1. In the following documentation for Cards 8.3 and 8.4, IS represents the section index; it is used only to show that an entry should be made for each section. There is no need to state the actual sections in which the rod exists. The user only specifies to which channel the rod connects. 


\begin{tabular}{l|l|c|}
\hline $\mathbf{8 . 2}$ & IHTCMAP & {$[-]$} \\
\hline $\begin{array}{l}\text { Only required if PPV }>=6 . \text { Integer index that identifies an HTC multiplier map that will be used by } \\
\text { this rod to reconstruct high-fidelity heat transfer effects. Only used for coupled crud solutions. Set to } \\
0 \text { for no HTC multiplier map. }\end{array}$ \\
\hline Integer & Required if PPV $=6$ \\
\hline-
\end{tabular}

\begin{tabular}{|c|c|c|c|}
\hline 8.2 & ITKEMAP & {$[-]$} & {$[-]$} \\
\hline \multicolumn{4}{|c|}{$\begin{array}{l}\text { Only required if } \mathrm{PPV}>=6 \text {. Integer index that identifies a TKE multiplier map that will be used by } \\
\text { this rod to reconstruct high-fidelity wall shear effects. Only used for coupled crud solutions. Set to } 0 \\
\text { for no TKE multiplier map. }\end{array}$} \\
\hline Integer & Required & & \\
\hline
\end{tabular}

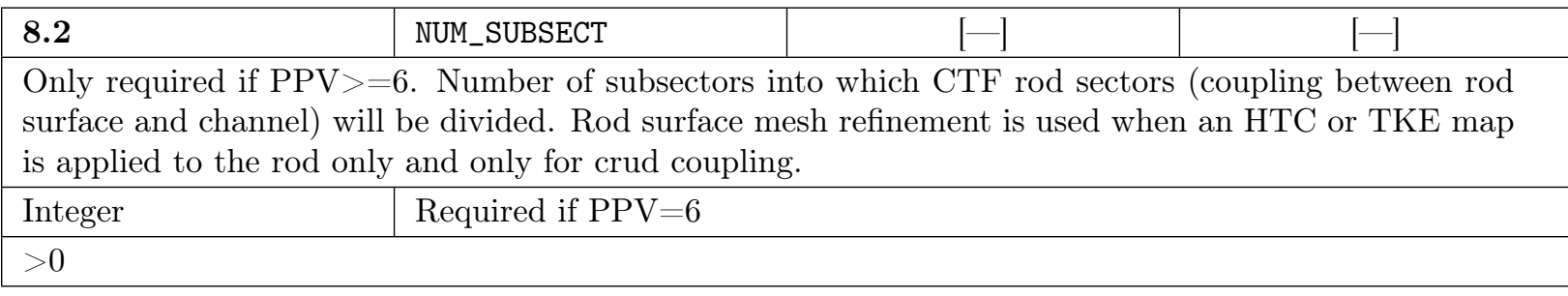

\begin{tabular}{|c|c|c|c|}
\hline 8.2 & NUM_SUBAX & {$[-]$} & {$[-]$} \\
\hline \multicolumn{4}{|c|}{$\begin{array}{l}\text { Only required if PPV }>=6 \text {. Number of sublevels into which CTF rod levels will be divided. Rod } \\
\text { surface mesh refinement is used when an HTC or TKE map is applied to the rod only and only for } \\
\text { crud coupling. }\end{array}$} \\
\hline Integer & Required if & & \\
\hline$>0$ & & & \\
\hline
\end{tabular}

Cards 8.3 and 8.4 are read in pairs ISECR(N) times. Note that a rod can start in any axial section in the model; it does not need to start in Section 1. In the following documentation for Cards 8.3 and 8.4, IS represents the section index; it is used only to show that an entry should be made for each section. There is no need to state the actual sections in which the rod exists. The user only specifies to which channel the rod connects.

CARD 8.3: $\operatorname{NSCHC}(I S, K), \operatorname{PIE}(\mathrm{N}, \mathrm{K}) ; \mathrm{K}=1: 8$

Up to 8 sets of (NSCHC, PIE) may be entered. If the rod $\mathrm{N}$ is thermally connected to fewer than 8 channels, enter $(0 ; 0.0)$ until 8 sets of (NSCHC, PIE) have been entered.

\begin{tabular}{|l|l|c|c|}
\hline $\mathbf{8 . 3}$ & NSCHC (IS, K) & {$[-]$} & {$[-]$} \\
\hline Channel number with thermal connections to rod N & \\
\hline Integer & Required \\
\hline Must be channel index defined in model \\
\hline
\end{tabular}




\begin{tabular}{|c|c|c|c|}
\hline 8.3 & $\mathrm{PIE}(\mathrm{N}, \mathrm{K})$ & {$[-]$} & {$[-]$} \\
\hline \multicolumn{4}{|c|}{ Azimuthal fraction of rod $\mathrm{N}$ thermally connected to channel NSCHC $(\mathrm{K})$} \\
\hline (Integer, Float) & Required & & \\
\hline$>0.0$ and $<=1.0$ & & & \\
\hline
\end{tabular}

Up to 8 sets of (NSCHC,PIE) may be entered. If the rod $\mathrm{N}$ is thermally connected to fewer than 8 channels, enter $(0 ; 0.0)$ until 8 sets of (NSCHC,PIE) have been entered.

Note: If a rod has internal fluid connections, as indicated by a negative value of the rod index $N$, then only zeros should be entered for channel numbers on the outside of the rod. This version does not support fluid connections on both sides of a heater rod.

Card 8.4 is read only if an inside surface for rod $\mathrm{N}$ exists, i.e. if $\mathrm{NSCHC}($ IS, 1$)<0$.

CARD 8.4: NISCHC(N, IS, $K) ; K=1: 8$

\begin{tabular}{|l|l|c|c|}
\hline $\mathbf{8 . 4}$ & NISCHC (N, IS, K) & {$[-]$} & {$[-]$} \\
\hline \multicolumn{2}{|l|}{ Negative of channel number connected to the inside of (N, IS, K) azimuthal section K of rod N } \\
\hline Integer & Not Operational - Required \\
\hline \multicolumn{2}{|l|}{ Must be channel defined in model (negative of index) } \\
\hline
\end{tabular}

\subsection{Unheated Conductor Data}

Card 8.5 is read NSROD times for all unheated conductors (also called âĂIJheat slabsâ̆ $\mathbf{I}$ ).

CARD 8.5: $N, \operatorname{ISTYPE}(\mathrm{N}), \operatorname{HPERIM}(\mathrm{N}), \operatorname{HPERIMI}(\mathrm{N}), \operatorname{RMULS}(\mathrm{N}), \mathrm{NOSLCH}, \operatorname{NSLCHC}, \operatorname{HTAMBS}(\mathrm{N}), \operatorname{TAMBS}(\mathrm{N})$

\begin{tabular}{|c|c|c|c|}
\hline 8.5 & $\mathrm{~N}$ & {$[-]$} & {$[-]$} \\
\hline \multicolumn{4}{|c|}{$\begin{array}{l}\text { Index of unheated conductor } \\
\text { Note: Unheated conductor indices must be entered sequentially, from } 1 \text { to NSROD. Skipping numbers is } \\
\text { not permitted. }\end{array}$} \\
\hline Integer & Required & & \\
\hline- & & & \\
\hline
\end{tabular}

\begin{tabular}{|l|l|l|l|}
\hline $\mathbf{8 . 5}$ & ISTYP (N) & {$[-]$} & {$[-]$} \\
\hline $\begin{array}{l}\text { Geometry type identification number } \\
\text { (Refer to CARD GROUP 9 for geometry type input data.) }\end{array}$ \\
\hline Integer & Required \\
\hline- & \\
\hline
\end{tabular}




\begin{tabular}{|l|l|c|}
\hline $\mathbf{8 . 5}$ & $\operatorname{HPERIM}(\mathrm{N})$ & {$[-]$} \\
\hline $\begin{array}{l}\text { Wetted perimeter on outside surface of unheated conductor } \mathrm{N} \\
\text { (Perimeter which is applied to calculate heat transfer from the adjacent subchannel NOSLCH to the } \\
\text { unheated conductor } \mathrm{N})\end{array}$ \\
\\
$\begin{array}{ll}0.0 \text { - If no subchannels are connected to outside surface (NOSLCH }=0) \\
\text { Float }\end{array}$ \\
\hline$>=0.0$ & Required \\
\hline
\end{tabular}

\begin{tabular}{|c|c|c|c|}
\hline 8.5 & HPERIMI (N) & {$[-]$} & {$[-]$} \\
\hline \multicolumn{4}{|c|}{$\begin{array}{l}\text { Wetted perimeter on inside surface of unheated conductor } \mathrm{N} \\
\text { (Perimeter which is applied to calculate heat transfer from the adjacent subchannel NSLCHC to the } \\
\text { unheated conductor } \mathrm{N} \text { ) }\end{array}$} \\
\hline \multicolumn{4}{|c|}{0.0 - If no subchannels are connected to inside surface (NSLCHC $=0)$} \\
\hline Float & Required & & \\
\hline$>=0.0$ & & & \\
\hline
\end{tabular}

\begin{tabular}{|c|c|c|c|}
\hline 8.5 & RMULS (N) & {$[-]$} & {$[-]$} \\
\hline \multicolumn{4}{|c|}{$\begin{array}{l}\text { Unheated conductor multiplication factor (number of unheated conductors modeled by the single un- } \\
\text { heated conductor N) } \\
\text { This number can contain fractional parts. }\end{array}$} \\
\hline Float & Required & & \\
\hline$>0.0$ & & & \\
\hline
\end{tabular}

\begin{tabular}{|c|c|c|c|}
\hline 8.5 & NOSLCH & {$[-]$} & {$[-]$} \\
\hline \multicolumn{4}{|c|}{ Channel number adjacent to outside surface of unheated conductor $\mathrm{N}$} \\
\hline \multicolumn{4}{|c|}{0 - If no subchannels are connected to outside surface } \\
\hline Integer & Requirec & & \\
\hline
\end{tabular}

\begin{tabular}{l}
\begin{tabular}{|l|l|l|}
\hline $\mathbf{8 . 5}$ & NSLCHC & {$[-]$} \\
\hline Channel number adjacent to inside surface of unheated conductor N \\
0 - If no subchannels are connected to inside surface
\end{tabular} \\
\hline \multicolumn{2}{|l|}{ Integer } & Required \\
\hline \multicolumn{2}{|l|}{ Must be channel index defined in model } \\
\hline
\end{tabular}




\begin{tabular}{|c|c|c|c|}
\hline 8.5 & $\operatorname{HTAMBS}(\mathrm{N})$ & {$\left[\frac{W}{m^{2} K}\right]$} & {$\left[\frac{B T U}{h r f t^{2} \circ F}\right]$} \\
\hline \multicolumn{4}{|c|}{$\begin{array}{l}\text { Heat transfer coefficient for heat loss to the ambience } \\
\text { This is used in some simulations of experiments when the user wants to account for the heat loss } \\
\text { from the unheated conductor surfaces (tubes, walls) which are not connected to a coolant channel (not } \\
\text { water-cooled) to the ambience (outside of the bundle, inside a water channel, inside a guide tube). }\end{array}$} \\
\hline Float & Required & & \\
\hline$>0.0$ & & & \\
\hline
\end{tabular}

\begin{tabular}{|l|l|c|c|}
\hline $\mathbf{8 . 5}$ & TAMBS $(\mathrm{N})$ & {$\left[{ }^{\circ} \mathrm{C}\right]$} & {$\left[{ }^{\circ} \mathrm{F}\right]$} \\
\hline \multicolumn{2}{|l|}{ Sink temperature for heat loss to the ambience } & \\
\hline Float & Required & \\
\hline$>0.0$
\end{tabular}

\subsection{Rod Temperature Initialization Tables}

Cards 8.6 through 8.9 are read to specify which temperature tables apply to which rods and unheated conductors. The sequence is repeated NRTAB times, and all rods and conductors must be accounted for.

CARD 8.6: I, NRT1, NST1, NRAX1

\begin{tabular}{|l|l|c|c|}
\hline $\mathbf{8 . 6}$ & I & {$[-]$} & {$[-]$} \\
\hline Identification number of temperature table & & \\
\hline Integer & Required & \\
\hline$>0$ & \\
\hline
\end{tabular}

\begin{tabular}{|l|l|c|c|}
\hline $\mathbf{8 . 6}$ & NRT1 & {$[-]$} & {$[-]$} \\
\hline Number of rods using table I & & \\
\hline Integer & Required & \\
\hline$>=0$ & \\
\hline
\end{tabular}

\begin{tabular}{|l|l|c|c|}
\hline $\mathbf{8 . 6}$ & NST1 & {$[-]$} & {$[-]$} \\
\hline \multicolumn{2}{|l|}{ Number of unheated conductors using table I } & \\
\hline Integer & Required \\
\hline$>=0$ & \\
\hline
\end{tabular}

\begin{tabular}{|l|l|c|c|}
\hline $\mathbf{8 . 6}$ & NRAX1 & {$[-]$} & {$[-]$} \\
\hline Number of pairs of elements in table I & & \\
\hline Integer & Required & \\
\hline$>0$ & \\
\hline
\end{tabular}


Card 8.7 is read only if NRT1 $>0$

CARD 8.7: $\operatorname{IRTAB}(I, L) ; L=1: \operatorname{NRT} 1$

\begin{tabular}{l}
\hline $\mathbf{8 . 7} \quad \operatorname{IRTAB}(\mathrm{I}, \mathrm{L})$ \\
\hline Identification numbers of rods using table I for temperature initialization \\
Enter the negative of the rod identification number if the temperature boundary is to be applied to the \\
inside surface of the rod. \\
Note: The steady-state conduction equation is solved for these rods using the temperatures from table I \\
as a boundary condition on the rod surface. \\
\hline Integer \\
Must be rod indices defined in model \\
\hline
\end{tabular}

Card 8.8 is read only if NST1 $>0$

CARD 8.8: $\operatorname{ISTAB}(I, L) ; \mathrm{L}=1: \mathrm{NST} 1$

\begin{tabular}{|l|l|l|}
\hline $\mathbf{8 . 8}$ & $\operatorname{ISTAB}(\mathrm{I}, \mathrm{L})$ & {$[-]$} \\
\hline Identification numbers of unheated conductors using table I for temperature initialization \\
Note: A flat radial temperature profile is assumed initially in unheated conductors. \\
\hline Integer $\quad$ Conditional - if NST1 >0 \\
\hline Must be unheated conductor indices defined in model
\end{tabular}

CARD 8.9: $\operatorname{AXIALT}(I, L), \operatorname{TRINIT}(I, L) ; L=1: \operatorname{NRAX} 1$

\begin{tabular}{|l|l|c|c|}
\hline $\mathbf{8 . 9}$ & $\operatorname{AXIALT}(\mathrm{I}, \mathrm{L})$ & {$[\mathrm{m}]$} & {$[\mathrm{in}]$} \\
\hline Vertical location & Required & \\
\hline Float & \\
\hline$>=0.0$
\end{tabular}

\begin{tabular}{|l|l|l|}
\hline $\mathbf{8 . 9}$ & $\operatorname{TRINIT}(\mathrm{I}, \mathrm{L})$ & {$\left[{ }^{\circ} \mathrm{C}\right]$} \\
\hline Temperature to be applied at $\operatorname{AXIALT}(\mathrm{I}, \mathrm{L})$ & \\
Note: The vertical locations of the bottom and top of each rod or unheated conductor using table I must \\
be contained within the range $\operatorname{AXIALT}(I, 1)$ to $\operatorname{AXIALT}(I$, NRAX1). \\
\hline Float & Required \\
\hline$>0.0$
\end{tabular}

\subsection{Radiation Initialization Tables}

Card 8.10 through Card 8.11.5 are read in to specify orientation and which location type tables apply to which fluid channels, rods, and unheated conductors. 
Channel Orientation and Location Type Card: 8.10

Card 8.10 is read NRAD times, if NRAD $>0$

CARD 8.10: IRAD, NSIDR (IRAD), LOCATE (IRAD), NRRAD (IRAD), NSYMF(IRAD), MLTF(1, IRAD), MLTF(2, IRAD), $\operatorname{MLTF}(3, \operatorname{IRAD}), \operatorname{MLTF}(4, \operatorname{IRAD}), \operatorname{VDMLT}($ IRAD)

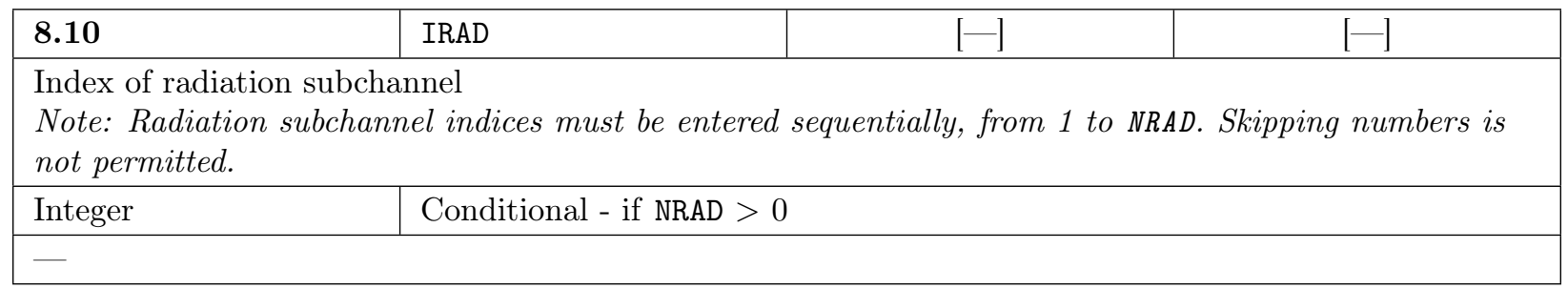

\begin{tabular}{|l|l|l|}
\hline $\mathbf{8 . 1 0}$ & NSIDR (IRAD) & {$[-]$} \\
\hline Index of fluid subchannel & which contains radiation subchannel IRAD \\
\hline Integer & Conditional - if NRAD >0 \\
\hline Must be channel index defined in model \\
\hline
\end{tabular}

\begin{tabular}{|l|l|l|}
\hline $\mathbf{8 . 1 0}$ & LOCATE(IRAD) & {$[-]$} \\
\hline Location type for radiation channel IRAD: & {$[-]$} \\
$<0$ - Contains no unheated conductors \\
$>0$ - Has both rods and unheated conductors \\
\hline Integer & Conditional - if NRAD $>0$ \\
\hline-
\end{tabular}

\begin{tabular}{|l|l|l|}
\hline $\mathbf{8 . 1 0}$ & NRRAD (IRAD) & {$[-]$} \\
\hline Number of contributing radiation surfaces for: & {$[-]$} \\
20 - Location types 1 and 10 & \\
19 - Location type 2 \\
18 - Location type 3 \\
14 - Location type 12 \\
13 - Location type 4 \\
12 - Location types $5,13,14,15,16$, and 17 \\
8 - Location type 6 \\
\hline Integer \\
\hline - Conditional - if NRAD >0 \\
\hline
\end{tabular}




\begin{tabular}{|c|c|c|c|}
\hline 8.10 & NSYMF & {$[-]$} & {$[-]$} \\
\hline \multicolumn{4}{|c|}{ Flag for fluid channel or rod lumping: } \\
\hline \multicolumn{4}{|c|}{0 - No lumping } \\
\hline \multicolumn{4}{|c|}{1 - Lumped fluid channels } \\
\hline Integer & Condit & & \\
\hline - & & & \\
\hline
\end{tabular}

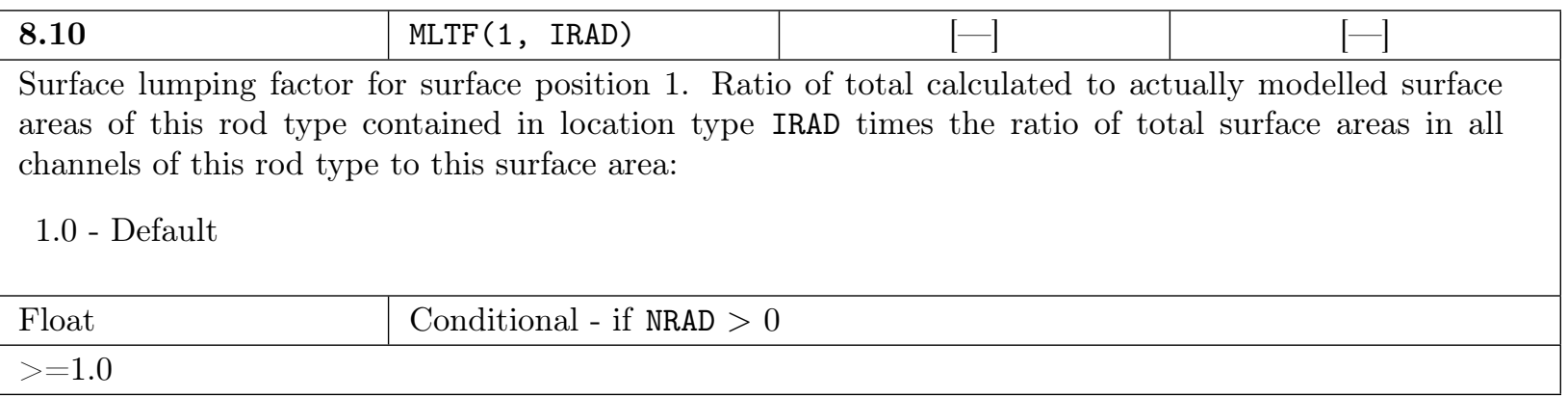

\begin{tabular}{|c|c|c|c|}
\hline 8.10 & $\operatorname{MLTF}(2$, IRAD $)$ & {$[-]$} & {$[-]$} \\
\hline \multicolumn{4}{|c|}{$\begin{array}{l}\text { Surface lumping factor for surface position 2. Ratio of total calculated to actually modelled surface } \\
\text { areas of this rod type contained in location type IRAD times the ratio of total surface areas in all } \\
\text { channels of this rod type to this surface area: }\end{array}$} \\
\hline \multicolumn{4}{|c|}{1.0 - Default } \\
\hline Float & Conditional - if & & \\
\hline \multicolumn{4}{|c|}{$>=1.0$} \\
\hline
\end{tabular}

\begin{tabular}{|c|c|c|c|}
\hline 8.10 & $\operatorname{MLTF}(3, \operatorname{IRAD})$ & {$[-]$} & {$[-]$} \\
\hline \multicolumn{4}{|c|}{$\begin{array}{l}\text { Surface lumping factor for surface position 3. Ratio of total calculated to actually modelled surface } \\
\text { areas of this rod type contained in location type IRAD times the ratio of total surface areas in all } \\
\text { channels of this rod type to this surface area: }\end{array}$} \\
\hline \multicolumn{4}{|c|}{1.0 - Default } \\
\hline Float & Conditional - if & & \\
\hline \multicolumn{4}{|c|}{$>=1.0$} \\
\hline
\end{tabular}




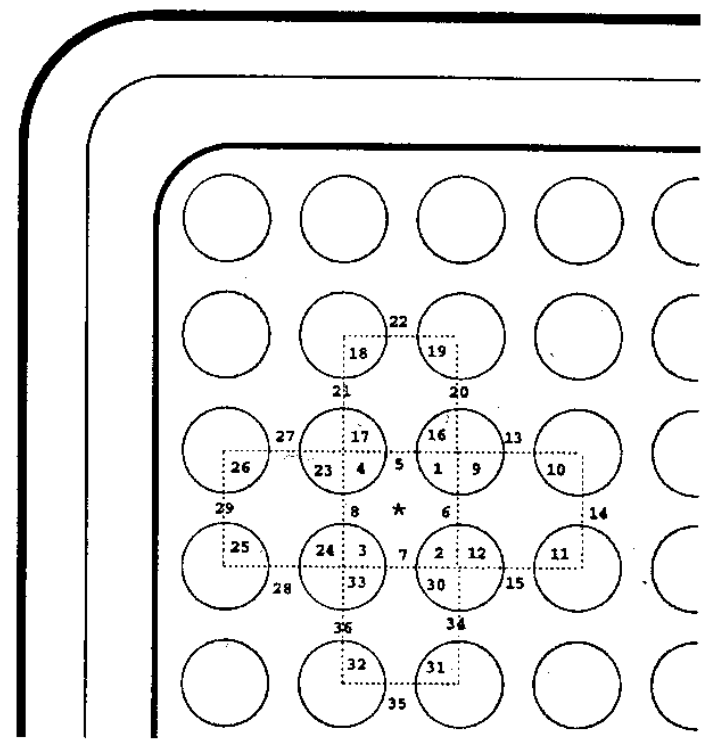

Figure 10.1: Radiation Geometry Type 1

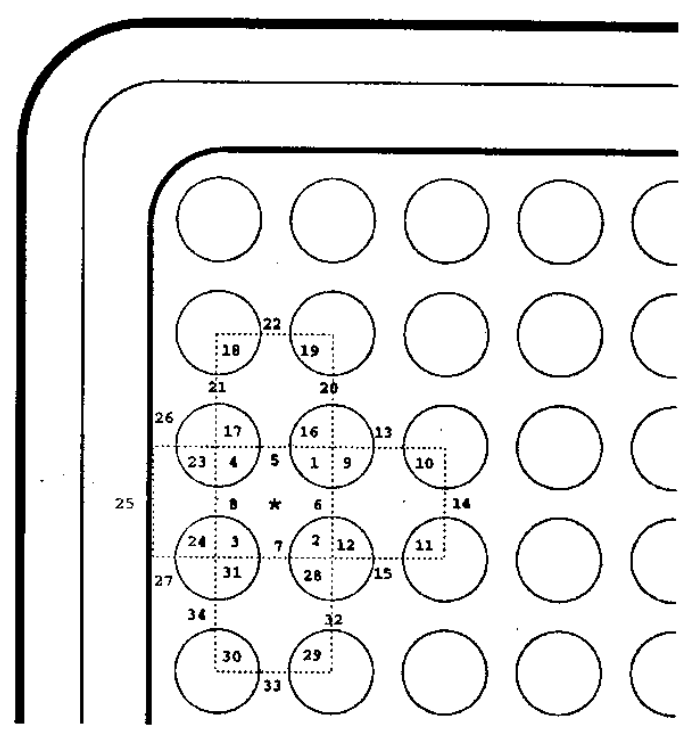

Figure 10.2: Radiation Geometry Type 2

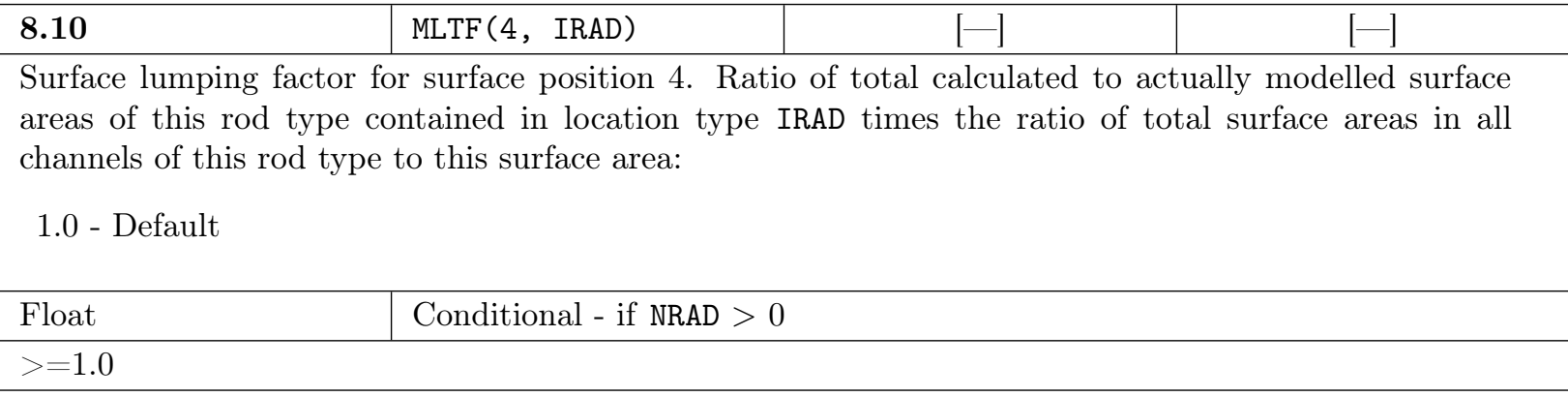

\begin{tabular}{|c|c|c|c|}
\hline 8.10 & VDMLT (IRAD) & {$[-]$} & {$[-]$} \\
\hline \multicolumn{4}{|c|}{$\begin{array}{l}\text { Vapor/droplet multiplication factor. Total number of radiation channels being modeled by this location } \\
\text { type: }\end{array}$} \\
\hline \multicolumn{4}{|c|}{1.0 - Default } \\
\hline Float & \multicolumn{3}{|c|}{ Conditional - if NRAD $>0$} \\
\hline \multicolumn{4}{|l|}{$>=1.0$} \\
\hline
\end{tabular}

\subsection{Radiation Channel Orientation Array}

Repeat Card 8.10.1 and Card 8.10.2 until all radiation channels have been entered. 


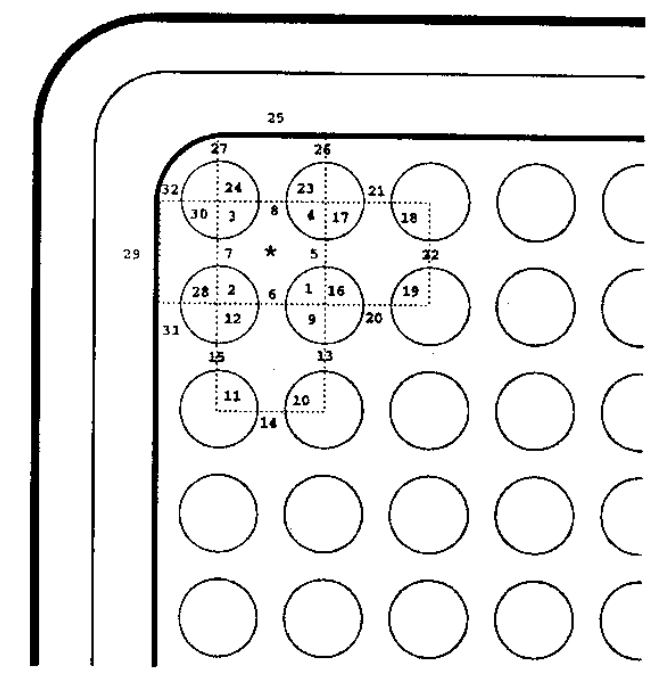

Figure 10.3: Radiation Geometry Type 3

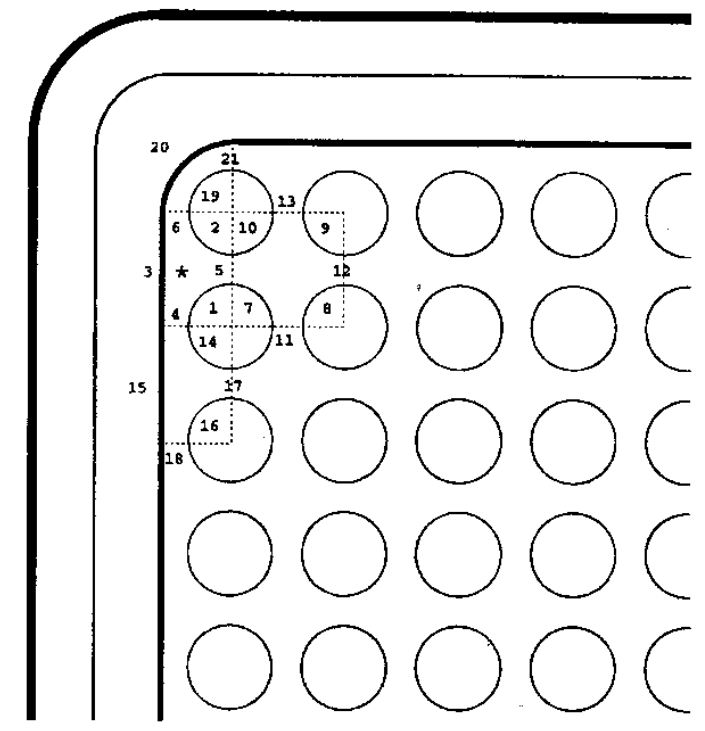

Figure 10.5: Radiation Geometry Type 5

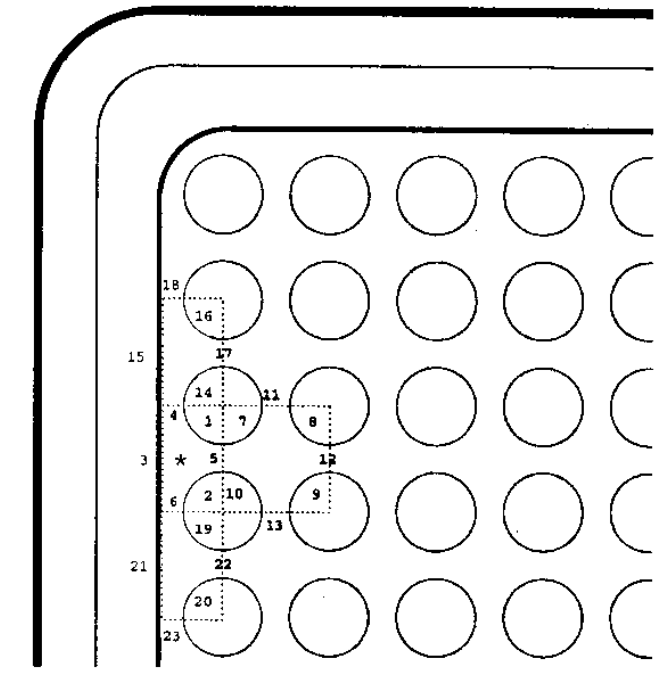

Figure 10.4: Radiation Geometry Type 4

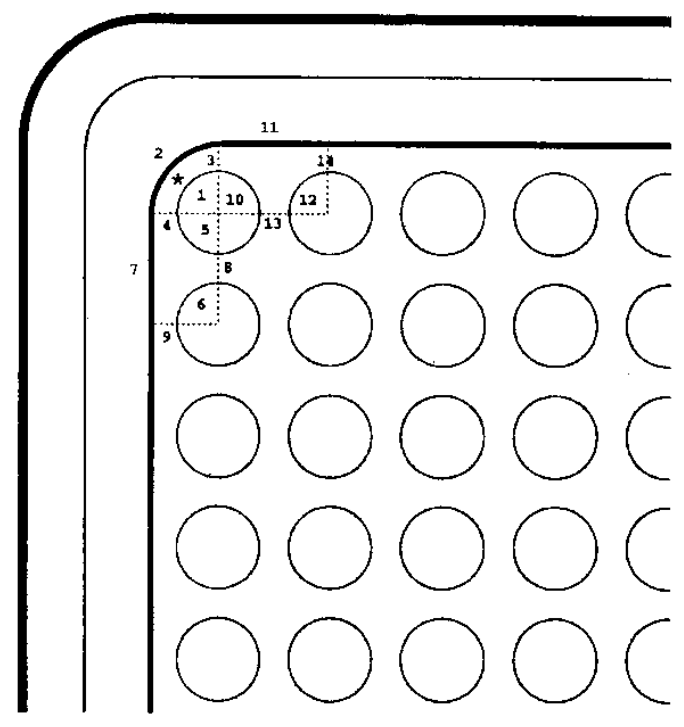

Figure 10.6: Radiation Geometry Type 6 


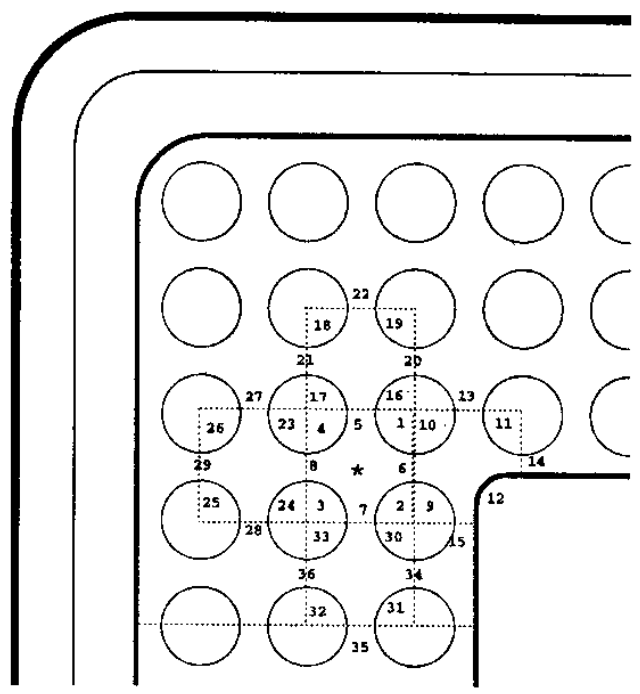

Figure 10.7: Radiation Geometry Type 10

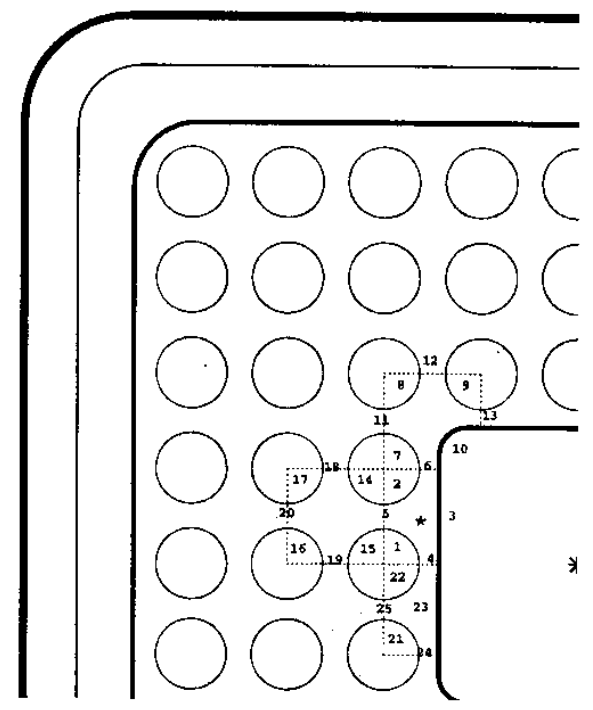

Figure 10.9: Radiation Geometry Type 12

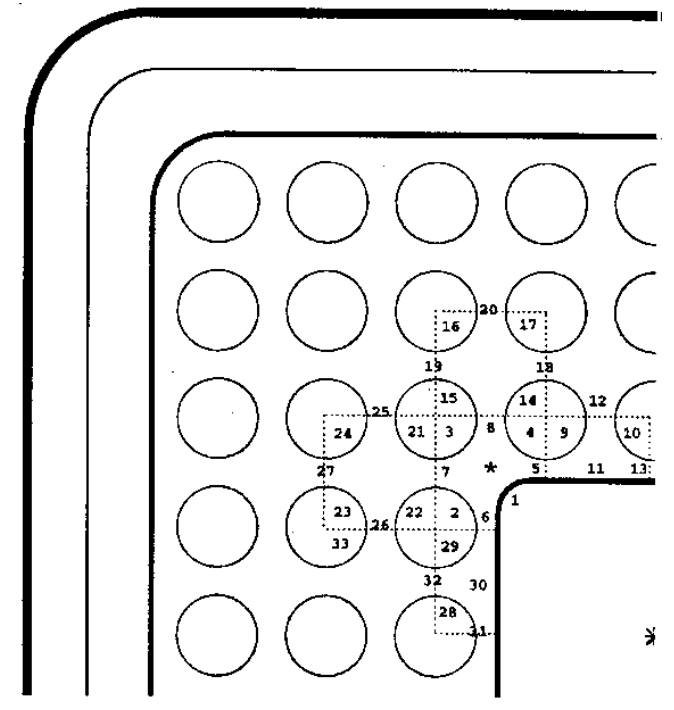

Figure 10.8: Radiation Geometry Type 11

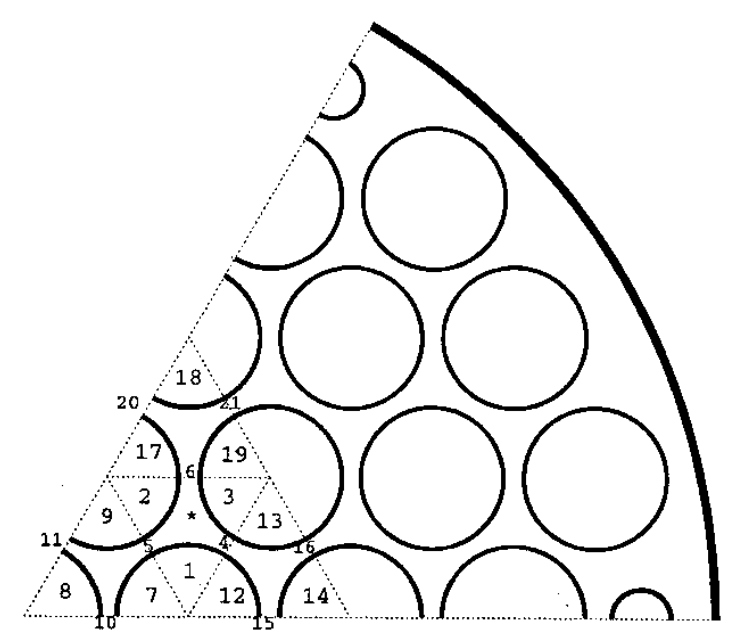

Figure 10.10: Radiation Geometry Type 13 


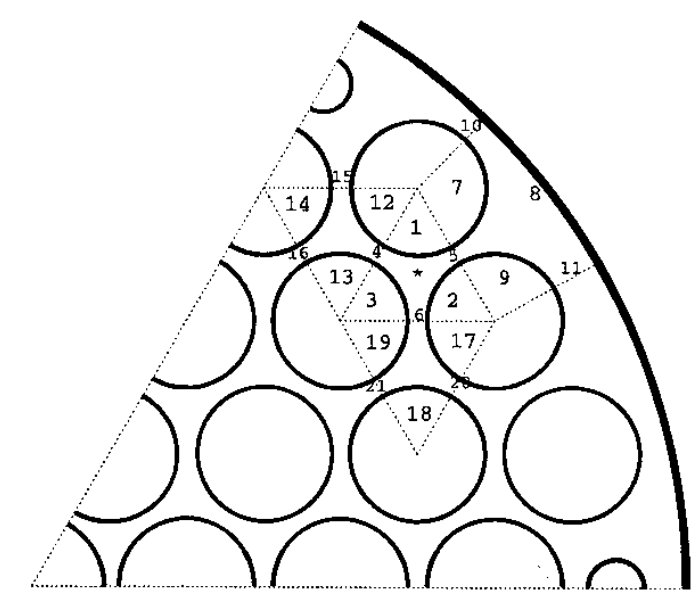

Figure 10.11: Radiation Geometry Type 14

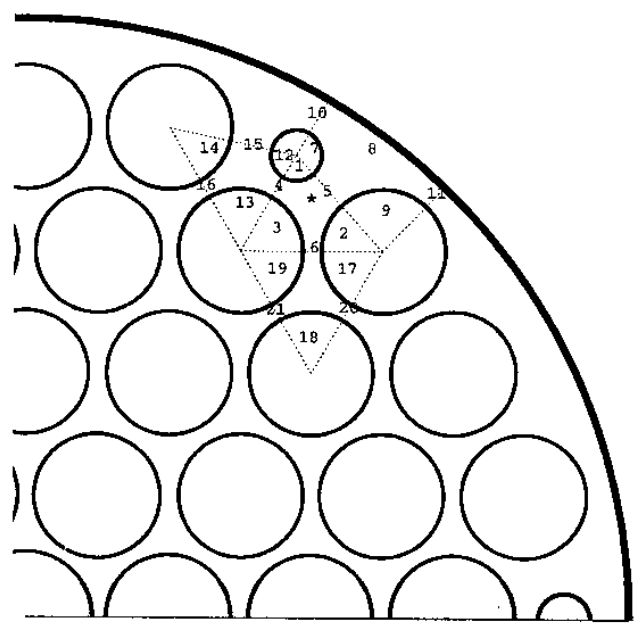

Figure 10.13: Radiation Geometry Type 16

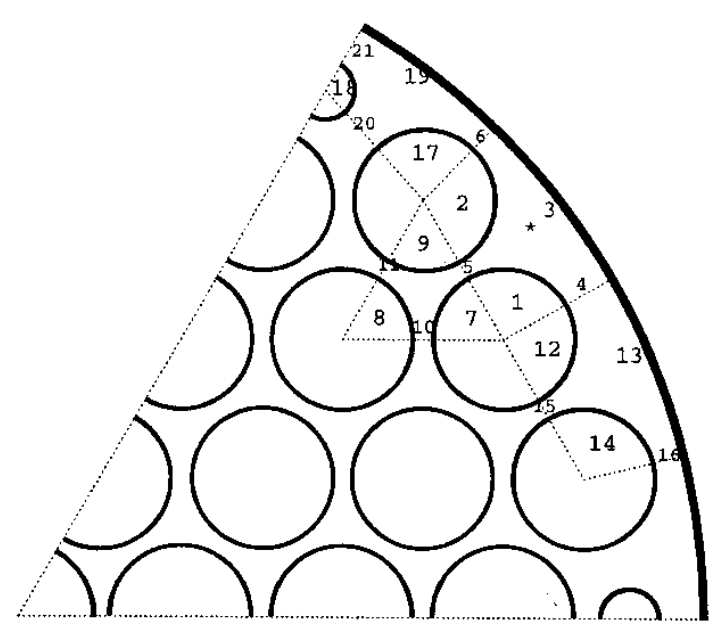

Figure 10.12: Radiation Geometry Type 15

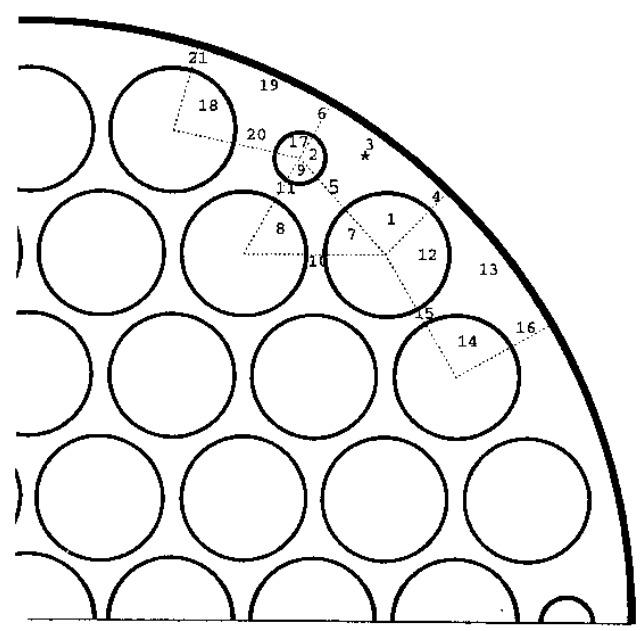

Figure 10.14: Radiation Geometry Type 17 
CARD 8.10.1: LRAD (IRAD, J); J = 1 :NRRAD (IRAD)

\begin{tabular}{|c|c|c|c|}
\hline 8.10 .1 & LRAD (IRAD, J) & {$[-]$} & {$[-]$} \\
\hline \multicolumn{4}{|c|}{$\begin{array}{l}\text { Surface number in position } J \text { for appropriate radiation subchannel IRAD corresponding to location typ } \\
\text { LOCATE(IRAD). Negative for an inside surface. }\end{array}$} \\
\hline Integer & Conditional - if & & \\
\hline
\end{tabular}

\subsection{Cavity to Cavity Radiation Weighting Factor}

CARD 8.10.2: $\operatorname{CAVSM}($ IRAD, J); $\mathrm{J}=1: 4$

Four records per card are required.

\begin{tabular}{|c|c|c|c|}
\hline 8.10 .2 & CAVSM (IRAD, J) & {$[-]$} & {$[-]$} \\
\hline \multicolumn{4}{|c|}{ Weighting factor to be applied to cavity-to-cavity radiation: } \\
\hline \multicolumn{4}{|c|}{1.0 - Suggested value } \\
\hline Integer & Conditional - if & & \\
\hline
\end{tabular}

\subsection{Radiation Location Type Information}

CARD 8.11: IDTYP(I); I = $1:$ NLTYP

\begin{tabular}{|c|c|c|c|}
\hline 8.11 & IDTYP (I) & {$[-]$} & {$[-]$} \\
\hline \multicolumn{4}{|c|}{ Location type to be input: } \\
\hline \multicolumn{4}{|c|}{$>0$ - Manual input to follow } \\
\hline \multicolumn{4}{|c|}{$<0$ - Auto view factor routine to be used } \\
\hline Integer & Condition & & \\
\hline Any & & & \\
\hline
\end{tabular}

Manual Location Type Input

If IDTYP(I) < 0, skip CARD 8.11.1 through CARD 8.11.4

$\underline{\text { Area Input }}$ 
CARD 8.11.1: $\operatorname{ARAD}(\mathrm{J}) ; \mathrm{J}=1: \mathrm{JTOT}$

JTOT is the total number of surfaces for location type IDTYP(I).

\begin{tabular}{|l|l|c|c|}
\hline $\mathbf{8 . 1 1 . 1}$ & $\operatorname{ARAD}(\mathrm{J})$ & {$\left[\mathrm{cm}^{2}\right]$} & {$\left[\mathrm{in}^{2}\right]$} \\
\hline Surface area of position J for location type IDTYPE(I) & \\
\hline Float & Conditional - if IDTYP $>0$ \\
\hline$>0.0$ & \\
\hline
\end{tabular}

$\underline{\text { Emissivity Input }}$

CARD 8.11.2: $\operatorname{ERAD}(\mathrm{J}) ; \mathrm{J}=1: \mathrm{JTOT}$

JTOT is the total number of surfaces for location type IDTYP(I).

\begin{tabular}{|l|l|l|l|}
\hline $\mathbf{8 . 1 1 . 2}$ & $\operatorname{ERAD}(\mathrm{J})$ & {$[-]$} & {$[-]$} \\
\hline Emissivity of position J for location type IDTYPE(I) & \\
\hline Float & Conditional - if IDTYP $>0$ \\
\hline$>0.0$ & \\
\hline
\end{tabular}

$\underline{\text { View Factor Input }}$

CARD 8.11.3: $\operatorname{FRAD}(\mathrm{J}, \mathrm{K}) ; \mathrm{J}=1: \mathrm{JL}, \mathrm{K}=\mathrm{J}: \mathrm{JL}$

JL is the total number of radiant surfaces in location type IDTYP(I).

\begin{tabular}{|c|c|c|c|}
\hline 8.11 .3 & $\operatorname{FRAD}(\mathrm{J}, \mathrm{K})$ & {$[-]$} & {$[-]$} \\
\hline \multicolumn{4}{|c|}{$\begin{array}{l}\text { Radiation view factor between surface } \mathrm{J} \text { and surface } \mathrm{K} \text { where } \mathrm{J}<\mathrm{K} \\
\text { Continue until all } \mathrm{J} \text { surfaces have been entered, starting each } \mathrm{J} \text { surface group with a new card set. } \\
\text { Eight records are required per card set. }\end{array}$} \\
\hline Float & Conditional & & \\
\hline
\end{tabular}

$\underline{\text { Beam Length Input }}$

CARD 8.11.4: $\operatorname{DRAD}(\mathrm{J}, \mathrm{K}) ; \mathrm{J}=1: \mathrm{JL}, \mathrm{K}=\mathrm{J}: \mathrm{JL}$

JL is the total number of radiant surfaces in location type IDTYP(I).

\begin{tabular}{|c|c|c|c|}
\hline 8.11 .4 & $\operatorname{DRAD}(J, K)$ & {$[-]$} & {$[-]$} \\
\hline \multicolumn{4}{|c|}{$\begin{array}{l}\text { Beam length between surface } \mathrm{J} \text { and surface } \mathrm{K} \text { where } \mathrm{J}<\mathrm{K} \\
\text { Continue until all } \mathrm{J} \text { surfaces have been entered, starting each } \mathrm{J} \text { surface group with a new card set. } \\
\text { Eight records are required per card set. }\end{array}$} \\
\hline Float & Conditional & & \\
\hline \multicolumn{4}{|l|}{$>0.0$} \\
\hline
\end{tabular}


Repeat CARD 8.11 through CARD 8.11.4 until all IDTYP(I) values are entered for IDTYP(I) > 0 .

Auto View Factor Input

Omit if $\operatorname{IDTYP}(\mathrm{I})>0$.

CARD 8.11.5: APAR(III); III $=1: 10$

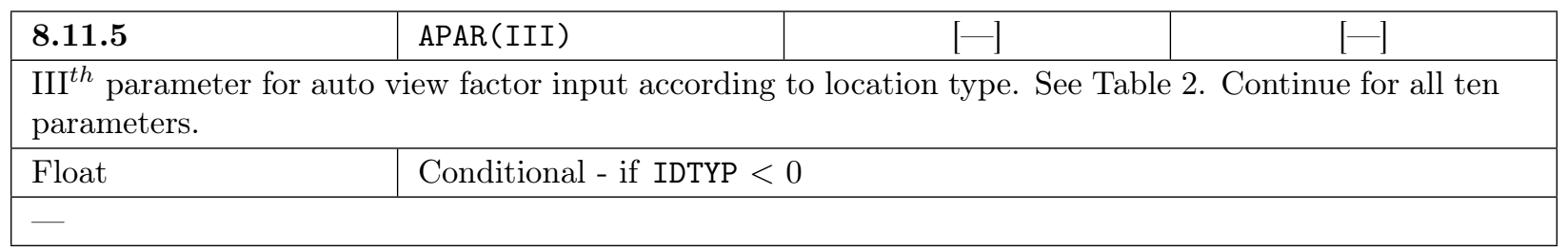

Table 2: Summary of View Factor Inputs

\begin{tabular}{|l|c|c|c|c|c|c|c|c|c|c|}
\hline Radiation Channel Type & Apar1 & Apar2 & Apar3 & Apar4 & Apar5 & Apar6 & Apar7 & Apar8 & Apar9 & Apar10 \\
\hline Type 1 & RD & RE & LRD & RP & - & - & - & - & - & - \\
\hline Type 2 & RD & RE & RWG & RP & 0 & 0 & WE & LRD & - & - \\
\hline Type 3 & RD & RE & RWG & RP & 0 & 0 & WE & LRD & - & - \\
\hline Type 4 & RD & RE & RWG & RP & 0 & 0 & WE & - & - & - \\
\hline Type 5 & RD & RE & RWG & RP & 0 & 0 & WE & - & - & - \\
\hline Type 6 & RD & RE & RWG & RP & 0 & 0 & WE & - & - & - \\
\hline Type 10 & RD & RE & WCR & RP & HWW & DWB & WCE & - & - & - \\
\hline Type 11 & RD & RE & WCR & RP & HWW & DWB & WCE & - & - & - \\
\hline Type 12 & RD & RE & WCR & RP & HWW & DWB & WCE & - & - & - \\
\hline Type 13 & R1D & R1E & R2D & R2E & R3D & R3E & P1:2 & P1:3 & P2:3 & - \\
\hline Type 14 & R1D & R1E & R2D & R2E & R3D & R3E & P1:3 & P1:3 & P2:3 & - \\
\hline Type 15 & R1D & R1E & R2D & R2E & R3D & R3E & D1T & D2T & G1W & G2W \\
\hline
\end{tabular}

Key for Table 2:

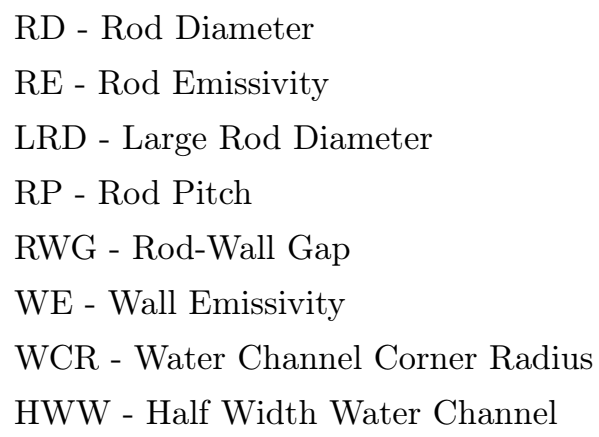


DWB - Distance from Water Channel Centerline to Radius of the Channel Boundary

WCE - Water Channel Emissivity

R1D - Rod 1 Diameter

R2D - Rod 2 Diameter

R3D - Rod 3 Diameter

R1E - Rod 1 Emissivity

R2E - Rod 2 Emissivity

R3E - Rod 3 Emissivity

P1:2 - Pitch 1-2

P1:3 - Pitch 1-3

P2:3 - Pitch 2-3

D1T - Distance from Tube Centerline to Rod 1 Centerline

D2T - Distance from Tube Centerline to Rod 2 Centerline

G1W - Gap Length from Rod 1 to Tube Wall

G2W - Gap Length from Rod 2 to Tube Wall

Card 8.12 is read only if NCAN $>0$.

CARD 8.12: QNINIT, TLIDEN, EWET

\begin{tabular}{|l|l|c|c|}
\hline $\mathbf{8 . 1 2}$ & QNINIT & {$[\mathrm{s}]$} & {$[\mathrm{s}]$} \\
\hline Time at which canister quench is initiated & & \\
\hline Float & Conditional - if NCAN $>0$ & \\
\hline$>=0.0$ & \\
\hline
\end{tabular}

\begin{tabular}{|c|c|c|c|}
\hline 8.12 & TLIDEN & {$\left[{ }^{\circ} \mathrm{C}\right]$} & {$\left[{ }^{\circ} \mathrm{F}\right]$} \\
\hline \multicolumn{4}{|c|}{ Temperature at Leidenfrost point, at which: $\frac{d q^{\prime \prime}}{d\left(T_{\text {wall }}-T_{\text {sat }}\right)}=0$} \\
\hline Float & Conditio & & \\
\hline$>0.0$ & & & \\
\hline
\end{tabular}

\begin{tabular}{|l|l|c|c|}
\hline $\mathbf{8 . 1 2}$ & EWET & {$[-]$} & {$[-]$} \\
\hline Emissivity of the canister & & \\
\hline Float & Conditional - if NCAN $>0$ \\
\hline$>0.0$ & \\
\hline
\end{tabular}

Card 8.13 is read only for parallel runs - if run_parallel == True

CARD 8.13: N, ROD_GLOBAL (N), OWNER, DOMAINS_W_ROD_GHOSTS $(\mathrm{N}, \mathrm{L}) ;$ L $=1: 3$ 


\begin{tabular}{|l|l|c|c|}
\hline $\mathbf{8 . 1 3}$ & $\mathrm{N}$ & {$[-]$} & {$[-]$} \\
\hline Rod ID & Conditional - if run_parallel ==1 \\
\hline Integer & \\
\hline \multicolumn{2}{|l|}{ Local rod index in this parallel domain } \\
\hline
\end{tabular}

\begin{tabular}{|l|l|c|c|}
\hline $\mathbf{8 . 1 3}$ & ROD_GLOBAL $(\mathrm{N})$ & {$[-]$} & {$[-]$} \\
\hline The global index of the rod in the model. & \\
\hline Integer & Conditional - if run_parallel $==1$ \\
\hline$>0$ and $<=$ number of rods in model \\
\hline
\end{tabular}

\begin{tabular}{|l|l|l|l|}
\hline $\mathbf{8 . 1 3}$ & OWNER & {$[-]$} & {$[-]$} \\
\hline \multicolumn{2}{|l|}{ The solution domain that owns this rod. } \\
\hline Integer & Conditional - if run_parallel == \\
\hline Parallel domain index in model \\
\hline
\end{tabular}

Card 8.14 is read only if IKFUEL $\geq 0$. Cards 8.14.1 and 8.14.2 are read in pairs for each rod N.

CARD 8.14.1: N

\begin{tabular}{|l|l|l|l|}
\hline $\mathbf{8 . 1 4 . 1}$ & $\mathrm{N}$ & {$[-]$} & {$[-]$} \\
\hline Index of rod & Conditional - if IKFUEL $\geq 0$ \\
\hline Integer & \\
\hline Must be rod index defined in model \\
\hline
\end{tabular}

CARD 8.14.2: $\operatorname{EXPOSURE}(\mathrm{N}, \mathrm{J}), \operatorname{GAdCON}(\mathrm{N}, \mathrm{J}), \operatorname{ImOX}(\mathrm{N}, \mathrm{J}) ; \mathrm{J}=1: \operatorname{NONODE}$

\begin{tabular}{|l|l|l|l|}
\hline $\mathbf{8 . 1 4 . 2}$ & $\operatorname{EXPOSURE}(\mathrm{N}, \mathrm{J})$ & {$[\mathrm{GWd} / \mathrm{t}]$} & {$[\mathrm{GWd} / \mathrm{t}]$} \\
\hline Burnup of fuel rod $\mathrm{N}$ at axial node $\mathrm{J}$ & \\
\hline Float & Conditional - if IKFUEL $\geq 0$ \\
\hline$>=0.0$ & \\
\hline
\end{tabular}

\begin{tabular}{|l|l|l|l|}
\hline $\mathbf{8 . 1 4 . 2}$ & GADCON $(\mathrm{N}, \mathrm{J})$ & {$[-]$} & {$[-]$} \\
\hline Fractional amount of $\mathrm{Gd}$ per $\mathrm{UO}_{2}$ or $\mathrm{Pu}$ content of MOX fuel rod $\mathrm{N}$ at axial node $\mathrm{J}$ \\
\hline Float & Conditional - if IKFUEL $\geq 0$ \\
\hline$>=0.0$ & \\
\hline
\end{tabular}




\begin{tabular}{|l|l|l|}
\hline 8.14.2 & IMOX $(\mathrm{N})$ & {$[-]$} \\
\hline The burnup-dependent fuel thermal conductivity option for rod $\mathrm{N}$ at axial node $\mathrm{J}:$ \\
1. - Modified NFI correlation ( $\mathrm{UO}_{2}$ fuel) \\
2. - Halden correlation $\left(\mathrm{UO}_{2}\right.$ fuel) \\
3. - Duriez/Modified NFI correlation (MOX fuel) \\
4. - Halden correlation (MOX fuel) \\
5. - Amaya correlation (MOX fuel) \\
\hline Integer \\
\hline-
\end{tabular}

Card 8.15 is only read if a negative value is entered for "NUM_SUBAX" in Card 8.2. Note that all "NUM_SUBAX" values must be the same if using this option. This input is only used when using the ROTHCON feature for reconstructing rod surface heat transfer behavior.

\begin{tabular}{|c|c|c|c|}
\hline 8.15 & TABLEID & {$[-]$} & {$[-]$} \\
\hline \multicolumn{4}{|c|}{$\begin{array}{l}\text { The ID of this table being entered. This value must match the absolute value of the value entered for } \\
\text { "NUM_SUBAX". }\end{array}$} \\
\hline Integer & \multicolumn{3}{|c|}{ Conditional - if NUM_SUBAX $<0$} \\
\hline$>0$ & & & \\
\hline
\end{tabular}

\begin{tabular}{|l|l|c|}
\hline $\mathbf{8 . 1 5}$ & NLEV & {$[-]$} \\
\hline $\begin{array}{l}\text { The number of values in the table. Note that this must match the number of levels that will be in the } \\
\text { rod. See the Theory Manual for information on how rods are meshed. }\end{array}$ \\
\hline Integer & Conditional - if NUM_SUBAX $<0$ \\
\hline$>0$ &
\end{tabular}

\begin{tabular}{|l|l|l|l|}
\hline $\mathbf{8 . 1 5}$ & NUM_SUBAX (NLEV) & {$[-]$} & {$[-]$} \\
\hline Enter NLEV values, with each value giving the number of subdivisions of each rod level. \\
\hline Integer & Conditional - if NUM_SUBAX $<0$ \\
\hline$>=1$ &
\end{tabular}




\section{Card Group 9}

This card group is read by subroutine READ_CARD_9.

The geometry types are read in this group. The geometry types are numbered sequentially in the order in which they are read. Nuclear rod geometry types are read using cards Card 9.2 through Card 9.5. All other geometry types are read using cards Card 9.6 and Card 9.7.

The first line indicates the group number: NGROUP $=9$

CARD 9.1: NFUELT, IRELF, ICONF, IMWR, IFSWELL, IFDENS, IFRELOC, IFCLADCREEP, NDUM10, NDUM11, NDUM12, NDUM13, NDUM14

\begin{tabular}{|l|l|c|c|}
\hline $\mathbf{9 . 1}$ & NFUELT & {$[-]$} & {$[-]$} \\
\hline $\begin{array}{l}\text { Number of geometry types to be read } \\
\text { Note: A geometry type may be used by both rods and unheated conductors, but for the unheated conductor } \\
\text { any heat generation specificed for that type will be ignored. }\end{array}$ \\
\hline Integer & Required \\
\hline$>=0$ &
\end{tabular}

\begin{tabular}{|c|c|c|c|}
\hline 9.1 & IRELF & {$[-]$} & {$[-]$} \\
\hline \multicolumn{4}{|c|}{ Flag for fuel relocation-induced thermal conductivity degradation: } \\
\hline \multicolumn{4}{|l|}{$1-$ On } \\
\hline \multicolumn{4}{|l|}{$0-$ Off } \\
\hline \multicolumn{4}{|c|}{ This flag is used for the thermal conductivity degradation due to the fuel relocation. } \\
\hline Integer & Require & & \\
\hline
\end{tabular}




\begin{tabular}{|l|l|l|l|}
\hline $\mathbf{9 . 1}$ & ICONF & {$[-]$} & {$[-]$} \\
\hline Fuel degradation flag: & & \\
1 - On & \\
0 - Off & \\
Note: If IRELF $=1$, then ICONF $=1$ & \\
\hline Integer & Required & \\
\hline- & \\
\hline
\end{tabular}

\begin{tabular}{|c|c|c|c|}
\hline 9.1 & IMWR & {$[-]$} & {$[-]$} \\
\hline \multicolumn{4}{|c|}{ Flag for metal-water reaction (zirconium dioxide only): } \\
\hline \multicolumn{4}{|l|}{$0-$ Off } \\
\hline \multicolumn{4}{|c|}{1 - Cathcart (for best-estimate analysis) } \\
\hline \multicolumn{4}{|c|}{2 - Baker-Just (for evaluation model analysis) } \\
\hline Integer & Required & & \\
\hline- & & & \\
\hline
\end{tabular}

\begin{tabular}{|c|c|c|c|}
\hline 9.1 & IFSWELL & {$[-]$} & {$[-]$} \\
\hline \multicolumn{4}{|c|}{ Flag for fuel swelling: } \\
\hline \multicolumn{4}{|l|}{$0-$ Off } \\
\hline \multicolumn{4}{|c|}{1 - On, Matpro model for solid and Mod_frapcon model for gaseous fission product swelling } \\
\hline \multicolumn{4}{|c|}{2 - On, Matpro model for both solid and gaseous fission product swelling } \\
\hline \multicolumn{4}{|c|}{$\begin{array}{l}\text { This flag is used to choose the models to account for fuel swelling induced by both solid and gaseous } \\
\text { fission products, and the total amount of fuel swelling is used to calculate the corresponding gap } \\
\text { conductance. }\end{array}$} \\
\hline Integer & Required & & \\
\hline- & & & \\
\hline
\end{tabular}




\begin{tabular}{|l|l|l|}
\hline $\mathbf{9 . 1}$ & IFDENS & {$[-]$} \\
\hline Fuel densification flag: \\
0 - Off \\
1 - On, Matpro model is used \\
2 - On, Frapcon model is used \\
3 - On, Escore model is used \\
This flag is used to choose the models to account for fuel densification, which is used to calculate the \\
corresponding gap conductance. \\
\hline Integer & Required \\
\hline
\end{tabular}

\begin{tabular}{|l|l|l|}
\hline $\mathbf{9 . 1}$ & IFRELOC & {$[-]$} \\
\hline Flag for Fuel relocation induced gap conductance change: \\
0 - Off \\
1 - On, Frapcon model is used \\
2 - On, Escore model is used \\
3 - On, Modified Escore model is used \\
This flag is used to choose the model to account for fuel relocation, which is used to calculate the \\
corresponding gap conductance. \\
\hline Integer & Required \\
\hline -
\end{tabular}

\begin{tabular}{|l|l|c|}
\hline $\mathbf{9 . 1}$ & IFCLADCREEP & {$[-]$} \\
\hline Clad creep model: \\
0 - Off \\
1 - On, the Bison model is used \\
2 - On, the Escore model is used \\
This flag is used to choose the model to account for clad creep, which is used to calculate the corre- \\
sponding gap conductance. \\
\hline Integer & Required \\
\hline- &
\end{tabular}




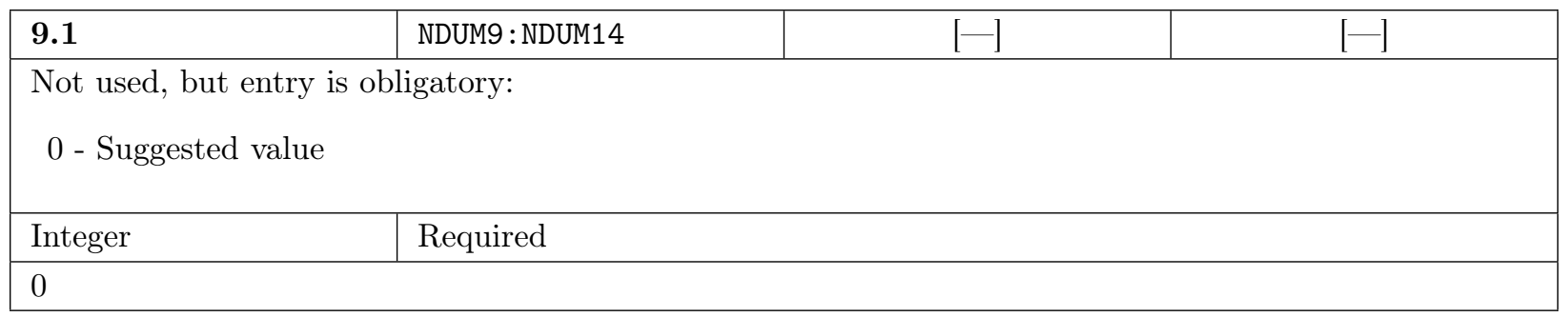

\section{$11.1 \quad$ Nuclear Fuel Geometry Types}

Cards CARD 9.2 through CARD 9.5 are read only for nuclear fuel geometry types. If FTYPE(I) $\neq$ NUCL,

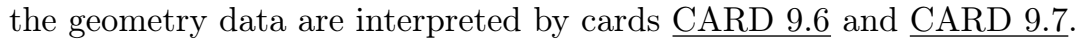

CARD 9.2: I, FTYPE(I), DROD, DFUEL(I), NFUEL, IMATF, IMATC, IMATOX(I), DCORE, TCLAD, FTDENS(I), IGPC, IGFORC, IRADP, EPSO

\begin{tabular}{l}
\begin{tabular}{|l|l|c|c|}
\hline $\mathbf{9 . 2}$ & $\mathrm{I}$ & {$[-]$} & {$[-]$} \\
\hline $\begin{array}{l}\text { Geometry type identification number } \\
\text { Note: Geometry type index numbers must be entered sequentially from 1 to NFUEL T. Skipping numbers } \\
\text { is not permitted. }\end{array}$ \\
\hline Integer & Conditional - if FTYPE(I) = nucl \\
\hline-
\end{tabular} \\
\hline
\end{tabular}

\begin{tabular}{|l|l|l|l|}
\hline $\mathbf{9 . 2}$ & FTYPE(I) & {$[-]$} & {$[-]$} \\
\hline $\begin{array}{l}\text { Alphanumeric geometry type flag: } \\
\text { nucl - nuclear fuel rod }\end{array}$ \\
\hline Character & Conditional - if FTYPE(I) = nucl \\
\hline -
\end{tabular}

\begin{tabular}{|l|l|c|c|}
\hline $\mathbf{9 . 2}$ & DROD & {$[\mathrm{m}]$} & [in] \\
\hline Outside rod diameter & Conditional - if FTYPE (I) = nucl \\
\hline Float & \\
\hline$>0.0$ & \\
\hline
\end{tabular}

\begin{tabular}{|l|l|c|c|}
\hline $\mathbf{9 . 2}$ & DFUEL (I) & {$[\mathrm{m}]$} & [in] \\
\hline Fuel pellet diameter & Conditional - if FTYPE(I) = nucl \\
\hline Float & \\
\hline$>0.0$ & \\
\hline
\end{tabular}




\begin{tabular}{|l|l|c|c|}
\hline $\mathbf{9 . 2}$ & NFUEL & {$[-]$} & {$[-]$} \\
\hline \multicolumn{2}{|l|}{ Number of radial nodes in fuel pellet } & \\
\hline \multicolumn{2}{|l|}{ Integer } & Conditional - if FTYPE(I) = nucl \\
\hline$>0$ & \\
\hline
\end{tabular}

\begin{tabular}{|c|c|c|c|}
\hline 9.2 & IMATF & {$[-]$} & {$[-]$} \\
\hline \multicolumn{4}{|c|}{ Fuel material properties flag: } \\
\hline \multicolumn{4}{|c|}{0 - built-in $\mathrm{UO}_{2}$ properties } \\
\hline \multicolumn{4}{|c|}{$>0$ - number of the material properties table (see Card 10) } \\
\hline Integer & Condit & & \\
\hline- & & & \\
\hline
\end{tabular}

\begin{tabular}{|c|c|c|c|}
\hline 9.2 & IMATC & {$[-]$} & {$[-]$} \\
\hline \multicolumn{4}{|c|}{ Clad material properties flag: } \\
\hline \multicolumn{4}{|c|}{0 - built-in zirconium dioxide properties } \\
\hline \multicolumn{4}{|c|}{$>0$ - number of the material properties table (see Card 10) } \\
\hline Integer & Condit & & \\
\hline- & & & \\
\hline
\end{tabular}

\begin{tabular}{|c|c|c|c|}
\hline 9.2 & IMATOX & {$[-]$} & {$[-]$} \\
\hline \multicolumn{4}{|c|}{ Clad oxide properties flag: } \\
\hline \multicolumn{4}{|c|}{0 - built-in zirconium dioxide properties } \\
\hline \multicolumn{4}{|c|}{$>0$ - number of the material properties table (see Card 10) } \\
\hline Integer & Conditi & & \\
\hline - & & & \\
\hline
\end{tabular}

\begin{tabular}{|c|c|c|c|}
\hline 9.2 & DCORE & {$[\mathrm{m}]$} & [in] \\
\hline \multicolumn{4}{|c|}{ Diameter of central void (for cored fuel) } \\
\hline \multicolumn{4}{|c|}{0 - if uncored fuel (solid pellet) } \\
\hline Float & Condit & & \\
\hline$>=0.0$ & & & \\
\hline
\end{tabular}




\begin{tabular}{|l|l|c|c|}
\hline $\mathbf{9 . 2}$ & TCLAD & {$[\mathrm{m}]$} & {$[\mathrm{in}]$} \\
\hline Clad thickness & Conditional - if FTYPE(I) $=$ nucl \\
\hline Float & \\
\hline$>0.0$ &
\end{tabular}

\begin{tabular}{|c|c|c|c|}
\hline 9.2 & FTDENS (I) & {$[-]$} & {$[-]$} \\
\hline \multicolumn{4}{|c|}{$\begin{array}{l}\text { Fuel theoretical density as a fraction (like } 0.95 \text { ) } \\
\text { Used only if built-in } \mathrm{UO}_{2} \text { properties have been flagged-i.e. if IMATF }=0 \text {. } \\
\text { Note: Do not enter zero }\end{array}$} \\
\hline Float & Conditiona & & \\
\hline
\end{tabular}

\begin{tabular}{|c|c|c|c|}
\hline 9.2 & IGPC & {$[-]$} & {$[-]$} \\
\hline \multicolumn{4}{|c|}{ Gap conductance option flag: } \\
\hline \multicolumn{4}{|c|}{0 - for constant gap conductance (as specified by $\operatorname{HGAP}(\mathrm{N})$ on CARD 8.2) } \\
\hline \multirow{2}{*}{\multicolumn{4}{|c|}{$\begin{array}{l}\text { Enter a positive integer for user-specified non-uniform gap conductance (entered on CARD } 9.4 \text { in a } \\
\text { table of IGPC elements) } \\
\text { Enter a negative integer for the dynamic gap conductance model. There will be } \mid \text { IGPC } \mid \text { entries in the } \\
\text { cold gap width vs. axial location table read on CARD } 9.4\end{array}$}} \\
\hline & & & \\
\hline Integer & Condi & & \\
\hline \multicolumn{4}{|l|}{ 一 } \\
\hline
\end{tabular}

\begin{tabular}{|c|c|c|c|}
\hline 9.2 & IGFORC & {$[-]$} & {$[-]$} \\
\hline \multicolumn{4}{|c|}{$\begin{array}{l}\text { Flag for temporal forcing function on the gap conductance: } \\
\text { Note: valid only if } I G P C>0\end{array}$} \\
\hline \multicolumn{4}{|c|}{0 - constant gap conductance } \\
\hline \multicolumn{4}{|c|}{ Enter a positive integer for temporal forcing function with IGFORC table entries } \\
\hline Integer & Conditi & & \\
\hline - & & & \\
\hline
\end{tabular}

\begin{tabular}{|l|l|l|}
\hline $\mathbf{9 . 2}$ & IRADP & {$[-]$} \\
\hline $\begin{array}{l}\text { Number of entries in radial power profile table for the fuel pellet: } \\
0 \text { - uniform pellet radial power profile }\end{array}$ \\
\hline Integer & Conditional - if FTYPE (I) $=$ nucl \\
\hline$>=0$ &
\end{tabular}




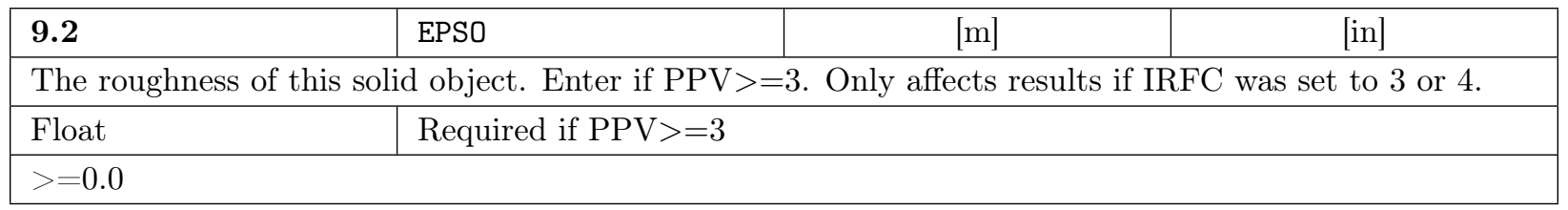

\section{CARD 9.2.5}

This card is a keyword-based optional input and is only valid if FTYPE was "nucl". The keyword will be the parameter name surrounded by curly braces. For example, "clad_rings" is an optional entry. This would be specified as: " $\{$ clad_rings $\}$ ".

\begin{tabular}{|l|l|c|c|}
\hline 9.2.5 & clad_rings & {$[-]$} & {$[-]$} \\
\hline $\begin{array}{l}\text { Number of rings to use in the clad. Uses default value if not specified. The number of rings applies to } \\
\text { the last fuel rod geometry defined on Card 9.2. Not a valid input for any other geometry type. }\end{array}$ \\
\hline Int & Optional - if FTYPE(I) $=$ nucl \\
\hline$>=2$ & \\
\hline
\end{tabular}

Card 9.3 is read only if FTYPE(I)=nucl and IGPC $<0$

CARD 9.3: PGAS(I), VPLEN(I), ROUFF(I), ROUFC(I), (GSFRAC(L); L=1,6), OXIDET

\begin{tabular}{|l|l|c|c|}
\hline $\mathbf{9 . 3}$ & PGAS (I) & [bar $]$ & [psia] \\
\hline Cold pin gas pressure for nuclear fuel rod geometry type I \\
\hline Float & Conditional - if FTYPE(I) $=$ nucl \&\& IGPC $<0$ \\
\hline$>0.0$ & \\
\hline
\end{tabular}

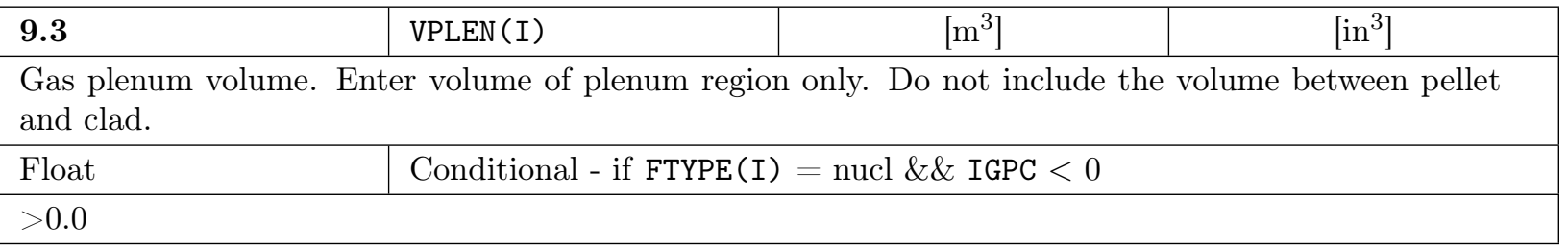

\begin{tabular}{|l|l|c|c|}
\hline $\mathbf{9 . 3}$ & ROUFF (I) & {$[\mathrm{m}]$} & [in] \\
\hline \multicolumn{2}{|l|}{ Fuel pellet surface roughness } & \\
\hline Float & Conditional - if FTYPE (I) $=$ nucl \&\& IGPC $<0$ \\
\hline$>=0.0$
\end{tabular}




\begin{tabular}{|c|c|c|c|}
\hline 9.3 & ROUFC (I) & {$[\mathrm{m}]$} & [in] \\
\hline \multicolumn{4}{|c|}{$\begin{array}{l}\text { Surface roughness of clad inner surface } \\
\text { Note: Fuel and clad surface roughness should correspond to those suggested in Thurgood (1983), as the } \\
\text { correlation is empirical: } \\
\text { fuel surface } \mathrm{ROUFF}=0.000085 \text { in } \\
\text { clad surface } \mathrm{ROUFC}=0.000045 \text { in }\end{array}$} \\
\hline Float & Condition & \& IGF & \\
\hline$>=0.0$ & & & \\
\hline
\end{tabular}

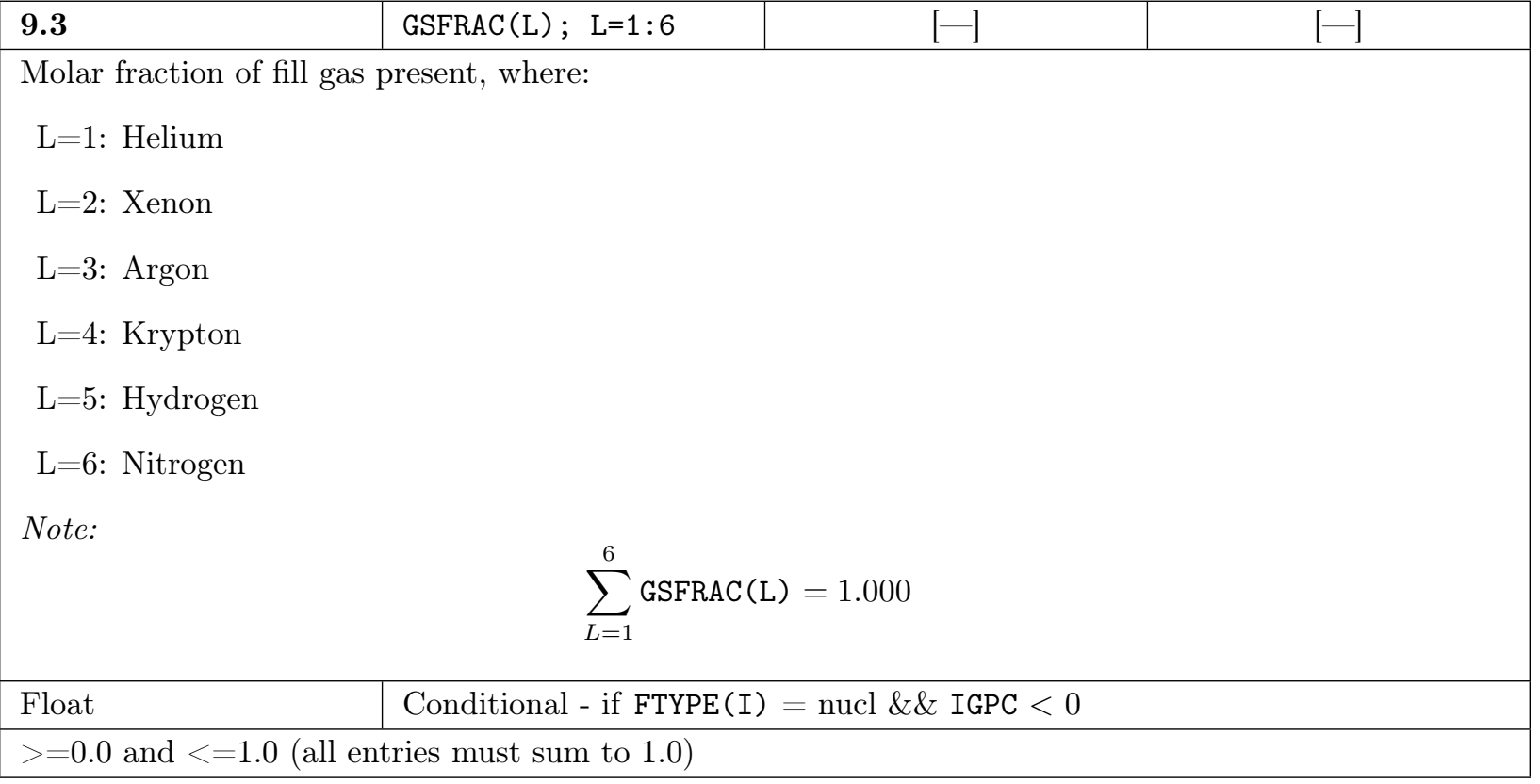

\begin{tabular}{|c|c|c|c|}
\hline 9.3 & OXIDET & [m] & [in] \\
\hline \multicolumn{4}{|c|}{ Initial oxide thickness for the Zircaloy metal-water reaction rate equation (used only if IMWR $>0$ ) } \\
\hline Float & Conditi & $z \mathrm{IG}$ & \\
\hline \multicolumn{4}{|c|}{$>=0.0$} \\
\hline
\end{tabular}

Card 9.4 is read only if FTYPE(I) $=$ nucl and $\mid$ IGPC $\mid>0$

CARD 9.4: $\operatorname{AXJ}(I, L), \operatorname{AGFACT}(I, L) ; L=1:|\operatorname{IGPC}|$ 


\begin{tabular}{|c|c|c|c|}
\hline 9.4 & $\operatorname{AXJ}(I, L)$ & {$[\mathrm{m}]$} & [in] \\
\hline \multicolumn{4}{|c|}{$\begin{array}{l}\text { Top-most vertical position, measured from the bottom of the rod, at which the cold gap width or the } \\
\text { gap conductance AGFACT }(I, L) \text { is applied. (All vertical levels below } \operatorname{AXJ}(I, L) \text { also get that value for } \\
\text { gap width or gap conductance). }\end{array}$} \\
\hline Float & Condition & $\& \mid \mathrm{IC}$ & \\
\hline
\end{tabular}

\begin{tabular}{|c|c|c|c|}
\hline 9.4 & $\operatorname{AGFACT}(I, L)$ & {$\left[\mathrm{m}\right.$ or $\left.\mathrm{W} /\left(\mathrm{m}^{2}-\mathrm{K}\right)\right]$} & {$\left[\right.$ in or btu/(hr-ft $\left.{ }^{2}-^{\circ} \mathrm{F}\right]$} \\
\hline \multicolumn{4}{|c|}{ if IGPC $<0$ : Cold gap width } \\
\hline Float & Conditional - & nucl \&\& $\mid$ IGPC $\mid>0$ & \\
\hline$>0.0$ & & & \\
\hline
\end{tabular}

Card 9.5 is read only if FTYPE $(I)=$ nucl and IRADP $>0$

CARD 9.5: RADP (L), POWR (L); L=1: IRADP

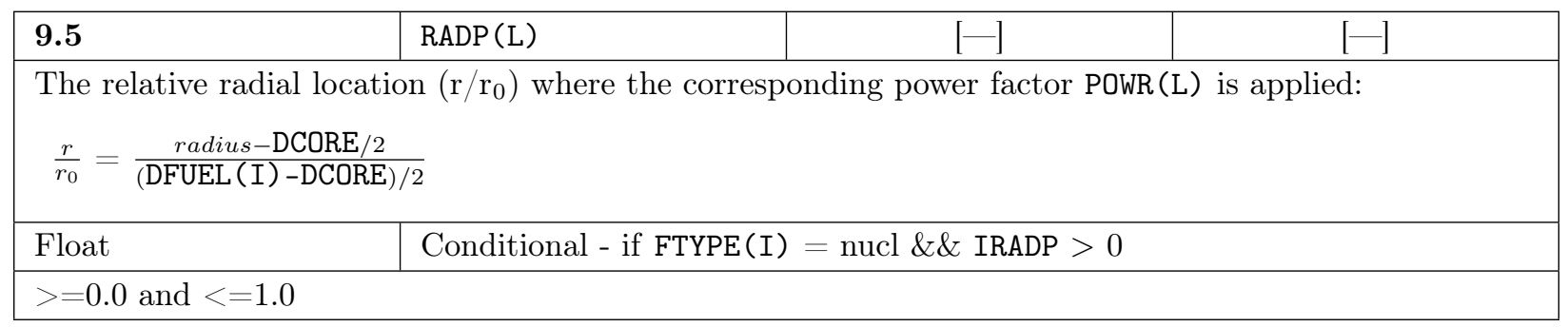

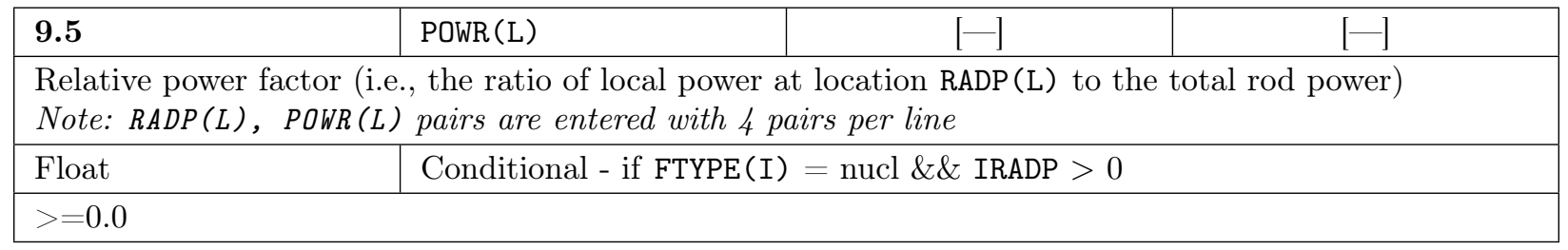

\subsection{Non-Nuclear Geometry Types}

Cards CARD 9.6 and CARD 9.7 are read in pairs for all geometry types that do not describe nuclear fuel (i.e. $\operatorname{FTYPE}(I)=$ HROD, TUBE, WALL).

Card 9.6 is read only if FTYPE (I) $\neq$ NUCL

CARD 9.6: I, FTYPE(I), DROD, DIN, NFUEL, IMATOX(I), IMATIX(I), NDUM8, NDUM9, NDUM10, NDUM11, 
NDUM12, NDUM13, NDUM14, EPSO

\begin{tabular}{|l|l|c|c|}
\hline $\mathbf{9 . 6}$ & I & {$[-]$} & {$[-]$} \\
\hline Geometry type identification number. Must go from 1 to number of geometries and cannot skip indices. \\
\hline Integer & Conditional - if FTYPE(I) $\neq$ nucl \\
\hline- & \\
\hline
\end{tabular}

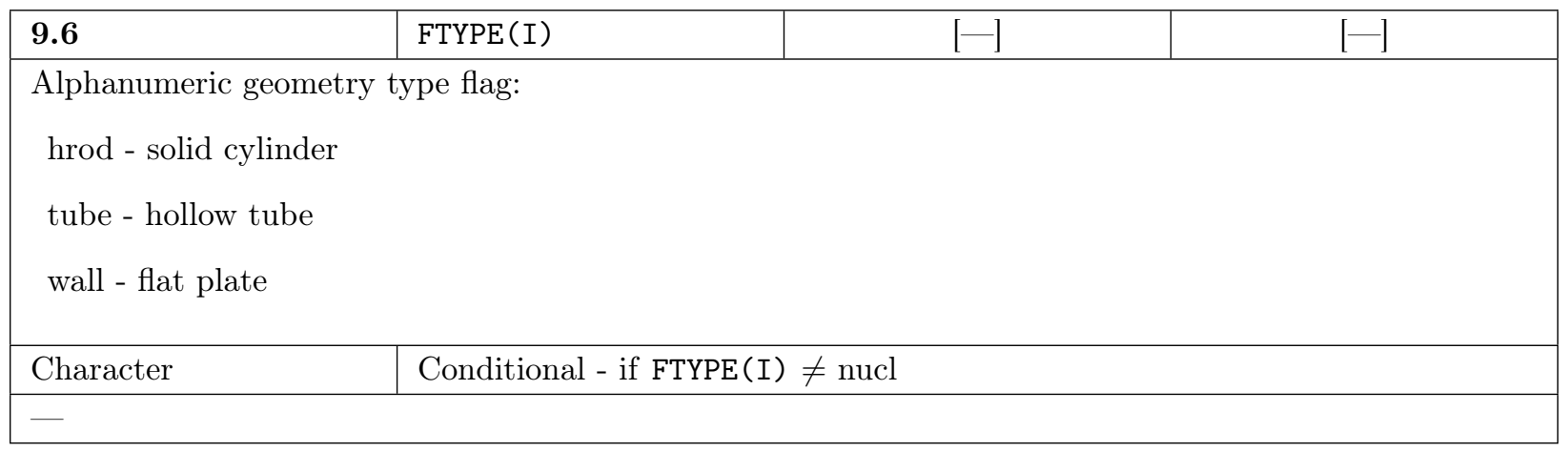

\begin{tabular}{|l|l|l|l|}
\hline $\mathbf{9 . 6}$ & DROD & {$[\mathrm{m}]$} & [in] \\
\hline $\begin{array}{l}\text { Outside diameter for HROD or TUBE geometries, } \underline{\text { or }} \\
\text { Wetted perimeter for WALL geometries }\end{array}$ \\
\hline Float & Conditional - if FTYPE $(\mathrm{I}) \neq$ nucl \\
\hline$>0.0$ & \\
\hline
\end{tabular}

\begin{tabular}{|l|l|l|l|}
\hline $\mathbf{9 . 6}$ & DIN & {$[\mathrm{m}]$} & \\
\hline \multicolumn{2}{|c|}{ Inside diameter for TUBE geometries, or } \\
Thickness for WALL geometries, $\underline{\text { or }}$ \\
Enter "0.0" for HROD geometries \\
\hline Float & Conditional - if FTYPE(I) $\neq$ nucl \\
\hline$>0.0$ & \\
\hline
\end{tabular}

\begin{tabular}{|c|c|c|c|}
\hline 9.6 & NFUEL & {$[-]$} & {$[-]$} \\
\hline \multicolumn{4}{|c|}{$\begin{array}{l}\text { Number of radial regions within the conductor. Each region has a uniform power profile and consists } \\
\text { of one material. }\end{array}$} \\
\hline Integer & Condit & & \\
\hline$>0$ & & & \\
\hline
\end{tabular}




\begin{tabular}{|c|c|c|c|}
\hline 9.6 & IMATOX (I) & {$[-]$} & {$[-]$} \\
\hline \multirow{3}{*}{\multicolumn{4}{|c|}{$\begin{array}{l}\text { Material property table identification number for oxide on the outside surface: } \\
0 \text { - built-in zirconium dioxide properties (default) } \\
>0 \text { - index of the material property table for material in region NFUEL if there is no oxide present (see } \\
\text { Card Group 10) }\end{array}$}} \\
\hline & & & \\
\hline & & & \\
\hline Integer & Conditiona & & \\
\hline - & & & \\
\hline
\end{tabular}

\begin{tabular}{|c|c|c|c|}
\hline 9.6 & $\operatorname{IMATIX}(\mathrm{I})$ & {$[-]$} & {$[-]$} \\
\hline \multicolumn{4}{|c|}{$\begin{array}{l}\text { Material property table identification number for oxide on the inside surface (applies only to TUBE or } \\
\text { WALL geometries): }\end{array}$} \\
\hline \multicolumn{4}{|c|}{0 - built-in zirconium dioxide properties (default) } \\
\hline \multicolumn{4}{|c|}{$\begin{array}{l}>0 \text { - index of the material property table for material in region } 1 \text { if there is no oxide present (see } \\
\text { Card Group 10) }\end{array}$} \\
\hline Integer & Conditiona & & \\
\hline
\end{tabular}

\begin{tabular}{|l|l|l|l|}
\hline $\mathbf{9 . 6}$ & NDUM8: NDUM14 & {$[-]$} & {$[-]$} \\
\hline Not used, but entry is obligatory: & \\
0 - Suggested value \\
Note: these dummy values are required as Card 9.6 utilizes the same read statement as Card 9.2 \\
\hline Integer & Required \\
\hline 0 &
\end{tabular}

\begin{tabular}{|l|l|c|c|}
\hline $\mathbf{9 . 6}$ & EPSO & {$[\mathrm{m}]$} & [in] \\
\hline The roughness of this solid object. Enter if PPV $>=3$. Only affects results if IRFC was set to 3 or 4. \\
\hline Float & Required if PPV $>=3$ \\
\hline$>=0.0$ & \\
\hline
\end{tabular}

Card 9.7 is read only if FTYPE(I) $\neq$ NUCL. Data sets for the NFUEL regions of geometry type I are entered starting at the centerline for HROD types and at the inside surface for TUBE and WALL types. Data sets are entered in sequence moving radially toward the outside surface.

CARD 9.7: $\operatorname{NODER}(\mathrm{L}), \operatorname{MATR}(\mathrm{L}), \operatorname{TREG}(\mathrm{L}), \mathrm{QREG}(\mathrm{L}) ; \mathrm{L}=1$ : NFUEL 


\begin{tabular}{|c|c|c|c|}
\hline 9.7 & NODER (L) & {$[-]$} & {$[-]$} \\
\hline \multicolumn{4}{|c|}{$\begin{array}{l}\text { Number of radial heat transfer nodes in region } \mathrm{L} \\
\text { Note: Because of special data handling inside the code, the following restrictions hold: } 2 \leq \text { NODER }(L) \\
\leq N F U E L \text {, where NFUEL is the number of radial regions specified in Card } 9.6\end{array}$} \\
\hline Integer & Condition & & \\
\hline
\end{tabular}

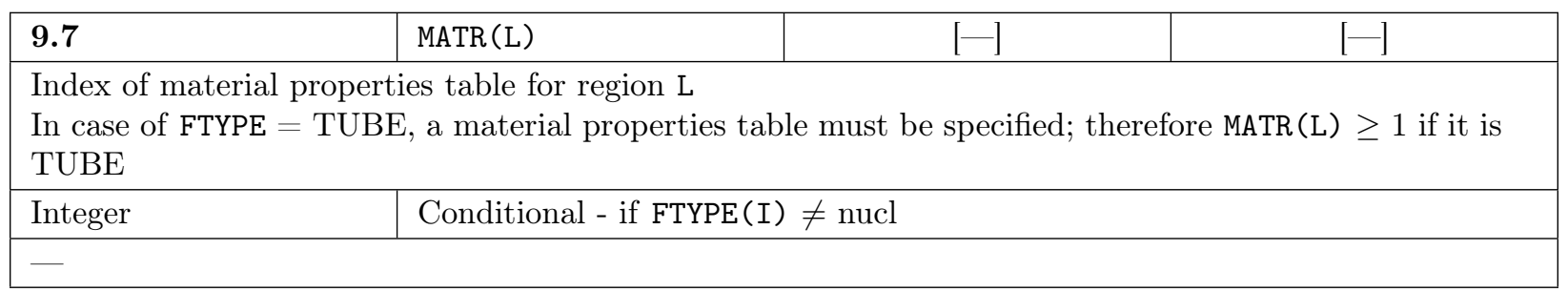

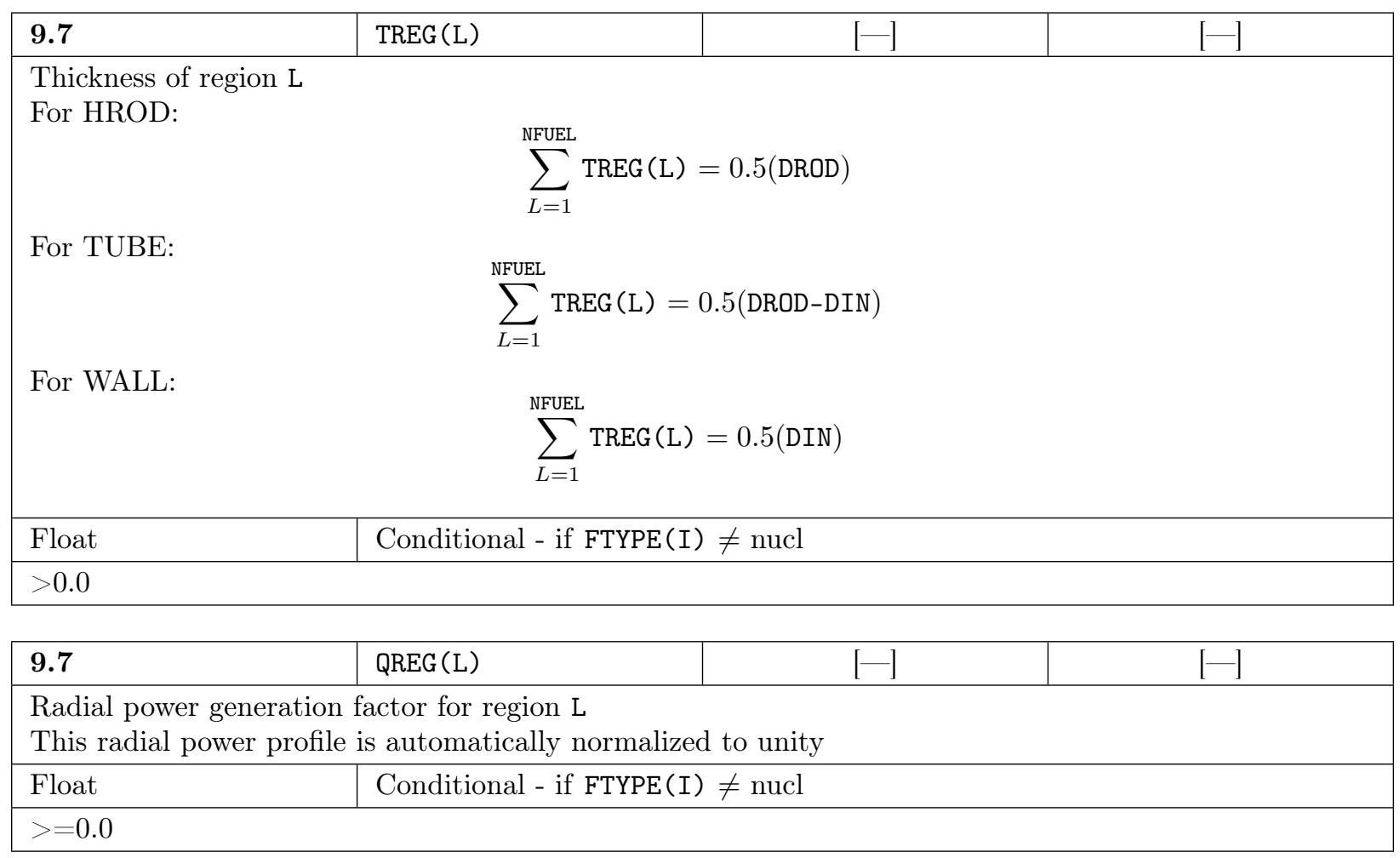




\section{Card Group 10}

This card group is read by subroutine READ_CARD_10.

This input group is required only if user-supplied material properties were flagged by input in CARD GROUP 9 (i.e., with non-zero values for IMATF, IMATC, IMATOX (I), or MATR (L) for any geometry type). If only default material properties are used, (i.e., zircaloy and $\mathrm{UO}_{2}$ ), this group is omitted. In case of FTYPE = TUBE a material properties table has to be specified necessarily, i.e. MATR(L) $\geq 1$.

The first line indicates the group number: NGROUP $=10$

CARD 10.1: NMAT, NDUM2, NDUM3, NDUM4, NDUM5, NDUM6, NDUM7, NDUM8, NDUM9, NDUM10, NDUM11, NDUM12, NDUM13, NDUM14

\begin{tabular}{|l|l|c|c|}
\hline $\mathbf{1 0 . 1}$ & NMAT & {$[-]$} & {$[-]$} \\
\hline \multicolumn{2}{|l|}{ Total number of material properties tables } & \\
\hline Integer & Required & \\
\hline$>=0$ & \\
\hline
\end{tabular}

\begin{tabular}{|c|c|c|c|}
\hline 10.1 & NDUM2 : NDUM14 & {$[-]$} & {$[-]$} \\
\hline \multicolumn{4}{|c|}{ Not used, but entry is obligatory: } \\
\hline Integer & Required & & \\
\hline 0 & & & \\
\hline
\end{tabular}

Cards CARD 10.2 and CARD 10.3 are read in pairs NMAT times.

CARD 10.2: N, NNTDP, RCOLD(N), IMATAN(N)

\begin{tabular}{|l|l|c|c|}
\hline $\mathbf{1 0 . 2}$ & $\mathrm{N}$ & {$[-]$} & {$[-]$} \\
\hline \multicolumn{2}{|l|}{ Material properties table identification number } & \\
\hline Integer & Required \\
\hline$>0$ & \\
\hline
\end{tabular}




\begin{tabular}{|l|l|c|c|}
\hline $\mathbf{1 0 . 2}$ & NNTDP & {$[-]$} & {$[-]$} \\
\hline $\begin{array}{l}\text { Number of entries in materials property table N } \\
\text { Must not exceed 100 }\end{array}$ \\
\hline Integer & Required \\
\hline$>0$ and $<=100$ & \\
\hline
\end{tabular}

\begin{tabular}{|c|c|c|c|}
\hline 10.2 & $\operatorname{RCOLD}(\mathrm{N})$ & {$\left[\mathrm{kg} / \mathrm{m}^{3}\right]$} & {$\left[\mathrm{lbm} / \mathrm{ft}^{3}\right]$} \\
\hline \multicolumn{4}{|c|}{$\begin{array}{l}\text { Cold density for material } \mathrm{N} \\
\text { Note: This value is used to define the mass in the heat transfer nodes composed of material type }\end{array}$} \\
\hline Float & Required & & \\
\hline$>0.0$ & & & \\
\hline
\end{tabular}

\begin{tabular}{|l|l|c|c|}
\hline $\mathbf{1 0 . 2}$ & $\operatorname{IMATAN}(\mathrm{N})$ & {$[-]$} & {$[-]$} \\
\hline Alphanumeric label for material — such as Stainless steel, Inconel 600. Enclose all labels in quotes. \\
\hline Character & Required \\
\hline Any string
\end{tabular}

CARD 10.3: $\operatorname{TPROP}(I, N), \operatorname{CPF} 1(I, N), \operatorname{THCF}(I, N) ; I=1, \operatorname{NNTDP}$

\begin{tabular}{|l|l|l|l|}
\hline $\mathbf{1 0 . 3}$ & $\operatorname{TPROP}(\mathrm{I}, \mathrm{N})$ & {$\left[{ }^{\circ} \mathrm{C}\right]$} & {$\left[{ }^{\circ} \mathrm{F}\right]$} \\
\hline Temperature for entry I in material properties table $\mathrm{N}$ & & \\
\hline Float & Required & \\
\hline$>0.0$ &
\end{tabular}

\begin{tabular}{|l|l|c|c|}
\hline $\mathbf{1 0 . 3}$ & $\operatorname{CPF} 1(\mathrm{I}, \mathrm{N})$ & {$\left[\mathrm{kJ} /\left(\mathrm{kg}-{ }^{\circ} \mathrm{C}\right)\right]$} & {$\left[\mathrm{btu} /\left(\mathrm{lbm}-{ }^{\circ} \mathrm{F}\right)\right]$} \\
\hline Specific heat capacity at temperature TPROP $(\mathrm{I}, \mathrm{N})$ & & \\
\hline Float & Required & \\
\hline$>0.0$
\end{tabular}

\begin{tabular}{|l|l|r|r|}
\hline $\mathbf{1 0 . 3}$ & $\operatorname{THCF}(\mathrm{I}, \mathrm{N})$ & {$[\mathrm{W} /(\mathrm{m}-\mathrm{K})]$} & {$\left[\mathrm{btu} /\left(\mathrm{hr}-\mathrm{ft}-{ }^{\circ} \mathrm{F}\right)\right]$} \\
\hline \multicolumn{2}{|l|}{ Thermal conductivity at temperature TPROP $(\mathrm{I}, \mathrm{N})$} \\
\hline Float & Required & \\
\hline$>0.0$ & \\
\hline
\end{tabular}




\section{Card Group 11}

This card group is read by subroutine READ_CARD_11.

The first line indicates the group number: NGROUP $=11$

CARD 11.1: NQA, NAXP, MNXN, NQ, NGPFF, NQR, NDUM7, NDUM8, NDUM9, NDUM10, NDUM11, NDUM12, NDUM13, NDUM14

\begin{tabular}{|c|c|c|c|}
\hline 11.1 & NQA & {$[-]$} & {$[-]$} \\
\hline \multicolumn{4}{|c|}{ Number of transient changes of the axial power profile: } \\
\hline \multicolumn{4}{|c|}{1 - if the axial power profile is not transient } \\
\hline \multicolumn{4}{|c|}{$\begin{array}{l}\text { Note: When specifying different power factor tables (be they axial, radial, or total power), ensure to } \\
\text { cover the entire transient to be modeled or else there will be error messages in } \hat{A} \breve{A} \ddot{Y} \text { deck.out } \hat{A} \breve{Z} \text { that } \\
\text { 'tabular lookup failed'. For example, if the transient will end at } 1 \text { second, the last set of transient power } \\
\text { factor tables should be for a little over } 1 \text { second. The interpolation functions need to have that 'end cap' } \\
\text { with which to interpolate when determining the current-time power factors. }\end{array}$} \\
\hline Integer & Required & & \\
\hline$>=1$ & & & \\
\hline
\end{tabular}

\begin{tabular}{|l|l|l|l|}
\hline $\mathbf{1 1 . 1}$ & NAXP & {$[-]$} & {$[-]$} \\
\hline Number of axial power profile tables to be read for each transient time point $(\geq 1)$ \\
\hline Integer & Required \\
\hline$>=1$ & \\
\hline
\end{tabular}

\begin{tabular}{|l|l|l|l|}
\hline $\mathbf{1 1 . 1}$ & MNXN & {$[-]$} & {$[-]$} \\
\hline Maximum number of pairs of elements in any axial power profile table & \\
\hline Integer & Required \\
\hline$>=1$
\end{tabular}




\begin{tabular}{|c|c|c|c|}
\hline 11.1 & NQ & {$[-]$} & {$[-]$} \\
\hline \multicolumn{4}{|c|}{$\begin{array}{l}\text { Number of pairs of elements in the total power forcing function table: } \\
0 \text { - if total power is constant }\end{array}$} \\
\hline Integer & Required & & \\
\hline$>=0$ & & & \\
\hline
\end{tabular}

\begin{tabular}{|c|c|c|c|}
\hline 11.1 & NGPFF & {$[-]$} & {$[-]$} \\
\hline \multicolumn{4}{|c|}{ Number of pairs of elements in the gap conductance forcing function table: } \\
\hline \multicolumn{4}{|c|}{0 - if there is no forcing function on gap conductance } \\
\hline Integer & Required & & \\
\hline$>=0$ & & & \\
\hline
\end{tabular}

\begin{tabular}{|c|c|c|c|}
\hline 11.1 & NQR & {$[-]$} & {$[-]$} \\
\hline \multicolumn{4}{|c|}{ Number of radial power profile tables (for different transient points of time): } \\
\hline \multicolumn{4}{|c|}{1 - if radial power profile is not transient } \\
\hline Integer & Required & & \\
\hline \multicolumn{4}{|l|}{$>=1$} \\
\hline 11.1 & NDUM7 : NDUM14 & {$[-]$} & {$[-]$} \\
\hline \multicolumn{4}{|c|}{ Not used, but entry is obligatory: } \\
\hline \multicolumn{4}{|c|}{0 - Suggested value } \\
\hline Integer & Required & & \\
\hline 0 & & & \\
\hline
\end{tabular}

Cards CARD 11.2, CARD 11.3, and CARD 11.4 are read in groups NQA times.

\section{CARD 11.2: YQA}

\begin{tabular}{|l|l|c|c|}
\hline $\mathbf{1 1 . 2}$ & YQA & {$[\mathrm{s}]$} & {$[\mathrm{s}]$} \\
\hline Transient time for each axial power profile change & & \\
\hline Float & Required & \\
\hline$>=0.0$ & \\
\hline
\end{tabular}

Cards CARD 11.3 and CARD 11.4 are read in pairs NAXP times for each transient time YQA (M=1:NQA). CARD 11.3: I, NAXN(I) 


\begin{tabular}{|c|c|c|c|}
\hline 11.3 & I & {$[-]$} & {$[-]$} \\
\hline \multicolumn{4}{|c|}{$\begin{array}{l}\text { Axial power profile table identification number (refer to Card } 8.2 \text { - each rod can have its own axial } \\
\text { power profile table). }\end{array}$} \\
\hline Integer & Required & & \\
\hline- & & & \\
\hline
\end{tabular}

\begin{tabular}{|l|l|l|l|}
\hline $\mathbf{1 1 . 3}$ & NAXN (I) & {$[-]$} & {$[-]$} \\
\hline Number of pairs of elements in axial power profile table I & \\
\hline Integer & Required \\
\hline$>0$ & \\
\hline
\end{tabular}

CARD 11.4: $Y(I, N), \operatorname{AXIALZ}(M, I, N) ; N=1: \operatorname{NAXN}(I)$

\begin{tabular}{|l|l|c|c|}
\hline $\mathbf{1 1 . 4}$ & $\mathrm{Y}(\mathrm{I}, \mathrm{N})$ & {$[\mathrm{m}]$} & {$[\mathrm{in}]$} \\
\hline \multicolumn{2}{|l|}{ Vertical location. Note that the axial location should be absolute to the model, not relative to the rod. } \\
\hline Float & Required \\
\hline$>=0$ & \\
\hline
\end{tabular}

\begin{tabular}{|l|l|c|c|}
\hline $\mathbf{1 1 . 4}$ & AXIALZ $(\mathrm{M}, \mathrm{I}, \mathrm{N})$ & {$[-]$} & {$[-]$} \\
\hline Relative axial power factor (ratio of local power to average power) at vertical location $\mathrm{Y}(\mathrm{I}, \mathrm{N})$ \\
\hline Float & Required \\
\hline$>=0$ & \\
\hline
\end{tabular}

\subsection{Total Power Forcing Function}

Card 11.5 is read only if NQ $>0$.

CARD 11.5: $\mathrm{YQ}(\mathrm{N}), \mathrm{FQ}(\mathrm{N}) ; \mathrm{N}=1: \mathrm{NQ}$

\begin{tabular}{|c|c|c|c|}
\hline 11.5 & $\mathrm{YQ}(\mathrm{N})$ & {$[\mathrm{s}]$} & {$[\mathrm{s}]$} \\
\hline \multicolumn{4}{|c|}{ Transient time } \\
\hline Float & \multicolumn{3}{|c|}{ Conditional - if NQ $>0$} \\
\hline$>=0.0$ & & & \\
\hline
\end{tabular}

\begin{tabular}{|c|c|c|c|}
\hline 11.5 & $F Q(N)$ & {$[-]$} & {$[-]$} \\
\hline \multicolumn{4}{|c|}{$\begin{array}{l}\text { Power factor: } \\
\mathrm{FQ}(\mathrm{N})=\frac{\text { power at time } \mathrm{YQ}(\mathrm{N})}{\text { initial power }}\end{array}$} \\
\hline Float & Condi & & \\
\hline
\end{tabular}




\subsection{Gap Conductance Forcing Function}

Card 11.6 is read only if NGPFF $>0$.

CARD 11.6: $\operatorname{YGPFF}(\mathrm{N}), \mathrm{FGPFF}(\mathrm{N}), \mathrm{N}=1$ : NGPFF

\begin{tabular}{|c|c|c|c|}
\hline 11.6 & YGPFF (N) & {$[\mathrm{s}]$} & [s] \\
\hline \multicolumn{4}{|c|}{ Transient time } \\
\hline Float & \multicolumn{3}{|c|}{ Conditional - if NGPFF $>0$} \\
\hline$>=0.0$ & & & \\
\hline
\end{tabular}

\begin{tabular}{|l|l|c|c|}
\hline 11.6 & FGPFF $(\mathrm{N})$ & {$[-]$} & {$[-]$} \\
\hline $\begin{array}{l}\text { Conductance factor: } \\
\text { FGPFF }(\mathrm{N})=\frac{\text { conductance at time YGPFF }(\mathrm{N})}{\text { initial conductance }}\end{array}$ \\
\hline Float & Conditional - if NGPFF $>0$ \\
\hline$>=0.0$ & \\
\hline
\end{tabular}

\subsection{Radial Power Profile Forcing Function}

Cards CARD 11.7 and CARD 11.8 are read in pairs NQR times.

CARD 11.7: YQR

\begin{tabular}{|c|c|c|c|}
\hline 11.7 & YQR & {$[\mathrm{s}]$} & [s] \\
\hline \multicolumn{4}{|c|}{ Transient time for each radial power profile change } \\
\hline Float & Required & & \\
\hline
\end{tabular}

CARD 11.8: FQR (N), N=1:NRROD

\begin{tabular}{|c|c|c|c|}
\hline 11.8 & $\mathrm{FQR}(\mathrm{N})$ & {$[-]$} & {$[-]$} \\
\hline \multicolumn{4}{|c|}{$\begin{array}{l}\text { Radial power factors for all rods (normalized to average power) starting from rod number } 1 \text {. Skipping } \\
\text { rod numbers is not permitted. } \\
\text { Note: Eight values are entered per card. If NRROD is greater than } 8 \text {, repeat CARD } 11.8 \text { until NRROD } \\
\text { values for FQR have been entered. }\end{array}$} \\
\hline Float & Required & & \\
\hline$>0.0$ & & & \\
\hline
\end{tabular}




\section{Card Group 12}

This card group is read by subroutine SETIN.

The first line indicates the group number: NGROUP $=12$

CARD 12.1 is read if IMIX $=1$ (See Card 1.1)

CARD 12.1: AAAK, BETA

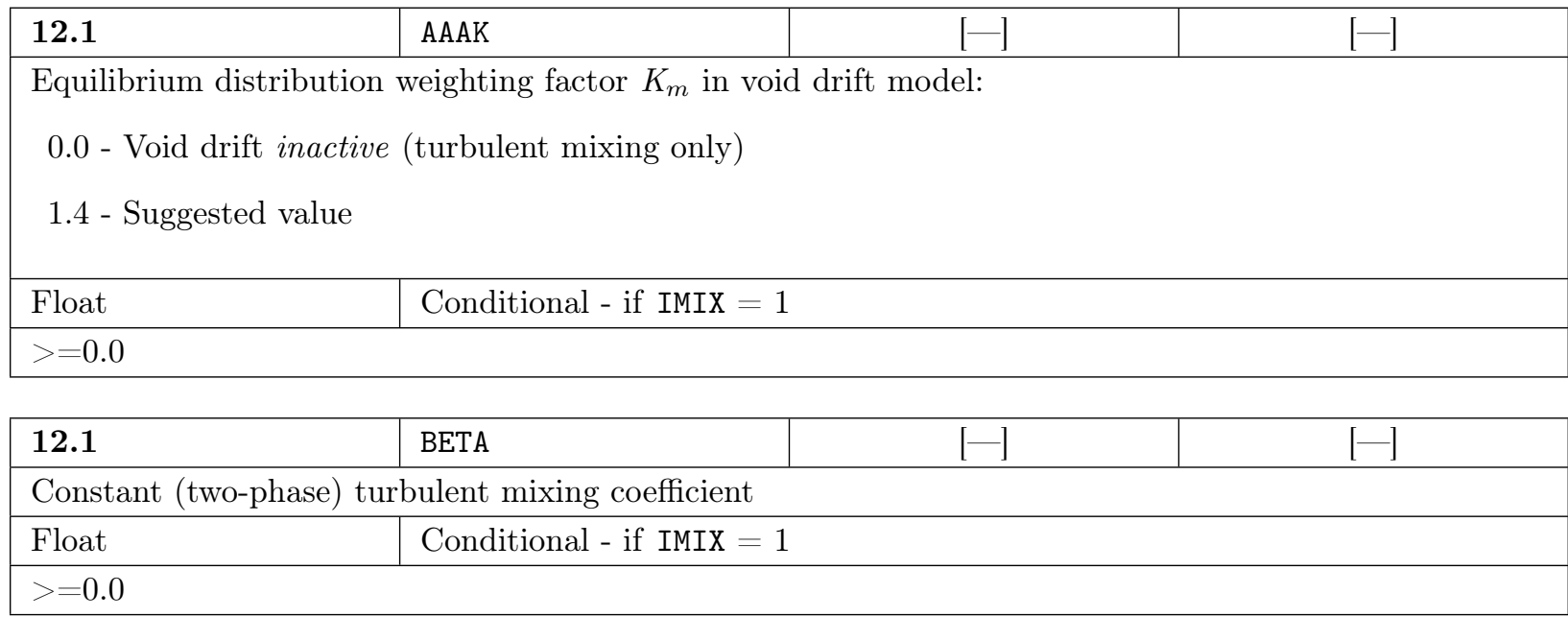

CARD 12.2 is read if IMIX $=2($ See Card 1.1$)$

CARD 12.2: AAAK, DFROD, THETM 


\begin{tabular}{|c|c|c|c|}
\hline 12.2 & AAAK & {$[-]$} & {$[-]$} \\
\hline \multicolumn{4}{|c|}{ Equilibrium distribution weighting factor $K_{m}$ in void drift model: } \\
\hline \multicolumn{4}{|c|}{0.0 - Void drift inactive (turbulent mixing only) } \\
\hline \multicolumn{4}{|c|}{1.4 - Suggested value } \\
\hline Float & Condi & & \\
\hline$>=0.0$ & & & \\
\hline
\end{tabular}

\begin{tabular}{|c|c|c|c|}
\hline 12.2 & DFROD & [m] & [in] \\
\hline \multicolumn{4}{|c|}{ Outside rod diameter - must be consistent with DROD in CARD 9.2 or CARD 9.6! } \\
\hline Float & Condit & & \\
\hline \multicolumn{4}{|l|}{$>0.0$} \\
\hline
\end{tabular}

\begin{tabular}{|l|l|c|c|}
\hline $\mathbf{1 2 . 2}$ & THETM & {$[-]$} & {$[-]$} \\
\hline
\end{tabular}

Ratio between the maximum two-phase turbulent mixing coefficient (near the transition between slug and annular flow) and the single-phase mixing coefficient (in single-phase liquid). Suggested value is given by Beus to be 5.0 [2].

\begin{tabular}{|l|l|}
\hline Float & Conditional - if IMIX $=2$ \\
\hline$>=0.0$ & \\
\hline
\end{tabular}

CARD 12.3 is read if IMIX $=3($ See Card 1.1$)$

CARD 12.3: AAAK, BETA, THETM

\begin{tabular}{|c|c|c|c|}
\hline 12.3 & AAAK & {$[-]$} & {$[-]$} \\
\hline \multicolumn{4}{|c|}{ Equilibrium distribution weighting factor $K_{m}$ in void drift model: } \\
\hline \multicolumn{4}{|c|}{0.0 - Void drift inactive (turbulent mixing only) } \\
\hline \multicolumn{4}{|c|}{1.4 - Suggested value } \\
\hline Float & \multicolumn{3}{|c|}{ Conditional - if IMIX $=3$} \\
\hline \multicolumn{4}{|c|}{$>=0.0$} \\
\hline 12.3 & BETA & {$[-]$} & {$[-]$} \\
\hline \multicolumn{4}{|c|}{$\begin{array}{l}\text { Constant single-phase turbulent mixing coefficient. Beus will be used to set the two-phase mixing } \\
\text { coefficient from the single-phase mixing coefficient. }\end{array}$} \\
\hline Float & \multicolumn{3}{|c|}{ Conditional - if IMIX $=3$} \\
\hline \multicolumn{4}{|c|}{$>=0.0$} \\
\hline
\end{tabular}




\begin{tabular}{|c|c|c|c|}
\hline 12.3 & THETM & {$[-]$} & {$[-]$} \\
\hline \multicolumn{4}{|c|}{$\begin{array}{l}\text { Ratio between the maximum two-phase turbulent mixing coefficient (near the transition between slug } \\
\text { and annular flow) and the single-phase mixing coefficient (in single-phase liquid). Suggested value is } \\
\text { given by Beus to be } 5.0 \text {. }\end{array}$} \\
\hline Float & Condit & & \\
\hline
\end{tabular}




\section{Card Group 13}

This card group is read by subroutine READ_CARD_13.

The first line indicates the group number: NGROUP $=13$

CARD 13.1: NIBND, NKBND, NFNUCT, NGBND, NIBNDB, BCVEL, NDUM7, NDUM8, NDUM9, NDUM10, NDUM11, NDUM12, NDUM13, NDUM14

\begin{tabular}{|l|l|c|c|}
\hline $\mathbf{1 3 . 1}$ & NIBND & {$[-]$} & {$[-]$} \\
\hline \multicolumn{2}{|l|}{ Total number of vertical mesh cell boundary conditions. Includes inlet, outlet, and internal mesh cells. } \\
\hline Integer & Required \\
\hline$>=0$ & \\
\hline
\end{tabular}

\begin{tabular}{|c|c|c|c|}
\hline 13.1 & NKBND & {$[-]$} & {$[-]$} \\
\hline \multicolumn{4}{|c|}{ Total number of transverse mesh cells for which cross-flow will be set to zero } \\
\hline Integer & Requir & & \\
\hline \multicolumn{4}{|l|}{$>=0$} \\
\hline
\end{tabular}

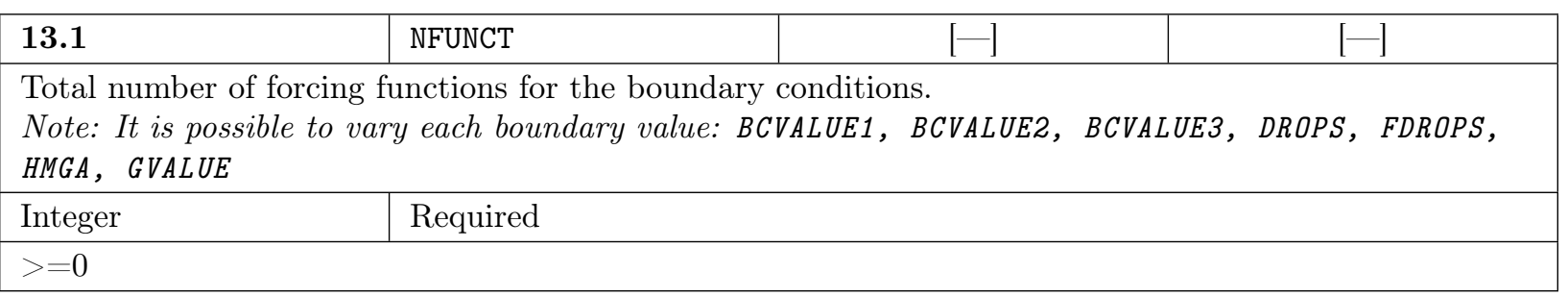

\begin{tabular}{|l|l|c|c|}
\hline $\mathbf{1 3 . 1}$ & NGBND & {$[-]$} & {$[-]$} \\
\hline Number of groups of adjacent transverse momentum cells for which cross-flow will be set to zero \\
\hline Integer & Required \\
\hline$>=0$ & \\
\hline
\end{tabular}




\begin{tabular}{|l|l|c|}
\hline $\mathbf{1 3 . 1}$ & NIBNDB & {$[-]$} \\
\hline $\begin{array}{l}\text { Total number of vertical mesh cell boundary conditions to which the boron tracking/precipitation model } \\
\text { is applied. This is the number of inlet boron boundary conditions. }\end{array}$ \\
\hline Integer & Required \\
\hline$>=0$ &
\end{tabular}

\begin{tabular}{|l|l|c|}
\hline $\mathbf{1 3 . 1}$ & BCVEL & {$[-]$} \\
\hline $\begin{array}{l}\text { Set to 1 if you would like to change the meaning of BCVALUE1 (specified on Card 13.4) to mean } \\
\text { velocity instead of mass flow rate. }\end{array}$ \\
\hline Integer & Required \\
\hline 0,1 & \\
\hline
\end{tabular}

\begin{tabular}{l|l|c|c|}
\hline $\mathbf{1 3 . 1}$ & NDUM7:NDUM14 & {$[-]$} & {$[-]$} \\
\hline $\begin{array}{l}\text { Not used, but entry is obligatory: } \\
0 \text { - Suggested value }\end{array}$ \\
\hline \begin{tabular}{l} 
Integer \\
\hline 0
\end{tabular}
\end{tabular}

CARD 13.2 is read only if NFUNCT $>0$

CARD 13.2: $\operatorname{NPTS}(K) ; K=1:$ NFUNCT

\begin{tabular}{|l|l|c|c|}
\hline $\mathbf{1 3 . 2}$ & NPTS $(\mathrm{K})$ & {$[-]$} & {$[-]$} \\
\hline $\begin{array}{l}\text { Number of pairs of values in forcing function table K } \\
16 \text { values are entered per card line. Repeat CARD } 13.2 \text { until NFUNCT values have been entered. }\end{array}$ \\
\hline Integer & Conditional - if NFUNCT $>0$ \\
\hline$>0$ & \\
\hline
\end{tabular}

CARD 13.3 is read NFUNCT times

CARD 13.3: $\operatorname{ABSCIS}(K, I), \operatorname{ORDINT}(K, I) ; I=1: \operatorname{NPTS}(K) ; K=1: \operatorname{NFUNCT}$

\begin{tabular}{|c|c|c|c|}
\hline 13.3 & $\operatorname{ABSCIS}(K, I)$ & {$[\mathrm{s}]$} & {$[\mathrm{s}]$} \\
\hline \multicolumn{4}{|c|}{ Transient time } \\
\hline Float & Required & & \\
\hline$>=0.0$ & & & \\
\hline
\end{tabular}




\begin{tabular}{|c|c|c|c|}
\hline 13.3 & $\operatorname{ORDINT}(\mathrm{K}, \mathrm{I})$ & {$[-]$} & {$[-]$} \\
\hline \multicolumn{4}{|c|}{$\begin{array}{l}\text { Forcing function factor to be applied at time ABSCIS (K, I) } \\
\text { Five pairs of (ABSCIS, ORDINT) are entered per card. Repeat CARD } 13.3 \text { until NPTS (K) points have } \\
\text { been entered for forcing function table K. Continue entering data until NFUNCT tables have been specified. }\end{array}$} \\
\hline Float & Required & & \\
\hline
\end{tabular}

CARD 13.4, CARD 13.5, CARD 13.6, and CARD 13.7 are read NIBND times.

CARD 13.4: IBOUND $(\mathrm{L}, \mathrm{N}), \operatorname{ISPEC}(\mathrm{N}), \operatorname{N1FN}(\mathrm{N}), \operatorname{N2FN}(\mathrm{N}), \mathrm{N} 3 F N(\mathrm{~N}), \operatorname{BCVALUE} 1(\mathrm{~N}), \operatorname{BCVALUE2}(\mathrm{N}), \operatorname{BCVALUE3}(\mathrm{N})$, INITGAS; $\mathrm{L}=1: 2, \mathrm{~N}=1: \mathrm{NIBND}$

\begin{tabular}{|l|l|l|}
\hline $\mathbf{1 3 . 4}$ & $\operatorname{IBOUND}(1, \mathrm{~N})$ & {$[-]$} \\
\hline Index number of the channel at which boundary condition N is applied \\
\hline Integer & Required \\
\hline Must be channel index defined in model \\
\hline
\end{tabular}

\begin{tabular}{|l|l|l|}
\hline $\mathbf{1 3 . 4}$ & $\operatorname{IBOUND}(2, \mathrm{~N})$ & {$[-]$} \\
\hline Vertical node number at which boundary condition $\mathrm{N}$ is applied. \\
Note: The node number counts relative to the first node of the section in which the channel identified \\
in IBOUND $(1, N)$ resides. the auxiliary (boundary) cells are counted as well. \\
For example: 1 section with NONODE $=80$ continuity mesh cells: \\
Inlet: IBOUND $(2, \mathrm{~N})=1$ \\
Outlet: IBOUND $(2, \mathrm{~N})=82$ \\
\hline Integer & Required \\
\hline$>0$ and $<=$ number of levels in channel \\
\hline
\end{tabular}

\begin{tabular}{|c|c|c|c|}
\hline 13.4 & $\operatorname{ISPEC}(\mathrm{N})$ & {$[-]$} & {$[-]$} \\
\hline \multicolumn{4}{|c|}{ Boundary condition type: } \\
\hline \multicolumn{4}{|c|}{1 - pressure and enthalpy (or temperature) } \\
\hline \multicolumn{4}{|c|}{2 - mass flow rate and enthalpy (or temperature) } \\
\hline \multicolumn{4}{|c|}{3 - mass flow rate only } \\
\hline \multicolumn{4}{|c|}{4 - mass source (mass flow rate and enthalpy) } \\
\hline \multicolumn{4}{|c|}{5 - pressure sink (pressure and enthalpy) } \\
\hline \multicolumn{4}{|c|}{6 - mass flow rate, void, and slip ratio (only works for inlet) } \\
\hline Integer & Required & & \\
\hline - & & & \\
\hline
\end{tabular}




\begin{tabular}{|l|c|c|}
\hline $\mathbf{1 3 . 4}$ & $\mathrm{N} 1 \mathrm{FN}(\mathrm{N})$ & {$[-]$} \\
\hline Index number of the forcing function table by which the first parameter of the boundary condition \\
(BCVALUE1) will be varied \\
0 - if the boundary condition is constant \\
Note: The forcing function tables are numbered sequentially in the order they are read in CARD 13.3 \\
For example: If ISPEC (N) = 3 and N1FN (N) =3, the specified mass flow rate will be adjusted according \\
to the 3rd forcing function table entered in CARD 13.3 \\
\hline Integer \\
\hline Must be forcing function index defined in model \\
\hline
\end{tabular}

\begin{tabular}{|c|c|c|c|}
\hline 13.4 & $\mathrm{~N} 2 \mathrm{FN}(\mathrm{N})$ & {$[-]$} & {$[-]$} \\
\hline \multicolumn{4}{|c|}{$\begin{array}{l}\text { Index number of the forcing function table by which the second parameter of the boundary condition } \\
\text { (BCVALUE2) will be varied }\end{array}$} \\
\hline \multicolumn{4}{|c|}{0 - if the boundary condition is constant } \\
\hline \multicolumn{4}{|c|}{$\begin{array}{l}\text { For example: If ISPEC }(\mathrm{N})=1 \text { and } \mathrm{N} 2 \mathrm{FN}(\mathrm{N})=6 \text {, the specified enthalpy will be adjusted according to } \\
\text { the 6th forcing function table entered in CARD } 13.3\end{array}$} \\
\hline Integer & Required & & \\
\hline
\end{tabular}

\begin{tabular}{|c|c|c|c|}
\hline 13.4 & N3FN $(N)$ & {$[-]$} & {$[-]$} \\
\hline \multicolumn{4}{|c|}{$\begin{array}{l}\text { Index number of the forcing function table by which the third parameter of the boundary condition } \\
\text { (BCVALUE3) will be varied }\end{array}$} \\
\hline \multicolumn{4}{|c|}{0 - if the boundary condition is constant } \\
\hline Integer & Required & & \\
\hline
\end{tabular}

\begin{tabular}{|c|c|c|c|}
\hline 13.4 & BCVALUE1 (N) & {$[\mathrm{kg} / \mathrm{s}$ or $\mathrm{m} / \mathrm{s}]$} & {$[\mathrm{lbm} / \mathrm{s}$ or $\mathrm{ft} / \mathrm{s}]$} \\
\hline \multicolumn{4}{|c|}{$\begin{array}{l}\text { Mass flow rate boundary condition if } \mathrm{BCVEL}=0 \text { (Card } 13.1) \text { or velocity boundary condition } \\
\mathrm{BCVEL}=1 \text {. Only valid for certain ISPEC values. Enter if } \operatorname{ISPEC}(\mathrm{N})=2,3,4 \text {, or } 6 \text {. Has no effect } \\
\text { if } \operatorname{ISPEC}(\mathrm{N})=1 \text { or } 5 \text {. }\end{array}$} \\
\hline Float & Required & & \\
\hline Any & & & \\
\hline
\end{tabular}




\begin{tabular}{|c|c|c|c|}
\hline 13.4 & BCVALUE2 (N) & {$\left[\mathrm{kJ} / \mathrm{kg} \underset{\text { or }}{ }{ }^{\circ} \mathrm{C}\right.$ its $\underline{\text { or }}$ no } & $\underset{\text { units }]}{[\mathrm{BTU} / \mathrm{lbm} \underset{\text { or }}{ }}{ }^{\circ} \mathrm{F}$ or no \\
\hline \multicolumn{4}{|c|}{ Second boundary condition value: } \\
\hline \multicolumn{4}{|c|}{$\begin{array}{l}\text { Enthalpy - if } \operatorname{ISPEC}(\mathrm{N})=1,2,4 \text {, or } 5 \\
\quad \underline{\text { or }}\end{array}$} \\
\hline \multicolumn{4}{|c|}{$\begin{array}{l}\text { Temperature - enter as a negative of the number } \\
\text { Example: for } 300^{\circ} \mathrm{C} \text {, enter }-300.0\end{array}$} \\
\hline \multicolumn{4}{|c|}{$0.0-$ if $\operatorname{ISPEC}(\mathrm{N})=3$} \\
\hline \multicolumn{4}{|c|}{ Void - if $\operatorname{ISPEC}(\mathrm{N})=6$} \\
\hline Float & Required & & \\
\hline - & & & \\
\hline
\end{tabular}

\begin{tabular}{|c|c|c|c|}
\hline 13.4 & BCVALUE3 $(\mathrm{N})$ & [bar or no units] & [psia or no units] \\
\hline \multicolumn{4}{|c|}{ Third boundary condition value: } \\
\hline \multicolumn{4}{|c|}{ Pressure - if $\operatorname{ISPEC}(\mathrm{N})=1,4$, or 5} \\
\hline \multicolumn{4}{|c|}{$0.0-$ if $\operatorname{ISPEC}(\mathrm{N})=2$ or 3} \\
\hline \multicolumn{4}{|c|}{ Slip ratio - if $\operatorname{ISPEC}(\mathrm{N})=6$} \\
\hline \multicolumn{4}{|c|}{$\begin{array}{l}\text { Note: The enthalpy specified at the exit is not used by COBRA-TF if flow is in the positive direction } \\
\text { (i.e. out of the model). In the case of positive flow, the user may enter any number for exit enthalpy }\end{array}$} \\
\hline Float & Required & & \\
\hline
\end{tabular}

\begin{tabular}{|c|c|c|c|}
\hline 13.4 & INITGAS & {$[-]$} & {$[-]$} \\
\hline \multicolumn{4}{|c|}{$\begin{array}{l}\text { Flag for the inlet boundary conditions of non-condensable gases (only relevant in the case that ISPEC }(\mathrm{N}) \\
\neq 3) \text { : }\end{array}$} \\
\hline \multicolumn{4}{|c|}{0 - Inlet conditions will be entered in CARD 13.6 and CARD 13.7} \\
\hline \multicolumn{4}{|c|}{$\begin{array}{l}1 \text { - Inlet conditions will be set equal to the initial conditions entered in CARD } 1.3 \text { and CARD } 1.4 \\
\text { with the following assumptions: } \\
\text { HMGA = HGIN } \\
\text { GVALUE = VFRAC } \\
\text { NHMFN }=0 \\
\text { NGFN }=0\end{array}$} \\
\hline Integer & Required & & \\
\hline$>=0$ & & & \\
\hline
\end{tabular}


CARD 13.5 is only read if $\operatorname{ISPEC}(\mathrm{N})=4$ (mass injection boundary condition)

CARD 13.5: $\operatorname{DROPS}(\mathrm{N}), \operatorname{NDFN}(\mathrm{N}), \operatorname{FDROPS}(\mathrm{N}), \operatorname{NDFFN}(\mathrm{N})$

\begin{tabular}{|l|l|c|c|}
\hline $\mathbf{1 3 . 5}$ & DROPS $(\mathrm{N})$ & {$[\mathrm{m}]$} & [in] \\
\hline Droplet diameter & Conditional - if ISPEC $(\mathrm{N})=4$ \\
\hline Float & \\
\hline$>=0.0$ & \\
\hline
\end{tabular}

\begin{tabular}{|l|l|c|c|}
\hline $\mathbf{1 3 . 5}$ & NDFN $(\mathrm{N})$ & {$[-]$} & {$[-]$} \\
\hline Index number of the forcing function table by which the DROPS (N) parameter is varied \\
\hline Integer & Conditional - if ISPEC (N) $=4$ \\
\hline Must be forcing function index defined in model \\
\hline
\end{tabular}

\begin{tabular}{|l|l|l|l|}
\hline $\mathbf{1 3 . 5}$ & FDROPS $(\mathrm{N})$ & {$[\mathrm{kg} / \mathrm{s}]$} & {$[\mathrm{lbm} / \mathrm{s}]$} \\
\hline \multicolumn{2}{|l|}{} & \\
\hline Droplet mass flow rate at injection boundary & Conditional - if ISPEC (N) $=4$ \\
\hline Any & \\
\hline
\end{tabular}

\begin{tabular}{|l|l|c|c|}
\hline $\mathbf{1 3 . 5}$ & NDFFN (N) & {$[-]$} & {$[-]$} \\
\hline Index number of the forcing function table by which the FDROPS (N) parameter is varied \\
\hline Integer & Conditional - if ISPEC (N) $=4$ \\
\hline Must be forcing function index defined in model \\
\hline
\end{tabular}

CARD 13.6 and CARD 13.7 are only read if $\operatorname{ISPEC}(\mathrm{N}) \neq 3$ and INITGAS $=0$. The structure of variables in CARD 13.6 is similar to that in CARD 1.3 and CARD 1.4, where enthalpy and void fractions are specified as initial conditions. (These initial values can be overridden by setting INITGAS $=1$, see CARD 13.4)

CARD 13.6: HMGA (N), GVALUE, (NGA,N); NGA=1:NGAS+2

\begin{tabular}{|c|c|c|c|}
\hline 13.6 & HMGA (N) & {$[\mathrm{kJ} / \mathrm{kg}]$} & {$[\mathrm{BTU} / \mathrm{lbm}]$} \\
\hline \multicolumn{4}{|c|}{ Enthalpy of non-condensable gas mixture } \\
\hline Float & \multicolumn{3}{|c|}{ Conditional - if $\operatorname{ISPEC}(\mathrm{N}) \neq 3$ and INITGAS $=0$} \\
\hline$>0.0$ & & & \\
\hline
\end{tabular}




\begin{tabular}{|l|l|l|}
\hline 13.6 & GVALUE $(\mathrm{NGA}, \mathrm{N})$ & {$[-]$} \\
\hline Volume fractions (inlet boundary condition) of: & {$[-]$} \\
NGA $=1$ - liquid in the liquid-vapor-gas mixture \\
NGA $=2$ - vapor in the vapor-gas mixture \\
NGA $\geq 3$ - gas (NGA - 2) in the vapor-gas mixture \\
\hline Float & Conditional - if ISPEC $(\mathrm{N}) \neq 3$ and INITGAS $=0$ \\
\hline$>=0.0$ and $<=1.0$ & \\
\hline
\end{tabular}

CARD 13.7: $\operatorname{NHMFN}(\mathrm{N}), \operatorname{NGFN}(\mathrm{NGA}, \mathrm{N}) ; \mathrm{NGA}=1: \mathrm{NGAS}+2$

\begin{tabular}{|l|l|c|}
\hline $\mathbf{1 3 . 7}$ & $\operatorname{NHMFN}(\mathrm{N})$ & {$[-]$} \\
\hline $\begin{array}{l}\text { Index number of forcing function applied to the enthalpy of non-condensable gas mixture HMGA (see } \\
\text { CARD 13.6) }\end{array}$ \\
\hline Integer & Conditional - if ISPEC $(\mathrm{N}) \neq 3$ and INITGAS $=0$ \\
\hline Must be forcing function index defined in model \\
\hline
\end{tabular}

\begin{tabular}{|l|l|c|c|}
\hline $\mathbf{1 3 . 7}$ & NGFN $(\mathrm{NGA}, \mathrm{N})$ & {$[-]$} & {$[-]$} \\
\hline Index number of the forcing function applied to the volume fractions GVALUE (see CARD 13.6) \\
\hline Integer & Conditional - if ISPEC $(\mathrm{N}) \neq 3$ and INITGAS $=0$ \\
\hline Must be forcing function index defined in model \\
\hline
\end{tabular}

CARD 13.8 and CARD 13.9 are read in pairs NIBND times

CARD 13.8 is only read if $\operatorname{ISPEC}(\mathrm{N})=4$ (mass injection boundary condition)

CARD 13.8: $\operatorname{AINJT}(\mathrm{K})$

$\mathrm{K}$ is an integer corresponding to the total number of vertical boundary conditons cells at which boundary condition type 4 is specified. $\mathrm{K}=1,2, \ldots$, total number of vertical boundary cells.

\begin{tabular}{|c|c|c|c|}
\hline 13.8 & $\operatorname{AINJT}(\mathrm{K})$ & {$\left[\mathrm{m}^{2}\right]$} & {$\left[\mathrm{in}^{2}\right]$} \\
\hline \multicolumn{4}{|c|}{ Flow area of the mass injection } \\
\hline Float & Condition & & \\
\hline
\end{tabular}

CARD 13.9 is only read if $\operatorname{ISPEC}(\mathrm{N})=5$ (pressure sink boundary condition)

CARD 13.9: ASINK (K), SINKK (K), DXSINK (K)

$\mathrm{K}$ is an integer corresponding to the total number of vertical boundary conditons cells at which boundary condition type 5 is specified. $\mathrm{K}=1,2, \ldots$, total number of vertical boundary cells. 


\begin{tabular}{|l|l|c|c|}
\hline $\mathbf{1 3 . 9}$ & $\operatorname{ASINK}(\mathrm{K})$ & {$\left[\mathrm{m}^{2}\right]$} & {$\left[\mathrm{in}^{2}\right]$} \\
\hline \multicolumn{2}{|l|}{} \\
\hline Flow area of the pressure $\operatorname{sink}$ & Conditional - if $\operatorname{ISPEC}(\mathrm{N})=5$ \\
\hline$>0.0$ & \\
\hline
\end{tabular}

\begin{tabular}{|l|l|l|l|}
\hline $\mathbf{1 3 . 9}$ & SINKK (K) & {$[-]$} & {$[-]$} \\
\hline Pressure loss coefficient (velocity head) of the pressure sink & \\
\hline Float & Conditional - if ISPEC (N) $=5$ \\
\hline$>=0.0$ & \\
\hline
\end{tabular}

\begin{tabular}{|l|l|l|l|}
\hline $\mathbf{1 3 . 9}$ & DXSINK (K) & {$[\mathrm{m}]$} & [in] \\
\hline \multicolumn{2}{|l|}{ Length of the momentum } & & \\
\hline Float & Control volume for the sink & \\
\hline$>0.0$ & \\
\hline
\end{tabular}

CARD 13.10 is read NGBND times (only if NGBND > 0). This means that CARD 13.10 may be repeated as many times as necessary for a given gap $\mathrm{K}$ in order to identify all axial levels that have zero cross flow. The total number of transverse momentum cells with zero cross flow boundary conditions specified by CARD 13.10 must sum to NKBND.

CARD 13.10: K, JSTART, JEND

\begin{tabular}{|l|l|l|l|}
\hline $\mathbf{1 3 . 1 0}$ & $\mathrm{K}$ & {$[-]$} & {$[-]$} \\
\hline Gap number to which a zero cross flow is to be applied \\
\hline Integer & Conditional - if NGBND >0 \\
\hline Must be a gap index declared in model \\
\hline
\end{tabular}

\begin{tabular}{|c|c|c|c|}
\hline 13.10 & JSTART & {$[-]$} & {$[-]$} \\
\hline \multicolumn{4}{|c|}{$\begin{array}{l}\text { Continuity cell number at which to start applying the zero cross flow } \\
\text { Note: The cross flow will be set to zero for gap K between nodes JSTART and JEND. The node numbers } \\
\text { are given relative to the beginning of the section containing gap K. }\end{array}$} \\
\hline Integer & Conditi & & \\
\hline \multicolumn{4}{|l|}{-} \\
\hline
\end{tabular}

\begin{tabular}{|l|l|c|}
\hline $\mathbf{1 3 . 1 0}$ & JEND & {$[-]$} \\
\hline $\begin{array}{l}\text { Continuity cell number at which to stop applying the zero cross flow } \\
\text { Note: The cross flow will be set to zero for gap K between nodes JSTART and JEND. The node numbers } \\
\text { are given relative to the beginning of the section containing gap K. }\end{array}$ \\
\hline Integer & Conditional - if NGBND >0 \\
\hline-
\end{tabular}

CARD 13.11 is read NIBNDB times (boron inlet boundary conditions) if IBTM of CARD $1.1 \neq 0$ 
Note: Variable NIBNDB, as the number of boron inlet B.C., does not need to match with the number of inlet B.C. entered in CARD 13.4

Note: There must be a match between where the inlet B.C. and the boron inlet B.C. are applied; (IBOUND (L , N), $L=1,2)$ and $(\operatorname{IBOUNDB}(L, N) ; L=1: 2)$

Note: The boron tracking/precipitation model is applied when ISPEC $(N)=1$, 2 or 3 . The variables related with boron inlet B.C., N4FNB(N) and BCVALUE4B(N), are reassigned as extra inlet B.C. as N4FN(N) and $B C V A L U E 4(N) ; N=1: N I B N D$

13.11: $\operatorname{IBOUNDB}(\mathrm{L}, \mathrm{N}), \operatorname{N4FNB}(\mathrm{N}), \operatorname{BCVALUE} 4 B(\mathrm{~N}) ; \mathrm{L}=1: 2, \mathrm{~N}=1: \mathrm{NIBNDB}$

\begin{tabular}{|l|l|l|}
\hline $\mathbf{1 3 . 1 1}$ & $\operatorname{IBOUNDB}(1, \mathrm{~N})$ & {$[-]$} \\
\hline Index number of the channel at which boundary condition $\mathrm{N}$ is applied \\
\hline Integer & Conditional - if IBTM $>0$ \\
\hline Must be a channel index declared in model \\
\hline
\end{tabular}

\begin{tabular}{|c|c|c|c|}
\hline 13.11 & $\operatorname{IBOUNDB}(2, \mathrm{~N})$ & {$[-]$} & {$[-]$} \\
\hline \multicolumn{4}{|c|}{$\begin{array}{l}\text { Vertical node number at which boundary condition } \mathrm{N} \text { is applied } \\
\text { Note: The node number counts relatively to the first node of the section in which the channel identified } \\
\text { in IBOUND }(1, N) \text { resides. The auxiliary (boundary) cells are counted, too } \\
\text { Example: } 1 \text { section with NONODE }=80 \text { continuity mesh cells: } \\
\text { Inlet: } \operatorname{IBOUND}(2, \mathrm{~N})=1 \text { Outlet: } \operatorname{IBOUND}(2, \mathrm{~N})=82\end{array}$} \\
\hline Integer & Conditional - if & & \\
\hline
\end{tabular}

13.11 $\quad$ N4FNB $(\mathrm{N})$
Index number of the forcing function table by which the fourth parameter of the boundary condition
(BCVALUE4) is varied:
0 - if the boundary condition is constant
Note: The forcing function tables are numbered sequentially in the order they are read in CARD 13.3
For example: If N4FN (N) $=5$, the specified boron concentration will be adjusted according to the 5th
forcing function table entered in CARD 13.3.
Integer
Must be a forcing function index declared in model (or zero)

\begin{tabular}{|l|l|l|}
\hline $\mathbf{1 3 . 1 1}$ & BCVALUE4B $(\mathrm{N})$ & {$[\mathrm{ppm}]$} \\
\hline Fourth boundary condition value - boron concentration & {$[\mathrm{ppm}]$} \\
\hline Float & Conditional - if IBTM $>0$ \\
\hline$>=0.0$ & \\
\hline
\end{tabular}




\section{Card Group 14}

There are two options for specifying the code output options. The first option is the legacy Card Group 14. The second option is a new, more intuitive Card Group 14. The legacy Card Group 14 affords the user some options that the new one does not (such as specifying specific channels/rods to print out), but it is also more confusing to use and will not be supported going forward (e.g., one cannot turn the HDF5 file on/off from the legacy Card Group 14). These two options are described in this chapter. Only enter one of these two Card Groups 14s in the input deck. CTF code output is further described in Chapter 22.

\subsection{Legacy Card Group 14}

This card group is read by subroutine READ_CARD_14.

The first line indicates the group number: NGROUP $=14$

CARD 14.1: N1, NOUT1, NOUT2, NOUT3, NOUT4, IPROPP, IOPT, NDUM8, NDUM9, NDUM10, NDUM11, NDUM12, NDUM13, NDUM14 


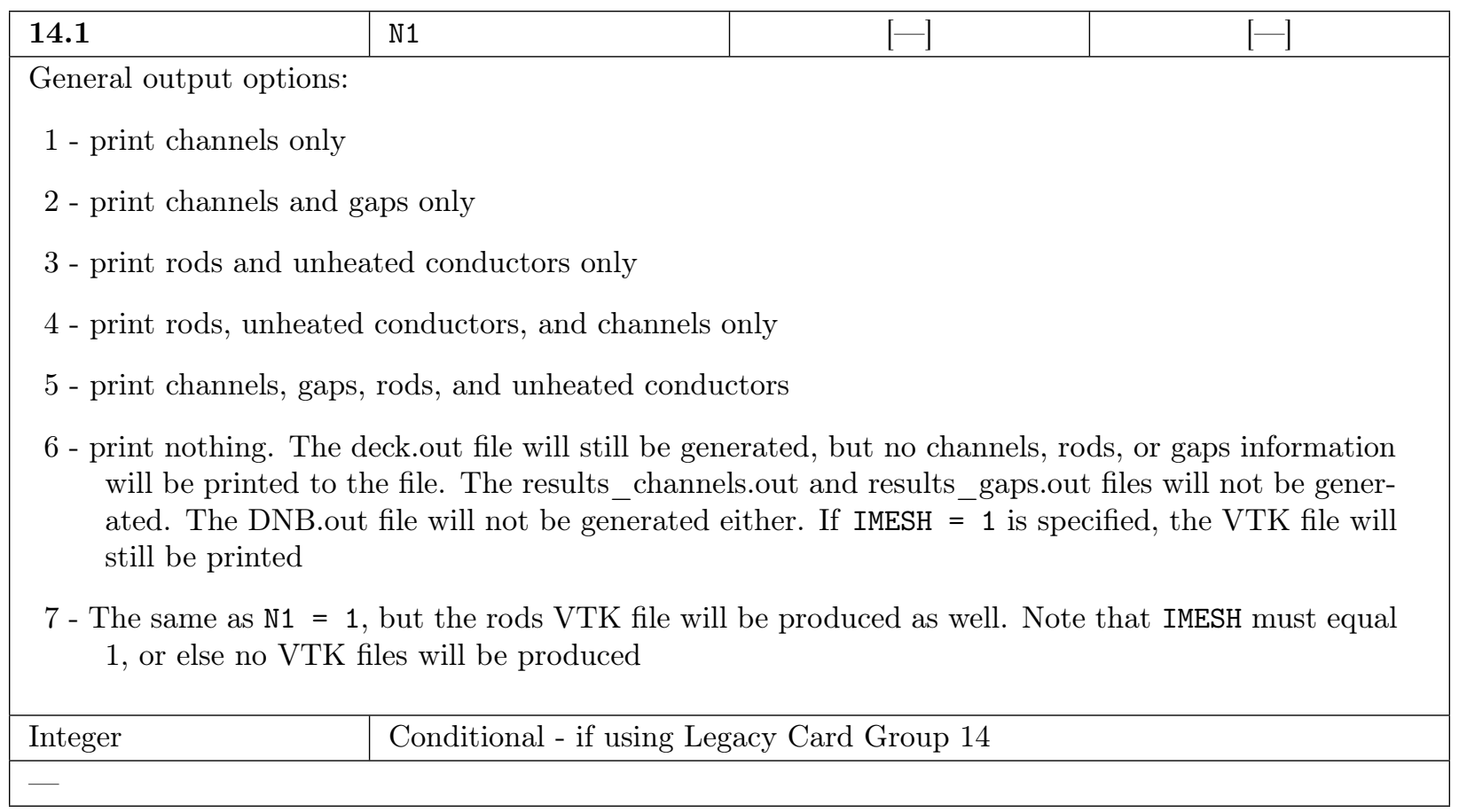

\begin{tabular}{|c|c|c|c|}
\hline 14.1 & NOUT1 & {$[-]$} & {$[-]$} \\
\hline \multicolumn{4}{|c|}{ Number of channels to be printed (only if $\mathrm{N} 1 \neq 3$ ): } \\
\hline \multicolumn{4}{|c|}{0 - all channels will be printed } \\
\hline \multicolumn{4}{|c|}{$>0$ - an array of NOUT1 channel numbers must be entered on CARD 14.2} \\
\hline Integer & Condi & Grou & \\
\hline - & & & \\
\hline
\end{tabular}

\begin{tabular}{|c|c|c|c|}
\hline 14.1 & NOUT2 & [-] & {$[-]$} \\
\hline \multicolumn{4}{|c|}{ Number of rods to be printed (only if $\mathrm{N} 1>2$ ): } \\
\hline \multicolumn{4}{|c|}{0 - all rods will be printed } \\
\hline \multicolumn{4}{|c|}{$>0-$ an array of NOUT2 rod numbers must be entered on CARD 14.4} \\
\hline Integer & Condit & Grou & \\
\hline — & & & \\
\hline
\end{tabular}




\begin{tabular}{|c|c|c|c|}
\hline 14.1 & NOUT3 & {$[-]$} & {$[-]$} \\
\hline \multicolumn{4}{|c|}{ Number of gaps to be printed (only if $\mathrm{N} 1=2$ or 5 ): } \\
\hline \multicolumn{4}{|c|}{0 - all gaps will be printed } \\
\hline \multicolumn{4}{|c|}{$>0$ - an array of NOUT3 gap numbers must be entered on CARD 14.3} \\
\hline Integer & Condit & Grou & \\
\hline - & & & \\
\hline
\end{tabular}

\begin{tabular}{|c|c|c|c|}
\hline 14.1 & NOUT4 & {$[-]$} & {$[-]$} \\
\hline \multicolumn{4}{|c|}{ Number of unheated conductors to be printed (only if $\mathrm{N} 1>2$ ): } \\
\hline \multicolumn{4}{|c|}{0 - all unheated conductors will be printed } \\
\hline \multicolumn{4}{|c|}{$>0$ - an array of NOUT4 unheated conductor numbers must be entered on CARD 14.5} \\
\hline Integer & Condi & Groul & \\
\hline - & & & \\
\hline
\end{tabular}

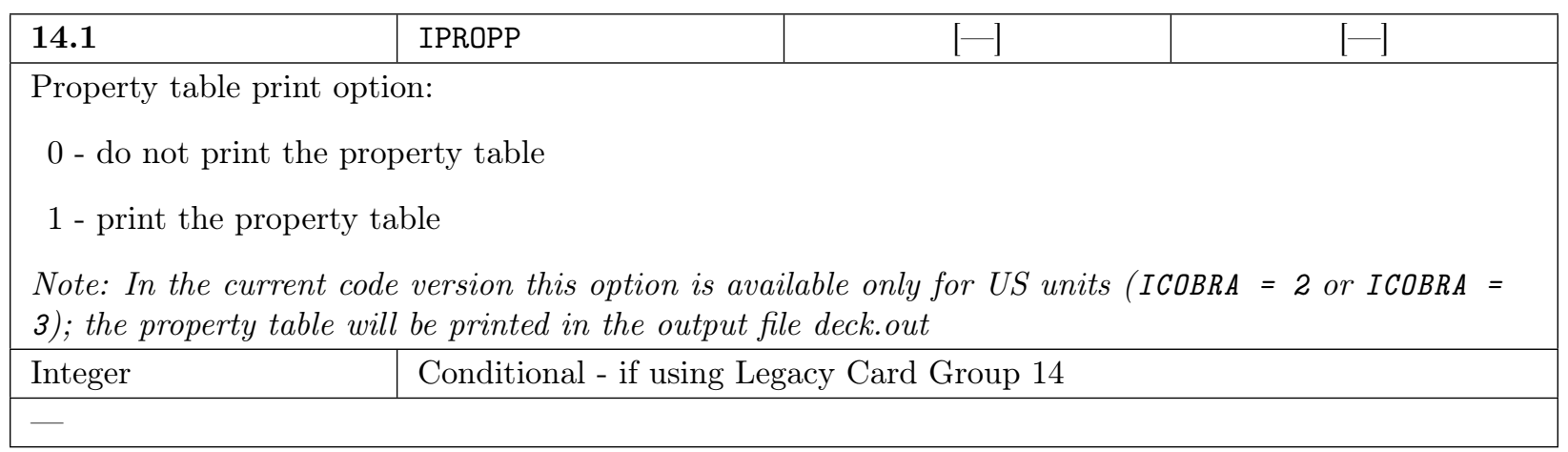

\begin{tabular}{|c|c|c|c|}
\hline 14.1 & IOPT & {$[-]$} & {$[-]$} \\
\hline \multicolumn{4}{|c|}{ Debug print option: } \\
\hline \multicolumn{4}{|c|}{0 - normal printout only } \\
\hline \multicolumn{4}{|c|}{$\begin{array}{l}2 \text { - debug printout (print extra data for subchannels in file results_channels.out, print extra files } \\
\text { time.out and krysolv.out) }\end{array}$} \\
\hline \multicolumn{4}{|c|}{$\begin{array}{l}3 \text { - optional printout (print averaged coolant temperatures in file mixture_temp.out) - may not be } \\
\text { functional }\end{array}$} \\
\hline \multicolumn{4}{|c|}{4 - optional printout (print subchannel void fraction in file void.out) } \\
\hline Integer & Condi & Groul & \\
\hline- & & & \\
\hline
\end{tabular}




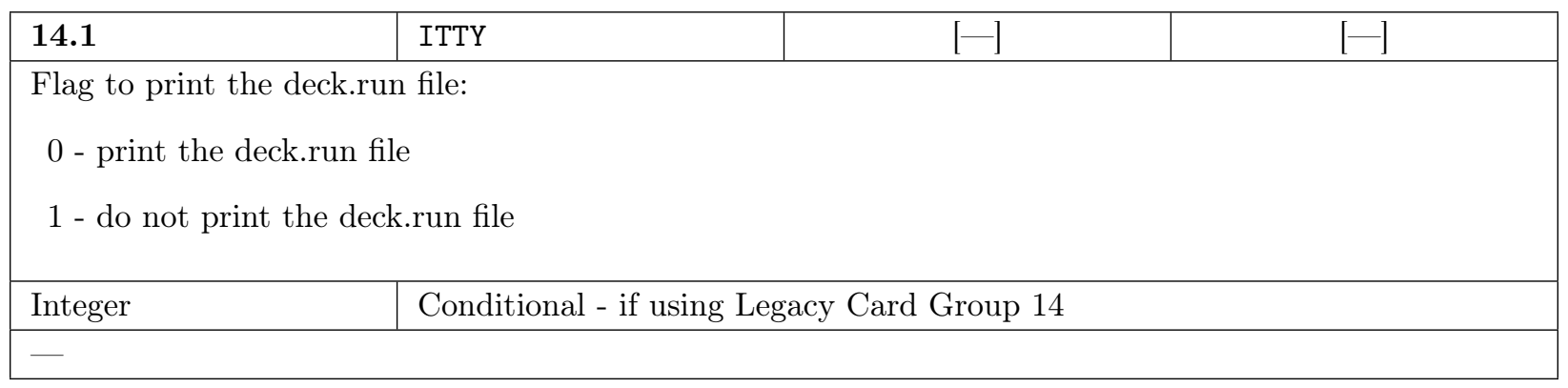

\begin{tabular}{|c|c|c|c|}
\hline 14.1 & DNB & {$[-]$} & {$[-]$} \\
\hline \multicolumn{4}{|c|}{ Flag to print the dnb.out file: } \\
\hline \multicolumn{4}{|c|}{0 - print the dnb.out file } \\
\hline \multicolumn{4}{|c|}{1 - do not print the dnb.out file } \\
\hline Integer & Cond & Grou & \\
\hline- & & & \\
\hline
\end{tabular}

\begin{tabular}{|c|c|c|c|}
\hline 14.1 & IMASS & {$[-]$} & {$[-]$} \\
\hline \multicolumn{4}{|c|}{ Flag to print the mass_balance.out file: } \\
\hline \multicolumn{4}{|c|}{0 - print the mass_balance.out file } \\
\hline \multicolumn{4}{|c|}{1 - do not print the mass_balance.out file } \\
\hline Integer & Condit & Groul & \\
\hline- & & & \\
\hline
\end{tabular}

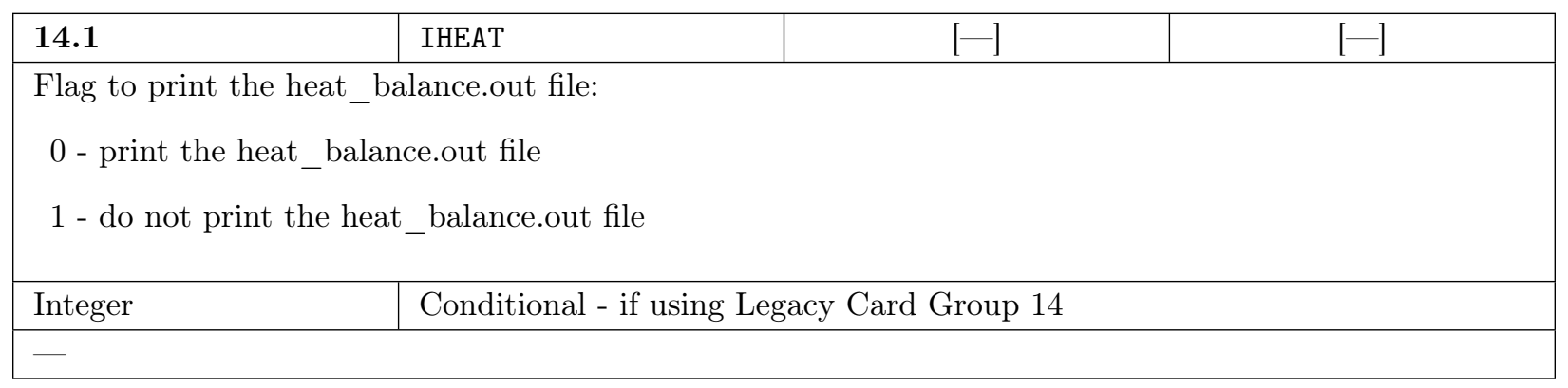




\begin{tabular}{|c|c|c|c|}
\hline 14.1 & ICHAN & {$[-]$} & {$[-]$} \\
\hline \multicolumn{4}{|c|}{ Flag to print the results_channels.out file: } \\
\hline \multicolumn{4}{|c|}{0 - print the results_channels.out file } \\
\hline \multicolumn{4}{|c|}{1 - do not print the results_channels.out file } \\
\hline Integer & Condit & Grou & \\
\hline - & & & \\
\hline
\end{tabular}

\begin{tabular}{|c|c|c|c|}
\hline 14.1 & IGAP & {$[-]$} & {$[-]$} \\
\hline \multicolumn{4}{|c|}{ Flag to print the results_gap.out file: } \\
\hline \multicolumn{4}{|c|}{0 - print the results_gap.out file } \\
\hline \multicolumn{4}{|c|}{1 - do not print the results_gap.out file } \\
\hline Integer & Condi & Grou & \\
\hline - & & & \\
\hline
\end{tabular}

\begin{tabular}{|c|c|c|c|}
\hline 14.1 & KRY & {$[-]$} & {$[-]$} \\
\hline \multicolumn{4}{|c|}{ Flag to print the krysolv.out file: } \\
\hline \multicolumn{4}{|c|}{0 - print the krysolv.out file } \\
\hline \multicolumn{4}{|c|}{1 - do not print the krysolv.out file } \\
\hline Integer & Con & Grou & \\
\hline — & & & \\
\hline
\end{tabular}

CARD 14.2 is read only if $\mathrm{N} 1 \neq 3$ and NOUT1 $>0$

CARD 14.2: $\operatorname{PRINTC(I);~I=1:NOUT1~}$

\begin{tabular}{|l|l|c|}
\hline $\mathbf{1 4 . 2}$ & PRINTC (I) & {$[-]$} \\
\hline $\begin{array}{l}\text { Index numbers of channels } \text { to be printed. Sixteen values are entered per card line. Repeat until NOUT1 } \\
\text { values have been entered. }\end{array}$ \\
\hline Integer & Conditional - if using Legacy Card Group 14 \\
\hline Must be a channel index declared in model \\
\hline
\end{tabular}

CARD 14.3 is read only if $\mathrm{N} 1=2$ or 5 and NOUT3 $>0$

CARD 14.3: PRINTG(I); I=1:NOUT3 


\begin{tabular}{|l|l|c|}
\hline $\mathbf{1 4 . 3}$ & PRINTG(I) & {$[-]$} \\
\hline $\begin{array}{l}\text { Index numbers of gaps to be printed. Sixteen values are entered per card line. Repeat until NOUT3 } \\
\text { values have been entered. }\end{array}$ \\
\hline Integer & Conditional - if using Legacy Card Group 14 \\
\hline Must be a gap index declared in model \\
\hline
\end{tabular}

CARD 14.4 is read only if $\mathrm{N} 1>2$ and NOUT2 $>0$

CARD 14.2: PRINTR(I); I=1:NOUT2

\begin{tabular}{|c|c|c|c|}
\hline 14.4 & PRINTR (I) & {$[-]$} & {$[-]$} \\
\hline \multicolumn{4}{|c|}{$\begin{array}{l}\text { Index numbers of rods to be printed. Sixteen values are entered per card line. Repeat until NOUT2 } \\
\text { values have been entered. }\end{array}$} \\
\hline Integer & Conditiona & Groul & \\
\hline
\end{tabular}

CARD 14.5 is read only if $\mathrm{N} 1>2$ and NOUT4 $>0$

CARD 14.5: PRINTHS(I); I=1:NOUT4

\begin{tabular}{|l|l|c|}
\hline $\mathbf{1 4 . 5}$ & PRINTHS (I) & {$[-]$} \\
\hline $\begin{array}{l}\text { Index numbers of unheated conductors to be printed. Sixteen values are entered per card line. Repeat } \\
\text { until NOUT2 values have been entered. }\end{array}$ \\
\hline Integer & Conditional - if using Legacy Card Group 14 \\
\hline
\end{tabular}

\subsection{New Card Group 14}

Use the new input format by entering a negative 14 instead of a positive one to start Card Group 14. Each option is simply entered as a key-value pair entered on separate lines (1 pair per line). The key-value pairs may be entered in any order. Not entering a key-value pair will result in the default behavior being used. This card must be terminated with the phrase "end 14" as the final line of this card group input.

This card group is read by subroutine READ_CARD_14_ALT.

The first line indicates the group number: NGROUP $=-14$ 


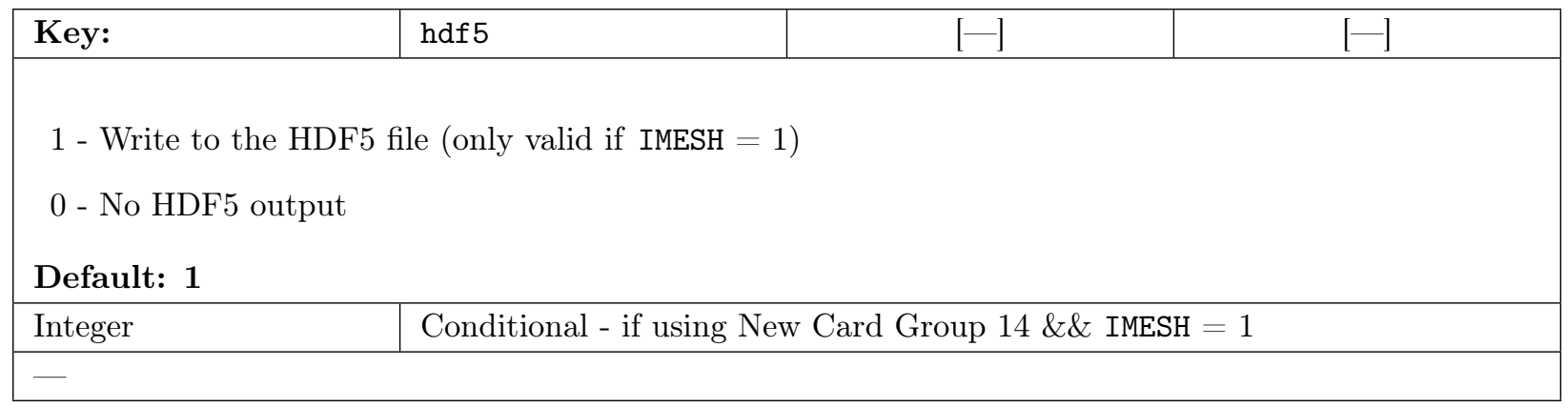

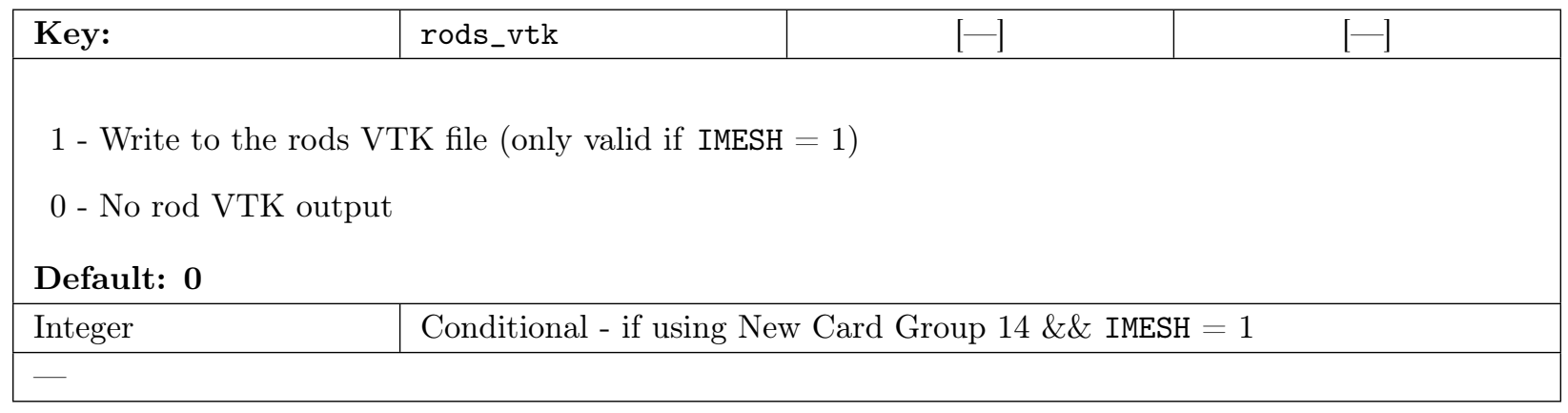

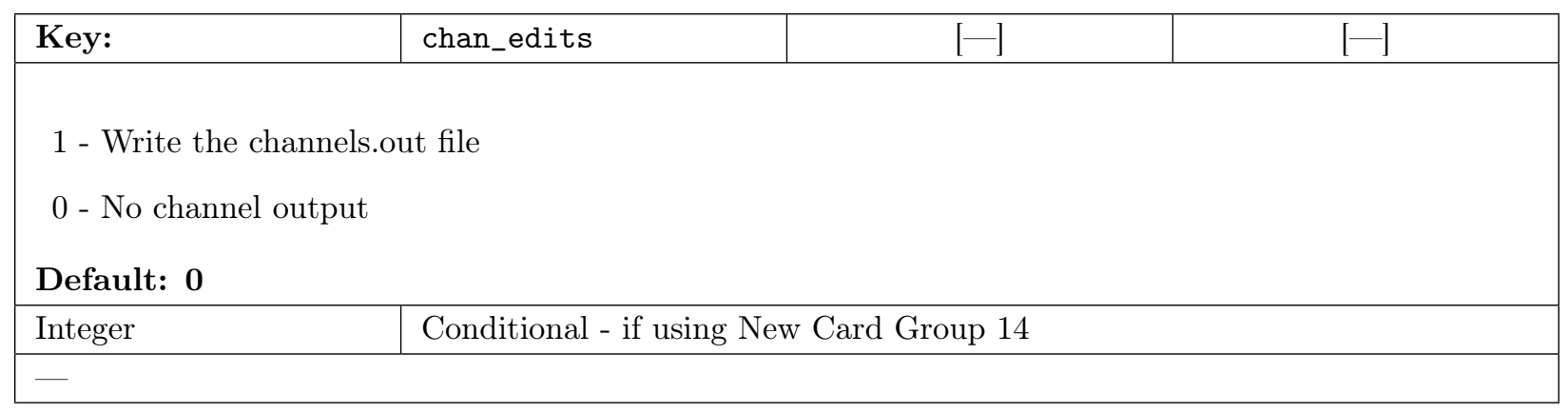

\begin{tabular}{|c|c|c|c|}
\hline Key: & rod_edits & {$[-]$} & {$[-]$} \\
\hline \multicolumn{4}{|c|}{$\begin{array}{l}1 \text { - Write the conductor data to the deck.out file } \\
0 \text { - No rod/conductor output }\end{array}$} \\
\hline \multicolumn{4}{|l|}{ Default: 0} \\
\hline Integer & Conditiona & oup 14 & \\
\hline- & & & \\
\hline
\end{tabular}




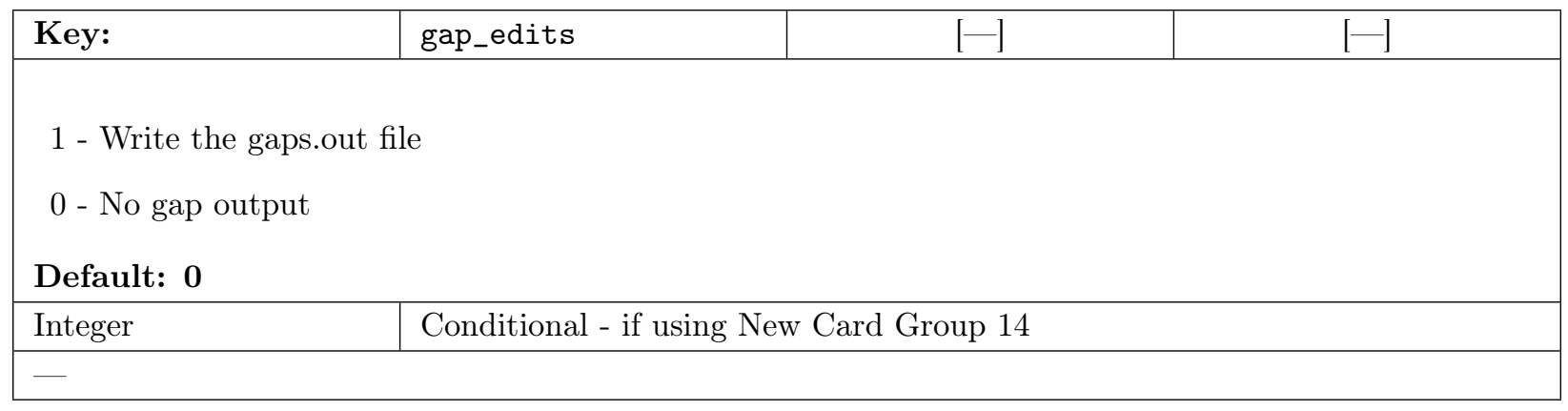

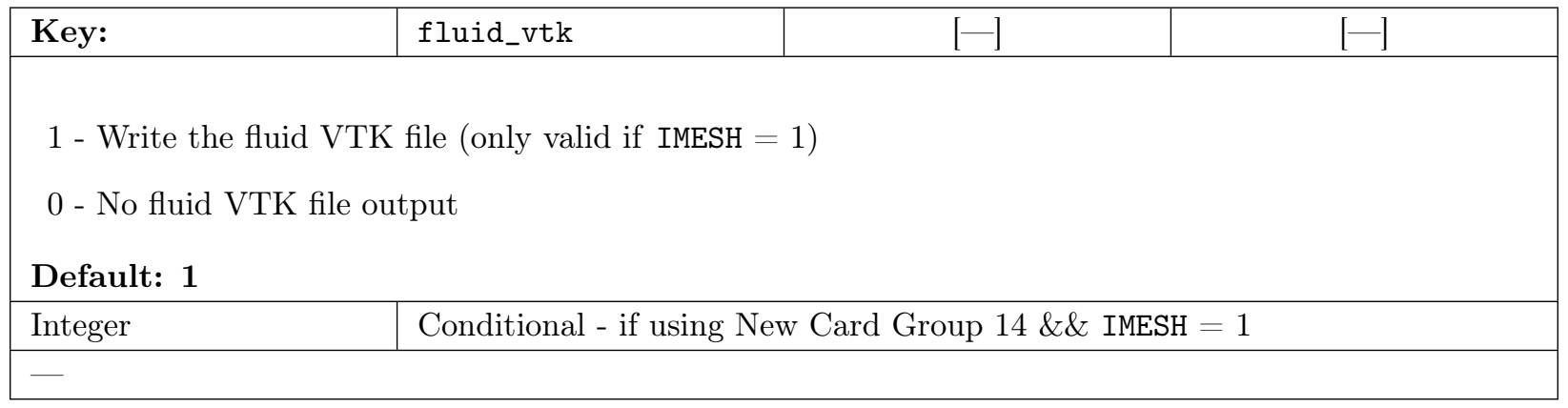

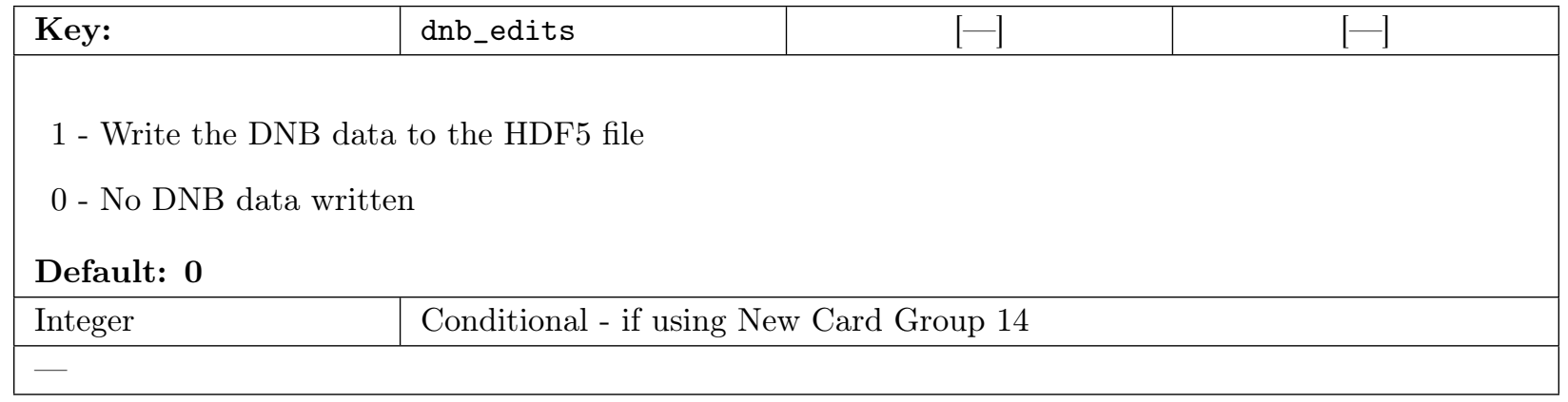

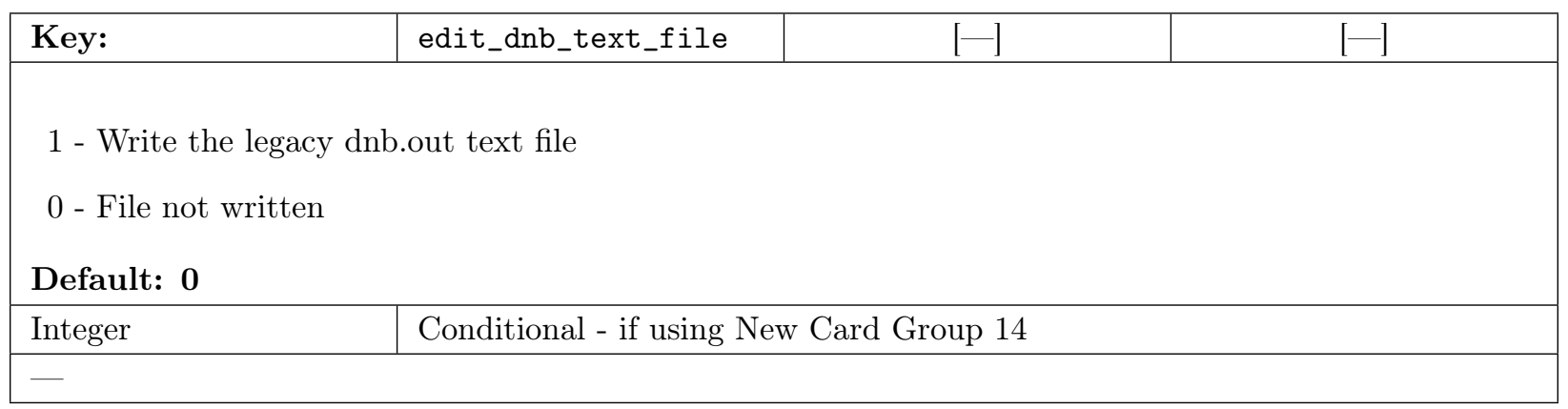




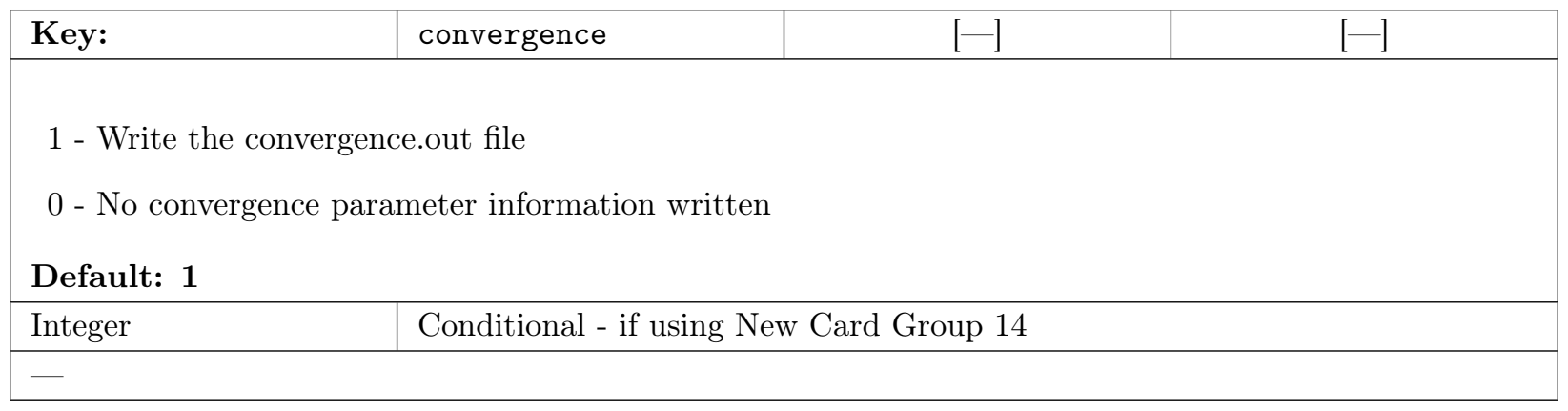

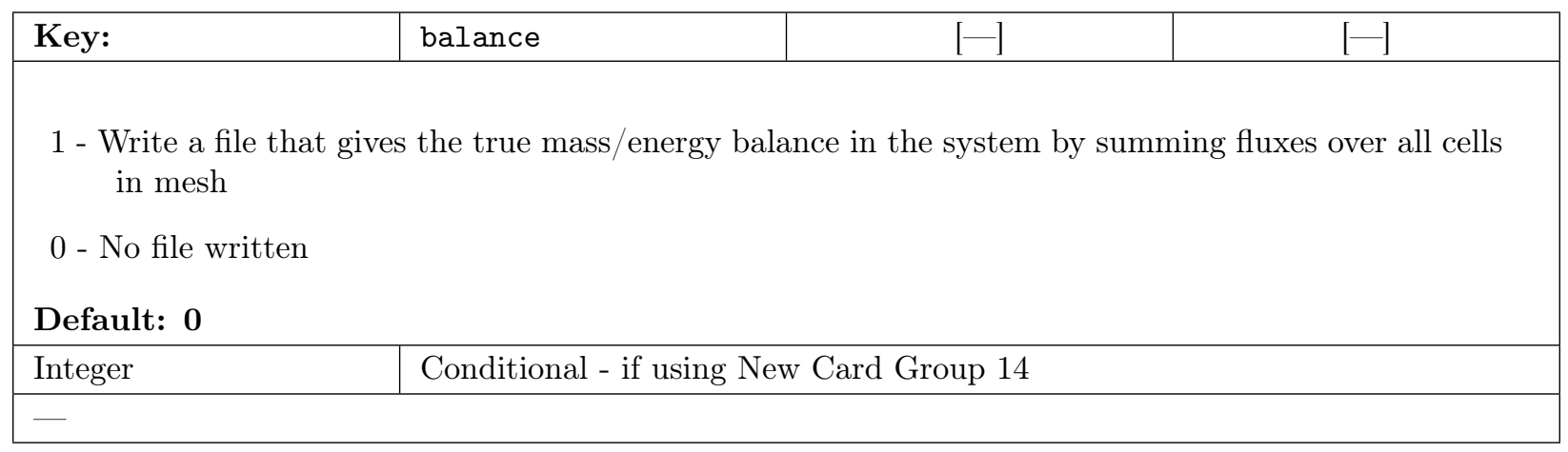

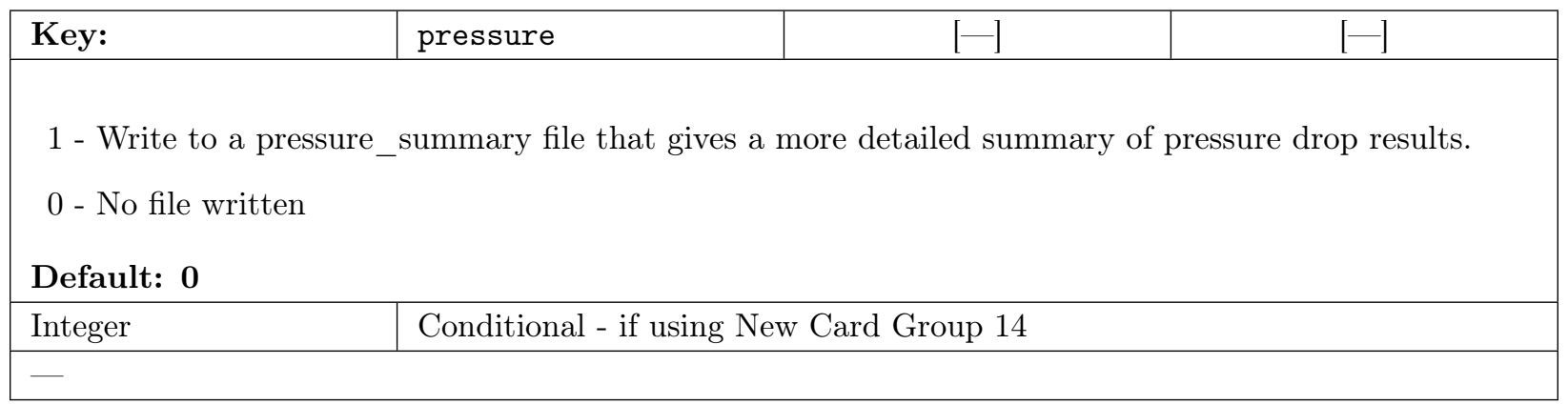

\begin{tabular}{|c|c|c|c|}
\hline Key: & native_hdf5 & {$[-]$} & {$[-]$} \\
\hline \multicolumn{4}{|c|}{$\begin{array}{l}1 \text { - Write output data to a native HDF5 file (does not use VERA-CS format and is therefore applicable } \\
\text { to arbitrary geometry types) }\end{array}$} \\
\hline \multicolumn{4}{|c|}{0 - No file written } \\
\hline \multicolumn{4}{|c|}{ Default: 0} \\
\hline Integer & Conditional - & oup 1 & \\
\hline - & & & \\
\hline
\end{tabular}




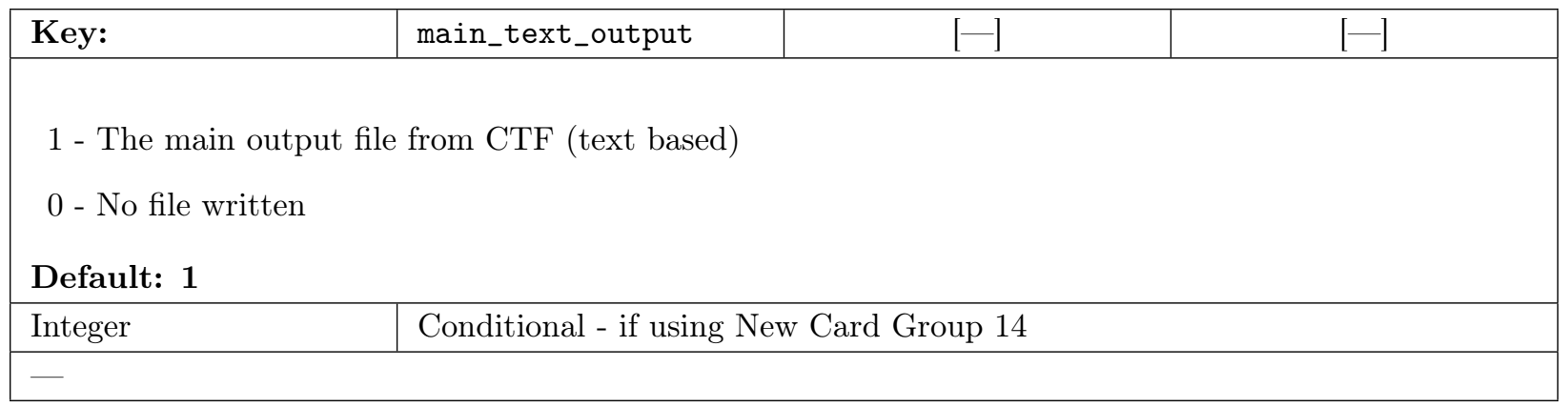

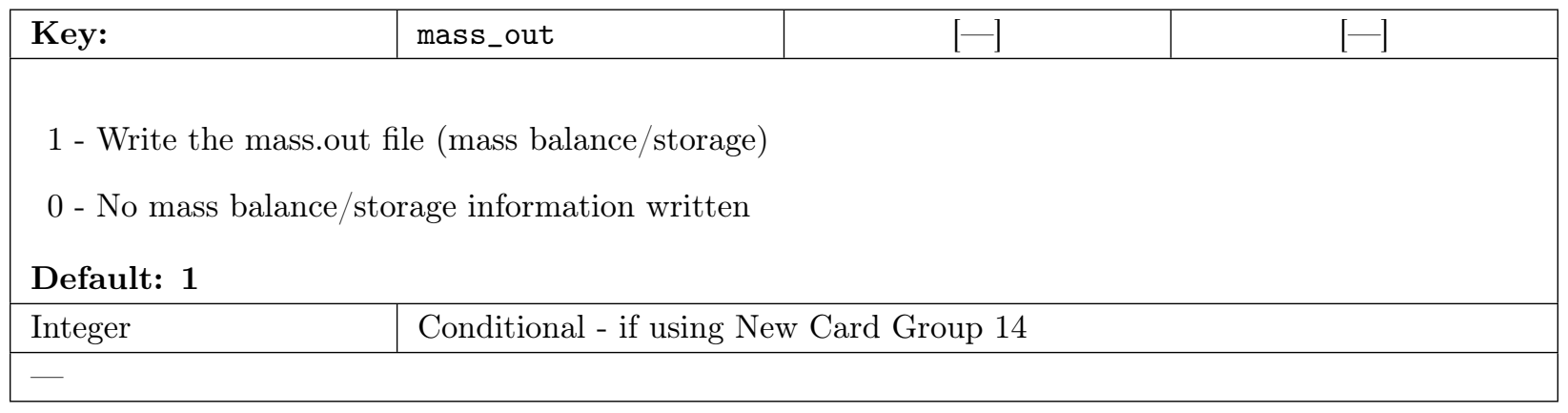

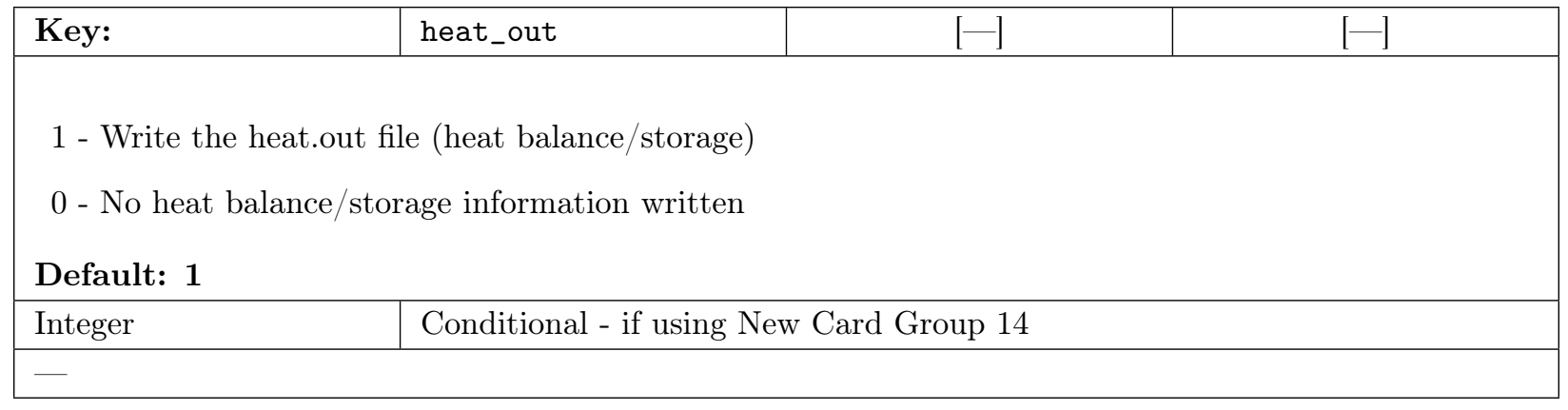

\begin{tabular}{|c|c|c|c|}
\hline Key: & run_out & {$[-]$} & {$[-]$} \\
\hline \multicolumn{4}{|c|}{$\begin{array}{l}1 \text { - Write the run.out file (timestep/iteration information) } \\
0 \text { - No timestep/iteration information written } \\
\text { Default: } 1\end{array}$} \\
\hline Integer & Condition & & \\
\hline- & & & \\
\hline
\end{tabular}




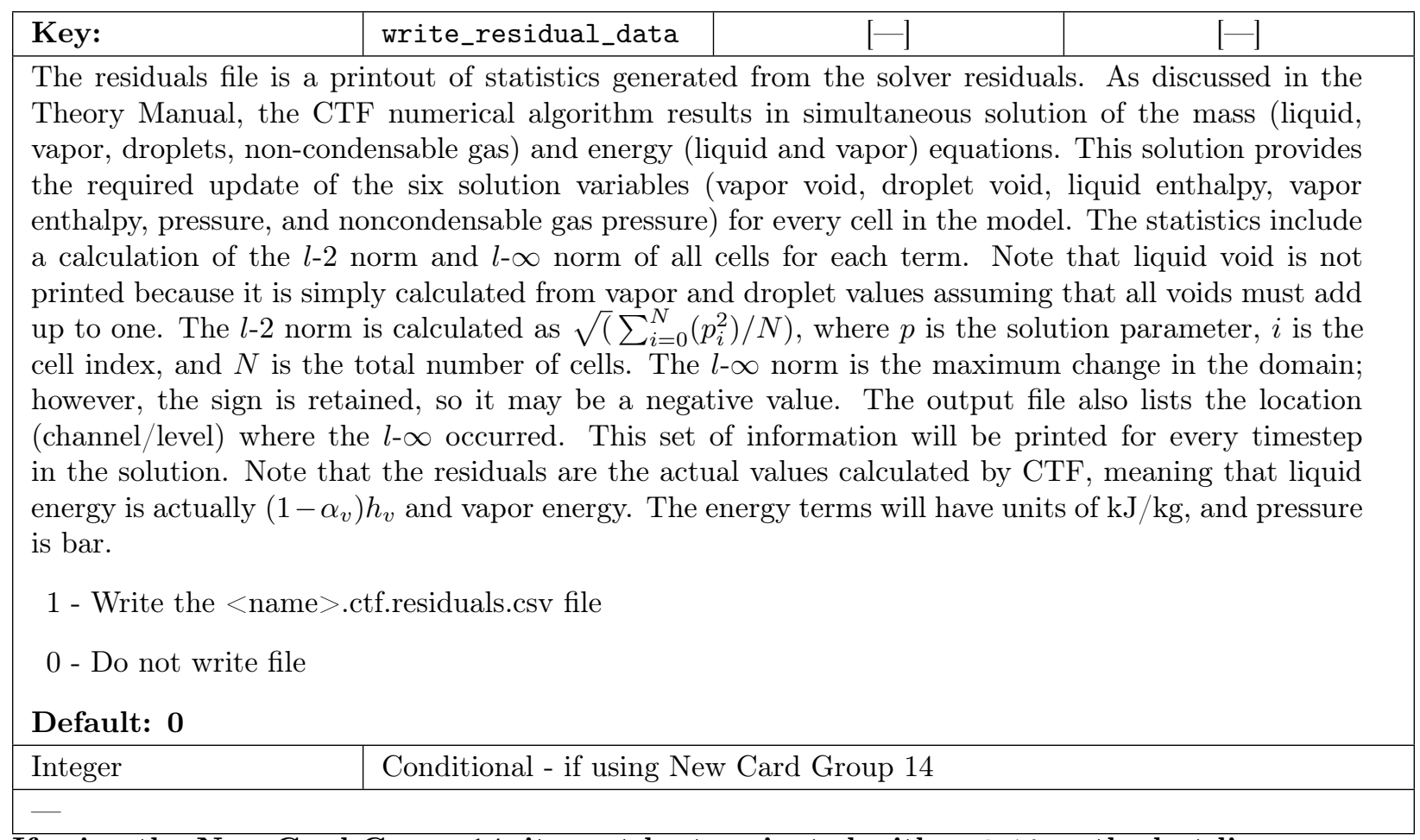

If using the New Card Group 14, it must be terminated with end 14 as the last line

Example usage of the New Card Group 14:

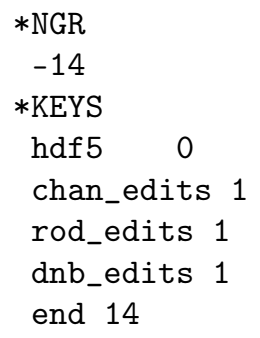




\section{Card Group 15}

This card group is read by subroutine READ_CARD_15.

After all component data have been entered, the user must define the time domain for the simulation. The total time can be divided into several domains of specified duration. Each time domain can have different minimum and maximum time step sizes and different edit intervals.

If modeling a full transient (i.e. NOTRANS $=0$ ), then any number of groups of time data may be entered, with each group specified by its own line in CARD 15.1. The last line of CARD 15.1 should give DTMIN as a negative number. This will prompt CTF to stop reading. For example, if only specifying one time data group, there should be 2 lines; the first line gives the time data for the transient and the second line terminates the read of Card Group 15.

If modeling a pseudo-transient (i.e. NOTRANS $=1$ ), then only one line of time data should be entered. The minimum and maximum timestep sizes and the RTWFP values will be used by the code, but the TEND and EDINT values will be ignored. Do not enter more than one line on CARD 15.1 if NOTRANS $=1$, as this will cause a read-error by CTF.

The first line indicates the group number: NGROUP $=15$

CARD 15.1: DTMIN, DTMAX, TEND, EDINT, DMPINT, RTWFP, MAXITS

\begin{tabular}{|c|c|c|c|}
\hline 15.1 & DTMIN & {$[\mathrm{s}]$} & {$[\mathrm{s}]$} \\
\hline \multicolumn{4}{|c|}{$\begin{array}{l}\text { Minimum time step allowed for this time domain. } \\
\text { Enter a negative value to terminate the calculation. }\end{array}$} \\
\hline Float & Required & & \\
\hline$>0.0$ & & & \\
\hline
\end{tabular}

\begin{tabular}{|l|l|l|l|}
\hline $\mathbf{1 5 . 1}$ & DTMAX & {$[\mathrm{s}]$} & {$[\mathrm{s}]$} \\
\hline \multicolumn{2}{|l|}{ Maximum time step allowed for this domain } & \\
\hline Float & Required & \\
\hline$>0.0$ & \\
\hline
\end{tabular}




\begin{tabular}{|l|l|l|l|}
\hline $\mathbf{1 5 . 1}$ & TEND & {$[\mathrm{s}]$} & {$[\mathrm{s}]$} \\
\hline $\begin{array}{l}\text { End of this time domain } \\
\text { (related to the total time from the beginning at } t=0 \mathrm{~s}\end{array}$ \\
\hline Float & Required \\
\hline$>0.0$ & \\
\hline
\end{tabular}

\begin{tabular}{|c|c|c|c|}
\hline 15.1 & EDINT & {$[\mathrm{s}]$} & {$[\mathrm{s}]$} \\
\hline \multicolumn{4}{|c|}{$\begin{array}{l}\text { Print interval for this time domain } \\
\text { Output speciified in CARD GROUP } 14 \text { will be printed every EDINT seconds (related to the beginning } \\
\text { of the current time domain) }\end{array}$} \\
\hline Float & Required & & \\
\hline$>=0.0$ & & & \\
\hline
\end{tabular}

\begin{tabular}{|c|c|c|c|}
\hline 15.1 & DMPINT & {$[-]$} & {$[-]$} \\
\hline \multicolumn{4}{|c|}{$\begin{array}{l}\text { Restart dump interval } \\
\text { Data for restart will be saved every DMPINT seconds (related to the beginning of the current time } \\
\text { domain). The file will be overwritten each time. One can start only from the time step which was } \\
\text { saved last. } \\
\text { Note: In case DUMPF =0, the input value DMPINT is not used. In case DUMPF }=1 \text { and DMPINT }=0 \text {, only } \\
\text { one restart file is written at the end of the calculation. }\end{array}$} \\
\hline Float & Required & & \\
\hline$>0.0$ & & & \\
\hline
\end{tabular}

\begin{tabular}{|c|c|c|c|}
\hline 15.1 & RTWFP & {$[-]$} & {$[-]$} \\
\hline \multicolumn{4}{|c|}{ Ratio of time step sizes for heat conduction solution and fluid solution } \\
\hline \multicolumn{4}{|c|}{$\begin{array}{l}\text { To obtain steady-state conditions, the conduction solution can generally use time steps greater than } \\
\text { the fluid solution. }\end{array}$} \\
\hline \multicolumn{4}{|c|}{ For transient calculations, RTWFP should be 1.0} \\
\hline \multicolumn{4}{|c|}{ Note: Ratios of $R T W F P<1$ are not allowed. In this case, the code automatically sets $R T W F P=1.0$} \\
\hline Float & Require & & \\
\hline
\end{tabular}

\begin{tabular}{|c|c|c|c|}
\hline 15.1 & MAXITS & {$[-]$} & {$[-]$} \\
\hline \multicolumn{4}{|c|}{$\begin{array}{l}\text { Enter only if NOTRANS }=1 \text { (for modeling a pseudo-transient). This gives the maximum number of } \\
\text { iterations that should be run before ending the simulation and printing results. }\end{array}$} \\
\hline Integer & Conditi & & \\
\hline \multicolumn{4}{|l|}{$>=1.0$} \\
\hline
\end{tabular}




\section{Card Group 16}

This card group is currently not functional. It was put here to read in meshing data. Currently, meshing data are entered on Card Groups 2 and 3. It is intended that eventually the meshing information will be migrated from those card groups to this one for better organization of the input deck. Furthermore, the mesh is setup in CTF in a somewhat inefficient way right now: the cells are all free-standing, not knowing how they connect to one another. This leads to larger-than-necessary VTK files being produced. Eventually, this will be remedied and the necessary information for generating the new meshes will be put in this Card Group. Backwards compatibility will be retained for decks created using the old meshing technique using the IMESH option on Card Group 1. 


\section{Card Group 17}

This group gives a top-view of the map of channels and rods in the model. It is necessary for writing HDF5 edits. If the MAPS variable on Card 1.1 is set to 1, this Card Group must be entered. If MAPS was set to 0, do not enter this group. CTF only supports square rod lattice geometry. Special consideration must be given to which assemblies own which rods and channels. Special considerations are also required for symmetry cases. It is not intended that this card group should be generated by hand. Rather, the PWR preprocessor should be used to create the input deck when HDF5 output is desired.

This card group is read by subroutine READ_CARD_17.

The first line indicates the group number: NGROUP $=17$

CARD 17.1: HDF5 NAME, VTK NAME

\begin{tabular}{|l|l|c|c|}
\hline $\mathbf{1 7 . 1}$ & HDF5 NAME & {$[-]$} & {$[-]$} \\
\hline Has no use, but required & & \\
\hline Character & Conditional - if MAPS $\neq 0$ \\
\hline Any string
\end{tabular}

\begin{tabular}{|l|l|c|c|}
\hline $\mathbf{1 7 . 1}$ & VTK NAME & {$[-]$} & {$[-]$} \\
\hline Has no use, but required & & \\
\hline Character & Conditional - if MAPS $\neq 0$ \\
\hline Any string
\end{tabular}

CARD 17.2: TOTRODSROW, TOTRODSCOL

\begin{tabular}{|l|l|l|}
\hline $\mathbf{1 7 . 2}$ & TOTRODSROW & {$[-]$} \\
\hline $\begin{array}{l}\text { The number of rods in a single row of rods in the entire core model } \\
\text { See Figure 19.1 for reference. This particular model would have 9 rods in a row. }\end{array}$ \\
\hline Integer & Conditional - if MAPS $\neq 0$ \\
\hline$>=1$ &
\end{tabular}




\begin{tabular}{|l|l|l|}
\hline $\mathbf{1 7 . 2}$ & TOTRODSCOL & {$[-]$} \\
\hline $\begin{array}{l}\text { The number of rods in a single column of rods in the core model } \\
\text { Figure 19.1 would have 9 rods in a column. }\end{array}$ \\
\hline Integer & Conditional - if MAPS $\neq 0$ \\
\hline$>=1$ & \\
\hline
\end{tabular}

CARD 17.3: TOTCHANSROW, TOTCHANSCOL

\begin{tabular}{|l|l|l|}
\hline $\mathbf{1 7 . 3}$ & TOTCHANSROW & {$[-]$} \\
\hline $\begin{array}{l}\text { The number of channels in a single row of channels in the entire core model } \\
\text { The model in Illustration 1 would have 10 channels per row. }\end{array}$ \\
\hline Integer & Conditional - if MAPS $\neq 0$ \\
\hline$>=0$ &
\end{tabular}

\begin{tabular}{|c|c|c|c|}
\hline 17.3 & TOTCHANSCOL & {$[-]$} & {$[-]$} \\
\hline \multicolumn{4}{|c|}{ The number of channels in a single column of channels in the core model } \\
\hline Integer & Conditional - & & \\
\hline \multicolumn{4}{|l|}{$>=0$} \\
\hline
\end{tabular}

\section{CARD 17.4: Rod Map}

The rod map is entered as zeros, positive integers, and negative integers. A positive integer represents the assembly index that owns a solved rod. A negative integer represents the assembly index that owns an unsolved rod, due to the rod existing on the unsolved side of a symmetry line. A "0" represents a blank region. The map must be square. When CTF reads these values in, it will assign each entity a unique ID. It will read from left to right, starting at the top row and working down. IDs are assigned in that order. The rod map for Figure 19.1 would be as shown in Table 3.

Table 3: Example Rod Map

$\begin{array}{lllllllll}0 & 0 & 0 & 1 & 1 & 1 & 0 & 0 & 0 \\ 0 & 0 & 0 & 1 & 1 & 1 & 0 & 0 & 0 \\ 0 & 0 & 0 & 1 & 1 & 1 & 0 & 0 & 0 \\ 2 & 2 & 2 & 3 & 3 & 3 & 4 & 4 & 4 \\ 2 & 2 & 2 & 3 & 3 & 3 & 4 & 4 & 4 \\ 2 & 2 & 2 & 3 & 3 & 3 & 4 & 4 & 4 \\ 0 & 0 & 0 & 5 & 5 & 5 & 0 & 0 & 0 \\ 0 & 0 & 0 & 5 & 5 & 5 & 0 & 0 & 0 \\ 0 & 0 & 0 & 5 & 5 & 5 & 0 & 0 & 0\end{array}$

If modeling this case using quarter symmetry, the assemblies that exist on the symmetry line must have both their solved and unsolved rods given in the map. The unsolved rods are shown using a negative number. A 
quarter symmetry map of this case would look as shown in Table 4.

Table 4: Example Quarter Rod Map

$\begin{array}{cccccc}-1 & -1 & -1 & -2 & -2 & -2 \\ -1 & -1 & -1 & -2 & -2 & -2 \\ -1 & 1 & 1 & 2 & 2 & 2 \\ -3 & 3 & 3 & 0 & 0 & 0 \\ -3 & 3 & 3 & 0 & 0 & 0 \\ -3 & 3 & 3 & 0 & 0 & 0\end{array}$

Note that rod surface data will also be printed to the HDF5 file. CTF does not consider orientation of the rod surfaces; only how they connect to adjacent channels. However, the PWR preprocessor has a specific way of setting up the connections that allows users to track the orientation of each rod surface. For square rod lattice geometry, there will be four quadrants of each rod (i.e. northeast, southeast, southwest, and northwest). To ensure the orientation is correctly printed to the HDF5 file, the PWR preprocessor must be utilized to generate the CTF input deck.

\section{CARD 17.5: Channel Map}

The channel map is similarly given as positive and negative integers for solved and unsolved channels. Zeros represent blank regions in the map. Its indices are assigned in the same way as the rod map. 


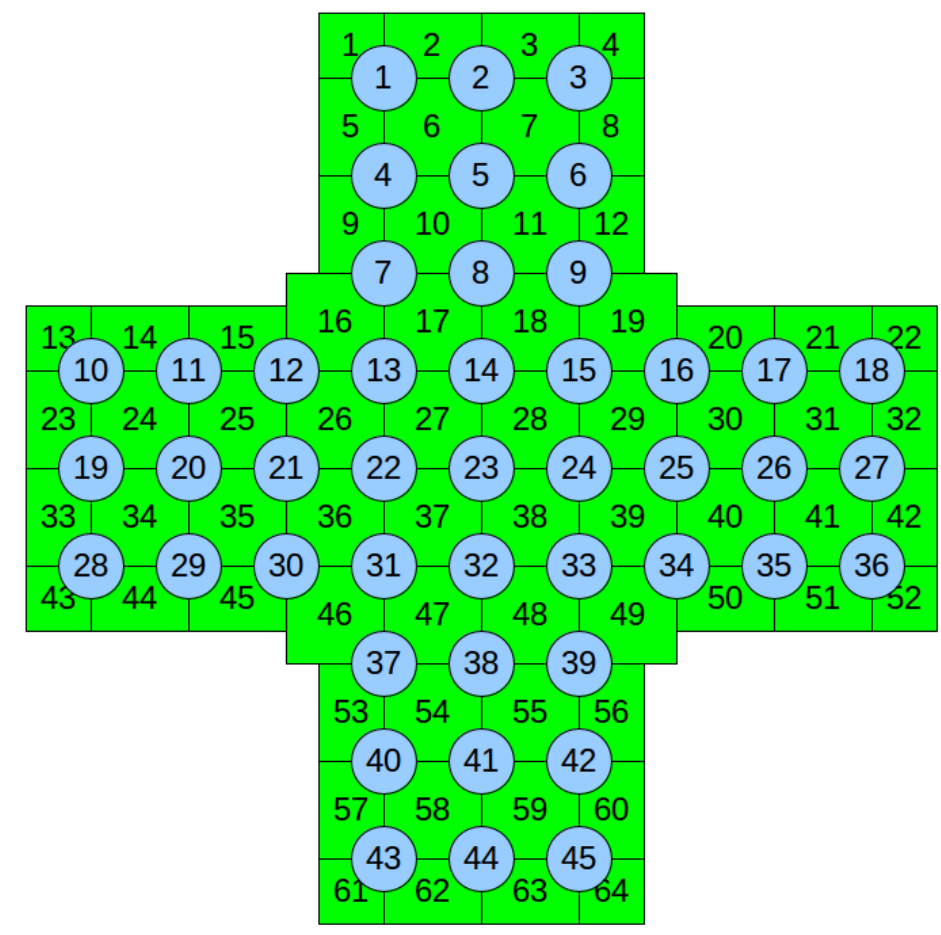

Figure 19.1: Core map of $3 \times 3$ assemblies

CARD 17.6 - Assembly Map Information: SYM_OPT, NASSEM_ROWS, NASSEM_COLS

This card is optional, but required when modeling core symmetry. One must enter the following tag prior to the card so CTF reads the input correctly:

\{assem map\}

\begin{tabular}{|c|c|c|c|}
\hline 17.6 & SYM_OPT & {$[-]$} & {$[-]$} \\
\hline \multicolumn{4}{|c|}{ The symmetry option being used: } \\
\hline \multicolumn{4}{|c|}{1 - full symmetry } \\
\hline \multicolumn{4}{|c|}{4 - quarter mirror symmetry } \\
\hline \multicolumn{4}{|c|}{5 - quarter rotational symmetry } \\
\hline Integer & Conditior & $\mathrm{m} \mathrm{me}$ & \\
\hline- & & & \\
\hline
\end{tabular}




\begin{tabular}{|l|l|c|c|}
\hline $\mathbf{1 7 . 6}$ & NASSEM_ROWS & {$[-]$} & {$[-]$} \\
\hline Number of assemblies in each row of assemblies & \\
\hline Integer & Conditional - if MAPS $\neq 0$ and $\{$ assem map $\}$ is entered \\
\hline$>=1$ & \\
\hline
\end{tabular}

\begin{tabular}{|l|l|c|c|}
\hline $\mathbf{1 7 . 6}$ & NASSEM_COLS & {$[-]$} & {$[-]$} \\
\hline Number of assemblies in each column of assemblies & \\
\hline Integer & Conditional - if MAPS $\neq 0$ and $\{$ assem map $\}$ is entered \\
\hline$>=1$ & \\
\hline
\end{tabular}

CARD 17.7 - Assembly Map

If CARD 17.6 is entered, then CARD 17.7 must be entered as well. This is a top view map of the core with each index representing an assembly in the core. Always enter the full symmetry map. For Figure 19.1, the assembly would look as shown in Table 5.

Table 5: Example full symmetry core map

$\begin{array}{lll}0 & 1 & 0 \\ 2 & 3 & 4 \\ 0 & 5 & 0\end{array}$

When modeled using quarter symmetry, this map would look as shown in Table 6:

Table 6: Example quarter rotational core map

$$
\begin{array}{lll}
0 & 3 & 0 \\
2 & 1 & 2 \\
0 & 3 & 0
\end{array}
$$

A quarter rotational symmetry map must have unique assembly indices on the symmetry lines, so it is not a true rotational symmetry. An example is shown in Table 7.

Table 7: Example quarter rotational core map

$\begin{array}{lllll}0 & 8 & 7 & 6 & 0 \\ 6 & 5 & 4 & 5 & 8 \\ 3 & 2 & 1 & 2 & 3 \\ 8 & 5 & 4 & 5 & 6 \\ 0 & 6 & 7 & 8 & 0\end{array}$

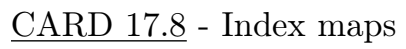


This input specified the unique index of every rod and channel in the model. The index information is added as 2D top-view maps, just as was done with the rod and channel maps. This input is optional, and it allows the user to specify an indexing scheme that is different than the default scheme used by CTF, which is left-to-right, top-to-bottom. In other words, if this is not entered, then the top-left rod will be Rod 1, and the top-right rod will be $\operatorname{Rod} N$, where $N$ is the number of rods in the first row. Rod $N+1$ will be the left-most rod of the second row, and so on. If this input is going to be used, then the tag \{index maps\} must first be entered on its own line. After that, the rod index map is entered in its entirety, and then the channel index map is entered in its entirety.

If modeling a quarter symmetry core, then only the solved assemblies shall be entered. If an assembly is split by the symmetry line, then all entities in that assembly must appear in the map, with the unsolved entities being set to a value of zero. Any blank spots in the map shall be given as zero. All indices in the map shall be unique.

An example of a core that has 5 assemblies in a cross configuration, modeled at quarter symmetry, and containing assemblies with $3 \times 3$ rods, could have the following rod index map shown in Table 8 .

Table 8: Example quarter symmetry rod index map

$\begin{array}{cccccc}0 & 0 & 0 & 0 & 0 & 0 \\ 0 & 1 & 2 & 5 & 6 & 7 \\ 0 & 3 & 4 & 8 & 9 & 10 \\ 0 & 11 & 12 & 0 & 0 & 0 \\ 0 & 13 & 14 & 0 & 0 & 0 \\ 0 & 15 & 16 & 0 & 0 & 0\end{array}$

\section{CARD 17.8 - Inlet orifice map}

This input is intended to be used for BWR models only (BWRMODEL set to 1 on Card 1.1). This feature allows the specification of an inlet form loss and flow area for each assembly location in the core. When CTF performs the pressure balance iteration loop to balance core pressure drop by redistributing inlet mass flow rate, it will use these values to determine the additional pressure losses associated with inlet orificing. To use this feature, two inputs are needed: the inlet form loss coefficient for each assembly, and the inlet flow area of each orifice leading into each assembly. Each of these lists must be sized to be equal to the number of assemblies in the full core model. Even when modeling symmetry, one value must be given for each assembly in the full core. The values are specified starting with the top-left assembly, moving from left-to-right, and then the second row is specified in this same manner, and so on. The inlet form losses are specified by first entering the tag \{inlet_orifice_map\} on its own line. The inlet flow areas are specified by first entering the tag \{inlet_orifice_area\} on its own line. The following shows an example input for a model of 5 assemblies in a cross configuration.

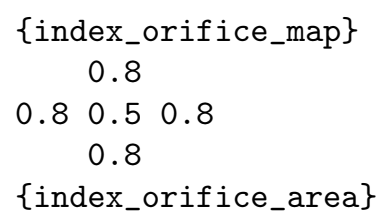




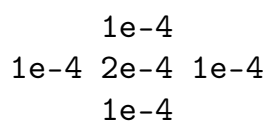

If this input is being entered, then the assembly map (Card 17.6) must also have been entered previously. 


\section{Card Group 18}

This group specifies a set of convergence criteria that will be used to judge when the transient has reached steady state. This only has an effect if the user specified NOTRANS $=1$ in Card 1.1. If this card is not specified, a default set of convergence criteria will be used. This card group specifies an older set of convergence criteria that use mass and energy balance and storage as the criteria. The meaning of these terms are not as intuitive as watching actual solution terms become steady. A new set of convergence criteria that are based on the $l_{\infty}$ of several solution variables are provided in Card Group 19 and they are recommended over these criteria. Enter Card Group 18 to use the older criteria or enter Card Group 19 to use the newer criteria. Do not enter both card groups. A basic description of the terms may be found here. Consult the CTF Theory Manual [1] for a more in-depth description of these terms.

The first line indicates the group number: NGROUP $=18$

CARD 18.1: Global energy balance

\begin{tabular}{|l|l|l|l|}
\hline $\mathbf{1 8 . 1}$ & ENERGYBAL & {$[\%]$} & {$[\%]$} \\
\hline Energy in minus energy out, normalized to energy in. & \\
\hline Float & Required & \\
\hline$>0.0$ &
\end{tabular}

\begin{tabular}{|l|l|c|c|}
\hline $\mathbf{1 8 . 2}$ & MASSBAL & {$[\%]$} & {$[\%]$} \\
\hline \multicolumn{2}{|l|}{ Mass in minus mass out, normalized to mass in. } \\
\hline Float & Required & \\
\hline$>0.0$ &
\end{tabular}

\begin{tabular}{|l|l|l|l|}
\hline $\mathbf{1 8 . 3}$ & FESTOR & {$[\%]$} & {$[\%]$} \\
\hline \multicolumn{2}{|l|}{ Energy stored in the fluid, normalized by system power } \\
\hline Float & Required \\
\hline$>0.0$
\end{tabular}




\begin{tabular}{|l|l|l|l|}
\hline $\mathbf{1 8 . 4}$ & SESTOR & {$[\%]$} & {$[\%]$} \\
\hline \multicolumn{2}{|l|}{ Energy stored in the solid, normalized by system power } \\
\hline Float & Required & \\
\hline$>0.0$ & \\
\hline
\end{tabular}

\begin{tabular}{|l|l|l|l|}
\hline $\mathbf{1 8 . 5}$ & MSTOR & {$[\%]$} & {$[\%]$} \\
\hline \multicolumn{2}{|c|}{ mass stored in the coolant, normalized by inlet mass flow } & \\
\hline Float & Required & \\
\hline$>0.0$ & \\
\hline
\end{tabular}




\section{Card Group 19}

This group specifies a set of stopping criteria that will be used to judge when the transient has reached steady state. This only has an effect if the user specified NOTRANS=1 in Card 1.1. This set of stopping criteria is an alternative to the set that may be specified on Card 18. If neither Card 18 or 19 are specified, the code will default to using the stopping criteria of Card 18 (default values will be used). This card group specifies a newer set of stopping criteria that use the normalized $l_{\infty}$-norm and $l_{2}$-norm of the difference between solution vectors at set intervals of time in the simulation. The $l_{\infty}$ and $l_{2}$ is checked for:

1. Void

2. Pressure

3. Coolant temperature

4. Solid temperature

5. Liquid axial velocity

6. Vapor axial velocity

7. Droplet axial velocity

Each parameter (except void) is checked against both a relative and an absolute tolerance. Void is dimensionless, so it is only checked against an absolute tolerance. A steady-state run of the code will converge if the following conditions holds true.

$$
\begin{gathered}
{\left[\frac{\max \left(\left|x^{n}-x^{n-1}\right|\right)}{x^{n-1}}<=R_{\infty}, \text { or } \max \left(\left|x^{n}-x^{n-1}\right|\right)<=A_{\infty}\right], \text { and }} \\
{\left[\sqrt{\frac{\sum_{k=1}^{N}\left(\frac{x^{n}-x^{n-1}}{x^{n-1}}\right)^{2}}{N}}<=R_{2}, \text { or } \sqrt{\frac{\sum_{k=1}^{N}\left(x^{n}-x^{n-1}\right)^{2}}{N}}<=A_{2}\right]}
\end{gathered}
$$

In this equation, $x$ is a vector of the solution for all mesh cells in the model (i.e., pressure, coolant temperature, etc.). The exponent, $n$, represents the point in time where a check has been made. Solution vectors are 
compared at $0.05 \mathrm{~s}$ intervals in the transient solution. The first two equations are comparing the relative $l_{\infty}$ and absolute $l_{\infty}$ against relative, $R$, and absolute, $A$, stopping criteria. Note that $A$ has units and $R$ is dimensionless. The second two equations compare the relative $l_{2}$ and absolute $l_{2}$ against its separate relative and absolute stopping criteria. As the equations indicate, the $l_{\infty}$ must meet either the relative or absolute criteria and the $l_{2}$ must meet either its relative or absolute criteria. Both the $l_{2}$ and $l_{\infty}$ must satisfy at least one of its criteria for the simulation to be completed.

There are two caveats: first, the denominators have a floor value of $1 \cdot 10^{-16}$ to prevent divide-by-zero errors and, second, the code will take one more iteration (one more timestep) and double-check that the solution is still below the tolerance. As long as the code still meets the stopping criteria, the code will exit.

The benefit of the relative stopping criteria is that it defines the number of digits to which each solution term is steady. For example, if CTF satisfies a relative stopping criteria of 1E-5 for solid temperatures, and a given temperature in the solution is 300.01, the user knows that those five digits will not change any further. The solution can oscillate in the sixth digit, but this small amount of wiggle in the solution may be acceptable for the user's application. The absolute tolerance is added as a safety measure to protect against situations where some solved quantity becomes very small. As seen in Equation 21.1, the relative stopping criteria is normalized by the old time solution value, $x^{n}$. If some velocity, for example, is close to zero, the relative change in the term can be huge from one checkpoint to another, even though the absolute change may be very small and the solution essentially steady.

The void difference does not check against a relative tolerance because, frequently, the void will be very small, leading to very large relative differences from one check to another; it is only checked against the absolute tolerance. In addition to Equation 21.1, CTF will also check the mass and energy balance of the solution domain. Normalized mass balance is defined in Equation 21.2 and normalized energy balance is defined in Equation 21.3.

$$
\begin{gathered}
M_{\text {balance }}=\frac{M_{\text {inlet }}-M_{\text {outlet }}}{M_{\text {inlet }}} \cdot 100 \\
E_{\text {balance }}=\frac{Q_{\text {rod }}+Q_{\text {fluid }}+Q_{\text {inlet }}-Q_{\text {outlet }}-Q_{\text {amb }}}{Q_{\text {rod }}+Q_{\text {fluid }}+Q_{\text {inlet }}} \cdot 100
\end{gathered}
$$

The terms, $M_{\text {inlet }}$ and $M_{\text {outlet }}$ define the total mass entering the system and the total mass leaving the system, respectively. In the energy equation, the terms, $Q_{\text {rod }}, Q_{\text {fluid }}, Q_{\text {inlet }}, Q_{\text {outlet }}$, and $Q_{\text {amb }}$ represent the energy generated in the solid objects, energy generated in the fluid (gamma heating), energy entering the system due to advection, energy leaving the system due to advection, and energy loss to the environment, respectively. In a steady-state system, both of these terms should be driven to zero within some tolerance.

The following three cards specify the values of the tolerances for the steady-state check. Card 19.1 specifies the relative tolerances for $l_{\infty}$, Card 19.2 specifies the absolute tolerances for $l_{\infty}$, Card 19.3 specifies the tolerances for the mass and energy balances, Card 19.4 specifies the relative tolerances for $l_{2}$, and Card 19.5 specifies the absolute tolerances for $l_{2}$. If the user wishes to use only one type of stopping criteria (i.e., either $l_{\infty}$ or $l_{2}$ ), the other set may be disabled by setting it to an arbitrarily high value.

The first line of the card group indicates the group number: NGROUP $=19$

CARD 19.1: LIPRESS, LITCOOL, LITSOLID, LIVL, LIVV, LIVD 


\begin{tabular}{|l|l|l|l|}
\hline $\mathbf{1 9 . 1}$ & LIPRESS & {$[-]$} & {$[-]$} \\
\hline Relative tolerance for the $l_{\infty}$ of pressure. Default 1E-5. \\
\hline Float & Required \\
\hline$>0.0$ & \\
\hline
\end{tabular}

\begin{tabular}{|l|l|c|c|}
\hline $\mathbf{1 9 . 1}$ & LITCOOL & {$[-]$} & {$[-]$} \\
\hline Relative tolerance for the $l_{\infty}$ of coolant temperature. Default 1E-5. \\
\hline Float & Required \\
\hline$>0.0$ & \\
\hline
\end{tabular}

\begin{tabular}{|l|l|l|l|}
\hline $\mathbf{1 9 . 1}$ & LITSOLID & {$[-]$} & {$[-]$} \\
\hline Relative tolerance for the $l_{\infty}$ of solid temperature. Default 1E-5. & \\
\hline Float & Required \\
\hline$>0.0$ & \\
\hline
\end{tabular}

\begin{tabular}{|l|l|c|c|}
\hline $\mathbf{1 9 . 1}$ & LIVL & {$[-]$} & {$[-]$} \\
\hline Relative tolerance for the $l_{\infty}$ of axial liquid velocity. Default 1E-5. & \\
\hline Float & Required \\
\hline$>0.0$ & \\
\hline
\end{tabular}

\begin{tabular}{|l|l|c|c|}
\hline $\mathbf{1 9 . 1}$ & LIVV & {$[-]$} & {$[-]$} \\
\hline \multicolumn{2}{|l|}{ Relative tolerance for the $l_{\infty}$ of axial vapor velocity. Default 1E-5. } \\
\hline Float & Required \\
\hline$>0.0$ & \\
\hline
\end{tabular}

\begin{tabular}{|c|c|c|c|}
\hline 19.1 & LIVD & {$[-]$} & {$[-]$} \\
\hline \multicolumn{4}{|c|}{ Relative tolerance for the $l_{\infty}$ of axial droplet velocity. Default $1 \mathrm{E}-5$. } \\
\hline Float & Requi & & \\
\hline$>0.0$ & & & \\
\hline
\end{tabular}

CARD 19.2: LIVOID, LIAPRESS, LIATCOOL, LIATSOLID, LIAVL, LIAVV, LIAVD

\begin{tabular}{|l|l|c|c|}
\hline $\mathbf{1 9 . 2}$ & LIVOID & {$[-]$} & {$[-]$} \\
\hline Criteria for the $l_{\infty}$ of void. Default 1E-5. & \\
\hline Float & Required & \\
\hline$>0.0$ & \\
\hline
\end{tabular}




\begin{tabular}{|l|l|c|c|}
\hline $\mathbf{1 9 . 2}$ & LIAPRESS & {$[\mathrm{bar}]$} & {$[\mathrm{psi}]$} \\
\hline Absolute tolerance for the $l_{\infty}$ of pressure. Default $0.001 \mathrm{psi}$. & \\
\hline Float & Required \\
\hline$>0.0$ &
\end{tabular}

\begin{tabular}{|c|c|c|c|}
\hline 19.2 & LIATCOOL & {$[\mathrm{C}]$} & {$[\mathrm{F}]$} \\
\hline \multicolumn{4}{|c|}{ Absolute tolerance for the $l_{\infty}$ of coolant temperature. Default $0.001 \mathrm{~F}$. } \\
\hline Float & Required & & \\
\hline$>0.0$ & & & \\
\hline
\end{tabular}

\begin{tabular}{|c|c|c|c|}
\hline 19.2 & LIATSOLID & {$[\mathrm{C}]$} & {$[\mathrm{F}]$} \\
\hline \multicolumn{4}{|c|}{ Absolute tolerance for the $l_{\infty}$ of solid temperature. Default $0.001 \mathrm{~F}$. } \\
\hline Float & Required & & \\
\hline$>0.0$ & & & \\
\hline
\end{tabular}

\begin{tabular}{|c|c|c|c|}
\hline 19.2 & LIAVL & {$[\mathrm{m} / \mathrm{s}]$} & {$[\mathrm{ft} / \mathrm{s}]$} \\
\hline \multicolumn{4}{|c|}{ Absolute tolerance for the $l_{\infty}$ of axial liquid velocity. Default $0.001 \mathrm{ft} \mathrm{s}^{-1}$. } \\
\hline Float & Require & & \\
\hline \multicolumn{4}{|l|}{$>0.0$} \\
\hline
\end{tabular}

\begin{tabular}{|c|c|c|c|}
\hline 19.2 & LIAVV & {$[\mathrm{m} / \mathrm{s}]$} & {$[\mathrm{ft} / \mathrm{s}]$} \\
\hline \multicolumn{4}{|c|}{ Absolute tolerance for the $l_{\infty}$ of axial vapor velocity. Default $0.001 \mathrm{ft} \mathrm{s}^{-1}$. } \\
\hline Float & Require & & \\
\hline
\end{tabular}

\begin{tabular}{|c|c|c|c|}
\hline 19.2 & LIAVD & {$[\mathrm{m} / \mathrm{s}]$} & {$[\mathrm{ft} / \mathrm{s}]$} \\
\hline \multicolumn{4}{|c|}{ Absolute tolerance for the $l_{\infty}$ of axial droplet velocity. Default $0.001 \mathrm{ft} \mathrm{s}^{-1}$. } \\
\hline Float & Require & & \\
\hline$>0.0$ & & & \\
\hline
\end{tabular}

CARD 19.3: ENERGYBAL, MASSBAL

\begin{tabular}{|l|l|c|c|}
\hline $\mathbf{1 9 . 3}$ & ENERGYBAL & {$[\%]$} & {$[\%]$} \\
\hline \multicolumn{2}{|l|}{ Energy in minus energy out, normalized to energy in. Default $0.01 \%}$. \\
\hline Float & Required \\
\hline$>0.0$ & \\
\hline
\end{tabular}




\begin{tabular}{|l|l|l|l|}
\hline $\mathbf{1 9 . 3}$ & MASSBAL & {$[\%]$} & {$[\%]$} \\
\hline \multicolumn{2}{|l|}{ Mass in minus mass out, } & \multicolumn{1}{|c|}{} \\
\hline Float & Rermalized to mass in. Default $0.01 \%$. & \\
\hline$>0.0$ &
\end{tabular}

CARD 19.4: L2PRESS, L2TCOOL, L2TSOLID, L2VL, L2VV, L2VD

\begin{tabular}{|l|l|l|l|}
\hline $\mathbf{1 9 . 4}$ & L2PRESS & {$[-]$} & {$[-]$} \\
\hline Relative tolerance for the $l_{2}$ of pressure. Default 1E-5. \\
\hline Float & Required \\
\hline$>0.0$ & \\
\hline
\end{tabular}

\begin{tabular}{|l|l|c|c|}
\hline $\mathbf{1 9 . 4}$ & L2TCOOL & {$[-]$} & {$[-]$} \\
\hline \multicolumn{2}{|l|}{ Relative tolerance for the $l_{2}$ of coolant temperature. Default 1E-5. } \\
\hline Float & Required \\
\hline$>0.0$ & \\
\hline
\end{tabular}

\begin{tabular}{|l|l|l|l|}
\hline $\mathbf{1 9 . 4}$ & L2TSOLID & {$[-]$} & {$[-]$} \\
\hline Relative tolerance for the $l_{2}$ of solid temperature. Default 1E-5. & \\
\hline Float & Required \\
\hline$>0.0$ & \\
\hline
\end{tabular}

\begin{tabular}{|l|l|c|c|}
\hline $\mathbf{1 9 . 4}$ & L2VL & {$[-]$} & {$[-]$} \\
\hline \multicolumn{2}{|l|}{ Relative tolerance for the $l_{2}$ of axial liquid velocity. Default 1E-5. } \\
\hline Float & Required \\
\hline$>0.0$ & \\
\hline
\end{tabular}

\begin{tabular}{|c|c|c|c|}
\hline 19.4 & L2VV & {$[-]$} & {$[-]$} \\
\hline \multicolumn{4}{|c|}{ Relative tolerance for the $l_{2}$ of axial vapor velocity. Default $1 \mathrm{E}-5$. } \\
\hline Float & \multicolumn{3}{|c|}{ Required } \\
\hline \multicolumn{4}{|l|}{$>0.0$} \\
\hline 19.4 & L2VD & {$[-]$} & {$[-]$} \\
\hline \multicolumn{4}{|c|}{ Relative tolerance for the $l_{2}$ of axial droplet velocity. Default $1 \mathrm{E}-5$. } \\
\hline Float & Required & & \\
\hline$>0.0$ & & & \\
\hline
\end{tabular}

CARD 19.5: L2VOID, L2APRESS, L2ATCOOL, L2ATSOLID, L2AVL, L2AVV, L2AVD 


\begin{tabular}{|l|l|c|c|}
\hline $\mathbf{1 9 . 5}$ & L2VOID & {$[-]$} & {$[-]$} \\
\hline Criteria for the $l_{2}$ of void. Default 1E-5. & \\
\hline Float & Required & \\
\hline$>0.0$ & \\
\hline
\end{tabular}

\begin{tabular}{|l|l|c|c|}
\hline $\mathbf{1 9 . 5}$ & L2APRESS & {$[\mathrm{bar}]$} & [psi] \\
\hline \multicolumn{2}{|l|}{ Absolute tolerance for the $l_{2}$ of pressure. Default $0.001 \mathrm{psi}}$. \\
\hline Float & Required & \\
\hline$>0.0$ & \\
\hline
\end{tabular}

\begin{tabular}{|c|c|c|c|}
\hline 19.5 & L2ATCOOL & {$[\mathrm{C}]$} & {$[\mathrm{F}]$} \\
\hline \multicolumn{4}{|c|}{ Absolute tolerance for the $l_{2}$ of coolant temperature. Default $0.001 \mathrm{~F}$. } \\
\hline Float & Required & & \\
\hline$>0.0$ & & & \\
\hline
\end{tabular}

\begin{tabular}{|c|c|c|c|}
\hline 19.5 & L2ATSOLID & {$[\mathrm{C}]$} & {$[\mathrm{F}]$} \\
\hline \multicolumn{4}{|c|}{ Absolute tolerance for the $l_{2}$ of solid temperature. Default $0.001 \mathrm{~F}$. } \\
\hline Float & Required & & \\
\hline$>0.0$ & & & \\
\hline
\end{tabular}

\begin{tabular}{|c|c|c|c|}
\hline 19.5 & L2AVL & {$[\mathrm{m} / \mathrm{s}]$} & {$[\mathrm{ft} / \mathrm{s}]$} \\
\hline \multicolumn{4}{|c|}{ Absolute tolerance for the $l_{2}$ of axial liquid velocity. Default $0.001 \mathrm{ft} \mathrm{s}^{-1}$. } \\
\hline Float & Require & & \\
\hline$>0.0$ & & & \\
\hline
\end{tabular}

\begin{tabular}{|c|c|c|c|}
\hline 19.5 & L2AVV & {$[\mathrm{m} / \mathrm{s}]$} & {$[\mathrm{ft} / \mathrm{s}]$} \\
\hline \multicolumn{4}{|c|}{ Absolute tolerance for the $l_{2}$ of axial vapor velocity. Default $0.001 \mathrm{ft} \mathrm{s}^{-1}$. } \\
\hline Float & Require & & \\
\hline$>0.0$ & & & \\
\hline
\end{tabular}

\begin{tabular}{|l|l|c|}
\hline $\mathbf{1 9 . 5}$ & L2AVD & {$[\mathrm{m} / \mathrm{s}]$} \\
\hline \multicolumn{2}{|l|}{ Absolute tolerance for the $l_{2}$ of axial droplet velocity. Default $0.001 \mathrm{ft} \mathrm{s}^{-1}}$. \\
\hline Float & Required \\
\hline$>0.0$ & \\
\hline
\end{tabular}




\section{Output Manual}

This chapter gives an overview of output files generated by CTF. A summary of all possible output files is presented in Table 9. The table presents the file extension and a brief description of each file. The base name will depend on the base name of the input file (e.g., "deck.inp" will result in an HDF5 file called "deck.ctf.native.h5"). The table also presents the file format and usage. A "text" format file can be opened with a regular text editor. A "VTK" format file is meant to be opened with visualization software capable of reading the VTK format. The "HDF5" format is a specification managed by the HDFGroup, who also provides software for extracting file data. The Usage column of the table can have a value of either "legacy" or "current". Legacy means the file was being produced by CTF when it was adopted into CASL, but it has not been maintained or supported, so its use is cautioned against. On the other hand, Current means that the file is tested and supported and is recommended for use by the end user.

Table 9: Summary of output files produced by CTF

\begin{tabular}{|c|c|c|c|}
\hline Extension & Description & Format & Usage \\
\hline .out & $\begin{array}{l}\text { Provides input echo and channel/rod solution data or- } \\
\text { ganized into tables. }\end{array}$ & Text & Legacy \\
\hline .ctf.chans.out & $\begin{array}{l}\text { Summary of solution data for every channel in the } \\
\text { model and connected solid objects. Data are organized } \\
\text { into tables ( } 4 \text { tables per channel), including bundle av- } \\
\text { erage data ( } 2 \text { tables), or a radial average of all channel } \\
\text { data. Solution data are printed for each user-requested } \\
\text { edit. }\end{array}$ & Text & Legacy \\
\hline .ctf.gaps.out & $\begin{array}{l}\text { Summary of solution data for every gap in the model. } \\
\text { Data are organized into tables. Solution data are } \\
\text { printed for each edit. }\end{array}$ & Text & Legacy \\
\hline .ctf.chans.vtk & $\begin{array}{l}\text { Selected channel solution data in the legacy VTK for- } \\
\text { mat. This file includes mesh information and will only } \\
\text { be created when the user provides channel and gap } \\
\text { mesh data in Cards } 2.2 \text { and } 3.3 .5 \text {. The file may be } \\
\text { opened with visualization software to visualize solu- } \\
\text { tion data. }\end{array}$ & VTK & Current \\
\hline
\end{tabular}


Table 9 -Continued from previous page

\begin{tabular}{|c|c|c|c|}
\hline Extension & Description & Format & Usage \\
\hline .ctf.rods.vtk & $\begin{array}{l}\text { Selected rod solution data in legacy VTK format. Will } \\
\text { only be produced if mesh data are provided by the user } \\
\text { in Cards } 2.2 \text { and } 3.3 .5 \text { of the input deck and map data } \\
\text { are provided in Card } 17 \text {. The file may be opened with } \\
\text { visualization software to visualize solution data. }\end{array}$ & VTK & Current \\
\hline .ctf.dnb.out & $\begin{array}{l}\text { Solution data related to a DNB simulation. Data are } \\
\text { printed for each rod surface. }\end{array}$ & Text & Legacy \\
\hline$. c t f . h 5$ & $\begin{array}{l}\text { Code solution data formatted in the VERA HDF5 file } \\
\text { format. This file can be opened with VERAView. The } \\
\text { file is only produced when the user provides a core } \\
\text { map in Card } 17 \text { and is therefore only applicable for } \\
\text { pin-resolved LWR geometry. }\end{array}$ & HDF5 & Current \\
\hline .ctf.native.h5 & $\begin{array}{l}\text { This is the main output file produced by CTF. Un- } \\
\text { like the VERA HDF } 5 \text { file, it can be produced for any } \\
\text { type of model, whether it contains multiple sections, } \\
\text { lumped channels/rods, channel splitting, etc. An in- } \\
\text { terface to this file type is provided in SubKit. }\end{array}$ & HDF5 & Current \\
\hline .ctf.run.out & Code timestepping information. & Text & Legacy \\
\hline .ctf.convergence.out & $\begin{array}{l}\text { Convergence information. Only produced when mod- } \\
\text { eling a steady-state case using the old convergence } \\
\text { metrics entered on Card } 18 .\end{array}$ & Text & Current \\
\hline .ctf.newconv.out & $\begin{array}{l}\text { Convergence information. Only produced when mod- } \\
\text { eling a steady-state case using the new convergence } \\
\text { metrics entered on Card } 19 \text {. }\end{array}$ & Text & Current \\
\hline .ctf.balance.out & $\begin{array}{l}\text { Detailed mass/energy balance information in the sys- } \\
\text { tem. }\end{array}$ & Text & Current \\
\hline .ctf.pressure.out & $\begin{array}{l}\text { Detailed information on the pressure distribution in } \\
\text { the system. }\end{array}$ & Text & Current \\
\hline .ctf.mass.out & Information on the system mass balance. & Text & Legacy \\
\hline .ctf.heat.out & Information on the system heat balance. & Text & Legacy \\
\hline
\end{tabular}




\section{Users' Guide}

\subsection{General}

This section is intended to aid the user in learning to set up the input deck for CTF. There are three basic tasks the user must perform:

1. setting the geometry data to describe the system;

2. specifying the fluid conditions and forcing functions to define the state of the system; and

3. running the code and interpreting the output.

The first two tasks are covered in this section.

Any vertical one-, two-, or three-dimensional component in the reactor vessel can be modeled with CTF. The exceptions are components such as pumps or pressurizers which have special boundary conditions not included in the code capabilities. In addition, the problem must be amenable to solution by the semi-implicit numerical algorithm used.

All geometries modeled are represented as a matrix of Eulerian mesh cells. The number of cells depends on the degree of detail required to resolve the flow field, the phenomena being modeled, and practical restrictions such as computing costs and storage limitations. In two-phase flow, the mesh cell should be large compared to the characteristic size of the two-phase flow pattern. For example, the mesh cell size should be large relatively to the bubble size, so that averaged quantities (bubble size, drag, etc.) used in the calculation will be valid. In slug or film flow, the mesh cell size should be on the order of the hydraulic diameter or larger since the physical models for these flow regimes are based on physical dimensions of the flow path.

The equations for flow field in the reactor core are solved using a staggered difference scheme on the Eulerian mesh. The velocities are obtained at the mesh faces and the state variables (e.g., pressure, density, enthalpy, and phasic volume fractions) are obtained at the cell center. The mesh cell is characterized by cross-sectional area, $A$, height, $\Delta X$, and the width of the connections to the adjacent mesh cells, $S$. The basic mesh cell is shown in Figure 23.1. This cell defines the control volume for the scalar continuity and energy equations. The momentum equations are solved on a staggered mesh with the momentum cell centered on the scalar cell face. The momentum cell for axial velocities is shown in Figure 23.2 and that for transverse velocities in Figure 23.3. 
The input has been constructed to allow the user a great deal of flexibility in defining the mesh for the irregular geometries typical of reactor vessel internals. The mesh cells are defined by input in terms of subchannels. A subchannel is a vertical stack of mesh cells, as illustrated in Figure 23.4. A subchannel may represent a fluid volume between four fuel rods, a lumped region of the core, a segment of the downcomer, or any other vertical flow path appropriate to the geometry being modeled.

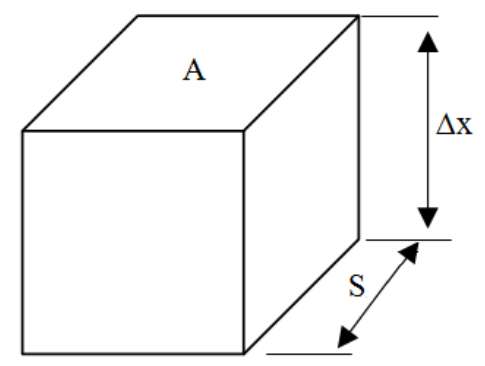

Figure 23.1: Basic mesh cell

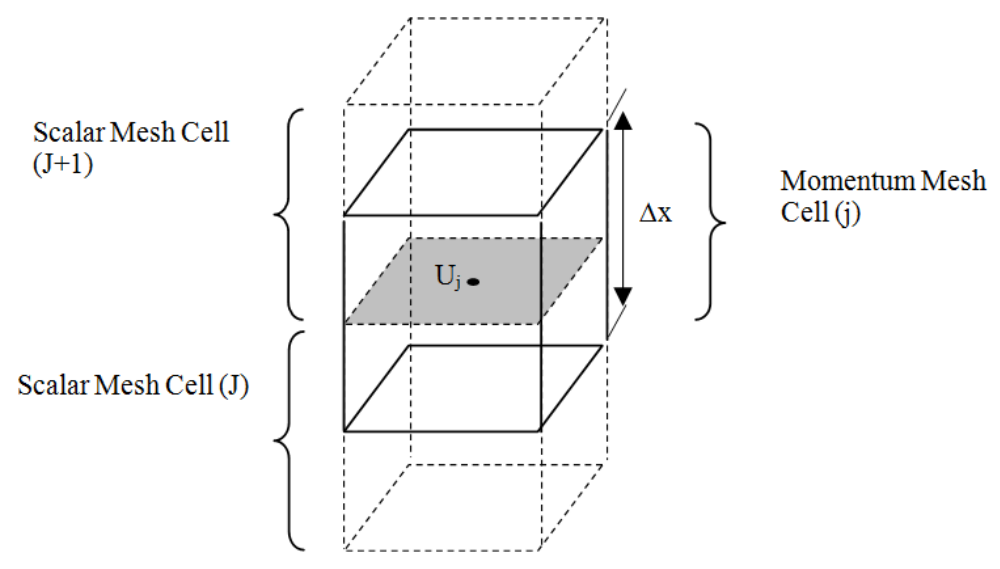

Figure 23.2: Mesh cell for axial momentum 


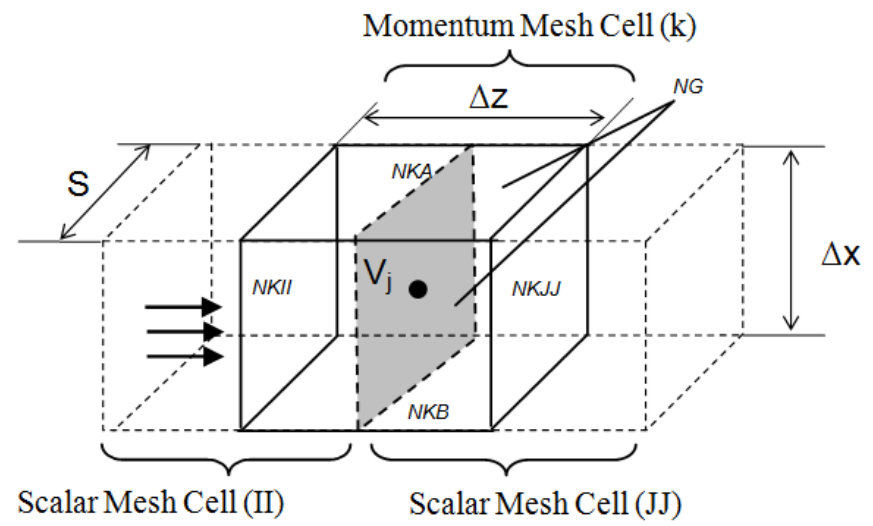

Figure 23.3: Mesh cell for transverse momentum

Boundary data for each subchannel are stored in phantom nodes at its top and bottom. Between these two phantom nodes are NDX nodes which actually enter into calculation. Node $J$ contains boundary conditions for the bottom of the subchannel and node $J=\mathrm{NDX}+2$ contains boundary conditions for the top of the subchannel. Boundaries between mesh cells are identified in Figure 23.4 with the lower case ' $\mathrm{j}$ ' and refer to locations of the momentum mesh center, where velocities are obtained. The velocity corresponding to $J=1$ is at the top of the continuity mesh cell corresponding to $J=1$.

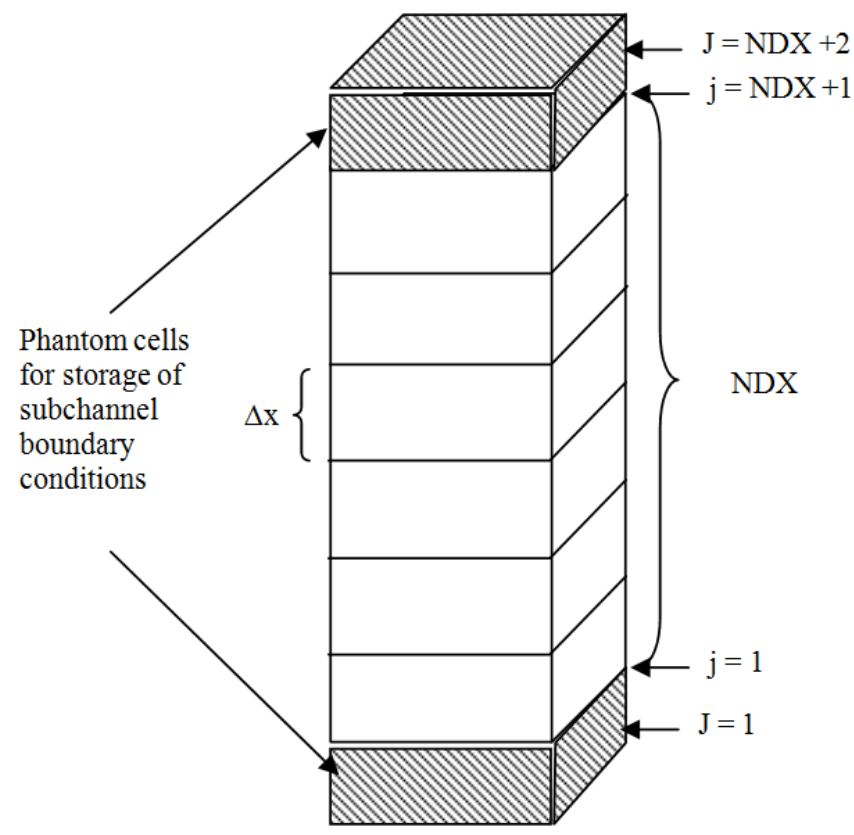

Figure 23.4: Basic Subchannel 
Nominal flow area and wetted perimeter are specified for each subchannel by input. Each mesh cell within the subchannel is assumed to have nominal geometry unless variations for area or/and wetted perimeter are specified by the user. The mesh for a particular region of the vessel is developed by pacing a sufficient number of subchannels in the region to model the geometry and the important flow phenomena of the region. Transverse connections are specified between subchannels to complete the multidimensional mesh of the region. These connections are referred to as gaps. Gaps are defined by the width of the flow path between two subchannels and the distance between the subchannels centroids. The width of the gap between subchannels is assumed to be uniform along the total length of the subchannel unless an axial variation of the gap is specified. The centroid distance is always equal to the nominal value for the gap; no variation is allowed.

All regions composed of subchannels of same vertical length and beginning at the same level in the vessel are grouped together and referred to as a section. When all sections have been defined, the sections are joined together to form the omplete mesh by specifying connections to the subchannels in adjacent sections at the top and bottom of each subchannel. The process of connecting one or more subchannels to the top or bottom of a subchannel is referred to as subchannel splitting. Each subchannel and gap in the problem is assigned a unique identification number by the input and these subchannel numbers are used to identify the connections at the top and bottom of each subchannel. Connections are not specified for subchannels without a physical flow path at the inlet or outlet. Boundary conditions on the ends of subchannels that do not connect to subchannels in an adjacent section are specified by input.

Fuel rod simulators, nuclear fuel rods and the solid structures within the vessel can be modeled using the rod and unheated conductor model. Heat transfer through solid structures, thermal storage during transient, and quenching of hot dry surfaces can be calculated by the conduction model. The user has a great deal of flexibility in modeling geometries of solid structures in the system. They can be represented as cylindrical rods, hollow tubes, or flat walls composed of any number of different materials with specified thermal properties.

To summarize, the basic building block for the vessel mesh is the subchannel, which is a vertical stack of single mesh cells. Several subchannels can be connected together by gaps to model a region of the reactor vessel. Regions that occupy the same level form a section. Sections are connected axially to complete the vessel mesh by specifying subchannel connections between sections. Heat transfer surfaces and solid structures that interact significantly with the fluid can be modeled with rods and unheated conductors.

\subsection{Specification of the Geometry Data}

This section provides the user with guidance how to model the geometry of the problem being simulated. It covers the code input requirements for modeling of the subchannels and gaps, the splitting of axial sections, and the spacer grid data.

\subsubsection{Instructions to CARD GROUP 2}

The input for CARD GROUP 2 specifies the number of subchannels in the model and their geometrical characteristics. The total number of subchannels, NCHANL, is specified on CARD 2.1. Each subchannel is uniquely identified on CARD 2.2 by an index number I, flow area AN (I), and wetted perimeter PW(I). The 
subchannel identification numbers are completely arbitrary and the user is not required to number them sequentially. The only constrain on subchannel numbers is that they must be unique.

The variables ABOT and ATOP on CARD 2.2 are optional input to model the area at the top and the bottom of the subchannel for the momentum equation solution. By default both are equal to the subchannel nominal flow area AN(I). When at the section boundaries there are sudden changes in the geometry of the system (for example, orifice or flow distribution plates), ABOT and ATOP are used to supply the correct area at the bottom and the top of the subchannel for the momentum solution. Nonzero values for ABOT and ATOP will replace the nominal subchannel area at those locations.

If the axial velocities for a subchannel can convect transverse momentum between sections, the location of those velocities must be specified by the user. If this input is not provided, transverse momentum will accumulate in the affected cells of subchannel I at the section boundaries, causing errors in the pressure solution. The number of gaps for which subchannel I convects transverse momentum between sections is specified as the variable NAMGAP on CARD 2.2.

The connections for axial convection of transverse momentum across the section boundary by a given subchannel I are specified on CARD 2.3. The connections are defined by the node number INODE(I) of the axial velocity convecting momentum and the index numbers of the gaps, above and below the section boundary, whose momentum is convected. The node numbering convention is shown in Figure 23.5. The center of the scalar mesh are identified by $J=1,2, \ldots($ NONODE +2$)$ and the centers of the momentum cells are identified by $J=1,2, \ldots(\operatorname{NONODE}+1)$. The node number INODE $(\mathrm{I}, \mathrm{N})$ will be 1 for the subchannel velocity at the bottom of the boundary of the section, or NONODE +1 for the subchannel velocity at the top of the section. KGAPB ( I, N) is the gap number on the lower side of the section boundary and KGAPA (I,N) is the gap number of the upper side of the section boundary. If the flow is positive, the axial velocity of subchannel I convects transverse momentum from KGAPB to KGAPA. If the flow reverses, momentum is convected from KGAPA to KGAPB. This input is fairly obvious for cases where there is a gap both above and below the section boundary. But even if one or other is nonexistent this input must be provided to tell the code how to dissipate transverse momentum that is transported out of a gap across a section boundary. If there is a gap below, but no gap above the section boundary, KGAPA is entered as zero and momentum convected out of KGAPB is considered dissipated. Similarly, if there is a gap above, but no gap below, KGAPB is entered as zero. Reverse flow convects momentum out of KGAPA, which is considered dissipated. The area through which momentum is convected is calculated as the minimum of the $S_{k} L_{k} / 2$ for KGAPA and KGAPB and the flow area of subchannel I. A subchannel can convect transverse momentum to or from several gaps at the top and/or bottom of the section. For user convenience, when only one axial section is modeled, the input values of CARD 2.3 are ignored. They are created automatically by the code; which means NAMGAP(I) may be set to zero and CARD 2.3 may be omitted.

In defining the input for CARD 2.3, the user must understand how the momentum equation is solved at the section boundaries, to determine if the axial velocities will be solved in subchannel $I$ at the section boundary. It does not do any good to specify the NAMGAP connections for subchannels that are not solved at the section boundary, and conversely, they must be specified for those that are. Regardless of the geometry being modeled, there are three basic patterns for subchannel splitting connections. These are:

1. one subchannel below connected to one subchannel above (see Figure 23.6 case (1));

2. one subchannel below connected to many subchannels above (see Figure 23.6 case (2)); and

3. multiple subchannels below connected to one subchannel above (see Figure 23.6 case (3)). 


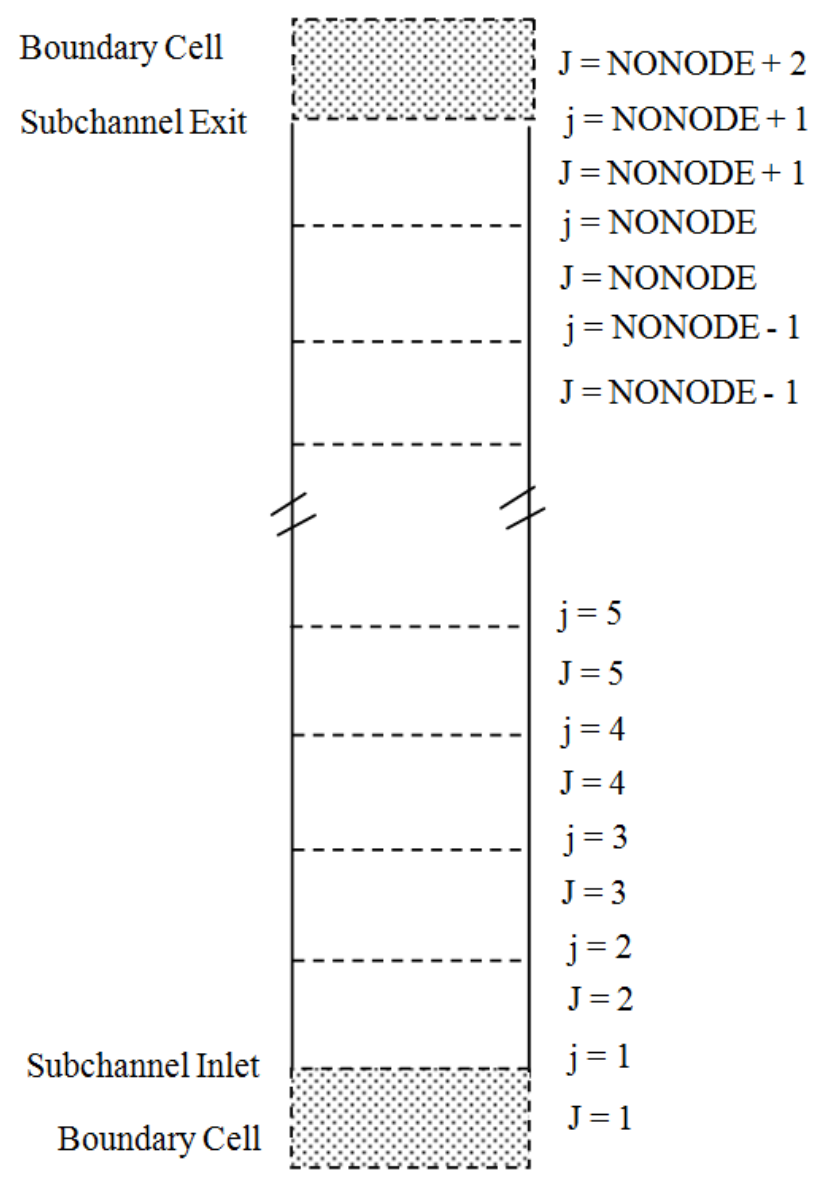

Figure 23.5: Subchannel node numbering convention 

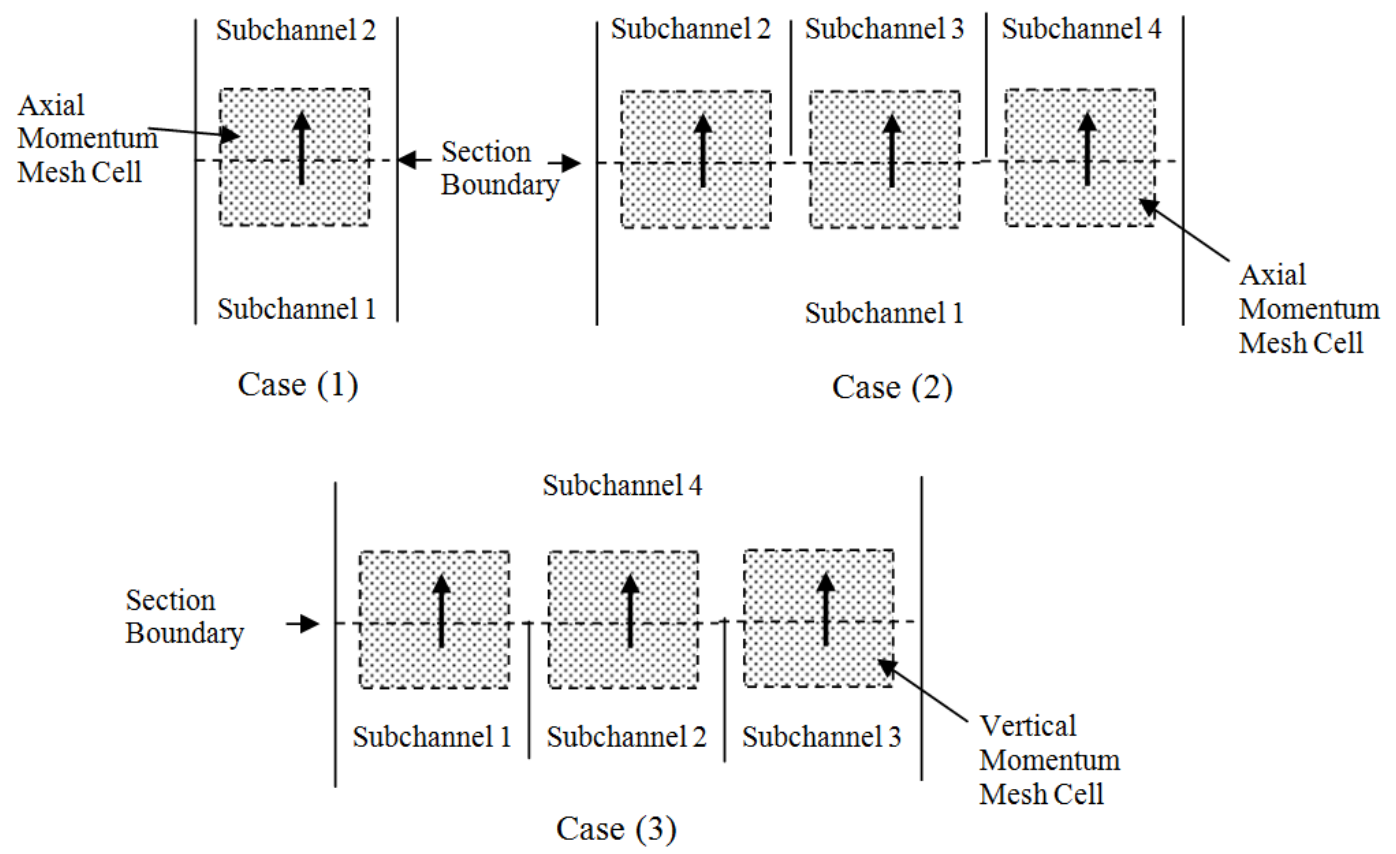

Figure 23.6: Subchannel connections at section boundaries allowed by the subchannel splitting logic

Transverse momentum convection input data are required only for subchannels where the momentum equation will be solved at the section boundary, and velocities so obtained can convect transverse momentum from or to adjacent gaps. The momentum equation is solved at the top of subchannel 1 for case (1) and is not solved at the bottom of subchannel 2. The momentum equation is solved at the bottom of subchannels 2,3 , and 4 in case (2), and at the top of subchannels 1,2 , and 3 in case (3).

The possible subchannel and gap configurations at section boundaries are outlined in Figure 23.7. The case where two subchannels below the section boundary connect to two subchannels above the section boundary with no change in the number of mesh cells between sections is illustrated in Figure 23.7 (a). The axial momentum equation is solved in the top nodes of subchannels 1 and 2 to obtain axial velocities at the section boundary. The velocity of subchannel 1 convects transverse momentum from the left side of the transverse momentum cell for gap 1 to the left side of the transverse momentum cell for gap 2. Similarly, the velocity of subchannel 2 convects momentum from the right side of the momentum cell for gap 1 to the right side of the momentum cell for gap 2. For this example, the input for CARD 2.3 must be supplied for both subchannels 1 and 2 as follows: 


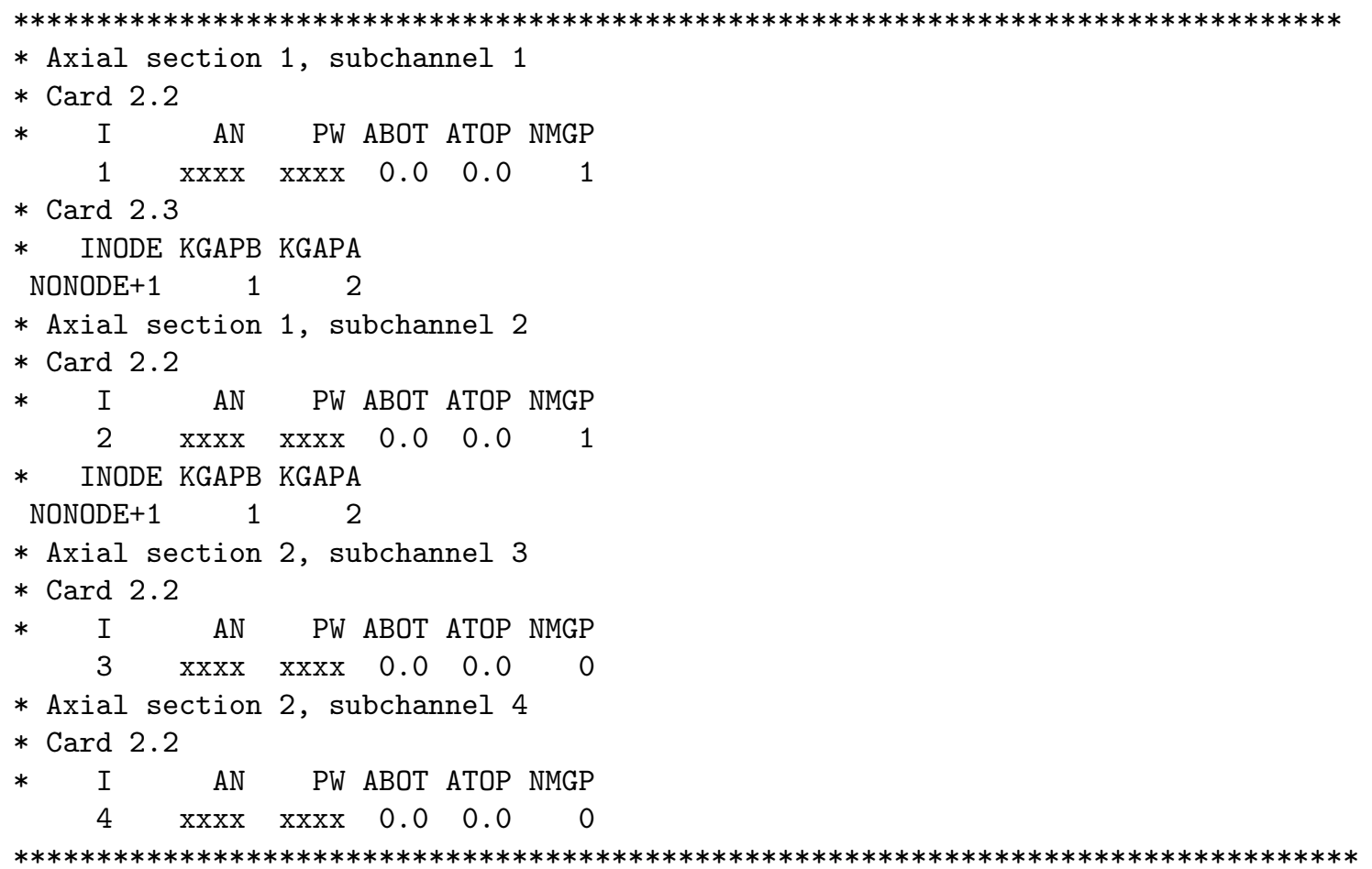

For the case shown in Figure 23.7 (b), there is no gap on the top side of the section boundary, so the axial velocities at the top of subchannels 1 and 2 convect momentum out of gap 1, but the momentum is assumed to be dissipated since there is no transverse momentum cell to receive it. For this case, the input will be:

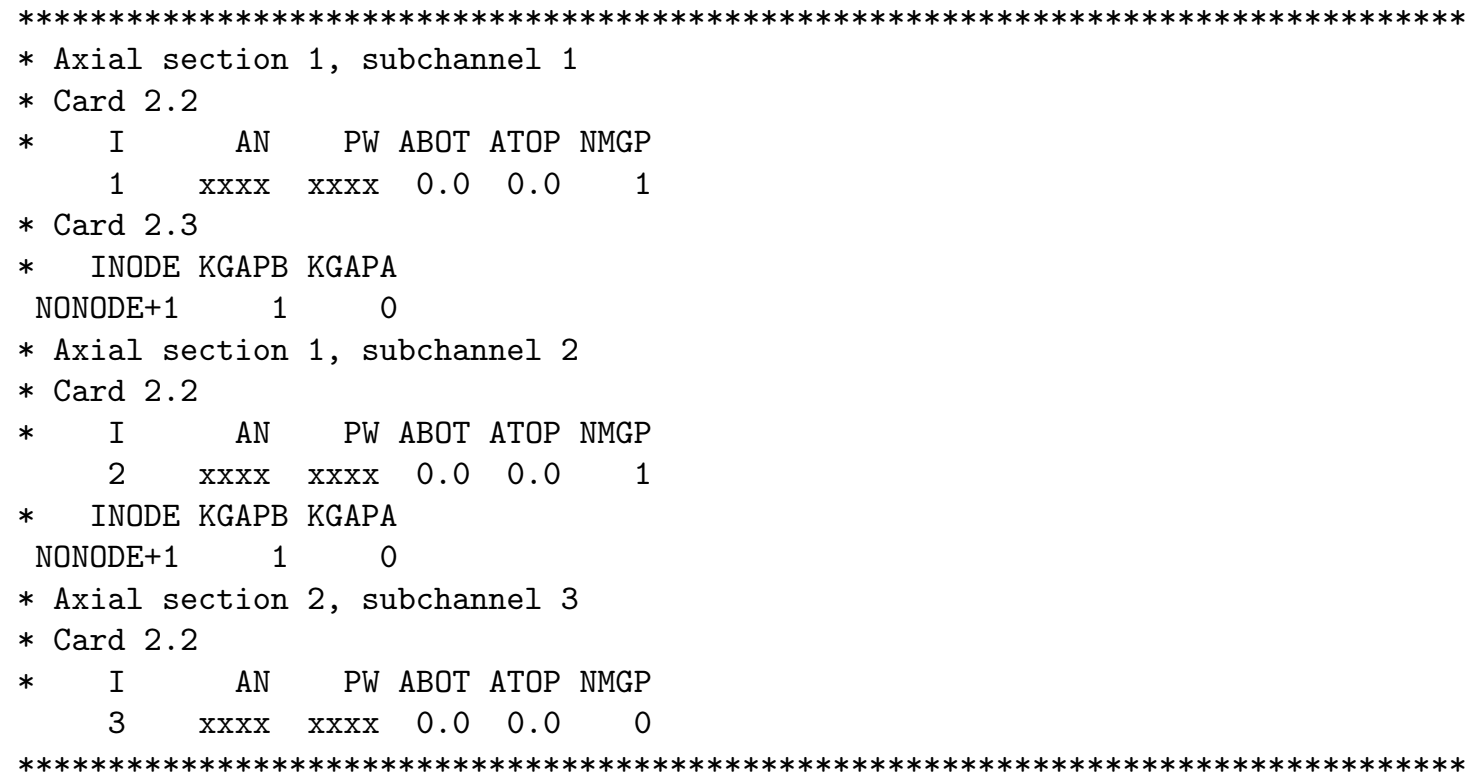


If a gap exists both above and below the section boundary as in Figure 23.7 (a), but there is a flow straightener (such as a core support plate) at the section boundary, then the transverse momentum can be dissipated by specifying two connectors for each subchannel, one for positive flow and the other for negative flow. Positive flow convects momentum from gap 1 and dissipates it; negative flow convects momentum from gap 2 and dissipates it:

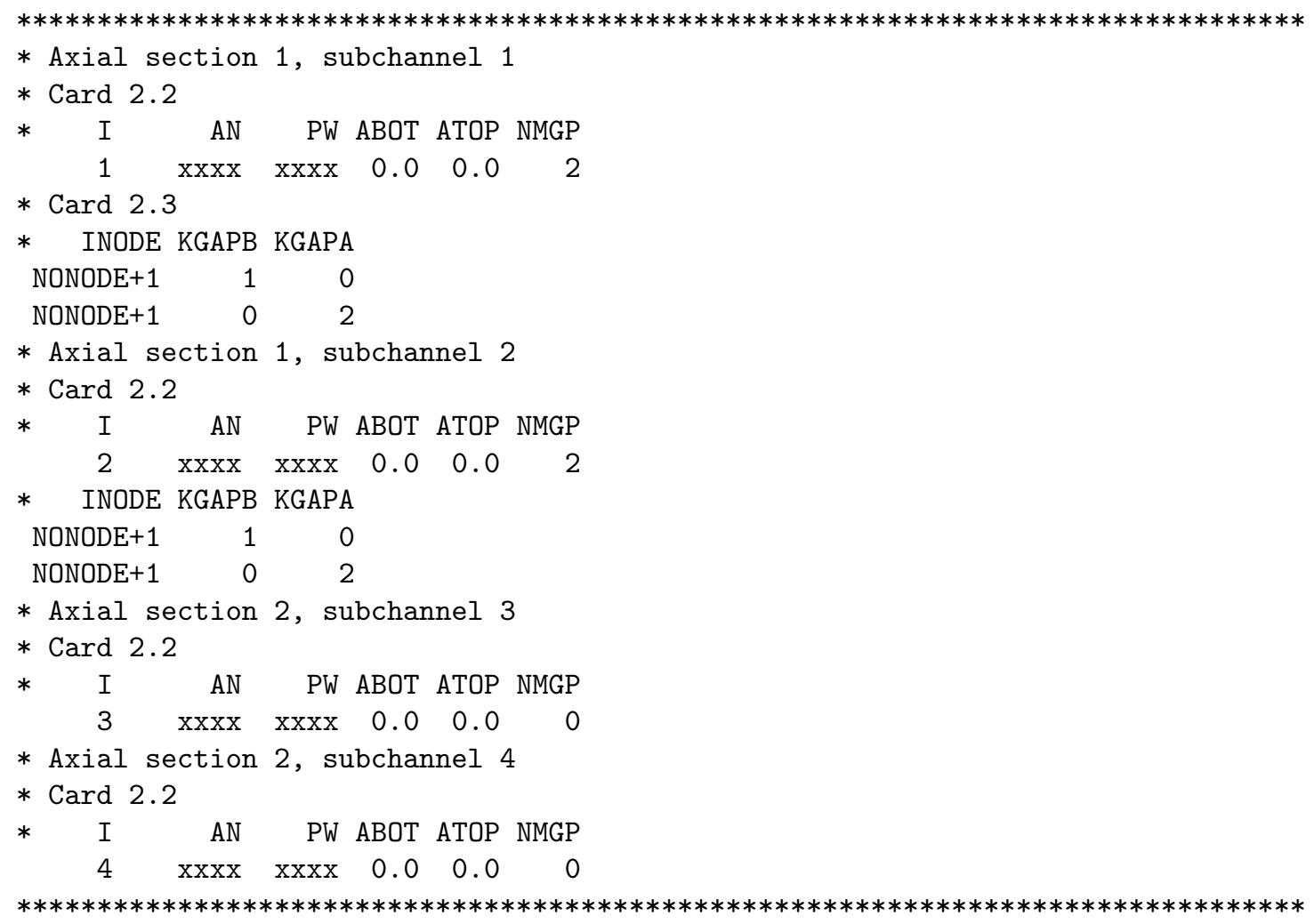

If momentum is convected by axial velocity from one gap on one side of the section boundary to several gaps on the other side (variable mesh) then transverse momentum convection connections must be specified for each gap. The momentum taken from the single gap is apportioned among the connecting gaps by the relative size of the areas through which the momentum is convected.

\subsubsection{Instructions to CARD GROUP 3}

The gap input defines the control volume for the transverse momentum equation, on either a subchannel analysis basis or for a three-dimensional analysis of transverse flow.

The total number of gaps, NK, is read on CARD 3.1. The geometry data for each gap is read on CARD 3.2. Each gap is identified by a unique number $K$. Gap $K$ connects subchannel IK (K) to subchannel JK(K). By convention, IK $(K)$ is the lower-numbered subchannel of the pair, JK $(K)$ is the higher-numbered subchannel of the pair, and the crossflow through the gap $K$ is positive when passing from IK (K) to JK(K). Flow the 


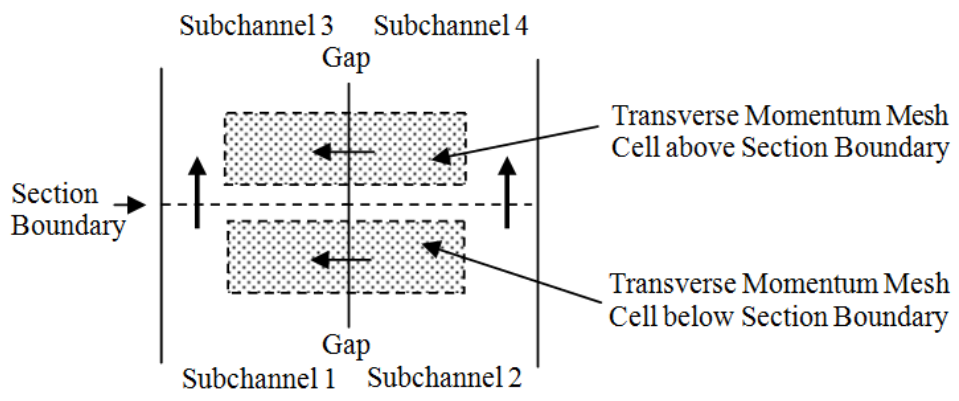

(a)

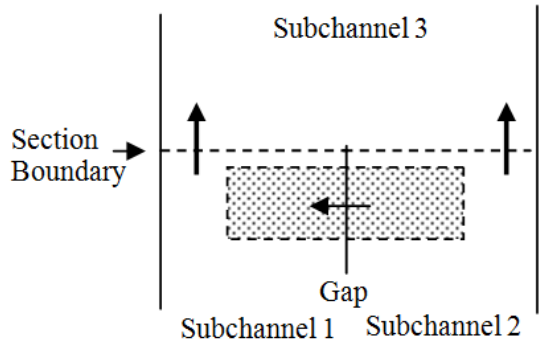

(b)

Figure 23.7: Typical configuration for convection of transverse momentum between sections

other way is negative. The nominal gap width is specified as GAPN $(K)$ and the distance between the centers of the connected subchannels is LENGTH(K). GAPN(K) and LENGTH(K) define the width and length of the transverse momentum mesh cell. The flow area between the connecting subchannels is given by the product of the gap width and the axial length increment for the mesh, DXS (to be read on CARD 4.2). The gap width, GAPN $(K)$, is equal to the total width for transverse flow between the two adjacent regions modeled by the subchannels IK $(K)$ and JK(K).

Form and wall drag in gap $K$ is specified for the transverse momentum equations using the parameters WKR $(K)$ and FWALL $(K)$.

The form drag loss coefficient (velocity head), WKR(K), is specified by the user. The value of WKR(K) for a gap depends on the geometry of the flow path modeled. A value of 0.5 is typically used for flow across one row of rods or tubes. For lumped subchannels, this value is multiplied by the number of rod rows the gap momentum cell contains.

Wall friction in the gap is ordinarily included in the WKR parameter, but for gaps that are formed by vessel walls rather than rod arrays (as in the downcomer annulus, for example) the pressure loss in the gap is primarily a friction loss rather than a form loss and should be modeled accordingly. Wall friction factors in a gap are computed internally in the code according to the user-specified value of the FWALL (K) parameter. If FWALL(K) is zero, no wall friction is calculated (gap formed by a rods only). If FWALL(K) is set equal to 0.5 , a wall with a surface of DXS $*$ LENGTH $(K)$ is assumed (one side of the gap control volume is a solid wall). If FWALL (K) is set equal to 1.0, a wall with a surface of $2 *$ DXS $*$ LENGTH $(K)$ is assumed (a solid wall on each side of the gap control volume). Both a form loss and a wall friction factor can be specified for a gap. One 


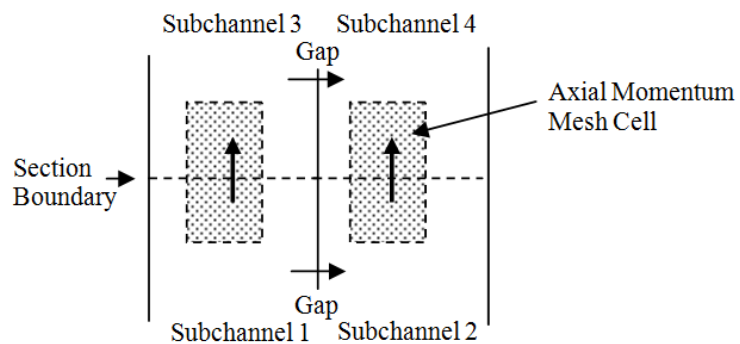

Figure 23.8: Axial momentum mesh cell at section boundary

or the other can be set to zero depending of the geometry of the problem. Both can be set to zero if the gap is in an open fluid region where the fluid-to-fluid shear within the continuous phase adequately models the transverse drag forces.

The input required to describe transverse convection of axial momentum between gaps across section boundaries employs the same sort of reasoning as does the input in CARD GROUP 2 to describe the axial convection of transverse momentum across section boundaries. The user must specify which transverse gap velocities can transport axial momentum into or out of the subchannels associated with gap $K$ at the top and bottom of the section. The transverse velocity of gap IGAPB (K) (at node NONODE +1 in the section below) can convect axial momentum into or out of the bottom cell of the subchannels associated with gap $K$. The transverse velocity of gap IGAPA(K) (at node 2 in the section above) can convect axial momentum into or out of the top cell of the subchannels associated with gap $K$. Transverse convection of axial momentum at a section boundary is illustrated in Figure 23.8. In this example, axial momentum is convected between the two axial mesh cells by the transverse velocity of gap 1 below the section boundary and by the transverse velocity of gap 2 above the section boundary. The input for IGAPA(K) identifies the gap in the section above gap $K$ that convects axial momentum between the axial momentum cells associated with the top of gap $K$. For the example shown in Figure 23.8, IGAPA $(\mathrm{K})=2$ and the corresponding input will have the following format:

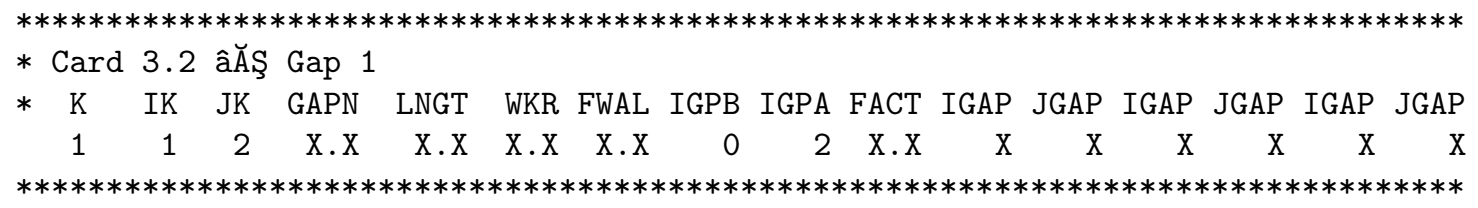

The remainder of the input for CARD 3.2 is required only for the three- dimensional form of the transverse momentum equation. If the subchannel formulation is desired for a specific problem, this input is omitted. It is used to define consistent transverse flow directions for the global coordinate system and to set up connections for orthogonal transport of momentum.

The normal crossflow sign convention is not always congruent with a global coordinate system. The user can convert the local transverse flow sign convention to an appropriate global system by specifying the variable FACTOR $(\mathrm{K})$ to indicate the orientation of gap $K$ in the global coordinate system. Figure 23.9 (a) gives two examples how FACTOR can be used. Example (a) shows an annular subchannel arrangement typically used in the downcomer. The annular flow subchannels are two-dimensional, so it is only necessary to define 
a transverse direction that is consistently positive. The normal convention for defining positive crossflow results in crossflow from subchannel 1 to subchannel 2 and from subchannel 3 to subchannel 4 is to have both being positive. Assuming that the clockwise direction is chosen as positive for the global coordinate system, if flow from IK $(K)$ to $\mathrm{JK}(K)$ is clockwise, then FACTOR $(K)$ is set to 1.0. If flow from IK $(K)$ to JK(K) is counterclockwise, the sign convention must be reversed by setting FACTOR(K) to -1.0. In this example, FACTOR (2) is set to -1.0 and the input will have the following format:

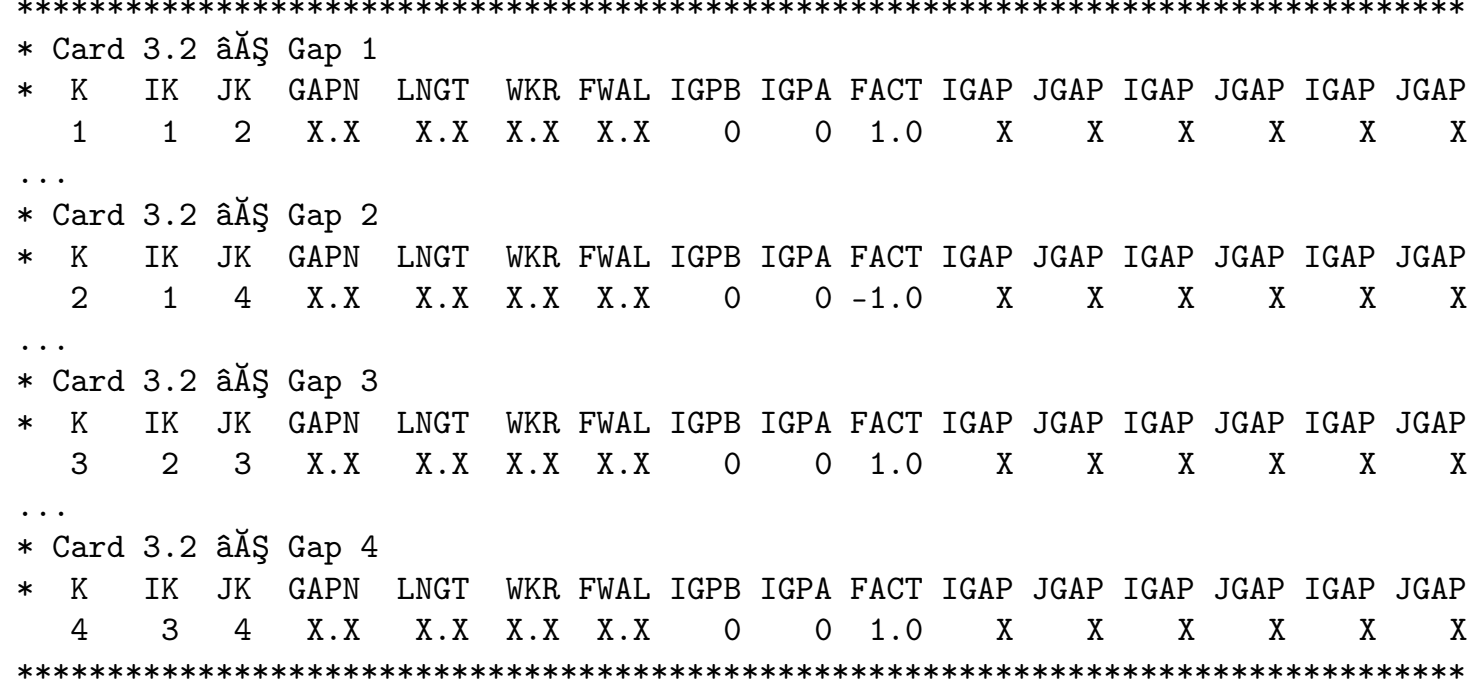

A three-dimensional subchannel array is shown in example (b) in Figure 23.9. For this case, the values of FACTOR $(\mathrm{K})$ must correspond to the actual orientation of the velocity vector in the gap on the global coordinate system. The arrows in the gaps indicate the positive crossflow direction defined by the normal convection. For gaps 1, 3, 6, and 7, this is the same as the global coordinates, so FACTOR (1), FACTOR(3), FACTOR (6), and FACTOR (7) are 1.0 for this case. But the positive flow direction in gaps 2 , 4, and 5 is exactly opposite of the global convention, so FACTOR(2), FACTOR(4) and FACTOR(5) must be specified as -1.0 to reverse the sign on the crossflow velocity. The input will have the following format: 


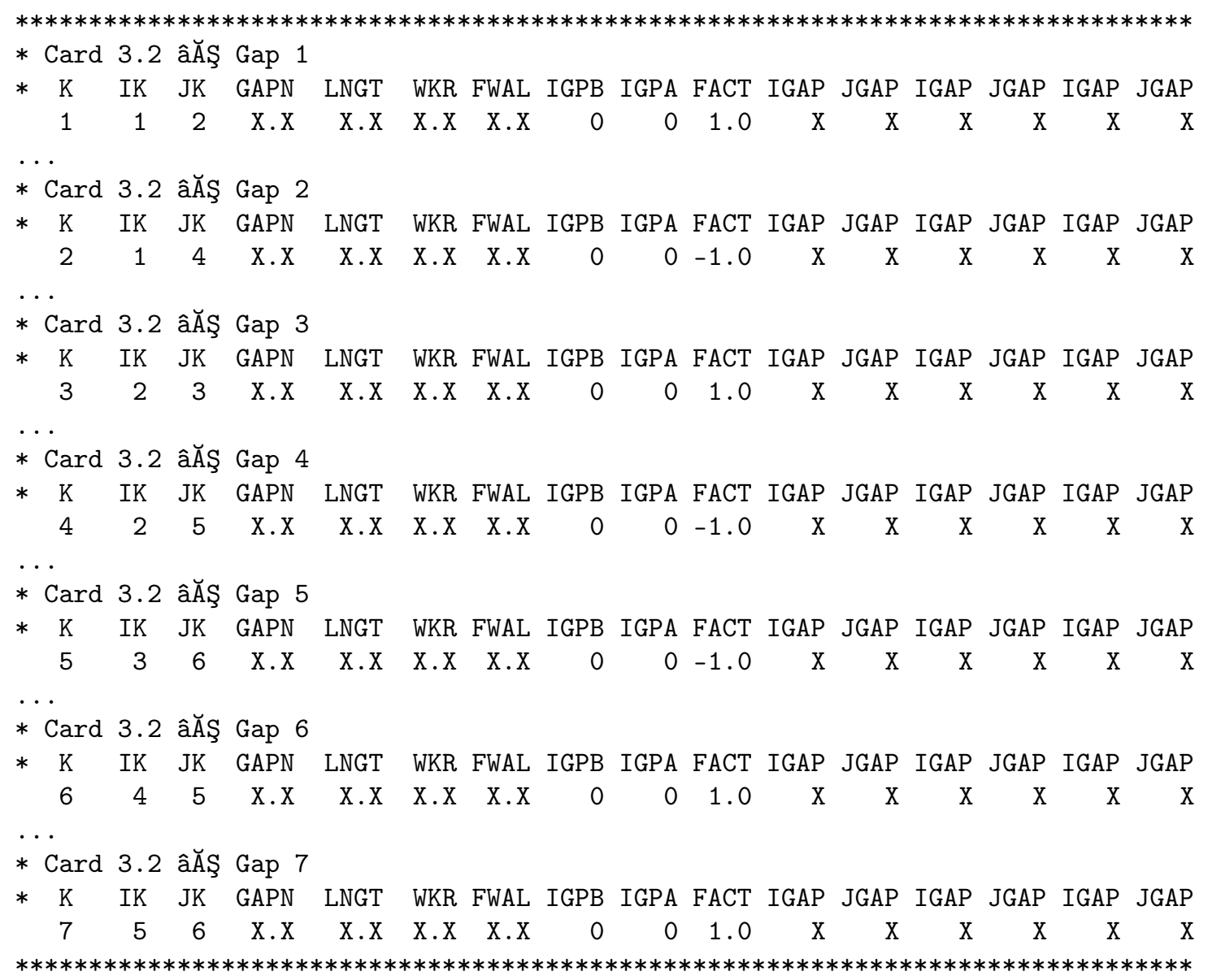

The user must specify the gaps facing a given gap $K$ in order for the code to calculate the normal components $\left(\rho V^{2} A\right)$ of transverse momentum. The arrays $\operatorname{IGAP}(\mathrm{K}, \mathrm{N})$ and JGAP $(\mathrm{K}, \mathrm{N})$ on CARD 3.2 are used to supply this data. The IGAP array holds the identification numbers of up to three gaps on the IK (or lower-numbered subchannel) side of gap $K$. The JGAP array holds the identification numbers of up to three gaps on the $J K$ (or higher-numbered subchannel) side of gap $K$. This is illustrated in Figure 23.10. In this example, gap 4 connects subchannels 1 and 2, with the conventional positive flow direction shown by the arrow. Gaps 1 and 3 face the $I K$ side of gap 4 , and gaps 5 and 6 face the $J K$ side. The input will have the following format: 


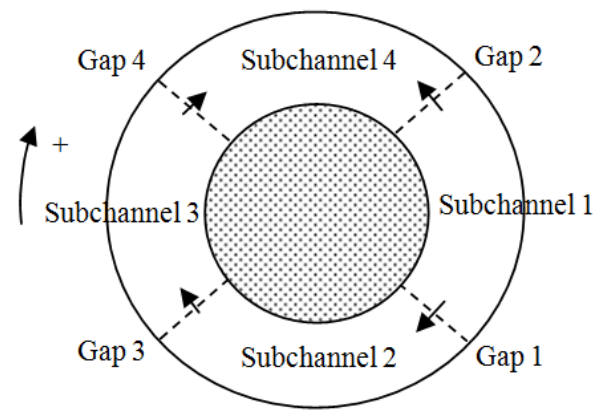

(a)

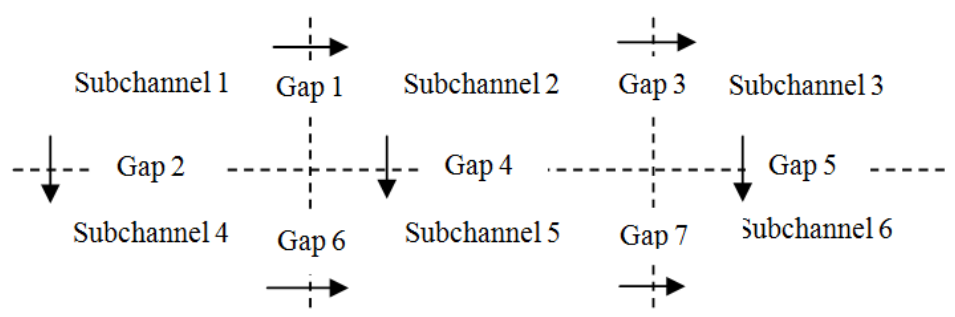

(b)

Figure 23.9: Global coordinate systems

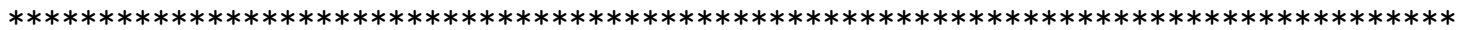

* Card 3.2 âĂŞ Gap 1

* $\mathrm{K}$ IK JK GAPN LNGT WKR FWAL IGPB IGPA FACT IGAP JGAP IGAP JGAP IGAP JGAP

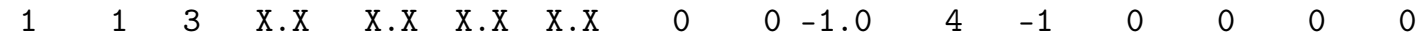

$\cdots$

* Card 3.2 âĂŞ Gap 2

* $\mathrm{K}$ IK JK GAPN LNGT WKR FWAL IGPB IGPA FACT IGAP JGAP IGAP JGAP IGAP JGAP

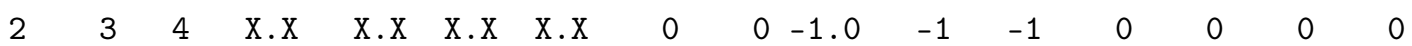

...

* Card 3.2 âĂŞ Gap 3

* $\mathrm{K}$ IK JK GAPN LNGT WKR FWAL IGPB IGPA FACT IGAP JGAP IGAP JGAP IGAP JGAP

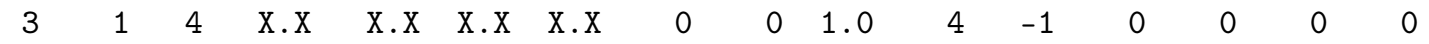

...

* Card 3.2 âĂŞ Gap 4

* $\mathrm{K}$ IK JK GAPN LNGT WKR FWAL IGPB IGPA FACT IGAP JGAP IGAP JGAP IGAP JGAP

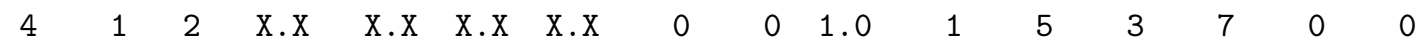

...

* Card 3.2 âĂŞ Gap 5

* $\mathrm{K}$ IK JK GAPN LNGT WKR FWAL IGPB IGPA FACT IGAP JGAP IGAP JGAP IGAP JGAP

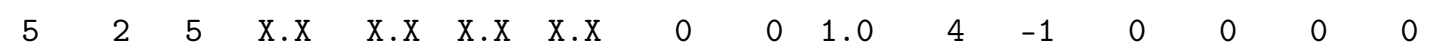

...

* Card 3.2 âĂŞ Gap 6

* $\mathrm{K}$ IK JK GAPN LNGT WKR FWAL IGPB IGPA FACT IGAP JGAP IGAP JGAP IGAP JGAP

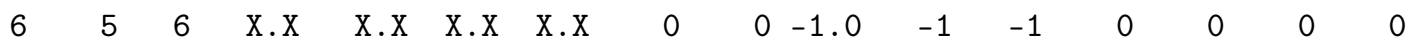

...

* Card 3.2 âĂŞ Gap 7

* $\mathrm{K}$ IK JK GAPN LNGT WKR FWAL IGPB IGPA FACT IGAP JGAP IGAP JGAP IGAP JGAP

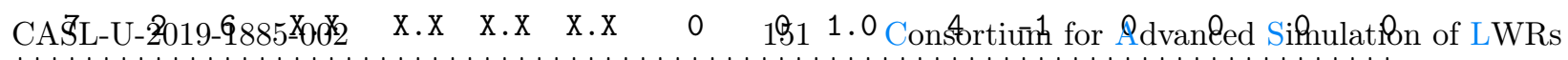


Gap 4

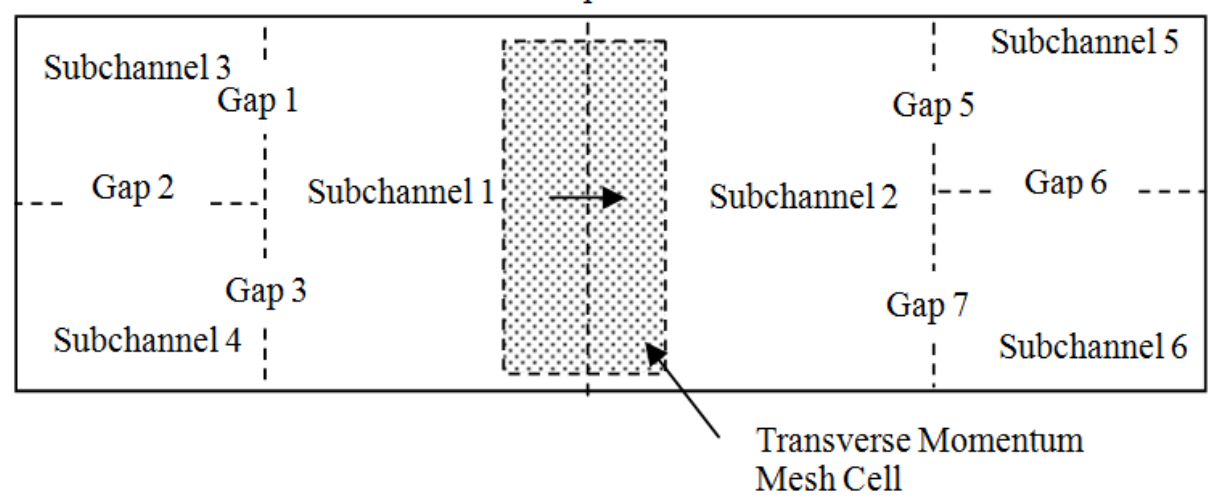

Figure 23.10: Axial momentum mesh cell at section boundary

The gap numbers are required for the three-dimensional form of the transverse momentum equation, as well as in certain two-dimensional flow systems, as illustrated in Figure 23.11. In this 2D lateral pipe flow example, the flow enters axially and accelerates into the pipe, leading to a lateral pressure drop, and recovers pressure at the outlet while decelerating out of the pipe. In order for the code to calculate the acceleration and deceleration pressure drops, the user must specify the gap numbers for all the gaps, with the IGAP for gap $1(I K=1$ and $J K=2)$ and JGAP for gap n-1 $(I K=\mathrm{n}-1$ and $J K=\mathrm{n})$ set to zero. If the user specifies a mass source at the first cell $(\mathrm{J}=2)$ in this example, then IGAP for the first gap will be $-(40+I K=1)=-41$. If a mass source boundary condition is specified in this way, then there will be no acceleration pressure drop at the beginning of the pipe. Note that it is permissible for the user to enter 0 for the IGAP and JGAP inputs; however, it will not be possible for CTF to correctly calculate the acceleration/deceleration pressure drops, as no lateral momentum will be transported into or out of the cell.

CARD 3.3 must be read for each gap, following CARD 3.2. This input is designed to model gaps between subchannels that model large regions in the vessel. The nominal gap width, GAPN (K) (supplied on CARD 3.2), is the total width for crossflow between subchannels. The characteristic width for two-phase flow through the gap, however, is the distance between the physical structures and not necessarily the total width of the connection between mesh cells. The parameter GMULT(K), input on CARD 3.3, is the number of uniform spaces between structures in the region modeled by the gap. The characteristic dimension for two-phase flow is given by:

$S^{\prime}=\frac{\operatorname{GAPN}(\mathrm{K})}{\operatorname{GMULT}(\mathrm{K})}$

The dimension $S^{\prime}$ determines the maximum bubble size that can pass between structures. If GAPN (K) is the actual width available for flow, GMULT (K) is set to 1 .

$\operatorname{ETANR}(\mathrm{K})$, which is also read on CARD 3.3, is the fraction of transverse droplet flow de-entrained on structures within the mesh cell. In a reactor vessel or test section the array of rods in the core and support columns and guide tubes in the upper plenum can contribute significantly to de-entrainment of droplets from the transverse component of the flow. The de-entrainment fraction, ETANR(K), for a gap modelling an array of tubes is:

$\operatorname{ETANR}(\mathrm{K})=1-\left(1-\eta_{R}\right)^{N}$ 


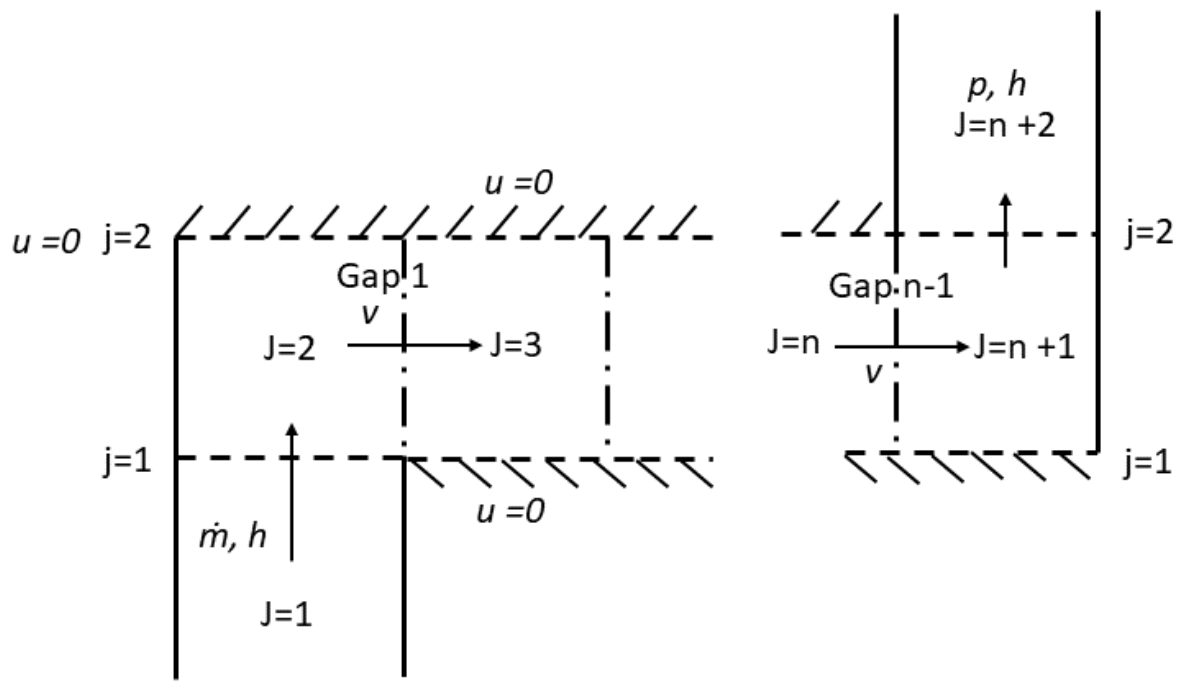

Figure 23.11: Lateral pipe flow case with axial mass flow inlet and pressure outlet

where $\eta_{R}$ is the de-entrainment fraction of a single row of tubes and $N$ is the number of tubes. The de-entrainment fraction of a single row of tubes is:

$\eta_{R}=\eta_{I}\left(1+4.5 \beta^{2}\right)$

where $\eta_{I}=0.19$ for cylindrical tubes and $\eta_{I}=0.27$ for square tubes. $N$ is the pitch-to-diameter ratio of the array.

$\operatorname{ETANR}(\mathrm{K})$ can be used to model the crossflow de-entrainment rate for geometries other than tube banks and rod bundles, but the user must determine the appropriate value for each particular application.

CARDS 3.4 and 3.5 are required only for the three-dimensional form of the transverse momentum equation. The total number of gaps that convect orthogonal transverse momentum is specified as the NLMGAP parameter on CARD 3.4. The velocity of the gap identified in KGAP1 (N) convects transverse momentum from KGAP3 (N) to KGAP2 (N) if the velocity is positive and from KGAP2 (N) to KGAP3(N) if negative. This is illustrated in Figure 23.12. In this example, the velocity of gap 1 convects momentum from the left half of the momentum mesh cell for gap 4 to the left half for gap 2. The velocity of gap 3 convects momentum from the right half of the momentum mesh cell for gap 4 to the right half for gap 2. Gaps 2 and 4 also convect momentum from gap 1 to gap 3 . Then, $\operatorname{KGAP} 1(1)=1 ; \operatorname{KGAP} 2(1)=2 ; \operatorname{KGAP} 1(2)=3 ; \operatorname{KGAP} 3(2)=4 ; \operatorname{KGAP} 1(3)=2$; $\operatorname{KGAP} 2(3)=3 ; \operatorname{KGAP} 3(3)=1 ; \operatorname{KGAP} 1(4)=4 ; \operatorname{KGAP} 2(4)=3 ;$ and $\operatorname{KGAP} 3(4)=1$. The input will have the following format: 


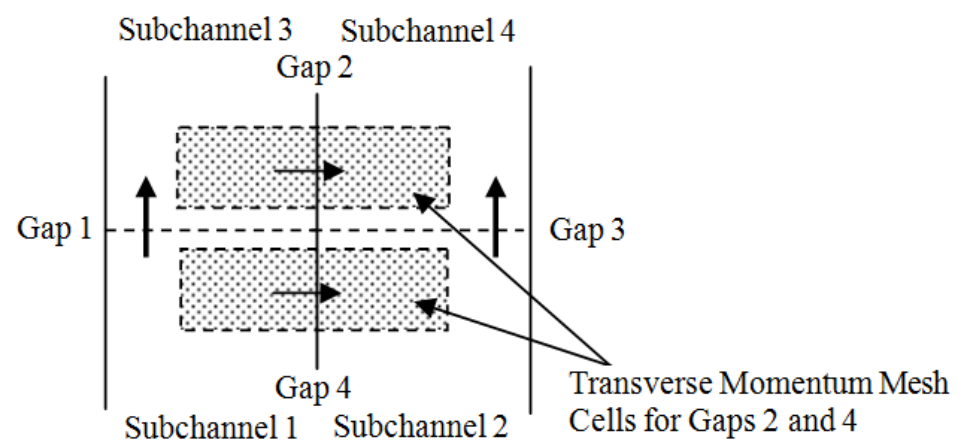

Figure 23.12: Convection of transverse momentum by an orthogonal transverse velocity

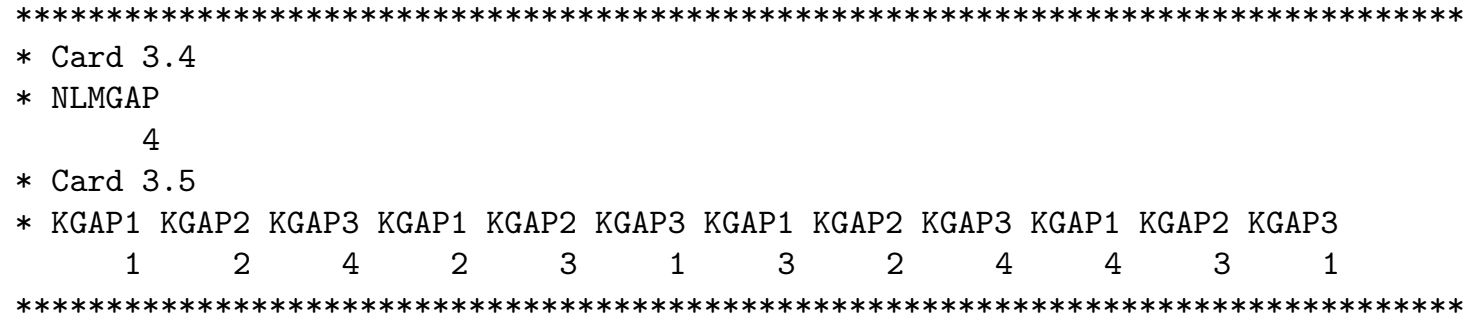

In general, the convection of transverse momentum by transverse velocities in the gap between local and global (lumped) mesh cells is neglected. The transverse flow between local mesh and global mesh is assumed to be axisymmetric. The shape of the transverse momentum cell is idealized and requires the user's judgment in selecting values for the gap width and length. The gap width should be chosen such that the product of the gap width and the axial mesh length increment is equal to the physical area for the flow path between the local and global mesh cells. The shape of the momentum mesh cell is assumed rectangular and its length should be chosen to give a physically meaningful time constant for accelerating flow through the gap. A distance equal to the diameter of the local mesh is recommended for the centroid distance (LENGTH(K)).

Setting up the gap geometry data is relatively simple even for peculiar geometries if the user bears in mind that GAPN (K) and LENGTH(K) define the transverse control volume for the gap. The size and shape of such a control volume should bear some logical resemblance to the physical structure being modeled, within the constraints of the nodding philosophy used in the code. The control volume is important not only for defining the location and magnitude of the transverse flow, but also for determining axial transport of transverse momentum through the top and bottom surfaces of the control volume. Both aspects of the momentum solution must be considered in defining the gap width and centroid length.

\subsubsection{Instructions to CARD GROUP 4}

The input data for CARD GROUP 4 describes the axial subchannel connections between sections and defines the rebalancing and simultaneous solution group options.

CARD 4.1 provides the input for the total number of axial sections in the problem, NSEC; the total number 
of the simultaneous solution groups, NSIM, in the iterative pressure matrix solution; and the flag IREB for selection of the rebalancing option for enhancement of the convergence rate for the iterative solution (parameters NSIM and IREB will be discussed later in the text).

CARD 4.2 provides the input data describing each axial section in the problem. It contains the section number, ISEC; the number of subchannels, ICHN, in the section; the number of axial nodes, NONODE, in the section; the axial node length, DXS, in the section; and the flag IVARDX for variable node length in the section. The section boundaries are uniform at a given axial level, so all subchannels within the section have the same total length. The axial node length DXS defines the length of both the continuity and momentum control volumes. DXS is constant within the section and therefore the total axial length of the section is DXS $*$ NONODE. DXS can vary between sections and each section may have one or many axial nodes. The only constraint is that the change in DXS between adjacent sections should not be greater than factor of two.

CARD 4.3 specifies a variable axial node length within a section. The input data contains the last axial level JLEV (I) in a section to have a node length of VARDX (I), where $I$ is the number of pairs to be read and it is equal to the parameter IVARDX read on CARD 4.2. For the example shown in Figure 23.13, the input will have the following format:

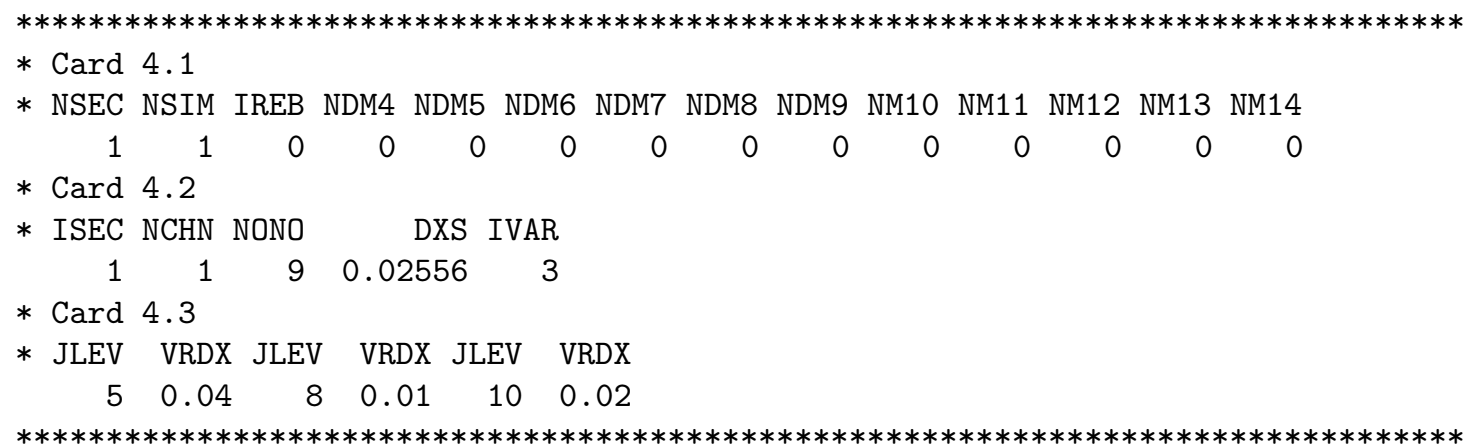

CARD 4.4 specifies the axial connections for each subchannel in a section. A subchannel may connect up to six subchannels in the section above and six subchannels in the section below. The array KCHANA contains the index numbers of subchannels connected to the top of the subchannel and the array KCHANB contains the index numbers of the subchannels connected to the bottom. If a subchannel does not have a connection above or below, KCHANA or KCHANB is specified with the subchannel's own identification number. The code will not accept a subchannel with only zeros in the KCHANA or KCHANB arrays.

There are three basic patterns possible for vertical connections between subchannels:

(a) one subchannel below connected to one subchannel above;

(b) one subchannel below connected to many subchannels above; and

(c) multiple subchannels below connected to a single subchannel above

As previously discussed, the momentum equation for the axial velocity at the section boundary is solved at the top node of the bottom subchannel for case (a); in the bottom node of each of the upper subchannels in 


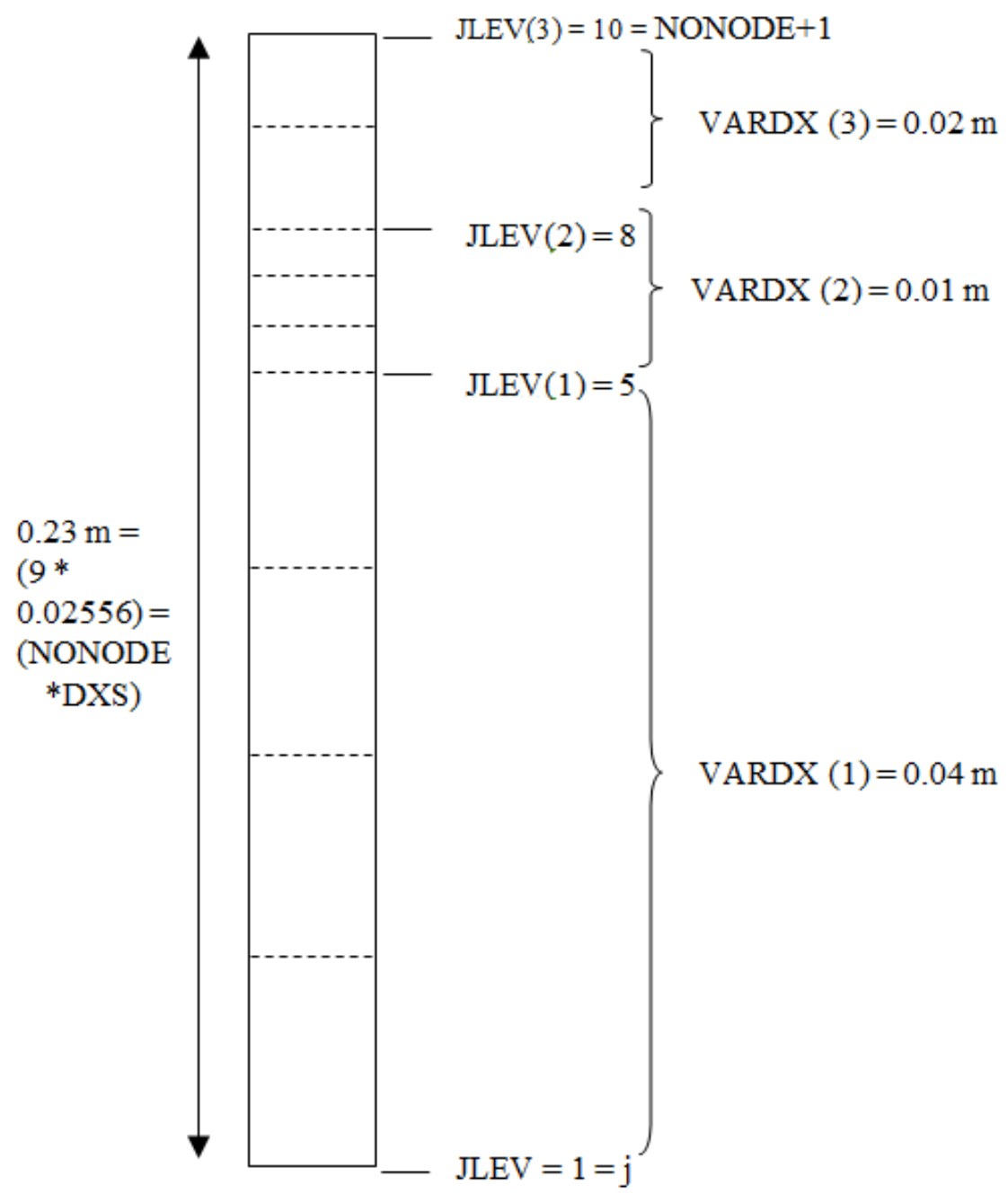

Figure 23.13: Diagram of the variable axial node length 

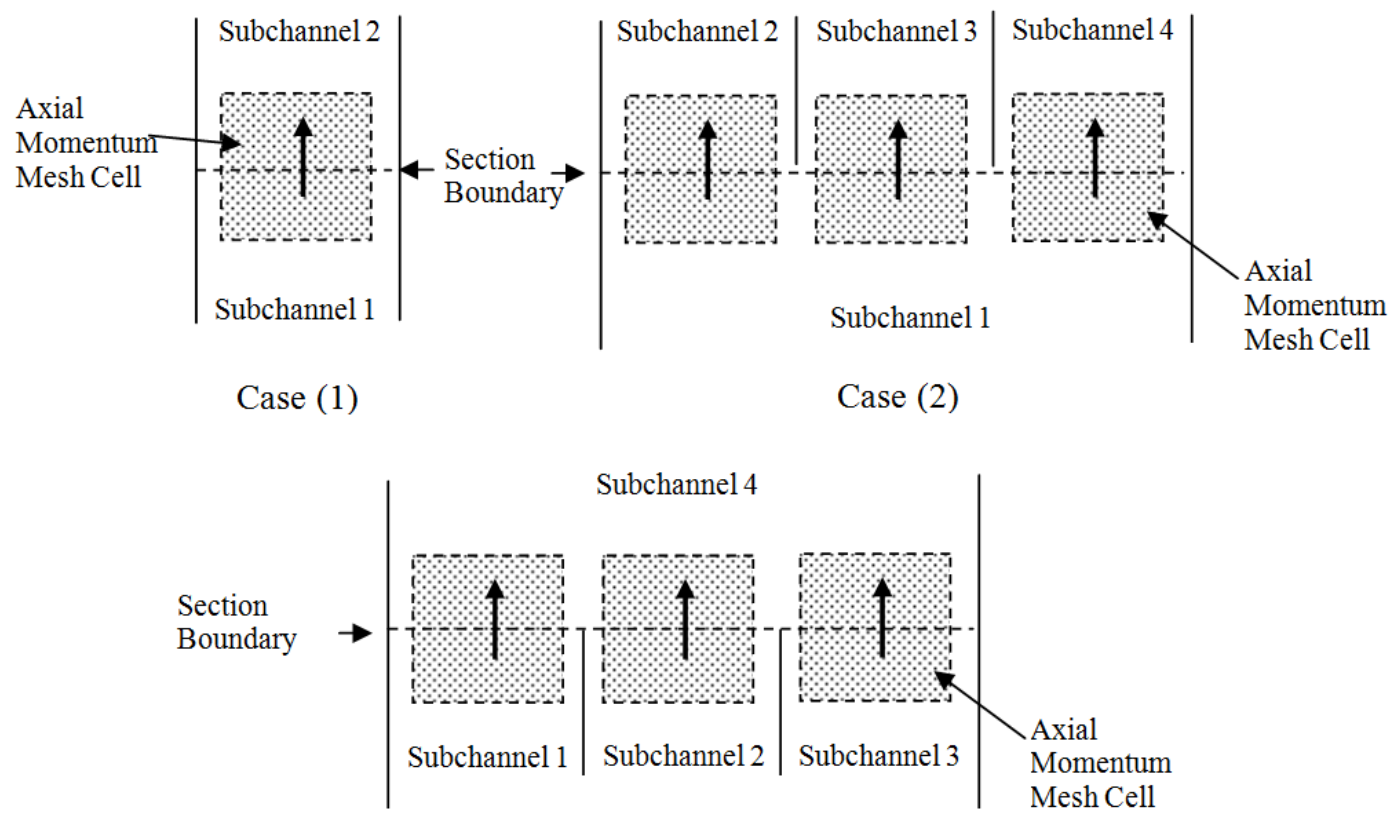

Case (3)

Figure 23.14: Allowable vertical connections between subchannels at section boundaries

case (b); and in the top node of each lower subchannel in case (c). The momentum mesh cells at the section boundary for these three cases are shown in Figure 23.14. Velocities are solved for in the momentum cells at the top of subchannel 1 for case (a); at the bottom of subchannels 2, 3, and 4 for case (b); and at the top of subchannels 1, 2, and 3 for case (c).

Velocities at the momentum cell faces, which are used to calculate the momentum flux terms, are obtained by averaging the velocities at the cell centers of adjacent momentum cells. At section boundaries where the connections are like those shown in cases (a) and (c) in Figure 23.14, the velocity at the top face of the momentum cell is obtained by averaging the velocities at the centers of the momentum mesh cells on the boundary with the velocity at the center of the first momentum mesh cell in the subchannel above. For case (a), the velocity of subchannel 2 , node 2 , is averaged with the velocity calculated in subchannel 1 at the boundary. For case (c), the velocity of subchannel 4, node 2, is averaged with the velocities calculated in subchannels 1, 2, and 3 at the boundary. For case (b), the velocity calculated at the last cell of subchannel 1 is averaged with velocities calculated in subchannels 2, 3, and 4 at the boundary. A subchannel with multiple connections to both the bottom and top must contain at least two mesh cells. If the subchannel had only one cell, the code would not be able to determine which velocities to use when obtaining average velocity at the cell face for momentum cells at the bottom of the subchannel or at the top of the subchannel.

Figure 23.15 shows two examples of subchannel connections that are not permitted by the subchannel splitting logic of the code. In example (a), the subchannels below overlap in their connections to the subchannels above. In example (b), subchannel 3 is only one cell long. The input for the correct subchannel splitting example shown in Figure 23.15 (b) will have the following format: 


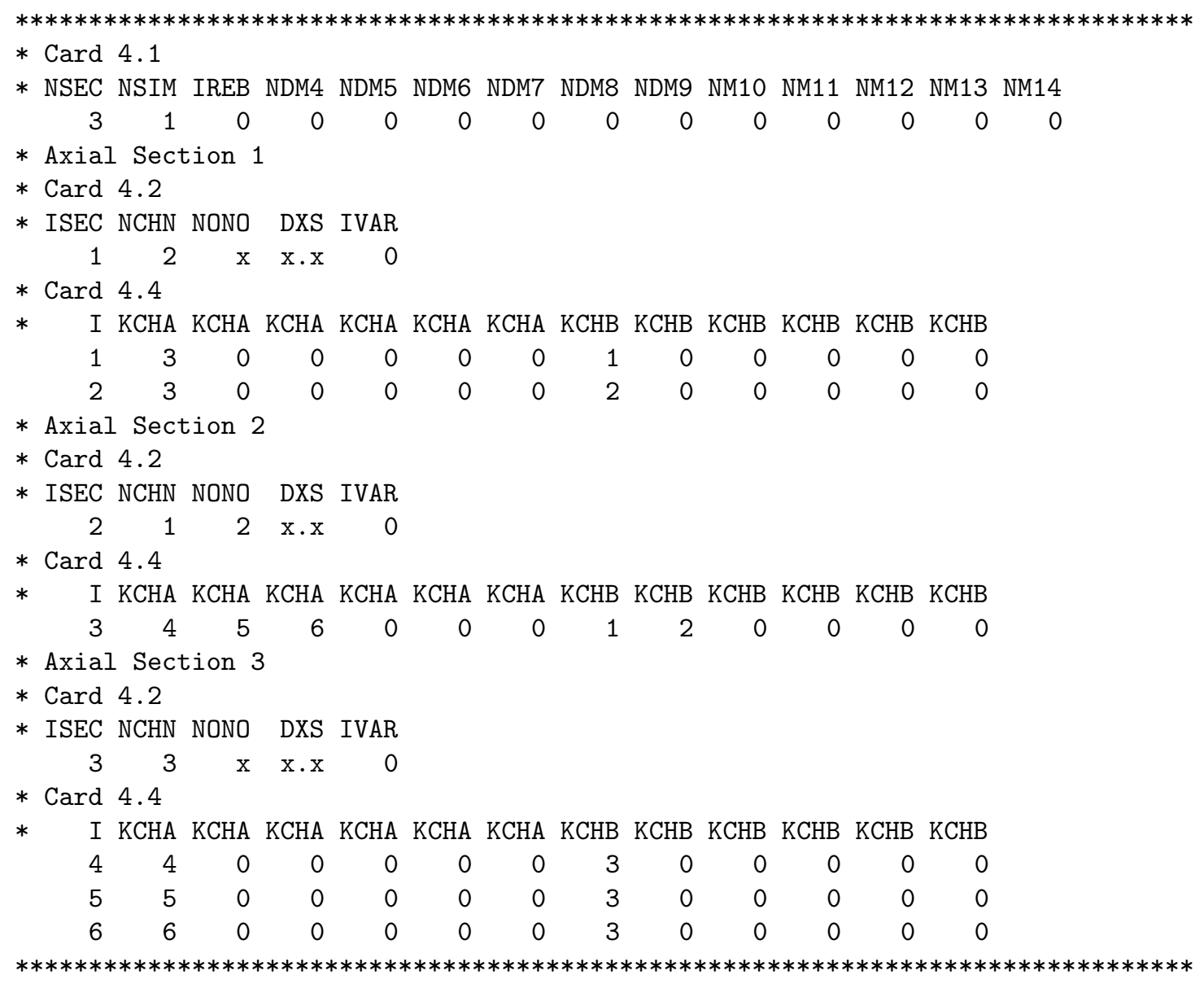

The remainder of CARD GROUP 4 specifies the numerical solution procedure to be used for solving the linearized continuity and energy equations. The equations may be solved by a direct inversion of the system pressure matrix or a combination of direct inversion and Gauss-Seidel iterative method. (The pressure matrix can be solved using different Krylov iterative solvers, but this option is controlled by the flag ISOL entered in CARD GROUP 1, ISOL > 0). In the combination solution, the pressure matrix for groups of cells is solved by direct inversion while the pressure in cells surrounding each group is held constant. Gauss-Seidel iteration is then performed over the groups of cells to obtain a converged solution for the entire system pressure matrix.

The solution procedure used is determined by the parameter NSIM read on CARD 4.1. If the number of the simultaneous solution groups NSIM is set to one the solution is obtained by direct inversion. If NSIM is set to $N$ (where $N$ is an integer greater than one) the solution is obtained with a combination of direct inversion and iteration with $N$ simultaneous solution groups. The convergence rate for the iterative solution can be enhanced by specifying the rebalancing option, IREBAL $=1$. When rebalancing is done, a one-dimensional solution for the linear pressure variation at each axial level is obtained by direct inversion. This value is used as an initial estimate for the linear pressure variation in each cell in the group-by-group iteration.

A direct inversion is recommended for relatively small problems (up to 100 mesh cells). The Gauss-Seidel 

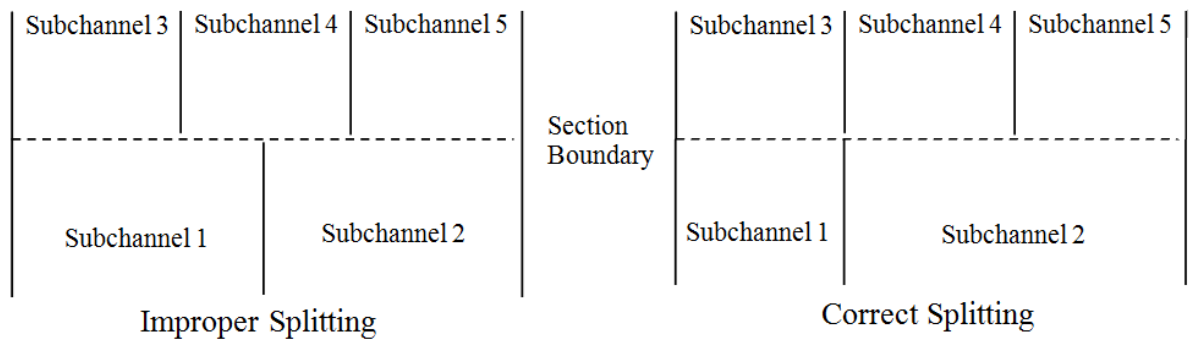

(a)

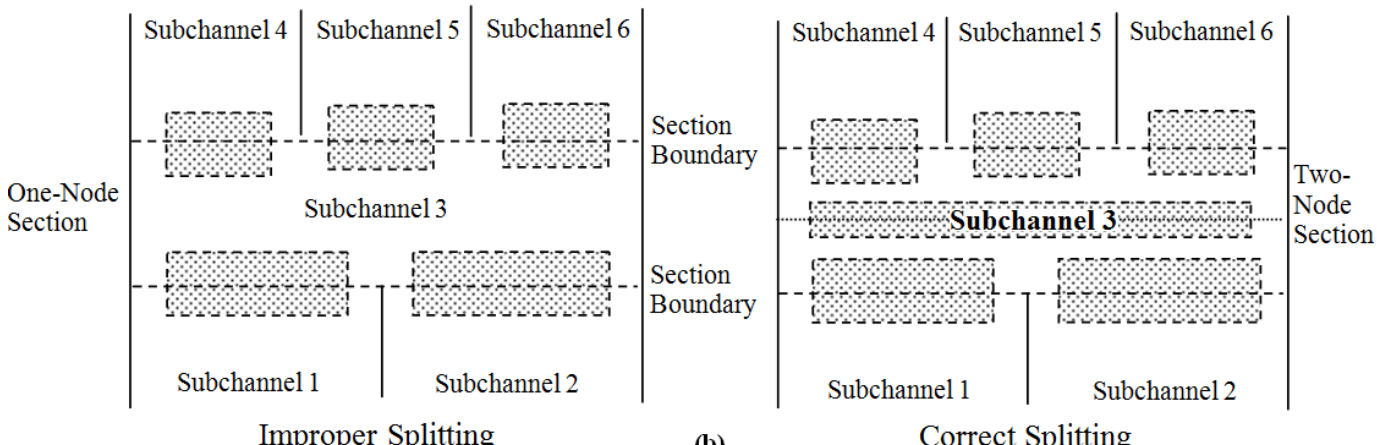

(b)

Correct Splitting

Figure 23.15: Common subchannel splitting errors

iterative technique is recommended for larger problems. However, experience indicates that the significant speed-up with group-by-group iteration is observed only when stationary conditions or transients not involving mass flow rate variation are simulated. For flow transients, the Gauss-Seidel solver converges slowly, leading to a tremendous increase of the CPU time (such lack of convergence cannot be overcome by using the rebalancing option). Therefore, for larger problems, the Krylov solvers are highly recommended because of their competitive efficiency and better accuracy comparing to the Gauss-Seidel method.

The bandwidth of the pressure matrix is defined by the maximum difference between the index numbers of adjacent cells in a group that is being solved simultaneously. This maximum difference is specified by input as parameter IWIDE on CARD 4.5, and the maximum bandwidth of the pressure matrix is calculated as $2 *$ IWIDE. The cell numbers used to define IWIDE are not subchannel numbers. Cell numbers are assigned in the following manner: beginning at the first subchannel in the first axial section, the first node in the subchannel (node $J=2$ ) is assigned cell number one (1). The cells of all the subchannels in that section are numbered sequentially at the same level $(J=2)$. The cell numbering is continued on the next level, starting in the same first subchannel. This process continues until all cells for all axial sections have been assigned unique cell numbers. An example illustrating this numbering convention is shown in Figure 23.16. In the example, IWIDE is equal to 2 in simultaneous solution groups 1 and 5 . In groups 3 and 4, IWIDE $=1$. In group 2 IWIDE is 3 . The value of IWIDE entered in CARD 4.5 will then be 3 (the matrix must accommodate the largest bandwidth in all groups).

The MSIM array - the number of the last cell in each simultaneous solution group - is provided by CARD 4.6. The input for the example shown in Figure 23.16 will have the following format: 


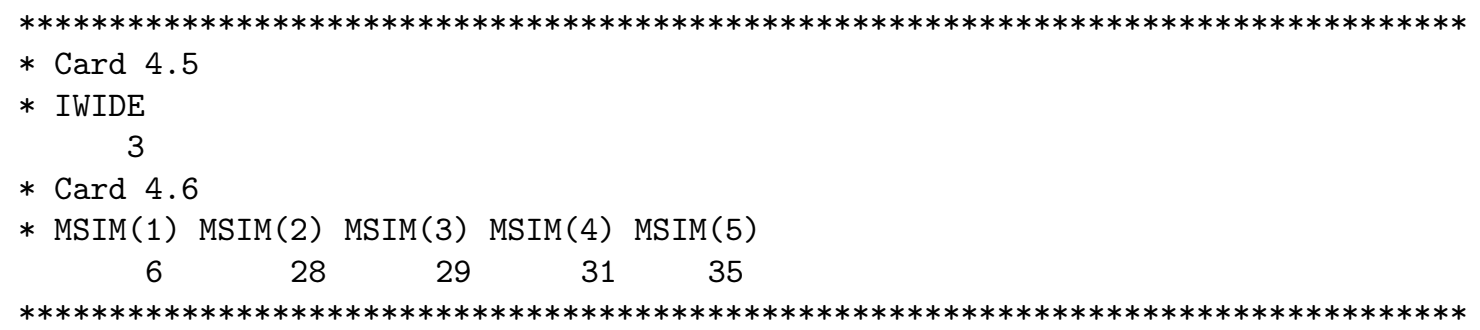

\subsubsection{Instructions to CARD GROUP 5 and 6}

The geometry of a subchannel or a gap is assumed to remain constant along the entire axial length at the nominal values specified by input. But CARD GROUPS 5 and 6 permits the user to specify axial variation in the geometry. This is an optional input and it is omitted if axial variation data is not needed. The input data is extremely general. The user provides tables of variation factors in CARD GROUP 5. Variation tables can be supplied for the continuity flow area, momentum flow area, and wetted perimeter of subchannels and the width of gaps. Variation tables are read on CARD 5.3 as tables of node number, JAXL ( I,N), versus variation factor $\operatorname{AFACT}(I, N)$. The node number JAXL $(I, N)$ refers to the continuity cell for continuity area and gap width and to the momentum cell for the momentum area and wetted perimeter. The variation factor is defined as:

$\operatorname{AFACT}(\mathrm{I}, \mathrm{L})=\frac{\text { localvalue }}{\text { nominalvalue }}$

The value $\operatorname{AFACT}(I, N)$ is applied in the code as a multiplier on the nominal value of the quantity being varied at the corresponding JAXL (I,N) node. Figure 23.17 shows two examples of subchannels with area variations that can be modeled with variation tables. For example (a), variations of the continuity area occur at nodes 7, 8, and 9. Variations in the momentum area also occur at nodes 7, 8, and 9, but because the geometry of the subchannel is tapered and the continuity and momentum nodes are staggered, the areas vary by different amounts. The tapered region in the subchannel is approximated by a stack of cells that have a uniform cross section along their individual lengths. The continuity areas of the cells should be defined so that the volumes of the cells are equal to the volumes of the regions they are intended to model. In the continuity solution, cells 7 and 8 model the tapered region. The momentum cells use the actual area at the location of the momentum cell center. In this example, the momentum area variation is modeled in cell 7. The wetted perimeter is defined in the momentum cell, so variations in wetted perimeter must be located relative to the momentum cells. In example (a) of Figure 23.17, the gradually changing wetted perimeter must be approximated by step changes, as is the gradually changing flow area in the taper. For the circular cross section of this example, the simplest approach is to define the wetted perimeter as the perimeter of the momentum cells:

$P_{w}=\pi D^{\prime}$

where:

$D^{\prime}=\sqrt{\frac{4 A_{\text {momcell }}}{\pi}}$

The input for CARD GROUP 5 to model the variations in example (a) of Figure 23.17 consists of three variation tables $($ NAFACT $=3$, read on CARD 5.1). The tables themselves are read on CARD 5.2 (specifying 


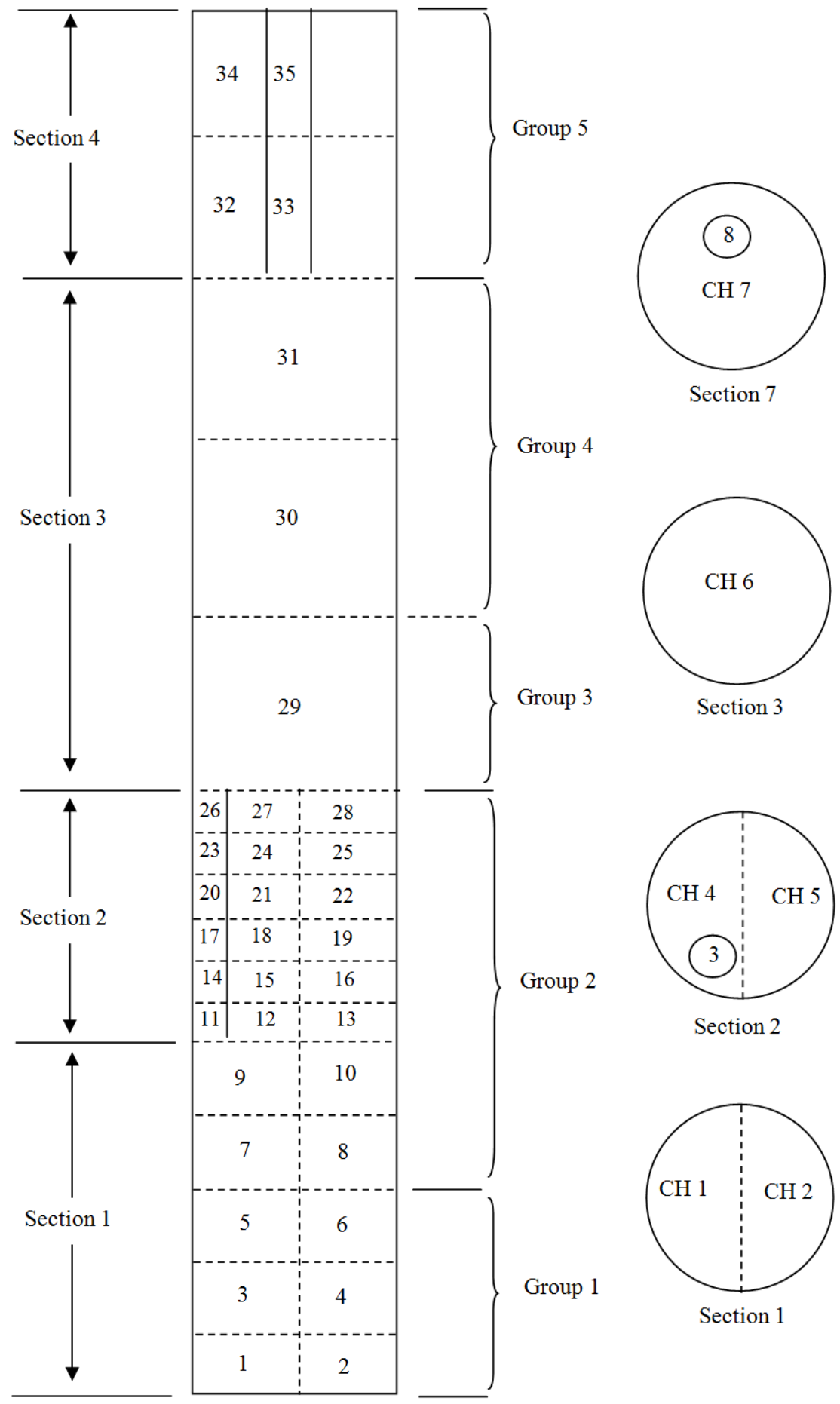

Figure 23.16: Common subchannel splitting errors 
the number of entrees in a table) and on CARD 5.3 (filling the arrays for the node indices, JAXL, and variation factors, AFACT). The tables are numbered sequentially in the order in which they are read.

Example (b) in Figure 23.17 shows a different sort of area variation. The orifice plate in the straight pipe affects the momentum solution, but because it occurs over a relatively short distance compared to the node length, the continuity solution is largely unaffected. This could be modeled with a momentum area variation alone without any variation in the continuity cell area.

Gap width variations are specified in the same manner as axial variations in the wetted perimeter. The nodes that differ from nominal are identified in the JAXL array and the corresponding variation factors in the AFACT array.

The input for the example shown in Figure 23.17 (a) will have the following format:

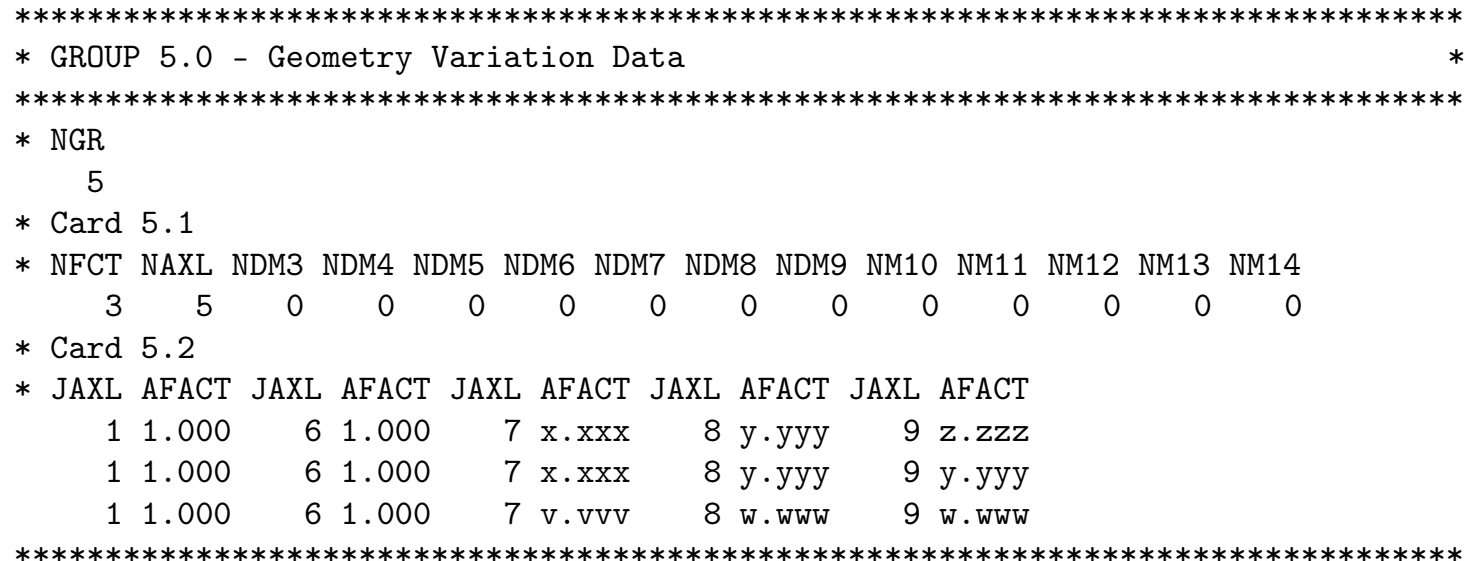

Variations encountered in most problems for CTF will not be as neat, generally, as the two examples shown in Figure 23.17. The user will have to make approximations appropriate for the particular geometry involved and noding selected. Some general guidelines in setting up axial variations are:

1. In complex geometries, the user should preserve the same fluid volume as in the actual system.

2. The momentum area should approximate the actual flow area in the system at sudden changes.

3. The code interpolates linearly in the table to obtain variation factors for cells within the range of the table but not named explicitly. (Cells with identification numbers lower than the first element of a variation table or greater than the last element in the table remain at nominal values).

4. The area in either the continuity cells or the momentum cells should not change by more than a factor of two between adjacent cells, even if the area in the system actually changes more abruptly. The code must be led gradually through a large change in a series of steps.

The input for CARD GROUP 6 specifies the subchannels or gaps to which the variation tables described in CARD GROUP 5 apply. Variation table assignments are read on CARD 6.2 for either subchannels or gaps. 


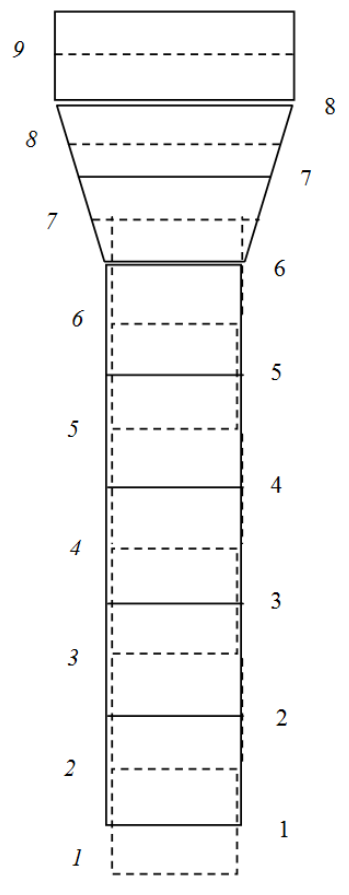

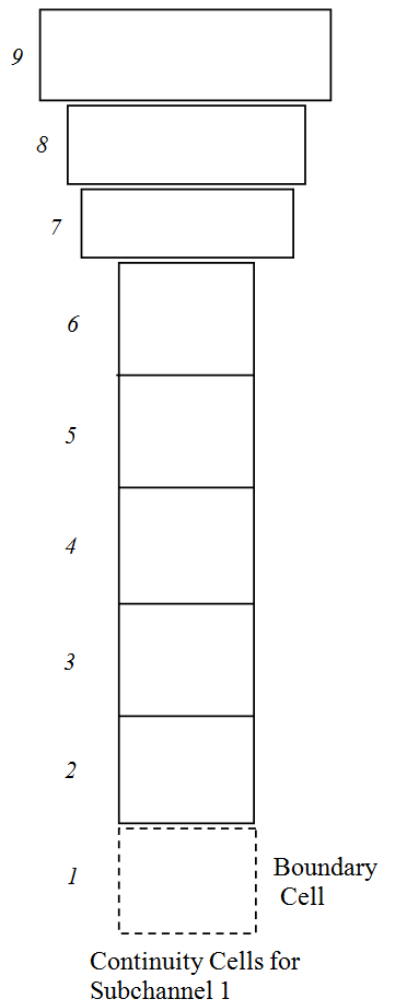

(a)

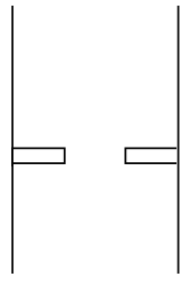

(b)

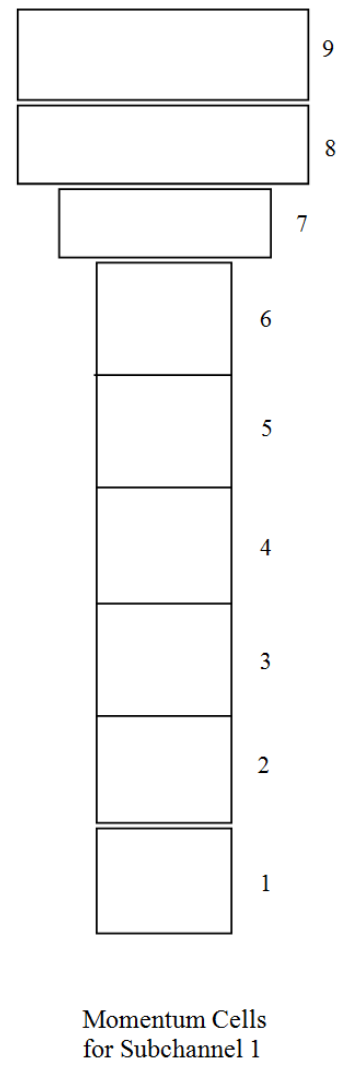

Figure 23.17: Examples of axial variation in continuity and momentum area and wetted perimeter of a subchannel 
The variation tables are identified by sequence number; i.e., the first table read in CARD GROUP 5 is Table 1; the second table read in CARD GROUP 5 is Table 2; etc. The index number of the table to be used for continuity area variations is specified by IACT. The index number of the table to be used for momentum area variations is specified by IAMT. The index number of the table to be used for wetted perimeter variations is specified by IPWT. The numbers of the subchannels using the variation tables named by IACT, IAMT, and IPWT are listed in array ICRG(M). Axial variation in gap width is specified by setting IACT to the negative of the variation table number, and naming the indices of the gaps using the table in array ICRG (M). When gap variations are specified, IAMT and IPWT are not used.

The input for CARD 6.2 is repeated until all subchannels and gaps having variations have been identified. It is not necessary to specify variations in continuity area, momentum area and wetted perimeter in a subchannel simultaneously. Any or all can be used, as is appropriate for a given problem. It is possible to specify only a continuity variation, only a momentum area variation, only a wetted perimeter, or any combination of the three.

The input for the example shown in Figure 23.17 (a) will have the following format:

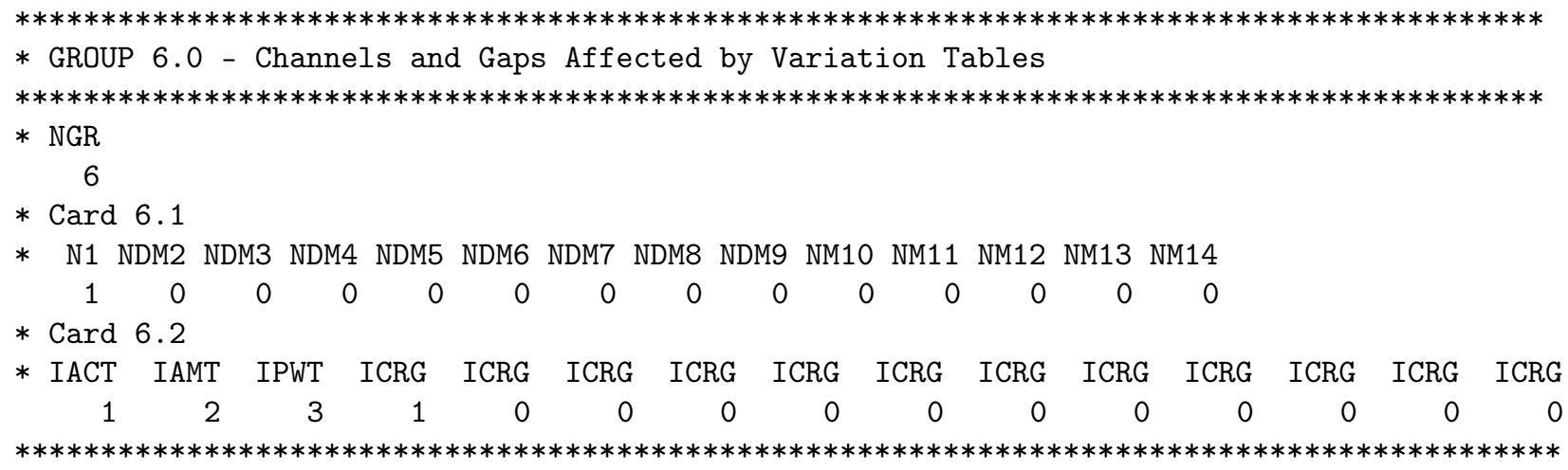

\subsubsection{Instructions to CARD GROUP 7}

The input for CARD GROUP 7 is designed to model local pressure losses in the axial flow due to spacer grids, orifice plates and other local obstructions in the flow field.

In the COBRA/TRAC code version [3], the local pressure losses in vertical flow are modeled as velocity head loss given by

$$
\Delta P=\zeta \rho \frac{v^{2}}{2 g_{c}},
$$

where $\zeta$ is the pressure loss coefficient, $\rho$ is the density, $v$ is the flow velocity in vertical direction, and $g_{c}$ is the gravitational conversion constant. The pressure loss coefficients are defined assuming positive upflow in a channel and specified for a momentum (not continuity) cell containing the obstruction.

Later, grid heat transfer models for convective enhancement downstream of the spacers, grid rewet during bottom reflood, and droplet breakup on spacers were included in Paik et al. [4]. In this code version, rather 
than using input specified values for the spacer loss coefficients, the spacer loss coefficients are calculated from grid dimensions using

$$
\zeta_{\text {grid }}=\min \left(20,196 R e_{\text {mix }}^{-0.333}\right) f_{\text {loss }}\left(A_{\text {blocked }}^{\text {spacer }}+A_{\text {blocked }}^{\text {springs }}\right)^{2},
$$

where

$f_{\text {loss }}$ is the pressure loss coefficient multiplier (input value),

$A_{\text {blocked }}^{\text {spacer }}$ is the fraction of channel area blocked by the grid (input value),

$A_{\text {blocked }}^{\text {springs }}$ is the fraction of channel area blocked by the grid springs (input value), and

$R e_{m i x}$ is the drops/bubbles mixture Reynolds number.

In the current code version, pressure losses must be specified either by $\zeta$ values or by geometrically modeled flow blockages. If pressure loss coefficients, CDLs, are specified in CARD 7.2, then cards CARD 7.3 to CARD 7.9 must be omitted.

CARD 7.1 specifies the total number of loss coefficients, NCD; the number of grid types, NGT; the flag for grid quench front model IFGQF; the flag for small droplets model, IFSDRP; the flag for grid convective enhancement, IFESPV; the flag for two-phase enhancement of dispersed flow heat transfer, IFTPE; the flag for grid quench calculations, IGTEMP; the flag for flow blockages calculations NFBS; and IXFLOW, the flag for grid spacer influences on lateral exchange over gaps.

If the parameter NCD is equal to $\mathrm{N}$, loss coefficient values $\mathrm{CDL}(\mathrm{N})$ are specified on CARD 7.2. CARD 7.2 contains also the identification number $J$ of the axial node at which the pressure loss coefficient is applied and the ICDUM array of identification numbers of the subchannels for which the pressure loss coefficient is applied at axial node $J$. The pressure losses will be calculated according to Equation 23.1. The models for quench front and grid convective enhancement are not applicable when NGT $=0$ (NCD >0) and the flags IFGQF and IFESPV have to be set to zero in CARD 7.1. The input for the configuration shown in Figure 23.18 will have the following format:

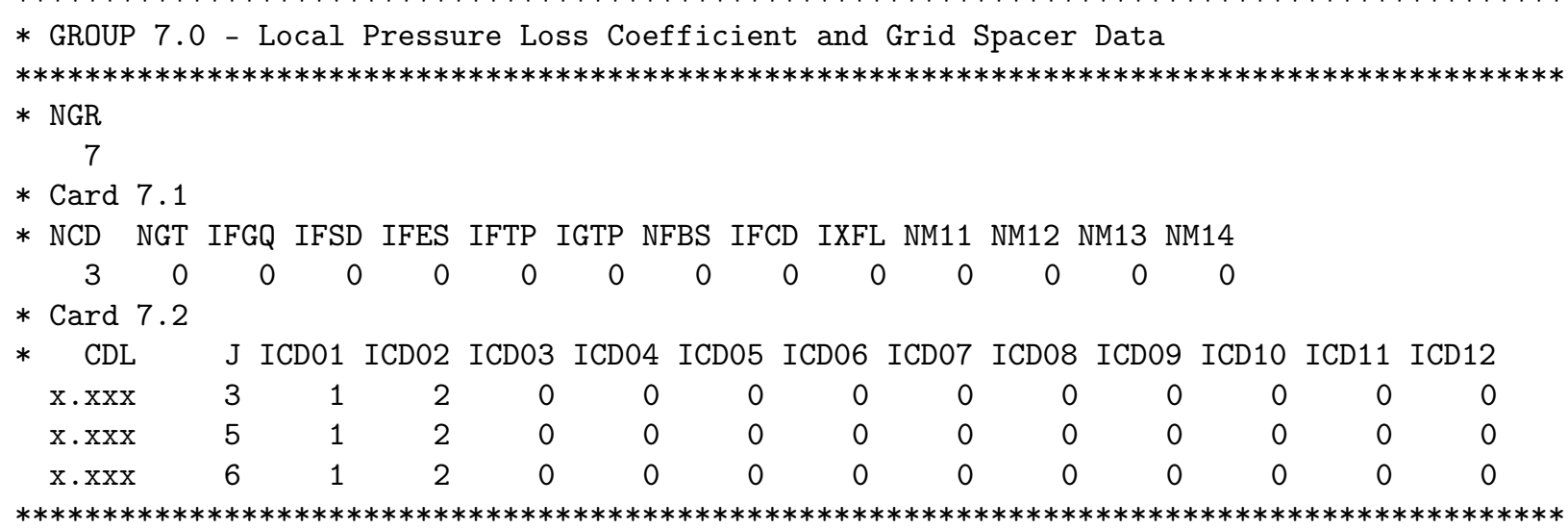

There are two important points to remember: 


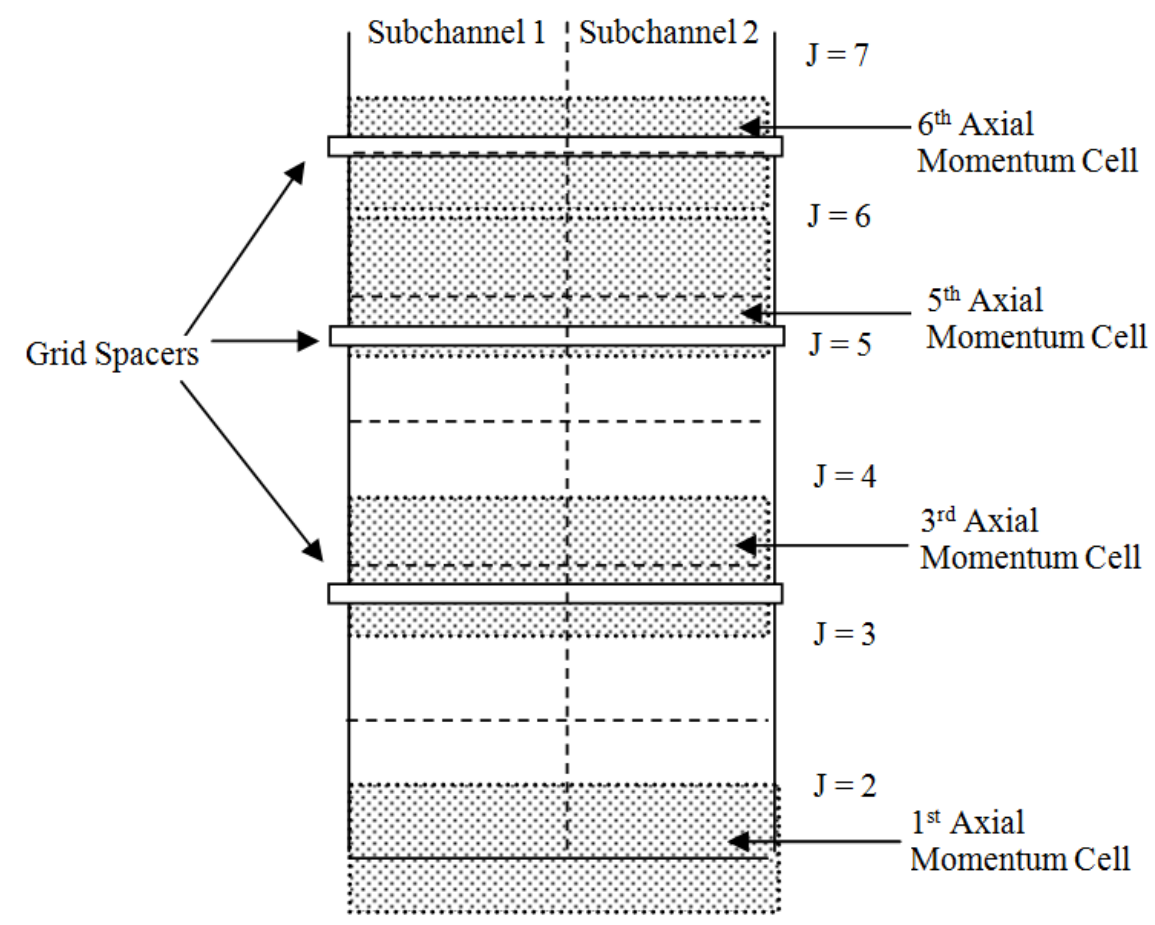

Figure 23.18: Example of subchannels with local form losses due to spacer grids

1. The location of a loss coefficient is determined by node and subchannel number. The node refers to the momentum cell, not the continuity cell. This must be kept in mind when determining the node that corresponds to the location of the local loss in the system being modeled. Care must be used when placing a loss coefficient in a momentum cell at a section boundary. The loss coefficients must be defined in the subchannel where the momentum equation is solved.

2. The loss coefficients are defined assuming positive upflow in the subchannel. If the loss coefficient of a particular structure changes significantly when flow reverses through it, the code does not see the change. If reverse flow is the dominating pattern for such a situation, the user should specify the loss coefficient corresponding to reverse flow than the value for positive flow.

If the parameter NGT is greater than zero (0), the pressure loss coefficients will be calculated according to the methodology described in Paik et al. [4]. This option requires input for CARDS 7.3, 7.4, and 7.5. The physical meaning of the parameters that have to be specified is given in the input description for CARD 7 . An example of the input for the arrays NGROD and NGSURF is given below. It is a four subchannels problem with three spacer grids of same type ING along the axial length. The axial locations of the grids, NNGL, refer to the momentum cells containing the grids. 

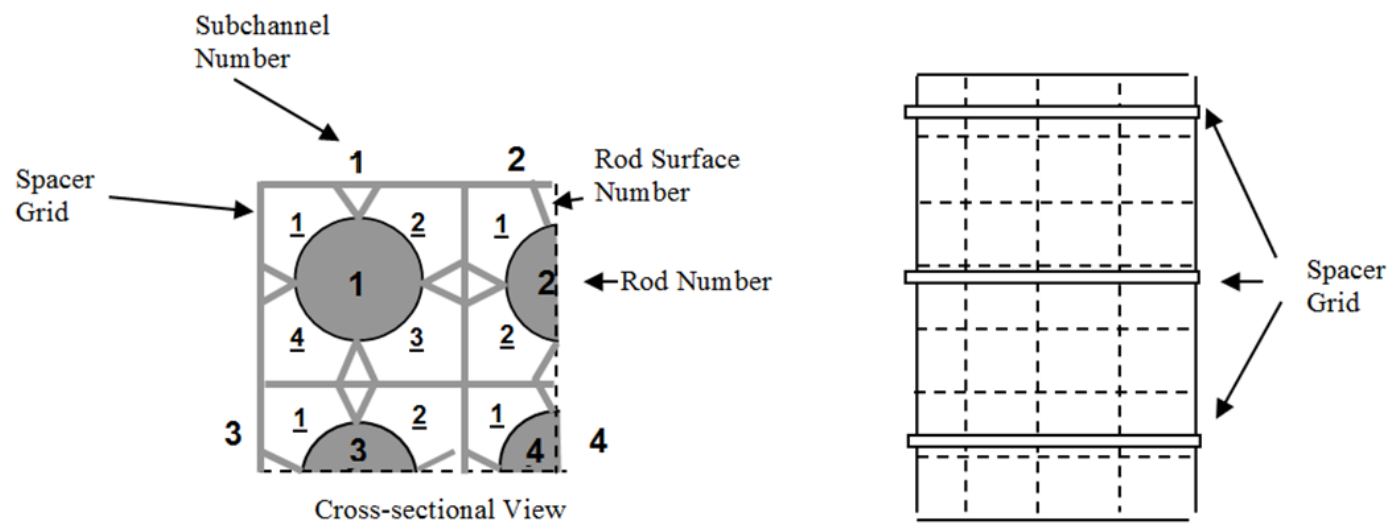

Figure 23.19: Example of rod surface numbering in CARD 7.5

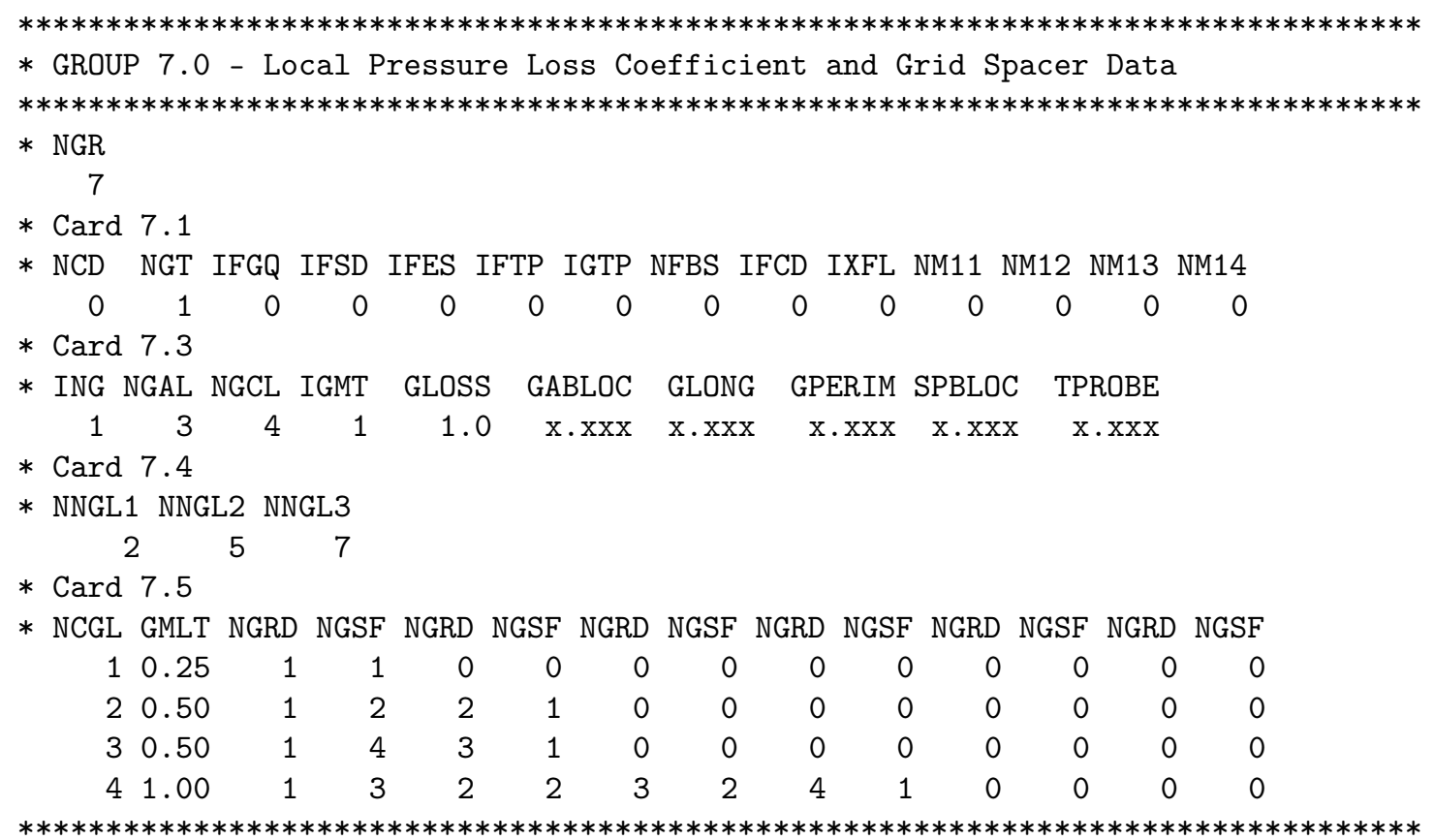

CARDS 7.6 and 7.7 are needed when flow blockages are modeled. CARD 7.8 provides input data for the grid quench calculations. For a detailed description of the flow blockages modeling, the user should refer to Paik et al. [4].

CARD 7.8 is used if the updated spacer grid quench modeling is desired, which uses the grid re-wet model to calculate grid temperatures.

CARD 7.9 is used if the spacer grid crossflow effects are modeled. When IXFLOW $\neq 0$ then this card is required. The user must be able to supply data files on the spacer grids depending on the modeling option chosen. If IXFLOW $=1$, then the directed crossflow modeling option is activated. If this model is invoked, 
then files 'xflow_data' and 'dirct_data.inp' must be provided in the working directory.

The first file, 'xflow_data', must contain a 2D table for the lateral flow rates corresponding to the spacer grid in use. This $2 \mathrm{D}$ table should contain vane angles (degrees) in the $\mathrm{x}$-direction and axial distance $(\mathrm{m})$ from the bottom of the rod in the y-direction.

The first non-commented line for the file must contain 4 values:

1. the number of axial distances at which data are specified;

2. the number of angles for which lateral flow data are provided;

3. dummy variable (suggested value of 0 ); and

4. dummy variable (suggested value of 0 )

The second non-commented line for the file must contain 1 value: the number of data sets (2D tables) to be read.

The third non-commented line for the file must contain N2 values (as specified by the number of mixing vane angles in the first line): the angles at which data are supplied.

The remaining lines in the document should contain N2 +1 values each. The first column corresponds to the axial position $(\mathrm{m})$ and the remaining columns are the spacer multiplier values corresponding to the current axial location and angle.

A sample of this file is provided below. This sample provides data for 2 different angles ( 0.0 and 15.0 degrees) at 9 different axial locations from $0.0000 \mathrm{~m}$ to $2.0000 \mathrm{~m}$. The ' 0.0000 ' values would be replaced by the values of the spacer multiplier at the given angle.

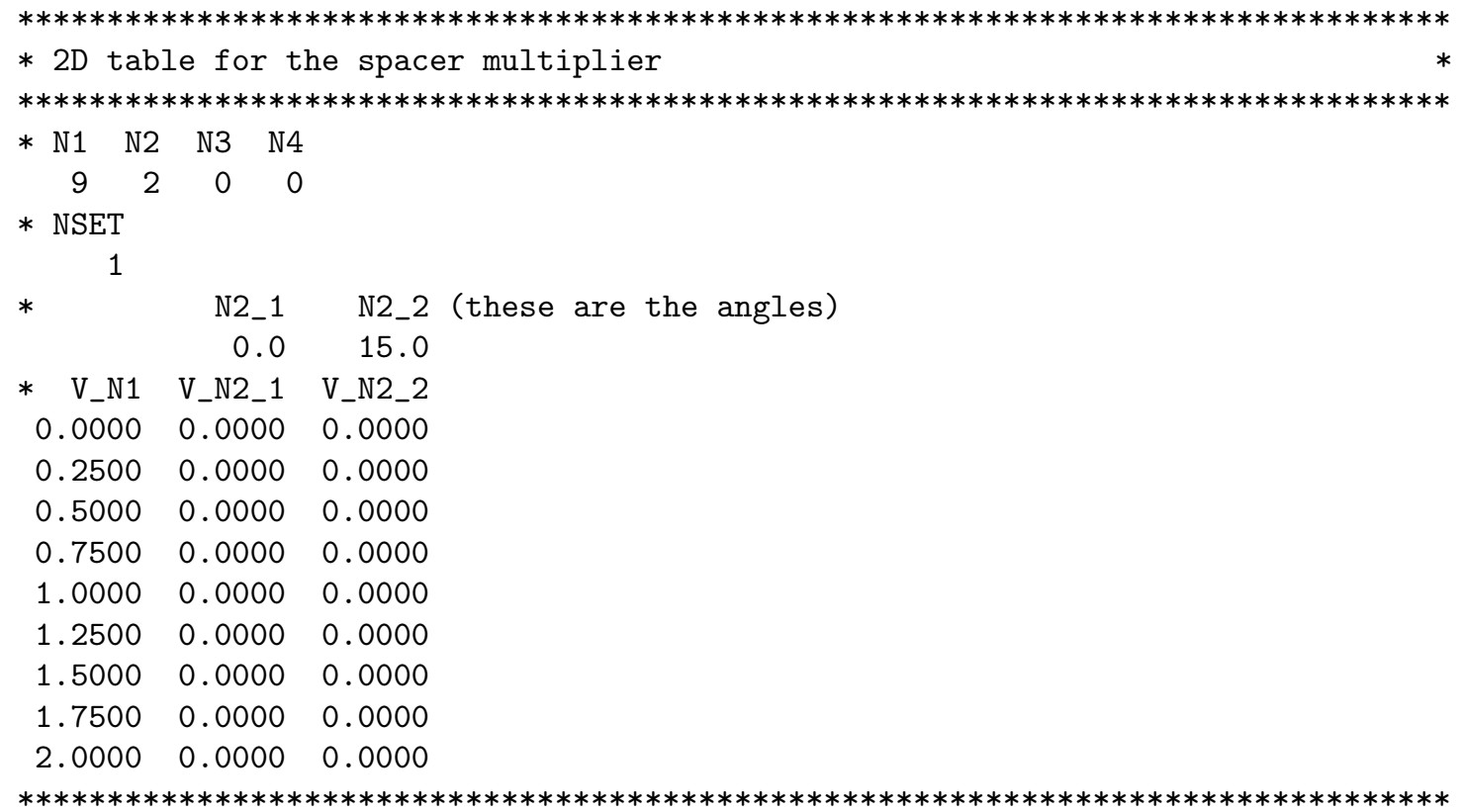


The data for these files are generally based on CFD calculations and are specific to each spacer grid design. The code linearly interpolates between the angles at which the data are specified to match that of VAL_ANG in the input deck, CARD 7.9.

\subsection{Specification of the Conductors' Data}

This section provides the user with guidance how to set up the input data required for the solution of the heat transfer and heat conduction. It covers the input for the heated and unheated conductors and specification of their material properties.

\subsubsection{Instructions to CARD GROUP 8}

The input for CARD GROUP 8 identifies the rods and unheated conductors modeling the solid structures that interact significantly with the fluid in a particular problem.

Rods and unheated conductors are both used to model solid structures in the vessel. There are two significant differences between them. First, rods can model either active or passive elements, but unheated conductors are always passive. Unheated conductors cannot have internal heat sources. Second, the quench front model with fine-mesh re-noding can be applied to rods if needed, but unheated conductors are assumed to never require it.

Examples of vessel structure that can be modeled with rods are:

1. an array of nuclear fuel pins;

2. an array of electrically-heated fuel pin simulators;

3. an electrically-heated annular test section with both cylinders heated;

4. a test section with an electrically heated flat plate as the heat source; etc.

Examples of vessel structure that can be modeled with unheated conductors are:

1. a control rod guide tube;

2. a support column;

3. a section of downcomer annulus;

4. a canister of a BWR fuel assembly; etc.

CARD 8.1 identifies the number of rods in the problem, NROD; the number of unheated conductors, NSROD; the conduction solution flag, NC; the number of temperature initialization tables, NRRAB; the number of radiation channels, NRAD; the flag for steady-state calculation of rod temperature, NSTATE; the number of time steps 
between radiation calculations, NXF; the flag for Yamanouchi canister quench model, NCAN; the flag for the radiation heat transfer calculations, RADFLG; and the flag for critical heat flux calculation option.

The conduction solution flag, NC, must be set to select among no conduction (NC $=0$ ); radial conduction only $(\mathrm{NC}=1)$; radial and axial conduction ( $\mathrm{NC}=2)$; and radial, axial, and azimuthal conduction ( $\mathrm{NC}=3$ ). The option for radial and axial conduction is recommended only for problems where a significant axial temperature gradient in the rods is expected. Similarly, if significant axial and azimuthal temperature gradients are expected, the NC parameter is set to three (3). Problems involving reflooding and quenching of very hot surfaces should not use the radial conduction option only. Problems involving relatively gradual heating or cooling of the system can be handled adequately with radial conduction only.

The simulation time can be speed-up by selecting the option for a steady state fuel rod temperature calculation (NSTATE is set greater than zero).

The rod identification parameters are read on CARDS 8.2, 8.3, and 8.4. If NROD is set to zero these cards are omitted. The rods are numbered sequentially from 1 to NROD. Each rod is uniquely identified by its index number, $N$, and geometry type number IFTYP $(N)$. The geometry type number corresponds to a set of descriptive geometry data specified in CARD GROUP 9. An individual rod may have a unique geometry type, or several rods may be of the same geometry type.

Each rod has identified with it the number of an axial power profile table, IAXP(N). If IAXP(N) is left blank, an axially uniform table with a factor of unity is assumed. An axial profile table can serve any number of rods. The axial power profile tables (and the radial power factors) are specified in CARD GROUP 11. Together, the axial profile tables and the radial power factors define the local power generation in the individual rod.

In modeling large geometries it is sometimes convenient to represent regions of the vessel by average rods. A fuel pin array might be represented by a single average rod. The number of actual rods modeled by an average rod is specified in the variable RMULT $(\mathrm{N})$. The values specified for RMULT can include fractional parts of rods.

The fine-mesh re-noding capability developed to resolve the quench front in reflooding requires some extra input for the rods. If fine-mesh re-noding is to be used for a particular rod, the flag $\operatorname{NRENODE}(\mathrm{N})$ is set to the number of calculational time steps to elapse between re-noding. How often the rod should be re-noded is primarily a function of the reflood rate and the size of time step expected during the reflood portion of the transient. In general, NRENODE (N) should be set that the quench front will not progress further than $1 / 2$ of the minimum node size, DAXMIN $(\mathrm{N})$, between re-noding. For example, if the quench front velocity is 0.5 $\mathrm{cm} / \mathrm{sec}$, the maximum time step is 0.05 seconds, and the minimum node size is $0.1 \mathrm{~cm}$, then:

$\operatorname{NRENODE}=\operatorname{INT}\left(\frac{1 / 2 *(0.1)}{(0.5)(0.05)}\right)=2$

If the rod is a tube quenching on the inside surface, then NRENODE $(N)$ should be specified as a negative number and the absolute value of $\operatorname{NRENODE}(\mathrm{N})$ is used to determine the re-noding interval.

Variable $\operatorname{HGAP}(\mathrm{N})$, entered in CARD 8.2, specifies a constant value for the fuel rod gap conductance. (If a dynamic gap conductance modeling is desired, the required input will be provided by CARD GROUP 9). If the rod $N$ does not model a nuclear fuel rod, $\operatorname{HGAP}(\mathrm{N})$ should be set to zero.

The code allows a rod to be included in more than one axial section. The total number of sections containing $\operatorname{rod} N$ is specified by the parameter ISECR $(\mathrm{N})$. 
The last two variables in CARD 8.2, $\operatorname{HTAMB}(\mathrm{N})$ and $\operatorname{TAMB}(\mathrm{N})$, provide input data for the heat transfer coefficient for heat loss to ambient from a surface not connected to a subchannel and the sink temperature for ambient heat loss. This option is normally not related to the real fuel rod simulation. It is used in some simulations of experiments when the user wants to account for the heat loss from a non-water-cooled rod to the ambience or to the medium inside the tube.

CARD 8.3 describes the thermal connections between rods and subchannels for heat transfer between fluid and solid surfaces. All rods (and unheated conductors) must be connected to at least one subchannel. (However, not all subchannels have to be connected to a rod or unheated conductor). Parameter NSCHC (IS, K) gives the subchannel number with thermal connection to rod $N$ and parameter PIE(N,K) supplies the azimuthal fraction of $\operatorname{rod} N$ thermally connected to subchannel NSCHC (IS, K). Index $I S$ varies from 1 to the total number of sections containing $\operatorname{rod} N, \operatorname{ISECR}(\mathrm{N})$. Index $K$ varies from 1 to 8 (up to 8 sets of (NSCHC, PIE) may be entered. If the $\operatorname{rod} N$ is thermally connected to fewer than 8 channels, enter $(0 ; 0.0)$ until 8 sets of (NSCHC, PIE) have been entered).

If an inside surface of rod $N$ exists, the number of the subchannel connected to the connected to the inside of azimuthal section $\mathrm{K}$ of $\operatorname{rod} N, \operatorname{NISCHC}(\mathrm{N}, \mathrm{IS}, \mathrm{K})$ is provided by CARD 8.4.

The unheated conductors' identification parameters are read on CARD 8.5. If NSROD on CARD 8.1 is set to zero, CARD 8.5 is omitted. The unheated conductors are numbered sequentially from 1 to NSROD. Each unheated conductor is uniquely identified by its index number, $N$, and geometry type number ISTYP(N). The geometry type number corresponds to a set of descriptive geometry data specified in CARD GROUP 9. An individual unheated conductor may have a unique geometry type, or several unheated conductors may be of the same geometry type.

The number of actual unheated conductors modeled by a single one unheated conductor is specified in the RMULS (N) variable. The values specified for RMULS can include fractional parts of rods.

Unheated conductors do not generate heat but can transfer heat to and from the fluid and store thermal energy during a transient. For each unheated conductor $N$ the user must specify the heated perimeter of the surface, HPERIM(N). If the conductor is a tube or wall that has contact with the fluid on its inner surface, then the heated perimeter of the inner surface, HPERIMI(N), must be specified as well. The parameter NOSLCH corresponds to the identification number of the subchannel adjacent to the outside surface of the unheated conductor $N$. Respectively, the parameter NSLCHC corresponds to the identification number of the subchannel adjacent to the inside surface of the unheated conductor $N$. If no subchannels are connected to the outside/inside surfaces, NOSLCH/NSLCHC are set to zero.

Similar to the rod modeling, the last two variables in CARD 8.6, HTAMBS(N) and TAMBS(N), provide input data for the heat transfer coefficient for heat loss to ambient from surface not connected to a subchannel and the sink temperature for ambient heat loss.

CARDS 8.6 through 8.9 set up the initial surface temperate of the rods and unheated conductors. On CARD 8.9, the temperatures are specified in tables of initial temperatures, TRINIT(I,L), versus axial distance, $\operatorname{AXIALT}(I, L)$, relative to the bottom of the vessel. The code interpolates linearly in the table along the axial length of the rod or unheated conductor to which it is applied, so the first element of the AXIALT (I,L) array for a given table I must be at or below the bottom of the rod, and the last element must be at or below the top of the rod. A total of NRTAB (as specified on CARD 8.1) tables must be supplied, but table $I$ can be applied to more than one rod or unheated conductor. On CARD 8.6 the user must specify the number of rods, NRT1(I), and the number of unheated conductors, NST1(I), that use table $I$. The user 


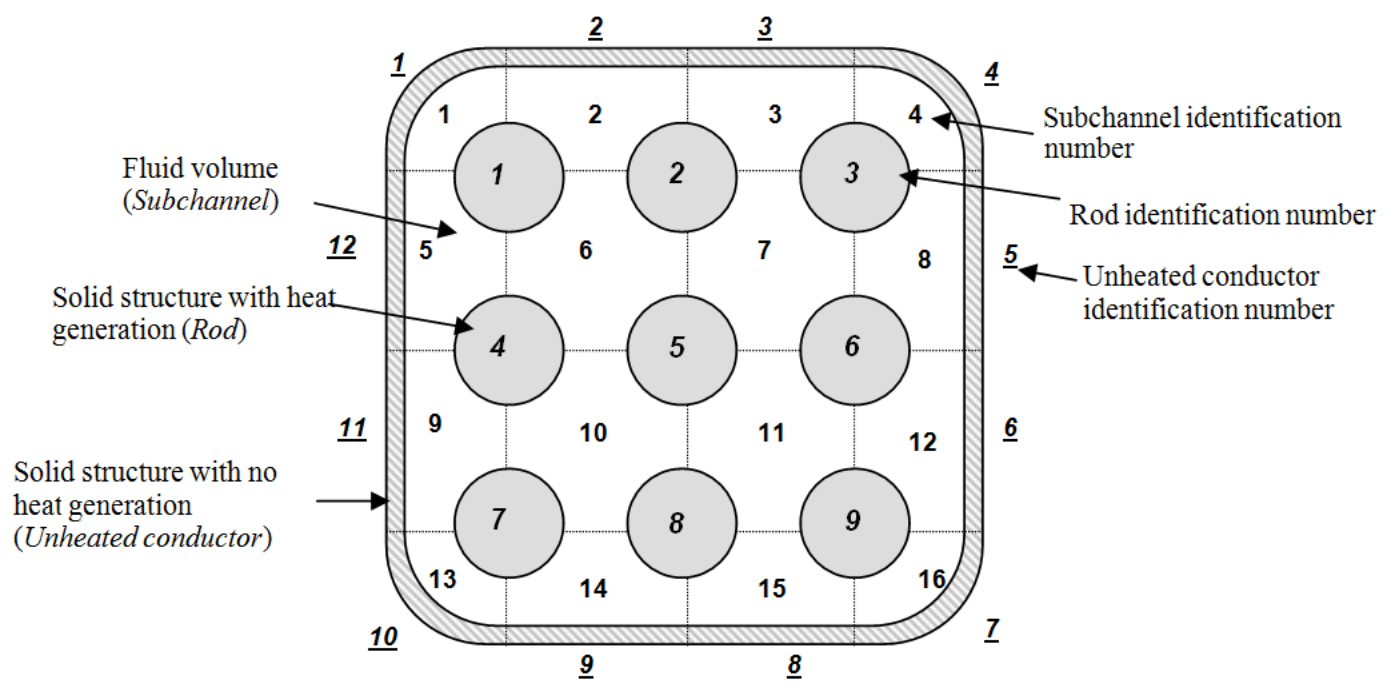

Figure 23.20: $3 \times 3$ BWR Bundle

must also identify how many pairs of $\operatorname{TRINT}(I, L)$ and $\operatorname{AXIAL}(I, L)$ elements make up this particular table. The NRAX1(I) pairs of entrees for table $I$ are read on CARD 8.9.

If table $I$ is used to initialize any rods, CARD 8.7 is read to fill array IRTAB(I,L) with the identification numbers of the rods. The steady-state conduction equation is solved for these rods using the temperatures from table $I$ as a boundary condition on the rod surfaces. If any of the rods are tubes or walls and the table is to be used to initialize the temperatures on the inside surface, the rod identification number, $N$, is entered in the IRTAB array as its negative.

If table $I$ is used to initialize any unheated conductors, CARD 8.8 is read to fill array $\operatorname{ISTAB}(I, \mathrm{~L})$ with the identification numbers of the unheated conductors. The code assumes an initially flat temperature profile in unheated conductors, so it is not necessary to specify whether the temperature applies to the inner or outer surface.

CARDS 8.10 and 8.11 are needed when radiation heat transfer must be modeled. For a detailed description, the user should refer to [4].

CARD 8.12 provides input data for the Yamanouchi canister quench model (see the CTF Theory Manual [1].

An example of CARD GROUP 8 for a 3x3 BWR bundle follows. The geometry of the bundle is shown in Figure 23.20. 


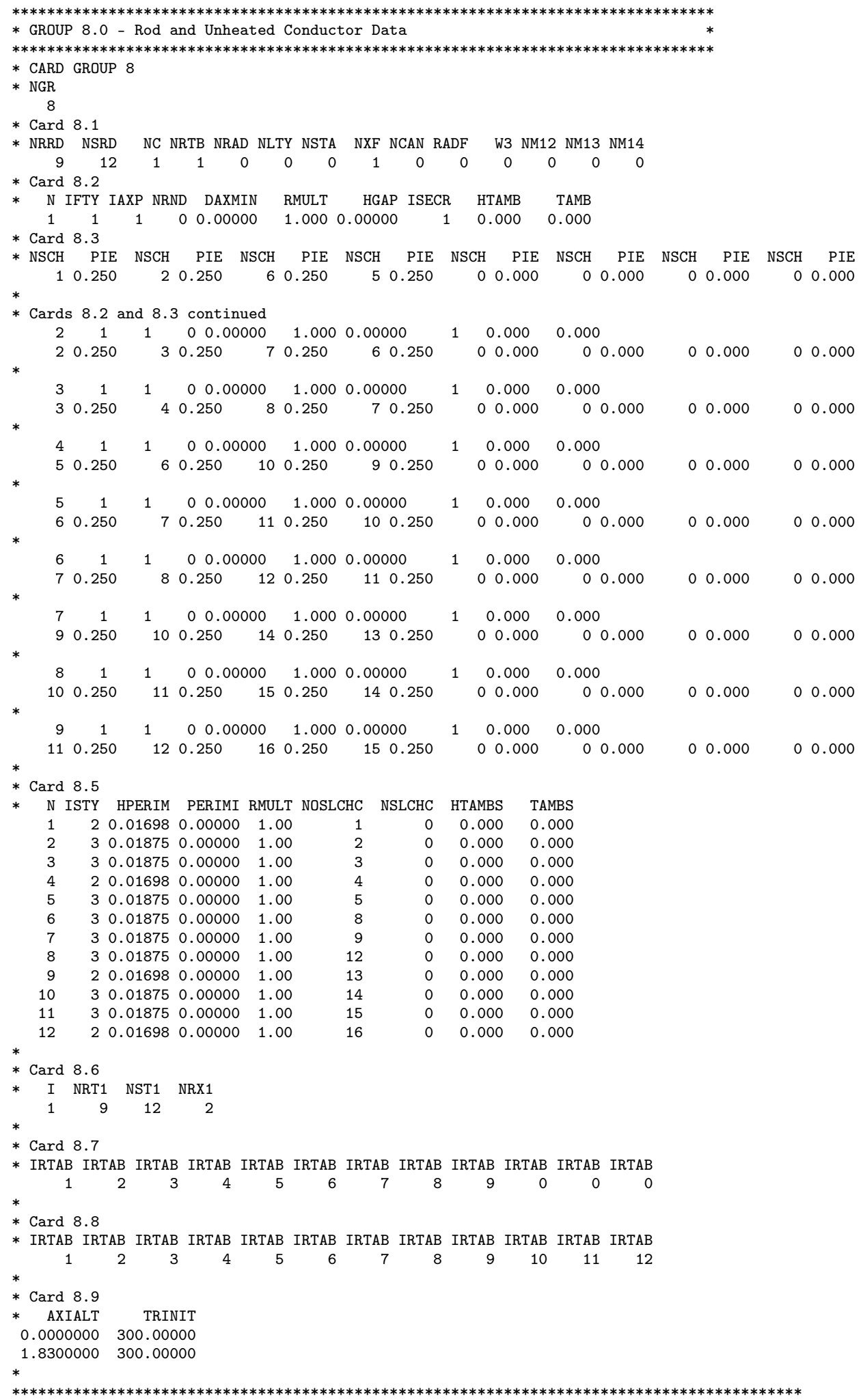




\subsubsection{Instructions to CARD GROUP 9}

The input for CARD GROUP 9 describes the characteristics of geometry types identified in the IFTYP(N) and ISTYP $(\mathrm{N})$ arrays for rods and unheated conductors in CARD GROUP 8. Geometry types fall into two basic classes âĂŞ nuclear fuel rods and all other conductors. The nonnuclear conductors can be characterized as solid cylinders, hollow tubes or flat plates.

On CARD 9.1, the user must specify total number of geometry types, NFUEL. This must be equal to the number of unique entries in the IFTYP(N) and ISTYP(N) arrays. Two flags for fuel relocation, IRELF and ICONF, are also input on CARD 9.1. Relocation is an option in the dynamic gap conductance model. The last entree is the flag for metal-water reaction, IMWR. The option is applicable only for Zirconium dioxide.

CARDS 9.2 through 9.5 are related to the nuclear fuel rod geometry only and must be omitted if fuel rods are not modeled. More than one nuclear fuel geometry type can be specified. The input on CARD 9.2 sets the geometry type index, I, which corresponds to the IFTYP(N) value of the rods that are of this geometry type. The geometry flag FTYPE(I) for nuclear geometry types is NUCL. The geometry of the nuclear fuel rod type is defined by the outside diameter, DROD; the pellet diameter, DFUEL(I); the diameter of the central core, DCORE (zero if the fuel is solid); and the clad thickness, TCLAD. The code assumes that the rod is uniform in axial direction, so these data completely characterize the physical dimensions of the rods being modeled by this geometry type.

On CARD 9.2, NFUEL defines the number of radial heat transfer nodes in the fuel pellet. NFUEL must be large enough for the code to resolve the temperature profile in the fuel pellet adequately, but more nodes specified will increase the time and space expenses when solving the heat conduction calculations. NFUEL between 5 and 10 is a good approximation for a fuel pellet diameter in the range of $1.0 \mathrm{~cm}$, but the user must exercise a degree of judgment in determining the number of radial nodes appropriate for the problem.

The remainder of the CARD 9.2 sets flags and options for the fuel material properties and the gap conductance model. The code contains properties for $\mathrm{UO}_{2}$ fuel derived from MATPRO-11 (Revision 1). These can be flagged by setting IMATF to zero. The correlations from MATPRO-11 use the fuel theoretical density, FTDENS(I), as a parameter in the calculation of the $\mathrm{UO}_{2}$ properties, which must be supplied by the user even if IMATF is zero. FTDENS (I) is a fractional value, that depends on the properties of $U_{2}$ pellets being modeled and is usually on the order of 0.95. Alternatively, the user may select to specify a different material for the fuel, in which case IMATF must be set to the numerical index of a material property type specified in CARD GROUP 10. For cladding material properties, the code contains properties of Zircaloy and Zircaloy oxide from MATPRO-11 (Revision 1) and these can be flagged by setting IMATC(I) and IMATOX (I) to zero. If the cladding or cladding surface oxide is some other material for the nuclear rods being modeled by geometry I, IMATC(I) and IMATOX(I) must be assigned the numerical index of an appropriate material type specified in CARD GROUP 10.

For the example shown in Figure 23.21, the input data for a nuclear fuel rod with constant gap conductance will have the following format: 


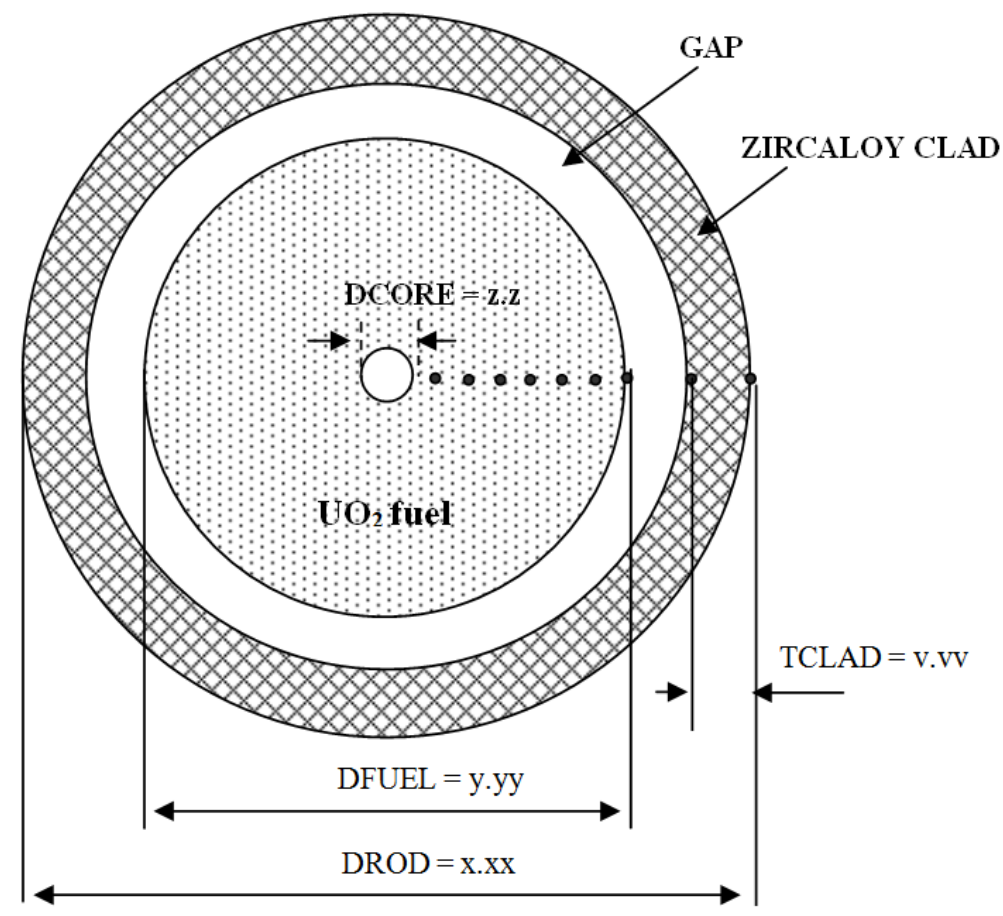

Figure 23.21: Nuclear fuel rod geometry

* Card 9.1

* NFUELT IRELF ICONF IMWR NDM5 NDM6 NDM7 NDM8 NDM9 NM10 NM11 NM12 NM13 NM14

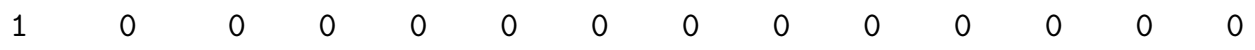

* Card 9.2: Nuclear Fuel Rod

* I FTYPE DROD DFUEL NFUEL IMATF IMATC IMTOX DCORE TCLAD FTDENS IGPC IGFORC IRADP

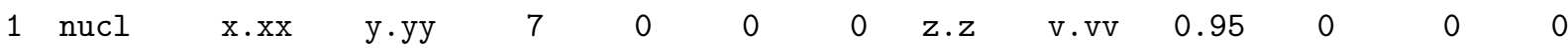
$*$

There are three options available for the gap conductance model for geometry type I:

1. constant uniform gap conductance;

2. axially non-uniform user-specified gap conductance; and

3. a dynamic gap conductance model

The simplest of the three alternatives, and the easiest to implement is the constant uniform gap conductance. It is flagged by setting IGPC to zero. The gap conductance value specified on CARD 8.2 for HGAP(N) is used for rods with geometry type I and no further input is required. 
The second option for a user-specified non-uniform gap conductance is a variation of the first option. The user specifies the value for gap conductance but can vary it axially and in time by means of input tables. The user specifies a table of pairs of values of axial location, AXJ (L), and gap conductance, AGFACT (L, I) on CARD 9.4. The number of pairs in the table is defined by the positive integer assigned to IGAPC on CARD 9.2. The code determines the gap conductance in each axial node of the rods with geometry type I by linear interpolation in the table specified on CARD 9.4 and applies the gap conductance temporal forcing function, if one has been specified (the temporal forcing function is specified on CARD 11.6). An example is given below:

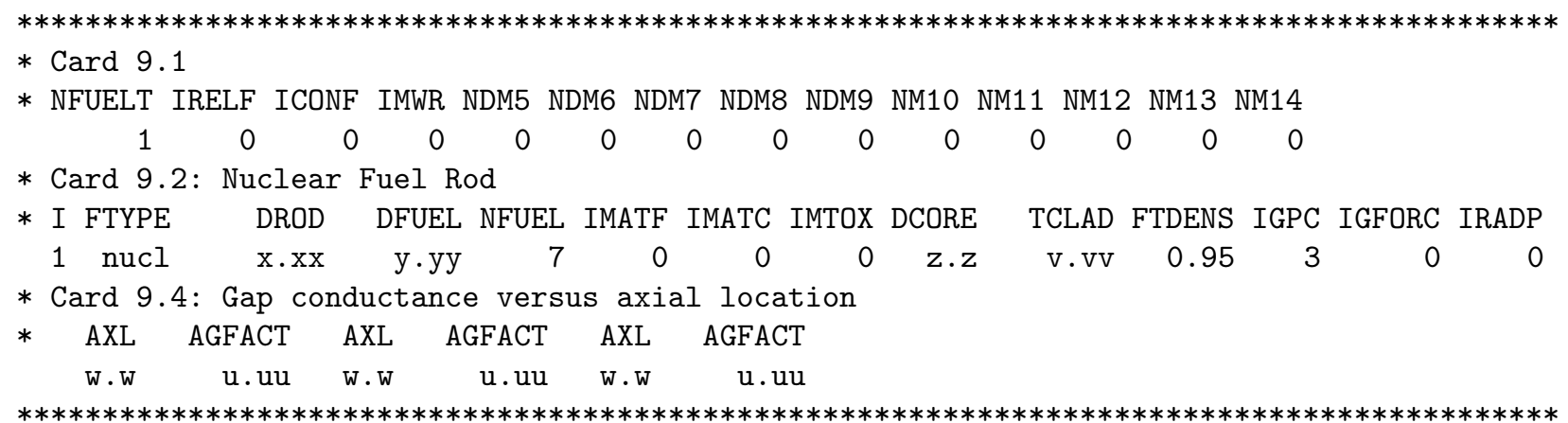

In the dynamic gap conductance model, the code calculates the gap conductance based on the thermal and geometric properties in the gap. The model takes into account the thermal expansion of the fuel pellet and cladding and elastic deformation of the cladding in determining the size of the gap. The dimensions of the gap and the thermodynamic properties of the fill gas are used to calculate the gap conductance. The user must specify the initial cold gap width in a table of axial location, AXJ (L), versus cold gap width, AGFACT(L, I), read on CARD 9.4. The cold gap width is the gap width before the fuel pin is brought to full power. But since the gap conductance model does not calculate the effects of the power history of the rod, the cold gap width specified by input must indicate any changes from as-built conditions due to burnup-dependent factors. The number of entrees in the table read on CARD 9.4 is the absolute value of IGPC (IGPC is entered as a negative number to flag the dynamic gap conductance model).

In addition to the cold gap width, the user must supply data on composition of the fill gas and the internal characteristics of the gap for the dynamic gap conductance model. These data are read on CARD 9.3. The cold pin pressure, PGAS (I), is the gas pressure at $295 \mathrm{~K}$. The gas plenum volume, VPLEN(I), is the volume of extra space between the top of the fuel pellet stack and the top of the rod. Both PGAS(I) and VPLEN(I) should be determined from the manufacturing specifications of the particular fuel rods being modeled by geometry I. The surface roughness of the fuel pellet and the cladding inner surface, ROUFF(I) and ROUFC(I), are used in the temperature jump discontinuity correlation in the gap conductance model. The correlation was optimized with values of $0.2 \times 10^{-4}$ inches and $0.39 \times 10^{-4}$ inches for ROUFC and ROUFF, respectively, and even if the user knows the correct values, the optimized values will likely yield a more accurate answer.

The fuel relocation model [5] can be included in the dynamic gap conductance calculations. Relocation allows fuel to move radially into the fuel-cladding gap. Cracks formed in the fuel by relocation reduce the effective conductivity of the fuel. When IRELF on CARD 9.1 is set to one, both radial relocation and conductivity degradation are included in the calculation. Since the relocation model is an empirical correlation, the surface roughnesses should correspond to those used to derive the correlation. ROUFC and ROUFF are $0.45 x 10^{-4}$ inches 
and $0.85 \times 10^{-4}$ inches, respectively in [3]. (These values are different from the surface roughnesses used to optimize the temperature jump discontinuity correlation). Conductivity degradation can be calculated without relocation by setting ICONF $=1$ and IREFL $=0$. In this case the effect of radial relocation should be included in the specified cold gap width.

The code expects the fill gas to be composed of helium, xenon, argon, krypton, hydrogen and nitrogen. The composition of the gas for a given geometry type is specified by filling the GSRAC(I) array on CARD 9.3 with values for the molar fractions (GSFRAC $\leq 1$ ) of the gases present. The GSFRAC(I) value for any one component can be any value between zero and one, inclusive, but the array must sum to unity:

$$
\sum_{L=1}^{6} \operatorname{GSFRAC}(\mathrm{L})=1.000
$$

For example, if the fill gas were 100\% helium, GSFRAC(1) would be 1.00 and GSFRAC(2) through GSFRAC(6) would all be 0.00 .

An example for a nuclear fuel rod with dynamic gap conductance, a gap filled only with helium, and no fuel relocation is given below:

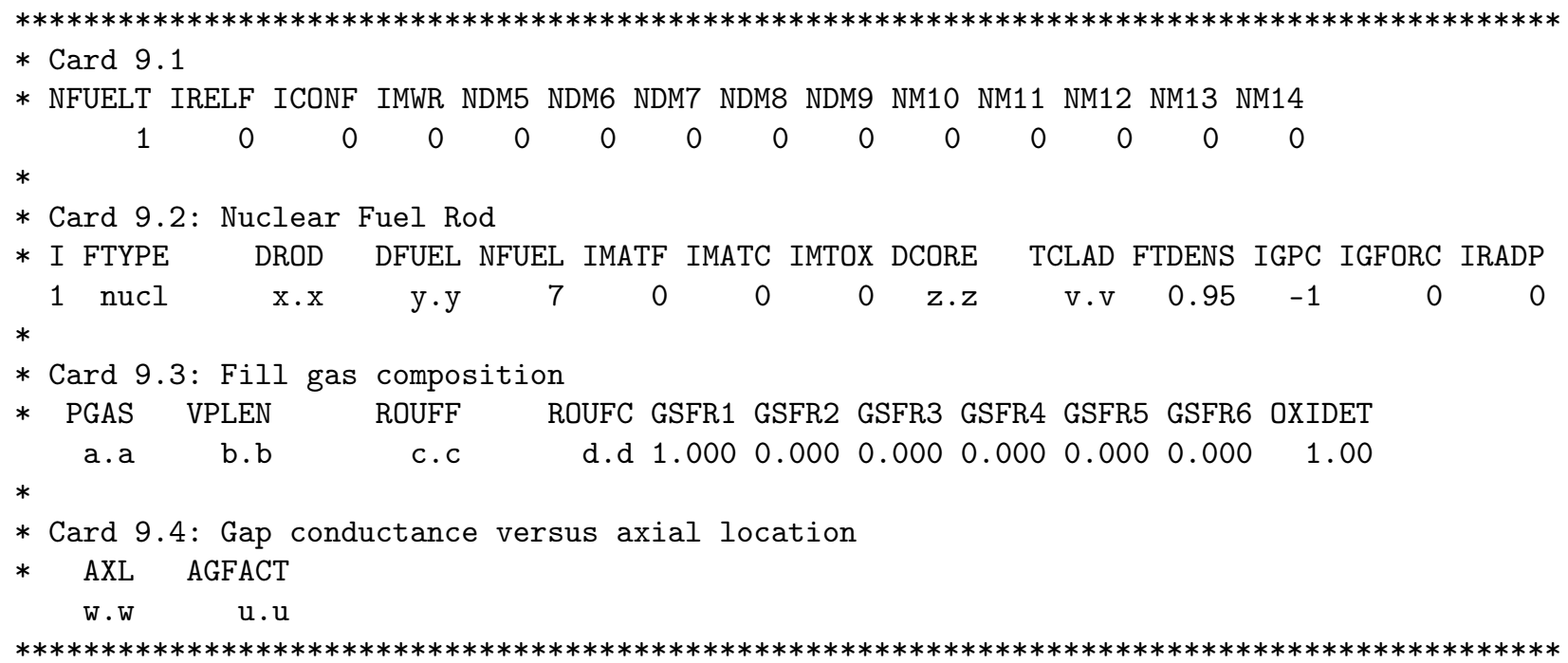

The code assumes that the radial power profile in a fuel pellet is uniform, but the user has the option of specifying a non-uniform radial profile in the pellet. If this option is selected, the pin radial power profile is read on CARD 9.5 as a table of relative radial location, RODP(L), versus relative power factor, POWR (L). The relative radial location is defined as:

$$
\mathrm{RODP}(\mathrm{L})=\frac{2\left(\mathrm{R}(\mathrm{L})-\frac{\mathrm{DCORE}}{2}\right.}{\text { DFUEL-DCORE }}
$$


where DCORE is the diameter of center void (in inches); DFUEL is the pellet diameter (in inches); and R(L) is the radial location (in inches).

The relative power fraction is defined as:

$$
\operatorname{POWR}(\mathrm{L})=\frac{\text { poweratradiallocation } \mathrm{R}(\mathrm{L})}{\text { averagepinpower }}
$$

The profile constructed by the table of (RODP (L), POWR(L)) is automatically normalized to unity.

CARDS 9.6 and 9.7 are related to the nonnuclear rod and unheated conductor geometry types. The geometry types are classified as solid cylinders, hollow tubes or flat plates. The physical elements of a reactor vessel or test section can be modeled with these geometries, even if they do not conform exactly to the ideal shape. A guide tube, for example, can be modeled as a flat wall, even though it is a tube. In modeling a solid element as a rod or unheated conductor, the important aspects to preserve are:

1. the surface area in contact with the fluid;

2. the mass available for thermal storage;

3. the thickness of any element (wall or tube) that has fluid contact on both surfaces

The physical dimensions of the geometry types should be defined so that these three characteristics of the solid elements of the system are modeled with reasonable accuracy.

The nonnuclear geometry types are defined on CARD 9.6 by index number I and an alphanumeric value for $\operatorname{FTYPE}(I)$ :

HROD for solid cylinder;

TUBE for hollow tube, and

WALL for a flat plate

The dimensions of the rods or unheated conductors modeled by a geometry type $I$ are defined by DROD and DIN. For solid cylinder geometry, DROD is the outside diameter and DIN is zero. For a tube geometry, DROD is the outside diameter and DIN is the inside diameter. For flat plate geometry, DROD is the perimeter of the plate surface that is in contact with the fluid and DIN is the plate thickness.

In many problems, DROD and DIN will correspond exactly to the physical dimensions of the elements being modeled. However, consider a square guide tube that is being modeled. It could be represented by an equivalent conductor, such a flat plate. The guide tube, with width $W$ and thickness $t$, has an outer diameter equal to $4 \mathrm{~W}$. A flat plate with equivalent outer perimeter would have $\mathrm{DROD}=4 W$ and $\mathrm{DIN}=t$. (In this case the user has to remember that an unheated conductor can be connected to only one fluid subchannel. If the guide tube from the above example is connected to more than one subchannel, it must be split to several walls with corresponding dimensions. See Figure 23.19).

The number of different material regions modeled in the rods or unheated conductors of geometry I is specified by the value of NFUEL on CARD 9.6. The number of heat transfer nodes in a region is specified 
by input on the subsequent CARD 9.7 input. A region defines a radial ring (or layer, for walls) of uniform material properties for the conduction solution. For example, a heater - composed of an electrical heater wire made from nichrome, a boron silicate filler material, and a stainless steel cladding — consists of three different materials: nichrome; boron silicate; and stainless steel. Four separate regions of materials are formed by the rod geometry: the central core of boron silicate; the nichrome heater wire; the outer layer of boron silicate; and the stainless steel cladding. Therefore, four material regions (NFUEL $=4$ ) are required to describe this heater rod geometry type.

The remaining input for CARD 9.6 defines the material properties used to determine the minimum film boiling temperature for the heat transfer surfaces of the geometry type. Most physical systems in contact with water, particularly at high temperatures, develop an oxide layer that significantly affects the thermal properties of the surface. The user may specify the thermal properties of the oxide material on the outside and inside surface by setting IMATOX (I) and IMATIX(I) to the index number of a material properties table specified in CARD GROUP 10. (The variable IMATIX (I) applies only to TUBE or WALL geometries with fluid contact on the inside surface. For solid cylinders (HROD) and TUBEs or WALLs with fluid contact only on the outside surface, it is ignored). If a particular geometry type does not have oxide scale on its heat transfer surfaces, IMATOX (I) and IMATIX(I) should be assigned values that correspond to the material properties table for the material the rods or unheated conductors are composed of at the surface. If IMATOX(I) and $\operatorname{IMATIX}(\mathrm{I})$ are set to zero, the oxide property index defaults to the built-in zirconium dioxide property table.

The material composition and radial noding in the NFUEL regions identified for a geometry type I are read on CARD 9.7. The user must specify a material property table index for the region MATR (L), corresponding to a properties table supplied in CARD GROUP 10. The physical thickness of each region must be specified in the TREG (L) variable, with the number of radial models in that region, NODER (L). The code automatically calculates the size of each node within the region by dividing the region into NODER(L) nodes of equal thickness.

The material temperature is calculated at the center of mass of each node. The only exception to this placement occurs for a region where one edge forms a heat transfer surface in contact with the fluid. In this case, the node closest to the surface is given only one-half the nominal thickness. The node temperature is calculated at the surface rather than the center of the half-width node.

For the above described heater rod with four material regions (NFUEL $=4$ ), Figure 23.22 shows a typical temperature profile in the heater rod and the radial nodding in the various region required to resolve it. Region 1, the boron silicate in the center of the rod, must of necessity be at a uniform temperature in steady state, and, since it is a relatively small region, it can be modeled adequately with a single node. The nichrome wire fills a thin enough region in the heater rod to be considered almost a line source and cannot have a significant temperature gradient (because of the thermal properties of nichrome), so this region too can be modeled with a single node. The region between the heater wire and the cladding has a very gradual slope because of the thermal properties of boron silicate, so two nodes are enough to resolve it adequately. The region defined by the steel cladding is relatively thin, but because of the thermal properties of steel, the temperature profile may have a relatively steep slope here, so two nodes are required in this region. However, because of special data handling inside the code, the following restriction holds: $2 \leq \mathrm{NODER}(\mathrm{L}) \leq \mathrm{NFUEL}$.

The number of radial nodes to use within a region of geometry is governed by two factors:

1. the temperature gradient expected in the structure; and 


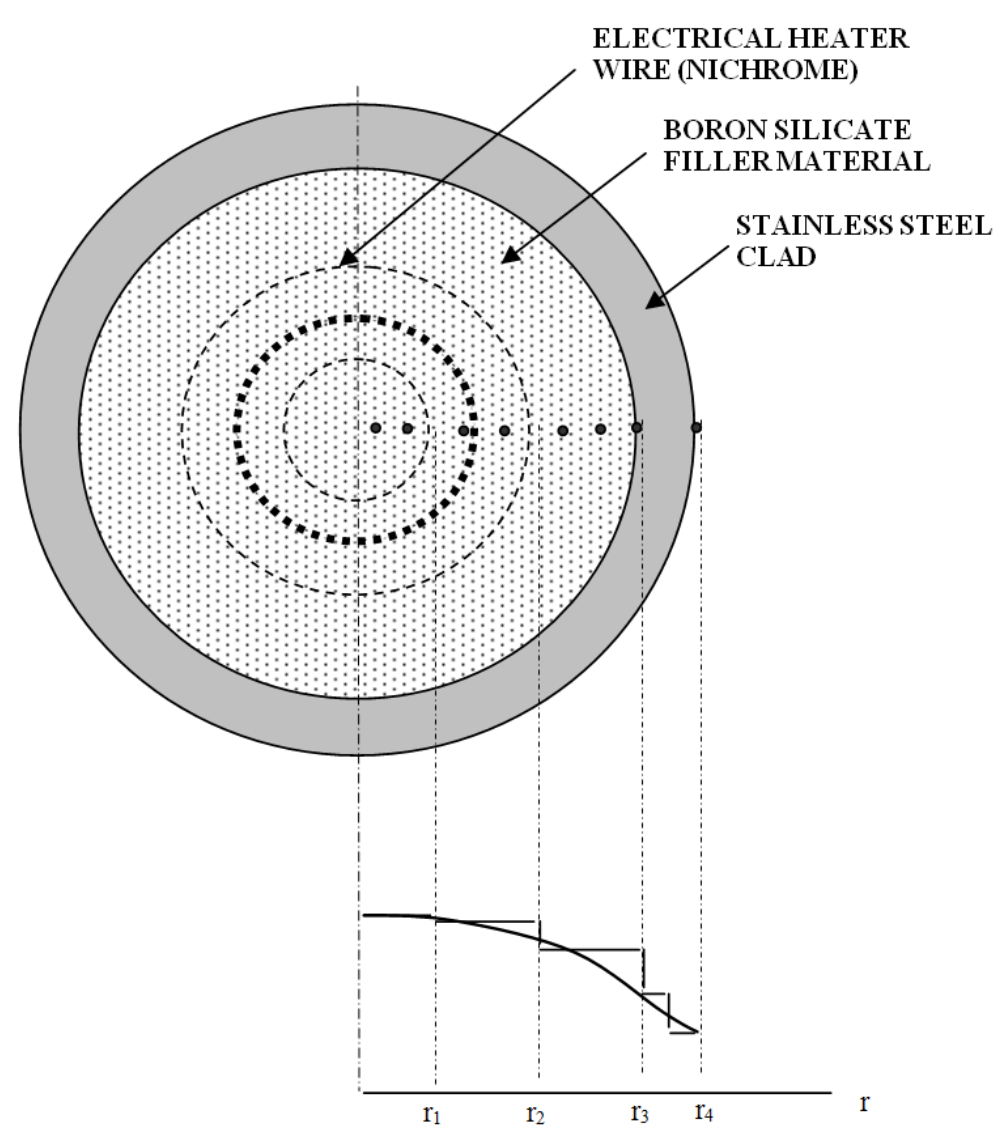

Figure 23.22: Heater rod geometry

2. the calculational cost for large number of heat transfer conditions being modeled.

Beyond a certain limit, however, doubling the number of nodes does not double the accuracy of the solution, but it nearly quadruples the computational time required for elements with that geometry type. The user must exercise some judgment as to what constitutes an adequate but not excessive number of radial nodes for the heat transfer solution in a particular problem.

The radial power factor, QREG (L), specified for each region, defines which regions of a nonnuclear rod generate heat internally. In heated rods, the QREG (L) values for all the regions of the rod are automatically normalized to unity, relative to the total power generated in a rod. Nonzero values of QREG are ignored in the unheated conductors.

The input for the heater rod shown in Figure 23.22 will be: 


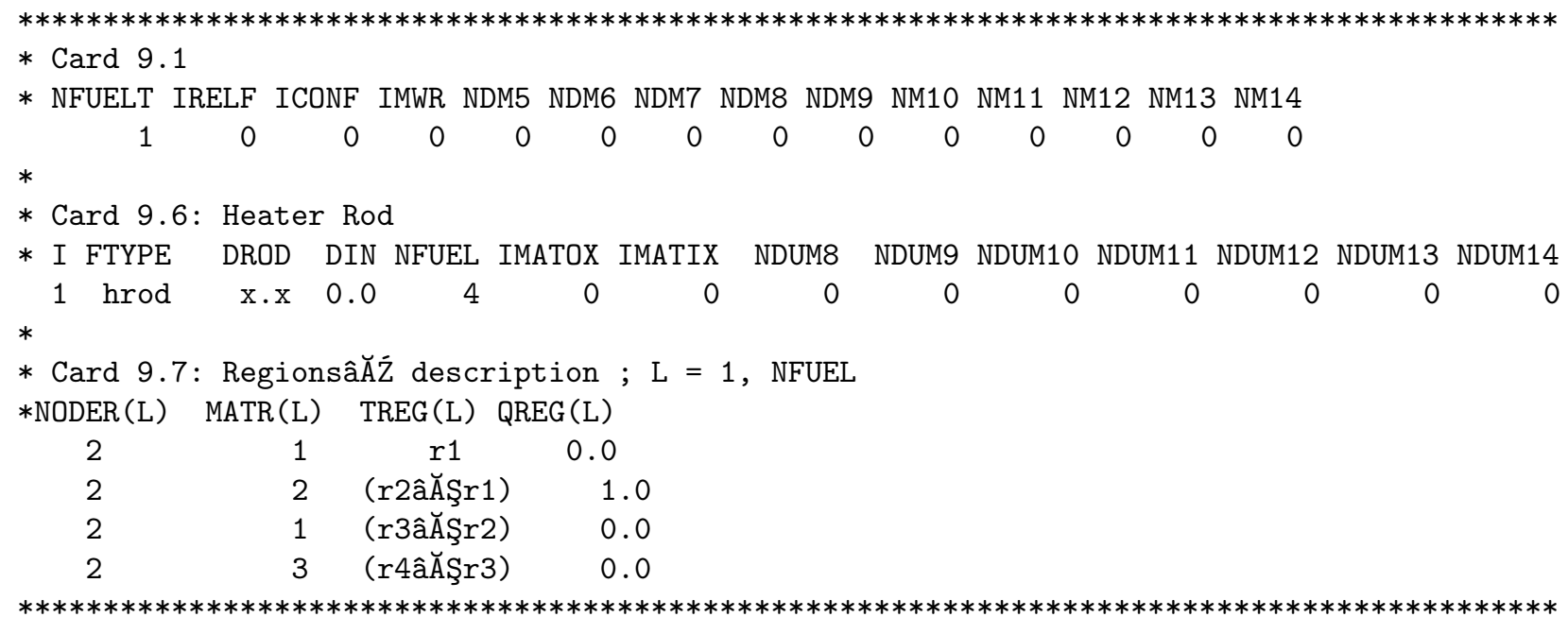

\subsubsection{Instructions to CARD GROUP 10}

The input for CARD GROUP 10 supplies the material properties selected by numerical indices in the geometry type input on CARD GROUP 9. If all the geometry types specified in CARD GROUP 9 use the build-in nuclear fuel rod properties for $\mathrm{UO}_{2}$, Zircaloy, and $\mathrm{ZiO}_{2}$, the input for CARD GROUP 10 is omitted. Otherwise, properties tables must be supplied for NMAT materials, where NMAT is the number of unique indices specified in CARD GROUP 9 input for the variables IMATF, IMATC, MATR(L), IMATOX(I), and IMATIX(I).

The material properties tables are entered on CARDS 10.2 and 10.3. Both cards are read for each table. CARD 10.2 identifies the table by its numerical index and specifies the number of entrees, NNTDP, in the table. The cold-state density of the material, RCOLD (N), is also entered on this card. RCOLD (N) is the density at normal (as-built) conditions and is used to calculated the mass of the nodes. This approach eliminates the tedious process of calculating the relocation of the radial nodes due to thermal expansion during the transient.

The properties table itself is read on CARD 10.3. The material properties included in the table are specific heat, $\operatorname{CPF}(I, N)$, and thermal conductivity, $\operatorname{THCF}(I, N)$, as functions of the temperature, TPROP(I,N). The temperature range of the table must be great enough to encompass the temperature extremes expected in the calculation; otherwise the run will abort due to property lookup failure. The user may specify constant properties for a material type. This is done by entering only one element in the table; i.e., setting NNTDP to 1, and reading CARD 10.3 for only one set of $\operatorname{CPF}(I, N), \operatorname{THCF}(I, N)$, and TPROP $(I, N)$. The values specified in $\operatorname{TPROP}(I, 1)$ is in this case is superfluous. The values specified in $\operatorname{CPFF}(I, 1)$ and $\operatorname{THCF}(I, 1)$ will be used for material type I regardless of the material temperature.

An example of the material properties input for a heated tube made from Inconel 600 is given below: 


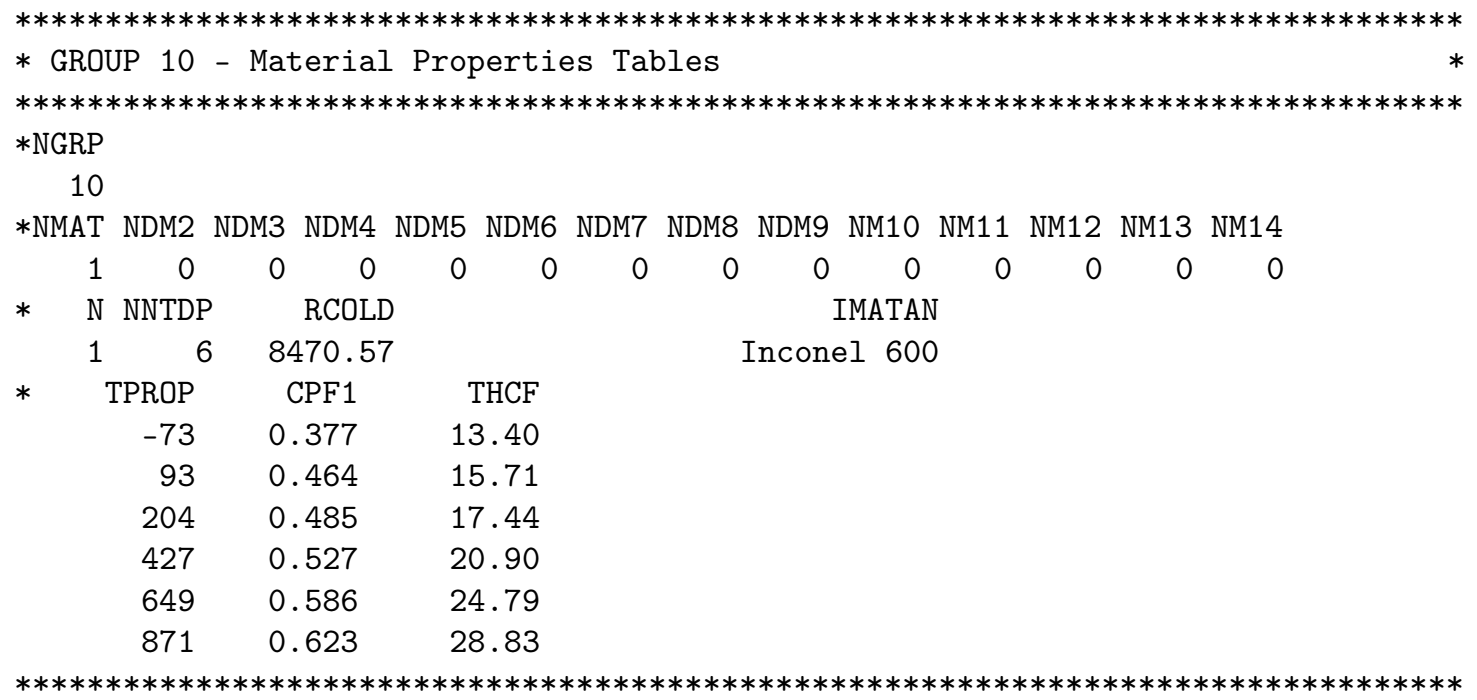

\subsection{Specification of the Initial and Boundary Conditions}

The next sections provide instructions to the specification of the initial and boundary conditions as well as the axial and radial power distribution. The initial operating conditions are read on CARD GROUP 1. The power distribution is prescribed on CARD GROUP 11. The boundary conditions are read on CARD GROUP 13 .

\subsubsection{Instructions to CARD GROUP 1}

The rod friction correlation, the entrainment and deposition model, the turbulent mixing and void drift models, and the pressure matrix solver to be utilized are selected via input on CARD 1.1. The number of non-condensable gases to be considered is specified by the parameter NGAS (maximum of eight). The rod friction correlation is chosen by the input flag IRFC. The modeling of entrainment and deposition at annular flow regime is activated by setting the input flag EDMOD equal to one. The turbulent mixing and void drift models are chosen by the input flag IMIX. The input flag ISOL selects the numerical solver for the system of pressure equations.

The initial operating conditions are specified in CARDS 1.2 and 1.3. The bundle (total) inlet flow rate, GTOT; the average linear heat rate, AFLUX; and the fraction of the heat rate generated directly in the coolant, DHFRAC, are read on CARD 1.2. The system pressure, PREF; the inlet fluid enthalpy, HIN (or the inlet temperature, TIN); the mass flux into the system, GIN; and the initial volume fraction of liquid in the liquid-vapor-gas mixture, VFRAC (1); and the initial volume fraction of vapor in the vapor-gas mixture, VFRAC(2) are read on CARD 1.3.

The system pressure, PREF, and enthalpy, HIN (or the enthalpy derived from the temperature: TIN), are used to determine the initial properties of the fluid. During the calculation the enthalpy and pressure change depending on the state of fluid and the local heat generation rate. The initial mass flux GIN is not, in 
general, compatible with the initial pressures, which are calculated based on gravity head losses only. The code must iterate to a correct solution. It is usually most expedient to specify the initial mass flux as zero; i.e., a "standing start".

If GTOT is set to non-zero value and the inlet boundary condition type (see CARD 13.4) is inlet mass flow rate and inlet enthalpy (BC type 2), the code will calculate subchannel mass flow rates according to the subchannels flow areas as specified on CARD 2.1 and will ignore the subchannel mass flow rates in CARD 13.4. If GTOT is set to zero and the inlet boundary condition type is inlet mass flow rate and inlet enthalpy (BC type 2), the user must specify subchannel mass flow rates in CARD 13.4.

If non-condensable gases are considered, their types (names) and volumetric fractions are specified on CARD 1.4 with the parameters GTYPE(I) and VFRAC(I+2), respectively. Valid non-condensable gas names include 'air', 'argo', 'heli', 'hydr', 'kryp', 'nitr', 'oxyg', and 'xeno'. Even without modeling non-condensable gases, at least one type (usually air) should be specified with negligibly small volumetric fraction. An example of CARD GROUP 1 is given below.

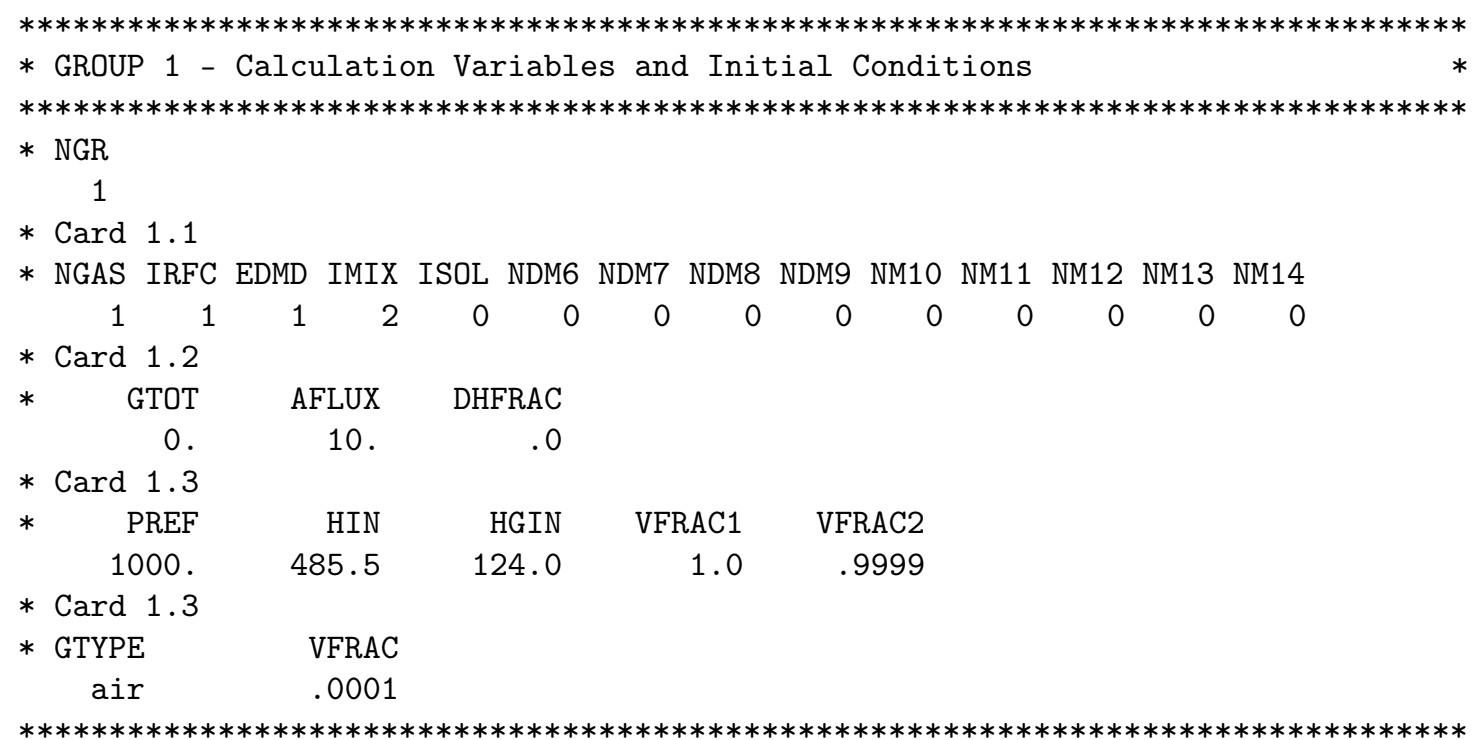

\subsubsection{Instructions to CARD GROUP 11}

The input for CARD GROUP 11 defines the steady state and transient two-dimensional power distribution. In addition, the user may specify temporal forcing functions on the total power generation rate and the nuclear fuel rods gap conductance. If no rods are specified in CARD GROUP 8 input, the input for CARD GROUP 11 is unnecessary and may be omitted entirely.

The CARD 11.1 specifies the number of transient changes of the axial profile, NQA (NQA must be equal to 1 for steady state calculations); the number of axial profile tables to be read for each transient point, NAXP (the identification numbers must correspond to the values specified in the IAXP array on CARD 8.2 and at least one table must be supplied); the maximum number of pairs of elements in any axial power profile table, MNXN (a minimum of two values, for the top and bottom of the rod, must be provided for the simplest case 
of an uniform power profile); the number of pairs of elements in the total power forcing function table, NQ (NQ is set to zero if total power is constant); the number of pairs of elements in the gap conductance forcing function table, NGPFF (NGPFF is set to zero if gap conductance is constant); and the number of radial profile tables, NQR (NQR corresponds to the transient points of time, for which different radial profiles will be read).

CARDS 11.2 through 11.4 are read NQA times. CARD 11.2 specifies the transient time, YQA, for which the axial power profile table will be read on the CARD 11.4. CARD 11.3 gives the identification number, I, and the number of pairs of elements, NAXN(I), in the axial power profile table I to be read in CARD 11.3. The table itself is read as NAXN (I) pairs of $Y(I, N)$ and $\operatorname{AXIALZ}(M, I, N)$, where $Y(I, N)$ is the axial location relative to the bottom and $\operatorname{AXIALZ}(M, I, N)$ is the power factor at that location. (Index $M$ varies from 1 to NQR). $\operatorname{AXIALZ}(M, I, N)$ is defined as the ratio of the local power to average power in the rod (rods) the table is applied to. Figure 23.23 shows a nuclear fuel rod with a $3.66 \mathrm{~m}$ active (heated) length and a chopped cosine power profile.

The axial power table is used to interpolate linearly for axial power factors at the boundaries of the axial heat transfer cells, and the profile is re-integrated over each cell to obtain on average linear heat rate for the cell. When re-noding occurs, the axial power profile is re-integrated to obtain the average linear heat rate in the new cells. In the example shown in Figure 23.24, an electrical heater rod with step approximation to a cosine power profile in modeled in a subchannel with $0.305 \mathrm{~m}$ continuity nodes. The linear heat rate in the cell is modeled as a uniform $Q_{i} n$ along entire length of the cell.

With subchannel splitting it is possible to extend a rod through several sections of channel geometry. A rod can be used to model an average heater rod in a test section, as illustrated in Figure 23.25. Channel 3 in Section 1 models a portion of the region penetrated by the cold ends of the rods modeled by the average heater rod 1. Channel 3 connects to Channel 10 above. Channel 10 in Section 2 models a portion of the core containing the rods modeled by Heater Rod 1. Channel 10 connects to Channels 15, 16, and 17 above, in Section 3, but the heater rod ends at the boundaries between Sections 2 and 3.

In general, a rod can pass through any number of contiguous vertical sections with fluid connections in different subchannels in each section, but the rod must begin and end at a section boundary. The vertical locations of the beginning and end of each rod must be considered when defining section boundaries in CARD GROUP 4. Unheated conductors do not require axial power profiles and may not cross section boundaries, but they must also be considered in the section boundary locations. Unheated conductors are assumed by the code to have the same axial length as the sections that contain them.

The total power forcing functions are read on CARD 11.5 in NQ pairs of transient time YQ(N) and power factor $F Q(N)$. The power factor $F Q(N)$ is the ratio of power at time $Y Q(N)$ and the initial power. The code interpolates linearly in the power forcing function table to determine the current value of total power at each step.

The gap conductance forcing functions are specified on CARD 11.6 as NGPFF pairs of transient time YGPFF (N) and gap conductance factor FGPFF (N). The input convention for this table follows the logic as the other tables in CARD GROUP 11. The gap conductance factor FGPFF(N) is defined as the ratio of the gap conductance value at the time $\mathrm{YGPFF}(\mathrm{N})$ and the initial value. The forcing function is applied uniformly along the axial length for nuclear fuel rods with a gap conductance forcing function specified on CARD 9.2 (IGPC >0).

The next two cards, CARDS 11.7 and 11.8, provide the radial power profile and its transient behavior. CARD 11.7 specifies the transient time, YQR, for which an array FQR (N), containing the radial power factors of rods (the local power generation in each individual rod), will be read in the CARD 11.8. 


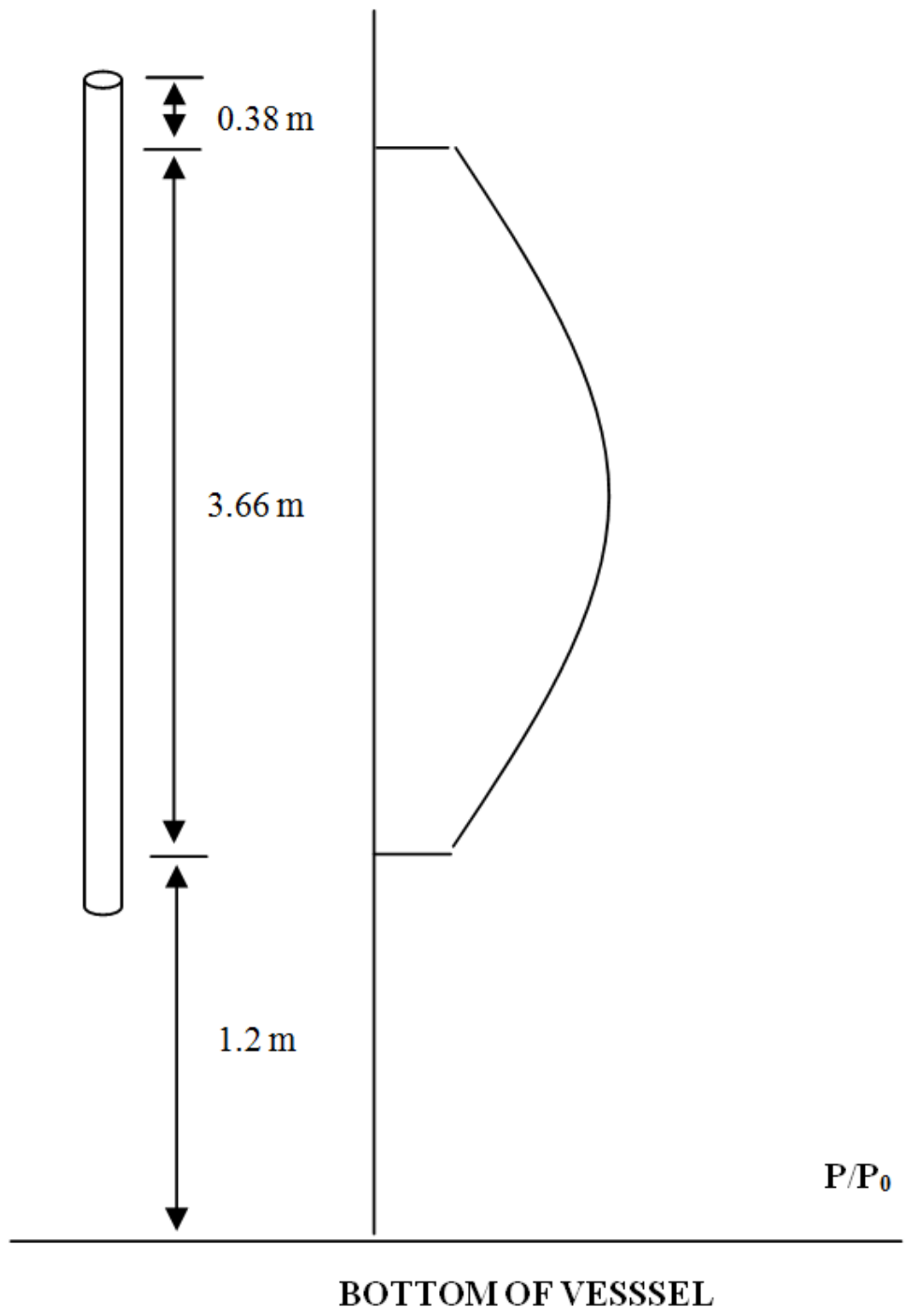

Figure 23.23: Nuclear fuel rod power profile 

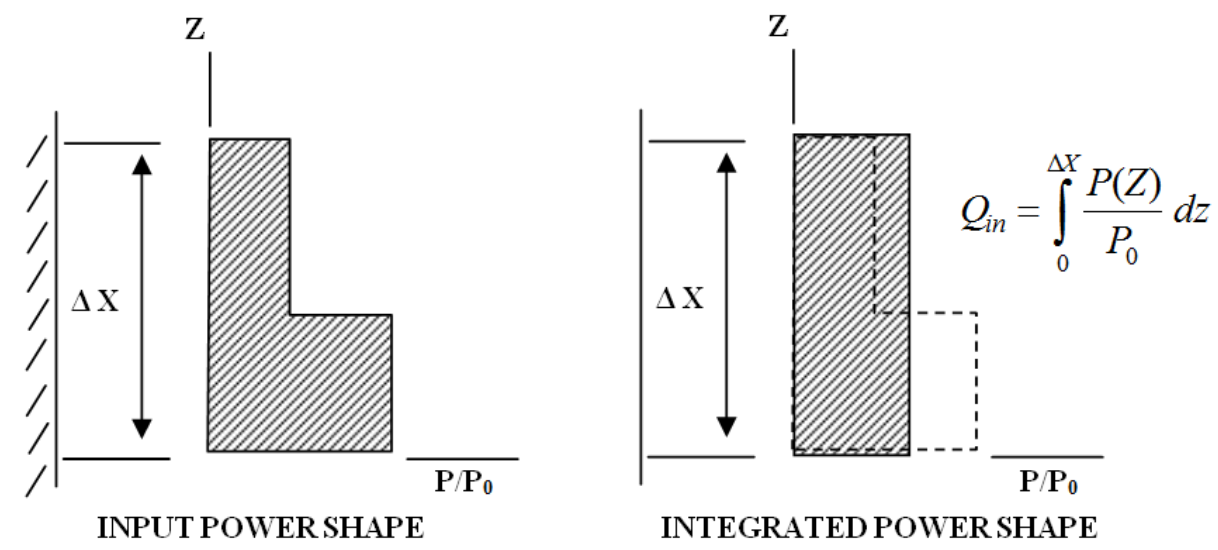

Figure 23.24: Heat input over one fluid node

The code calculates the local linear heat rate (which is transferred through the rod surface to heat up the coolant) as follows:

$$
\dot{q}(\operatorname{rod}, x, t)=(1-d) \dot{\bar{q}} f_{\text {time }}(t) f_{\text {axial }}(x, t) f_{\text {radial }}(\operatorname{rod}, t)
$$

where $\dot{\bar{q}}$ is the AFLUX read on CARD $1.2 ; d$ is the DHFRAC read on CARD $1.2 ; f_{\text {time }}(t)$ is the FQ read on CARD 11.5; $f_{\text {axial }}(x, t)$ is the AXIALZ read on CARD 11.4; and $f_{\text {radial }}(r o d, t)$ is the FQR read on CARD 11.8.

An example of input for CARD GROUP 11 for the configuration shown in Figure 23.20 with a constant uniform axial and radial power distributions and transient total power is given below. 


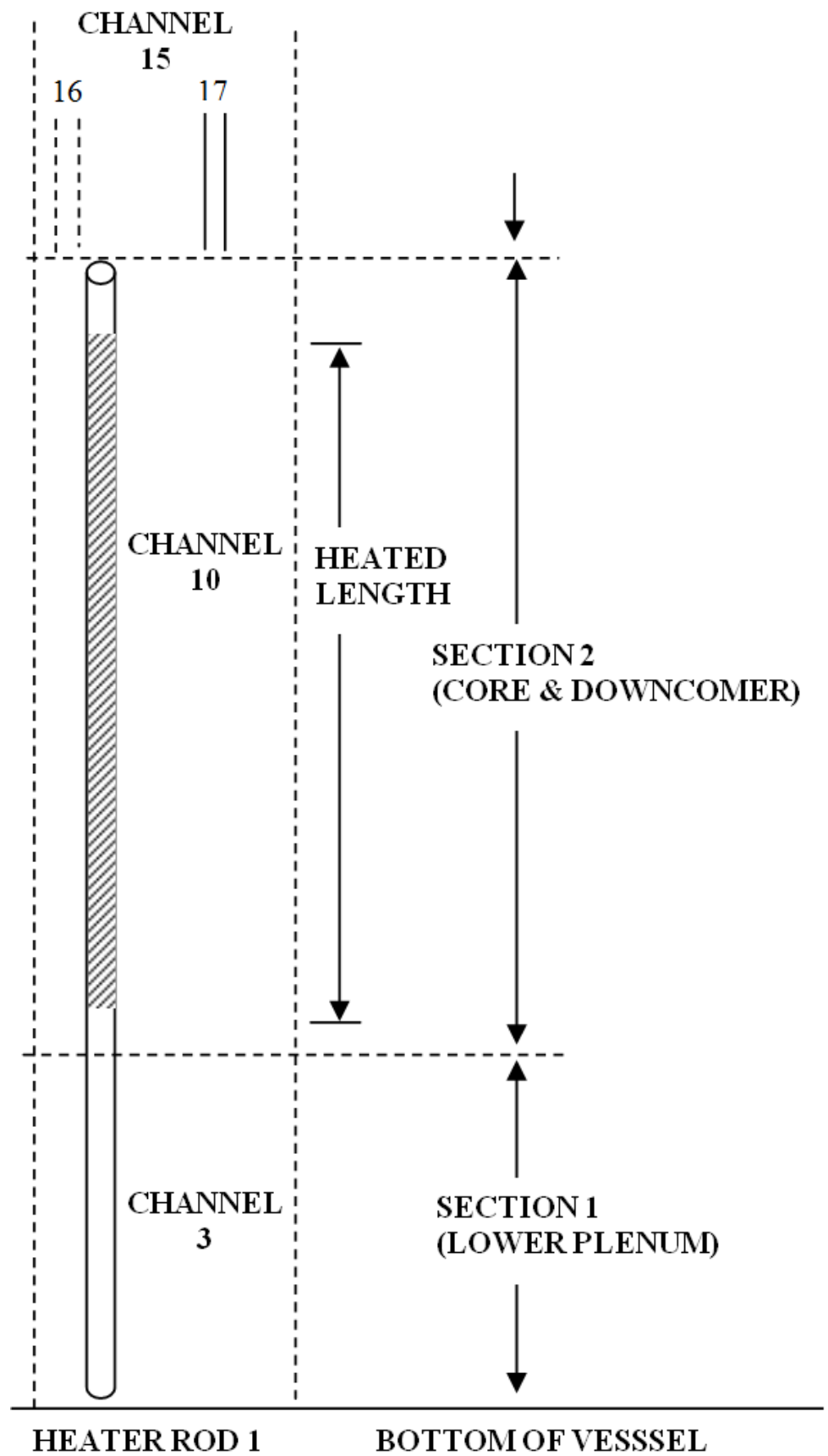

Figure 23.25: Heater rod crossing section boundaries 


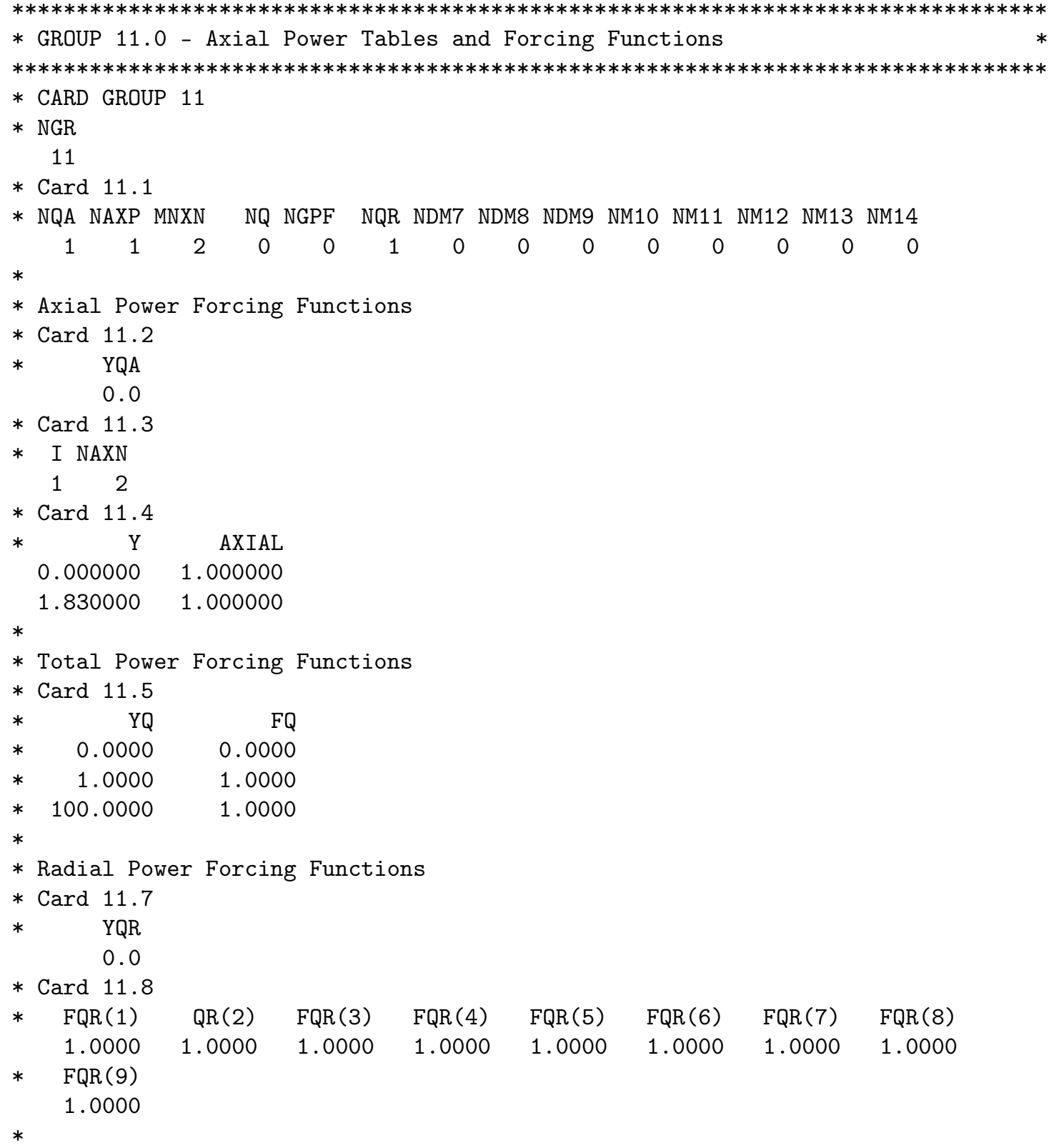

\subsubsection{Instructions to CARD GROUP 13}

Two main types of boundary conditions can be specified by the input in CARD GROUP 13. These are:

1. inlet or outlet boundary conditions on subchannels, and 
2. specific boundary conditions on particular cells within the mesh for both the vertical and transverse control volumes

Inlet or outlet boundary conditions must be specified for subchannels that do not have vertical connections to other subchannels in the system at their inlet or outlet. This, obviously, must include the inlet of subchannels in the first axial section and the outlet of subchannels in the last axial section. But other subchannels within the stack of sections can be unconnected at the top or bottom to other subchannels. Mesh boundary conditions may be applied wherever needed, on any face of any cell within the vessel mesh. But only one boundary condition can be applied to any one node.

The number of subchannel mesh cell boundary conditions, including inlet, outlet, and internal mesh cells, in a given problem is specified by the value of NIBND on CARD 13.1. The NIBND boundary conditions are set by reading CARD 13.4 for each boundary, specifying the subchannel number, IBOUND (1,M); axial node number, $\operatorname{IBOUND}(2, \mathrm{M})$; boundary type number, ISPEC (M); the first boundary value, BCVALUE1; the second boundary value, BCVALUE2; and the third boundary value, BCVALUE3. The forcing functions for a given boundary condition type are identified by table number in parameters N1FN, N2FN, and N3FN for the first, second and third boundary values, respectively. The boundary conditions that can be specified for subchannel mesh cells are listed in Table 10.

The general application of the boundary conditions is summarized in Table 11.

If boundary condition type 1 is selected, the pressure in subchannel $\mathrm{I}=\operatorname{IBOUND}(1, \mathrm{M})$ at node $\mathrm{J}=$ $\operatorname{IBOUND}(2, \mathrm{M})$ is set to BCVALUE3 and the enthalpy is set to BCVALUE2. BCVALUE1 is not used. For boundary condition type 2 , the flow at the top face of node $\operatorname{IBOUND}(2, \mathrm{M})$ in the $\operatorname{subchannel} \operatorname{IBOUND}(1, \mathrm{M})$ is $\operatorname{set}$ to BCVALUE1 and the enthalpy is set to BCVALUE2. BCVALUE3 is not used. The flow may be positive or negative. For boundary condition type 3, the flow is set to BCVALUE1. BCVALUE2 and BCVALUE3 are not used. This boundary condition is used to shut off vertical flows within the mesh or to specify flow into or out of the top of a subchannel, where it is not desirable to specify the node enthalpy, as is done for boundary condition type 2. Boundary condition type 4 is used to specify a mass source in any cell. BCVALUE1 is set to the mass flow rate, BCVALUE2 is set to the enthalpy, and BCVALUE3 is set to pressure. Boundary condition type 5 is used to specify a pressure sink connected to any mesh cell. BCVALUE3 is the pressure sink, and BCVALUE2 is the enthalpy of the fluid in the sink. BCVALUE1 is not used.

Table 10: Boundary Condition Types

\begin{tabular}{|l|l|c|c|l|}
\hline \multicolumn{2}{|l|}{ Boundary condition type } & Internal mesh cells & Boundary cells & Input in CARDs \\
\hline 1 & Pressure and enthalpy & - & $\mathrm{X}$ & $13.4,13.6,13.7$ \\
\hline 2 & Mass flow rate and enthalpy & - & $\mathrm{X}$ & $13.4,13.6,13.7$ \\
\hline 3 & Mass flow rate & $\mathrm{X}$ & - & 13.4 \\
\hline 4 & Mass source & $\mathrm{X}$ & - & $13.4,13.5,13.6,13.7,13.8$ \\
\hline 5 & Pressure sink & $\mathrm{X}$ & - & $13.4,13.6,13.7,13.9$ \\
\hline- & Crossflow set to zero & $\mathrm{X}$ & - & 13.10 \\
\hline
\end{tabular}

In addition to the axial boundary conditions, the transverse flow between subchannels connected by a gap can be shut off at any elevation within the vessel by specifying input for CARD 13.7. This input is read 
if parameter NKBND on CARD 13.1 is greater than 0. The input for CARD 13.7 consists simply of the gap identification number $K$ and the contiguous axial levels JSTART to JEND where the crossflow will be set to zero. This format makes it very easy to zero out a sequence of crossflows, from $J=2$ to $J=15$, for example.

If flow is to be shut off in more than one segment of a gap, CARD 13.7 input can be specified for gap $K$ more than once, until all of the axial levels to be zeros have been specified.

Unless otherwise indicated, the special boundary conditions applied to cells as specified in CARD GROUP 13 are constant in time. The user may, however, specify forcing functions on BCVALUE1, BCVALUE2, and BCVALUE3 for any or all of the special boundary conditions. The number of forcing functions is specified by the value of NFUNCT on CARD 13.1.

The forcing functions on the special boundary conditions are specified in the same way as any other forcing function in the vessel input - as tables of forcing function factors versus time. The number of elements in a given table is read in CARD 11.2 as NPTS; then NPTS pairs of ABSCIS $(K, I)$ and ORDINT (K, I) are read on CARD 13.3. The ABSCIS array contains the transient time, in seconds, when the corresponding forcing function factor ORDINT is to be applied. ORDINT is defined as the ratio of the value at time ABSCIS (K, I) of the parameter being forced and its initial value. The factor is determined at any given time step by linear interpolation in the forcing function table. The forcing function tables are numbered sequentially from 1 to NFUNCT in the order they are read in.

In the code, the initial mass flow rate is specified as zero in the whole flow domain by default. That means a "standing start" is performed. (Otherwise there could be severe problems with convergence). For this reason the inlet mass flow rate is also highly recommended to start as zero and to be run up by means of a ramp which can be specified by a forcing function (see variable N1FN). This concerns boundary condition types 2,3 , and 4 .

If a mass source boundary condition (type 4) has been specified for a cell on CARD 13.4, additional information must be supplied on CARD 13.5 specifying the droplet diameter DROPS $(\mathrm{N})$ and droplet mass flow rate FDROPRS (N) at the injection boundary. The index number of the forcing function table, NDFN (N), by which the droplet diameter will vary and the index number of the forcing function table, NDFFN (N), by which the droplet mass flow rate will vary must be provided as well.

For all types of boundary conditions except for type 3 the enthalpy of the non-condensable gases mixture, HMGA $(\mathrm{N})$, and their volumetric fractions, GVALUE $(\mathrm{NGA}, \mathrm{N})$, have to be specified on CARD 13.6. The corresponding forcing functions $\operatorname{NHMFN}(\mathrm{N})$ and $\mathrm{NGFN}(\mathrm{NGA}, \mathrm{N})$ are read on CARD 13.7. CARDS 13.6 and 13.7 are not needed and can be omitted by setting INITGAS on CARD 13.4 to 1 , when the inlet conditions for the non-condensable gases are considered equal to the initial conditions entered on CARDS 1.3 and 1.4 $($ HMGA $=$ HGIN; GVALUE $=$ VFAC; NHMFN $=0$; and NGFN $=0)$.

If a pressure sink boundary condition (type 5) has been specified for a cell on CARD 13.4, additional information describing the sink geometry must be supplied on CARD 13.9. Flow in or out of the sink is defined for a special control volume, as shown in Figure 23.26. The flow area of the control volume is ASINK and the length of the cell is DXSINK. The sink boundary condition usually models a real hole in the system and in this case ASINK is the area of the hole. The control volume length should be on the order of the centroid length of the channel in which the sink lies. The flow in and out of the sink generally encounters some unrecoverable pressure loss due to expansion at the orifice and the loss coefficient associated with the sink flow is defined by SINKK on CARD 13.9. The type 5 boundary conditions are numbered sequentially within the code in the order they are read in on CARD 13.4. CARD 13.9 should be read for the sink in the 


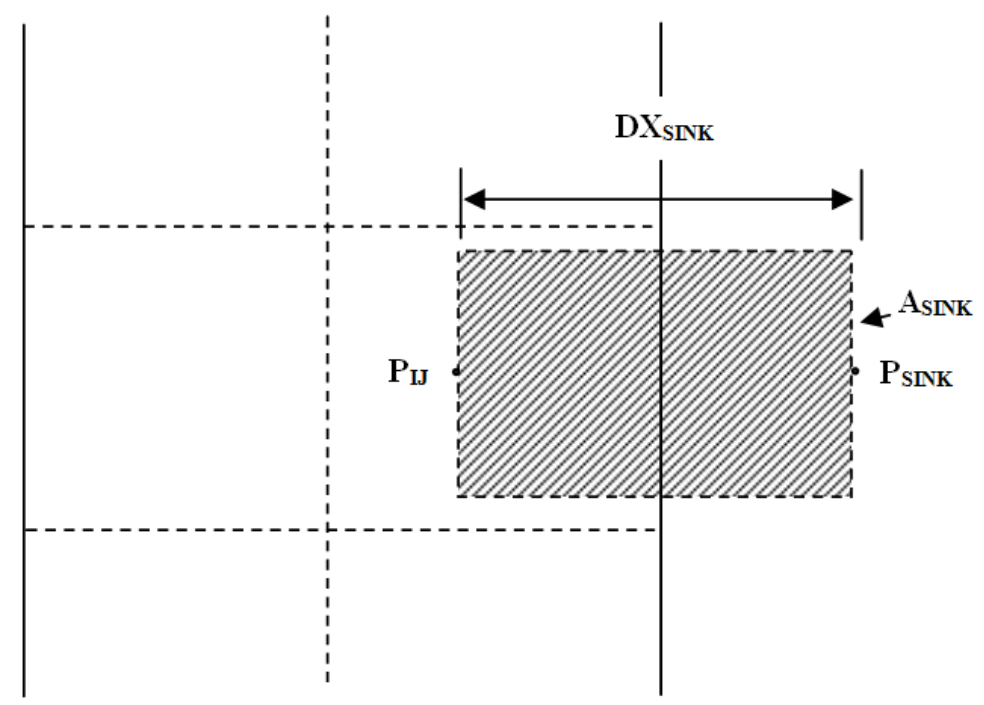

Figure 23.26: Control volume for pressure sink boundary conditions

same sequential order. CARD 13.8 is read for the flow area of mass sources in the same manner.

An example of input for CARD GROUP 13 for the configuration shown in Figure 23.20 at steady state conditions is given below. 


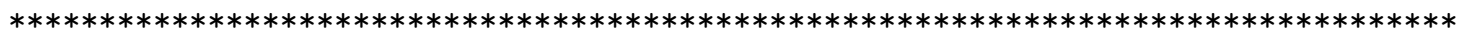

* GROUP 13.0 - Boundary Condition Data

$* * * * * * * * * * * * * * * * * * * * * * * * * * * * * * * * * * * * * * * * * * * * * * * * * * * * * * * * * * * * * * * * * * * * * * * * * * * * * * * * * * *$

* CARD GROUP 13

* NGR

13

* Card 13.1

* NBND NKBD NFUN NGBD NDM5 NDM6 NDM7 NDM8 NDM9 NM10 NM11 NM12 NM13 NM14

* Card 13.2

* NPT

4

* Card 13.3

* ABSC ORDINT

$0.0 \quad 0.000$

ABSC ORDINT ABSC ORDINT

$\begin{array}{llllll}0.1 & 0.000 & 0.2 & 1.000 & 1500.0 & 1.000\end{array}$

* Card 13.4

* Inlet b.c.

* IBD1 IBD2 ISPC N1FN N2FN N3FN

$\begin{array}{rrrrrrrrrr}1 & 1 & 2 & 1 & 0 & 0 & 0.0 & 918.77 & 0.0000 & 1 \\ 2 & 1 & 2 & 1 & 0 & 0 & 0.0 & 918.77 & 0.0000 & 1\end{array}$

. .

$\begin{array}{llllllllll}16 & 1 & 2 & 1 & 0 & 0 & 0.0 & 918.77 & 0.0000 & 1\end{array}$

* Outlet b.c.

* IBD1 IBD2 ISPC N1FN N2FN N3FN BCVALUE1

BCVALUE2

BCVALUE3 INITGAS

$\begin{array}{llllllllll}1 & 38 & 1 & 0 & 0 & 0 & 0.0000 & 918.77 & 70.00 & 1\end{array}$

$\ldots$

0.0000

918.77

70.00

1

$\begin{array}{llllllllll}16 & 38 & 1 & 0 & 0 & 0 & 0.0000 & 918.77 & 70.00 & 1\end{array}$

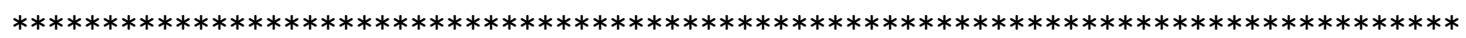




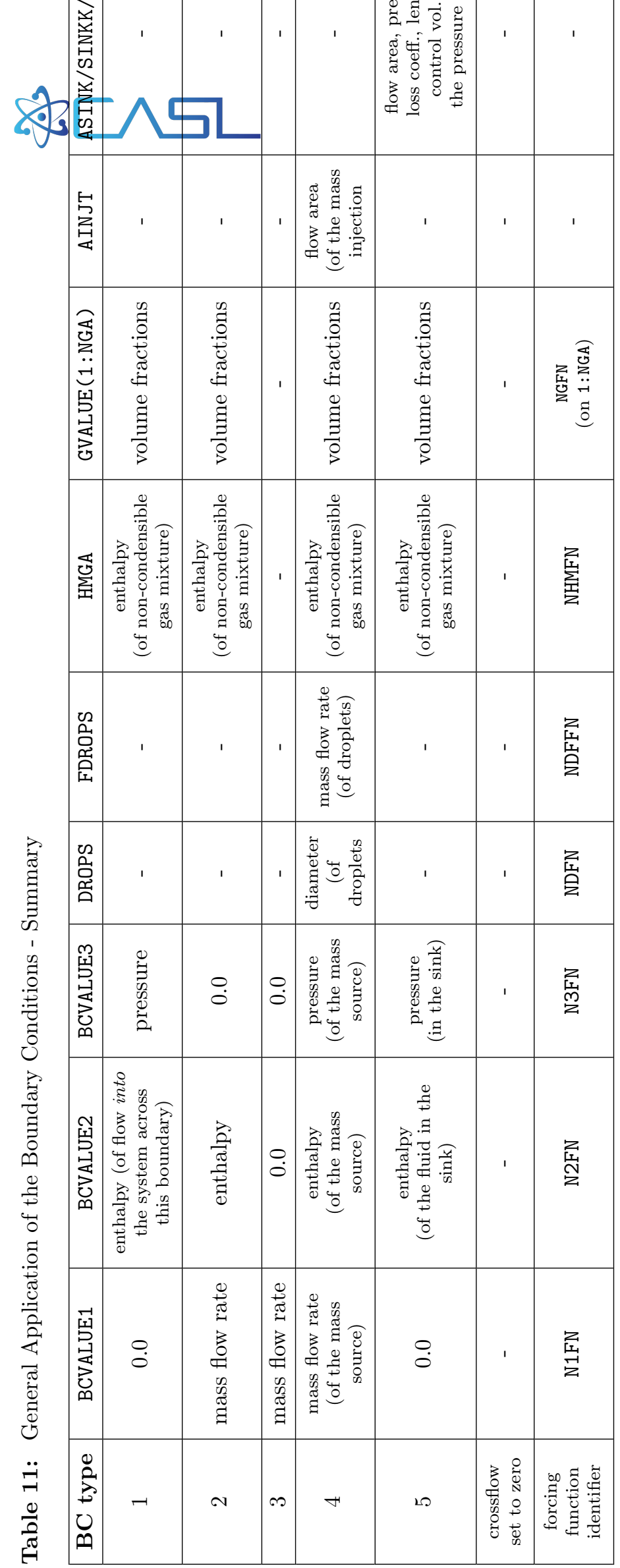




\subsubsection{Boron Tracking and Precipitation Modeling}

\subsubsection{Application of the Boron Tracking/Precipitation Model}

The boron tracking model is controlled in the CTF input deck along with the rest of the CTF user-defined parameters. All of the parameters related with the boron tracking and precipitation model are conditioned to the variable IBTM (defined in CARD 1.1), the flag of the model. The user can choose between the First Order Accurate Upwind Scheme and the Second Order Accurate Modified Godunov Scheme (explained in Section A).

Initial conditions of the boron are defined in CARD 1.3 where the initial boron concentration (BRIN) of the system is uniformly defined, and the boron physical diffusion coefficient (RDIF). The boron physical diffusion coefficient is only used when is applied the Second Order Accurate Modified Godunov Scheme (IBTM $=2$ ). The suggested values are: $0.0($ IBTM $=1)$ and $1.0($ IBTM $=2)$, due to RDIF is the result of experimental data.

The boron tracking model boundary conditions are defined in CARD 13.11. As with other CTF boundary conditions, forcing functions may be defined for the inlet boron concentration (CARDs 13.2 and 13.3) and the total number of vertical mesh cell boundary conditions, number of boron inlet boundary conditions (NIBNDB, in CARD 13.1); this value may be not the same as the number of inlet boundary conditions entered in CARD 13.4. This allows the user to control the inlet boron with respect to time as the CTF-modeled transient progresses.

CARD 13.11 follows the same scheme as CARD 13.4: $\operatorname{IBOUNDB}(1, \mathrm{~N})$, index number of channel at which boundary condition $\mathrm{N}$ is applied ; $\operatorname{IBOUNDB}(2, \mathrm{~N})$, vertical node number at which boundary condition $\mathrm{N}$ is applied; N4FNB (N), index number of the forcing function table by which the forth parameter of the boundary condition (BCVALUE4B) will be varied; and BCVALUE4B(N), forth boundary condition represents the boron concentration $[\mathrm{ppm}]$; where $\mathrm{N}=1$ : NIBNDB.

There must be a match between where the inlet boundary conditions and the boron inlet boundary conditions are applied, (IBOUND $(\mathrm{L}, \mathrm{N}), \mathrm{L}=1: 2)$ and $(\operatorname{IBOUNDB}(\mathrm{L}, \mathrm{N}), \mathrm{L}=1: 2)$.

The boron tracking/precipitation model is applied when ISPEC $(\mathrm{N})=1,2$ or 3 , what that means, except when a source $($ ISPEC $=4)$ or a sink (ISPEC $=5)$ is the boundary condition. The boron inlet boundary condition, BCVALUE4B, and its associated forcing function, N4FNB; are reassigned to the variables BCVALUE4(N) and N4FN (N), N=1:NIBND, as an extra boundary condition, based on the subchannel and axial level where the boron inlet boundary conditions are applied. 
23.4.4.2 Example of Card Group 1 and Card Group 13 (Boron Tracking/Precipitation Model)

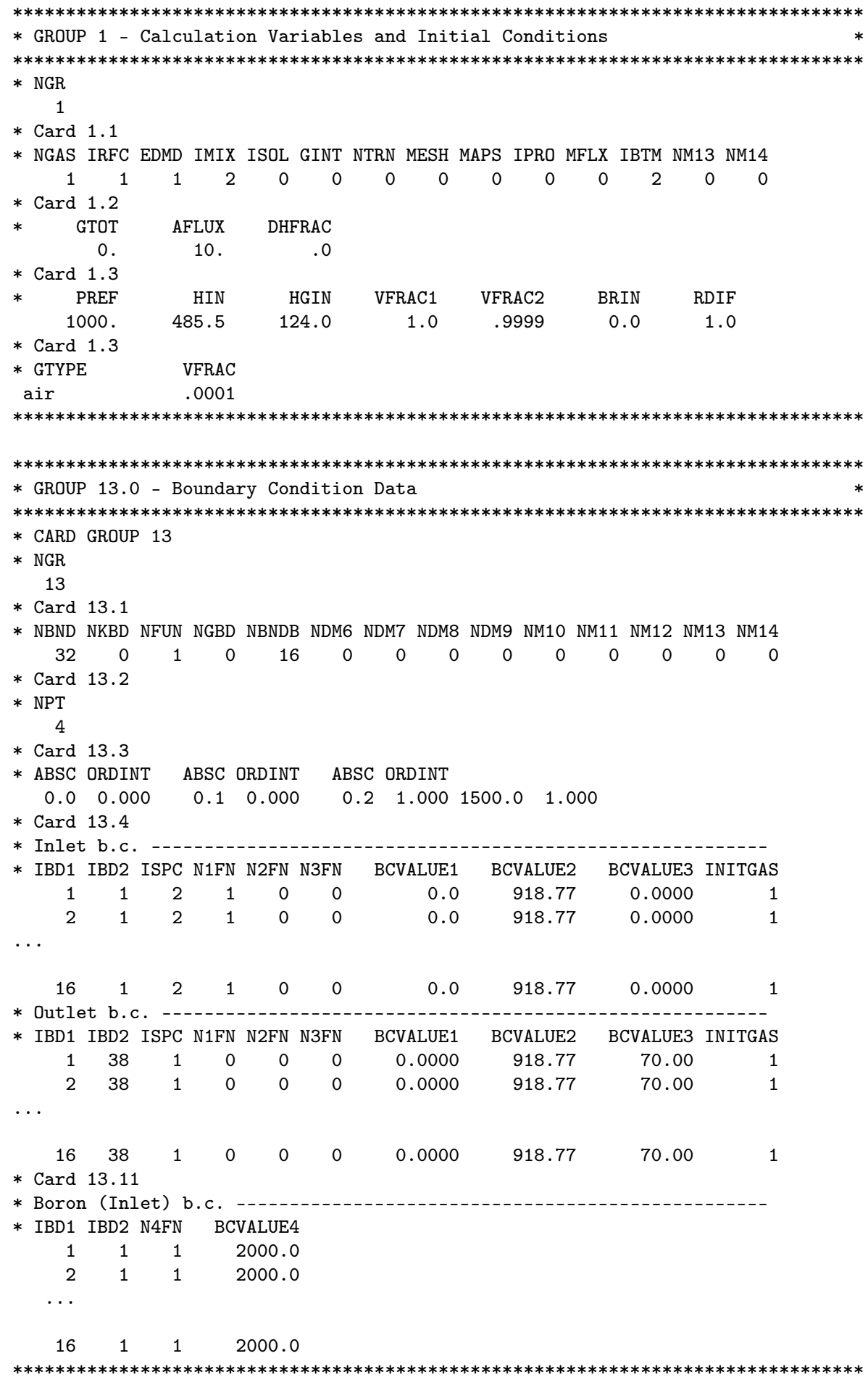




\subsection{Turbulent Mixing and Void Drift Modeling}

CARD GROUP 12 provides input for the turbulent mixing and void drift model as specified by IMIX on CARD 1.1. This input is optional and should be used only for problems where is a significant effect of these phenomena on the behavior of the system.

In the subchannel codes, the exchange of momentum, energy, and mass due to turbulence, or the so-called turbulent diffusion or turbulent mixing, is commonly modeled in analogy to the molecular diffusion under the assumption of a linear dependence between the exchange rate of the given quantity and its gradient in the medium. The proportionality coefficients are called turbulent diffusion coefficients. Unlike molecular diffusion coefficients, which are material dependent, the turbulent diffusion coefficients depend only on the location in the flow domain. The turbulent kinematical viscosity and turbulent temperature diffusivity are in the same order of magnitude (turbulent Prandtl number approaches unity). The latter allows applying the same turbulent diffusion coefficient to all momentum, mass, and energy exchanges: $C_{t}=D_{t}=v_{t}=a_{t}$. In the case of a gradient in the $y$ direction, the aforementioned assumption takes the form of:

Turbulent mixing of mass:

$$
\dot{m}_{k}=-\rho D_{t} \frac{d c}{d y} A=-C_{t} \frac{d\left(\alpha_{k} \rho_{k}\right)}{d y} A
$$

Turbulent mixing of momentum:

$$
\dot{I}_{k}=-\rho \nu_{t} \frac{d U}{d y} A=-C_{t} \frac{d\left(\alpha_{k} \rho_{k} U_{k}\right)}{d y} A=-C_{t} \frac{d G_{k}}{d y} A
$$

Turbulent mixing of energy:

$$
\dot{Q}_{k}=-\rho c_{p} \alpha_{t} \frac{d T}{d y} A=-C_{t} \frac{d\left(\alpha_{k} \rho_{k} c_{p, k} T_{k}\right)}{d y} A=-C_{t} \frac{d\left(\alpha_{k} \rho_{k} h_{k}\right)}{d y} A
$$

Here, the index $k$ stands for the given field (liquid, vapor, and droplets); $D_{t}$ is the turbulent diffusion coefficient for mass transfer; $\nu_{t}$ is the turbulent kinematical viscosity; $\alpha_{t}$ is the turbulent temperature conductivity; $C$ is the concentration; $c_{p}$ is the specific heat capacity; $A$ is the area relevant for lateral exchange; $\alpha, \rho, U$, and $h$ are, respectively, the volume fraction of given field, density, vertical velocity, and enthalpy.

In the subchannel analyses, very often the ratio $C_{t} / d y$ is substituted with the ratio of the turbulent kinematic viscosity $\epsilon$ and the mixing length $l, \epsilon / l$, and the mixing length is commonly approximated as the centroid distance between the adjacent subchannels. Regarding turbulent diffusion coefficients, a dimensionless parameter can be defined:

$$
\beta=\frac{C_{t}}{\Delta y \bar{U}}
$$

where $\bar{U}$ is the area-averaged veritical velocity of the adjacent subchannels and is defined as: 


$$
\bar{U}=\frac{A_{i} U_{i}+A_{j} U_{j}}{A_{i}+A_{j}}
$$

Using the definition of the turbulent mixing coefficient, the exchange rate of mass, momentum and energy (Equations $-35-$ through $-37-$ ) can be written as:

Turbulent mixing of mass:

$$
\dot{m}_{k}=-\beta \frac{\bar{G}}{\rho} \Delta\left(\alpha_{k} \rho_{k}\right) A
$$

Turbulent mixing of momentum:

$$
\dot{I}_{k}=-\beta \frac{\bar{G}}{\rho} \Delta G_{k} A
$$

Turbulent mixing of energy:

$$
\dot{Q}_{k}=-\beta \frac{\bar{G}}{\rho} \Delta\left(\alpha_{k} \rho_{k} h_{k}\right) A
$$

where:

$$
\bar{G}=\frac{A_{i} G_{t o t, i}+A_{j} G_{t o t, j}}{A_{i}+A_{j}}
$$

As concluded from Equation -39-, the turbulent mixing coefficient is a function of the particular geometry and the flow conditions. Under single-phase flow conditions, it is usually correlated to the flow Reynolds number, subchannel hydraulic diameter, heated rod diameter, gap width, and the centroid between the adjacent subchannels: $\beta_{S P}=f\left(R e, d_{\text {hyd }}, d_{\text {gap }}, \operatorname{drod}, \Delta Y\right)$.

It is experimentally observed that in a two-phase flow the turbulent mixing is much higher than in a singlephase flow. Most often, the dependence of the mixing rate on the flow regime is modeled by the Beus correlation -(Beus, S.G., 1970). The two-phase turbulent velocity is assumed to be a function of the singlephase turbulent velocity: $\left(\frac{\epsilon}{l}\right)_{T P}=\theta_{T P}\left(\frac{\epsilon}{l}\right)_{S P}$, where the "two-phase multiplier", $\theta_{T P}$, depends on the quality. The approach by Faya (Faya, A.J.G., 1979) has been adopted in the subchannel analysis codes. Faya has slightly modified the Beus approach by applying the two-phase multiplier $\theta_{T P}$ to the single-phase mixing coefficient:

$$
\beta_{T P}=\theta_{T P} \beta_{S P}
$$

where $\theta_{T P}=f(x)$.

The mixing rate, and hence the turbulent velocity, reaches its maximum at the slug-annular regime transition point. According to the model of Wallis (Wallis, G.B., 1969), this point can be obtained by an expression for the corresponding quality: 


$$
x_{\text {max }}=\frac{0.6+\frac{0.4 \sqrt{g \rho_{l i q}\left(\rho_{l i q}-\rho_{v a p}\right) d_{h y d}}}{G_{t o t}}}{\sqrt{\frac{\rho_{l i q}}{\rho_{\text {vap }}}}+0.6}
$$

The function for $\theta_{T P}$ is assumed to increase linearly for $x \leq x_{\max }$ and to decrease hyperbolically for $x>x_{\max }$.

$$
\theta_{T P}= \begin{cases}1+\left(\theta_{\max }-1\right) \frac{x}{x_{\max }}, & \text { if } x \leq x_{\max } \\ 1+\left(\theta_{\max }-1\right) \frac{x_{\max }-x_{0}}{x-x_{0}}, & \text { if } x>x_{\max }\end{cases}
$$

where:

$$
R e=\frac{G_{t o t} d_{h y d}}{\mu_{m i x}}
$$

and:

$$
\mu_{m i x}=\left(1-\alpha_{v a p}\right) \mu_{l i q}+\alpha_{v a p} \mu v a p
$$

The parameter $\theta_{\max }$, which is the maximum of the ratio $\frac{\beta T P}{\text { betaSP }}$, is treated as a constant and can be estimated experimentally.

In the COBRA/TRAC code version, only a single-phase mixing coefficient (single-phase liquid for void fractions below a value of $\mathbf{0 . 6}$, single-phase vapor for void fractions above a value of $\mathbf{0 . 8}$, and a ramp between the two) has been modeled by means of the traditional mixing coefficient approach. Later, in the FLECHT SEASET code version, a void drift model based on the work of Lahey and Kelly has been employed. Void drift was only assumed to occur when the liquid is continuous phase and its modeling has been not applied to the hot wall flow regimes.

The current turbulent mixing and void drift models assume that the net two-phase mixing (including void drift) is proportional to the non-equilibrium void fraction gradient. At an annular film flow regime a void drift offset is assumed and only the turbulent mixing of vapor and entrained droplets is modeled. In other words, the void drift is only modeled in bubbly, slug, and churn flow, where liquid is the continuous phase and vapor is the dispersed phase.

The lateral exchange due to turbulent mixing is modeled as follows:

Turbulent mixing of mass in phase $k$ :

$$
\dot{m}_{k}^{T M}=-\beta \frac{\bar{G}}{\rho} \Delta\left(\alpha_{k} \rho_{k}\right) A
$$

Turbulent mixing of momentum in phase $k$ :

$$
\dot{I}_{k}^{T M}=-\beta \frac{\bar{G}}{\bar{\rho}} \Delta G_{k} A
$$


Turbulent mixing of energy in phase $k$ :

$$
\dot{Q}_{k}^{T M}=-\beta \frac{\bar{G}}{\rho} \Delta\left(\alpha_{k} \rho_{k} h_{k}\right) A
$$

In Equations $-46-$ through $-48-, \beta=\theta_{T P} \beta_{S P}$ is the two-phase turbulent mixing coefficient.

Currently the single phase mixing coefficient $\beta_{S P}$ may be either specified as a single input value or internally calculated with an empirical correlation by Rogers and Rosehart (Rogers, J.T.andRosehart, R.G., 1972):

$$
\beta_{S P}=\frac{1}{2} 0.0058\left(\frac{d_{\text {gap }}}{d_{\text {rod }}}\right)^{-1.46} R e^{-0.1}\left[1+\left(\frac{d_{\text {hyd,j }}}{d_{\text {hyd,i }}}\right)\right]^{1.5} \frac{d_{\text {hyd,i }}}{d_{\text {rod }}}
$$

The two-phase multiplier $\beta_{T P}$ is calculated using the Beus approach for two-phase turbulent mixing.

The lateral exchange due to void drift is modeled as follows:

Mass exchange in phase $k$ by void drift:

$$
\dot{m}_{k}^{V D}=-\beta \frac{\bar{G}}{\rho}\left(\alpha_{k, j, E Q} \rho_{k, j, E Q}-\alpha_{k, i, E Q} \rho_{k, i, E Q}\right) A
$$

Momentum exchange in phase $k$ by void drift:

$$
\dot{I}_{k}^{V D}=-\beta \frac{\bar{G}}{\bar{\rho}}\left(G_{k, j, E Q}-G_{k, i, E Q}\right) A
$$

Energy exchange in phase $k$ by void drift:

$$
\dot{Q}_{k}^{V D}=-\beta \frac{\bar{G}}{\rho}\left(\alpha_{k, j, E Q} \rho_{k, j, E Q} h_{k, j, E Q}-\alpha_{k, i, E Q} \rho_{k, i, E Q} h_{k, i, E Q}\right) A
$$

According to Levy (Levy, S., 1963) the equilibrium density distribution is related to the mass flux distribution by the expression:

$$
\rho_{m i x, E Q}=\alpha_{l i q} \rho_{l i q}+\alpha_{v a p} \rho_{v a p}=\alpha G_{t o t, E Q}+b
$$

For phase $k$ this yields:

$$
\left(\alpha_{k, j, E Q} \rho_{k, j, E Q}-\alpha_{k, i, E Q} \rho_{k, i, E Q}\right)=f_{s i g n} \frac{\alpha_{v a p} \rho_{k}}{\bar{G}_{t o t}}\left(G_{t o t, j, E Q}-G_{t o t, i, E Q}\right)
$$

where: 


$$
f_{\text {sign }}= \begin{cases}1 & \text { for vapor } \\ -1 & \text { for liquid }\end{cases}
$$

Other parameters are:

$$
\begin{gathered}
\bar{G}_{t o t}=\frac{A_{i} G_{t o t, i}+A_{j} G_{t o t, j}}{A_{i}+A_{j}} \\
\alpha_{v a \bar{p}} \rho_{v a p}=\frac{A_{i} \alpha_{v a p, i} \rho_{v a p, i}+A_{j} \alpha_{v a p, j} \rho_{v a p, j}}{A_{i}+A_{j}} \\
\alpha_{v a \bar{p}} \rho_{l i q}=\frac{A_{i} \alpha_{v a p, i} \rho_{l i q, i}+A_{j} \alpha_{v a p, j} \rho_{l i q, j}}{A_{i}+A_{j}}
\end{gathered}
$$

The equilibrium vapor phase distribution is given by:

$$
\left(\alpha_{v a p, j, E Q} \rho_{v a p, j, E Q}-\alpha_{v a p, i, E Q} \rho_{v a p, i, E Q}\right)=K_{M} \frac{G_{t o t, j}-G_{t o t, i}}{G_{t o t, j}+G_{t o t, i}}\left(\alpha_{v a p, i} \rho_{v a p, i}+\alpha_{v a p, j} \rho_{v a p, j}\right)
$$

and the equilibrium liquid phase distribution is given by:

$$
\left(\alpha_{l i q, j, E Q} \rho_{l i q, j, E Q}-\alpha_{l i q, i, E Q} \rho_{l i q, i, E Q}\right)=K_{M} \frac{G_{t o t, j}-G_{t o t, i}}{G_{t o t, j}+G_{t o t, i}}\left(\alpha_{v a p, i} \rho_{l i q, i}+\alpha_{v a p, j} \rho_{l i q, j}\right)
$$

The corresponding equilibrium momentum flux distribution for the vapor phase becomes:

$\left(G_{v a p, j, E Q}-G_{v a p, i, E Q}\right)=K_{M} \frac{G_{t o t, j}-G_{t o t, i}}{G_{t o t, j}+G_{t o t, i}}\left(\alpha_{v a p, i} \rho_{v a p, i} U_{v a p, i}+\alpha_{v a p, j} \rho_{v a p, j} U_{v a p, j}\right)=K_{M} \frac{G_{t o t, j}-G_{t o t, i}}{G_{t o t, j}+G_{t o t, i}}\left(G_{v a p, i}+G_{v a p, j}\right)$ and for the liquid phase:

$\left(G_{l i q, j, E Q}-G_{l i q, i, E Q}\right)=K_{M} \frac{G_{t o t, j}-G_{t o t, i}}{G_{t o t, j}+G_{t o t, i}}\left(\alpha_{v a p, i} \rho_{l i q, i} U_{l i q, i}+\alpha_{v a p, j} \rho_{l i q, j} U_{l i q, j}\right)=K_{M} \frac{G_{t o t, j}-G_{t o t, i}}{G_{t o t, j}+G_{t o t, i}}\left(G_{l i q, i}+G_{l i q, j}\right)$

The corresponding equilibrium energy distribution for the vapor phase becomes:

$\left(\alpha_{v a p, j, E Q} \rho_{v a p, j, E Q} h_{v a p, j, E Q}-\alpha_{v a p, i, E Q} \rho_{v a p, i, E Q} h_{v a p, i, E Q}\right)=K_{M} \frac{G_{t o t, j}-G_{t o t, i}}{G_{t o t, j}+G_{t o t, i}}\left(\alpha_{v a p, i} \rho_{v a p, i} h_{v a p, i}+\alpha_{v a p, j} \rho_{v a p, j} h_{v a p, j}\right)$ 
and for the liquid phase:

$$
\left(\alpha_{l i q, j, E Q} \rho_{l i q, j, E Q} h_{l i q, j, E Q}-\alpha_{l i q, i, E Q} \rho_{l i q, i, E Q} h_{l i q, i, E Q}\right)=K_{M} \frac{G_{t o t, j}-G_{t o t, i}}{G_{t o t, j}+G_{t o t, i}}\left(\alpha_{l i q, i} \rho_{l i q, i} h_{l i q, i}+\alpha_{l i q, j} \rho_{l i q, j} h_{l i q, j}\right)
$$

\subsubsection{Instructions to CARD GROUP 12}

The choice of the turbulent mixing and void drift modeling is controlled by the parameter IMIX specified on CARD 1.1. If IMIX is set to zero, neither turbulent mixing nor void drift are modeled and CARD GROUP 12 is omitted.

If IMIX is set to one (1), a constant mixing coefficient BETA (used at both single- and two-phase conditions) and the equilibrium distribution weighting factor AAAK $\left(K_{M}\right.$ in the above equations) are specified on CARD 12.1. A suggested value of AAAK is 1.4 according to Kelly, et al. (1981). An example is given below.

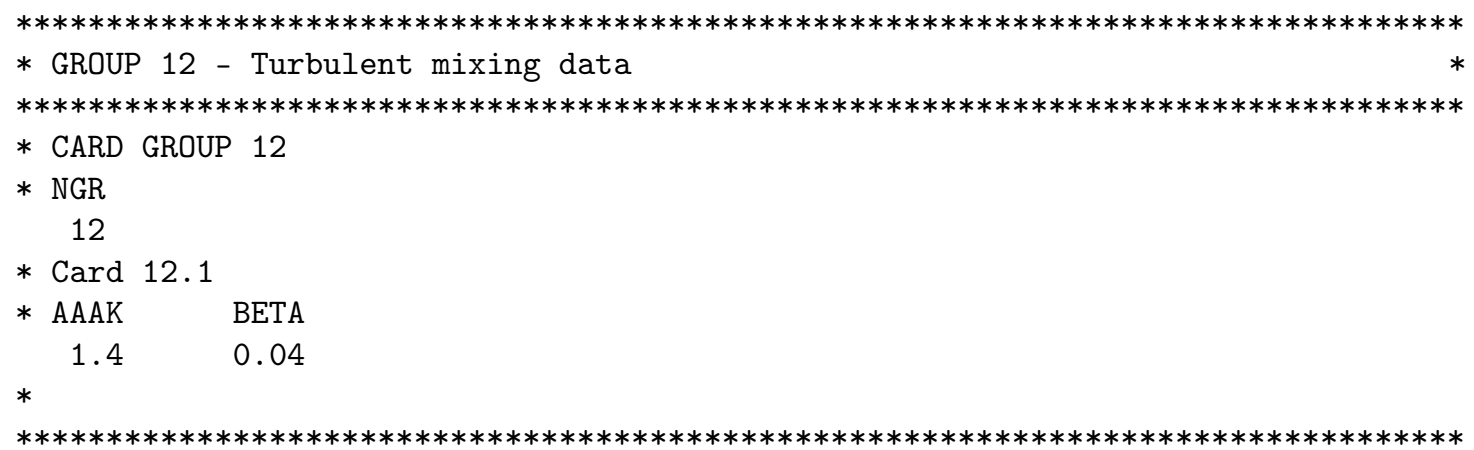

If IMIX is set to two (2), the single-phase mixing coefficient will be calculated with the correlation by Rogers and Rosehart and the two-phase multiplier will be calculated using the Beus approach. The input on CARD 12.2 includes the equilibrium distribution weighting factor AAAK; the outside rod diameter DFROD $\left(d_{\text {rod }}\right.$ in Equation -36-); and the ratio between the maximum two-phase turbulent mixing and the single-phase liquid mixing THETM $\left(\theta_{\max }\right.$ in Equations $-33-$ and $\left.-34-\right)$. The value of the outside rod diameter, DFROD, should be consistent with DROD read on CARD 9.2 or CARD 9.6. A suggested value of THETM is 5.0 according to Sato (1992). An example is given below. 


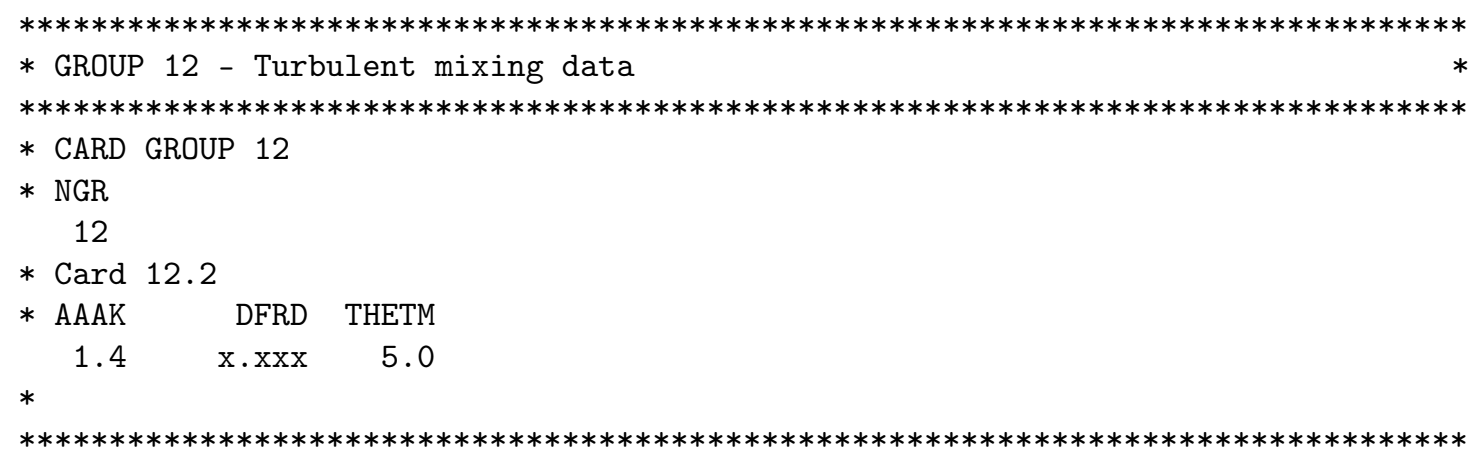

\subsubsection{Instructions to CARD GROUP 12}

The choice of the turbulent mixing and void drift modeling is controlled by the parameter IMIX specified on CARD 1.1. If IMIX is set to zero, neither turbulent mixing nor void drift are modeled and CARD GROUP 12 is omitted.

If IMIX is set to one (1), a constant mixing coefficient BETA (used at both single- and two-phase conditions) and the equilibrium distribution weighting factor AAAK $\left(K_{M}\right.$ in the above equations) are specified on CARD 12.1. A suggested value of AAAK is 1.4 according to Kelly, et al. (1981). An example is given below.

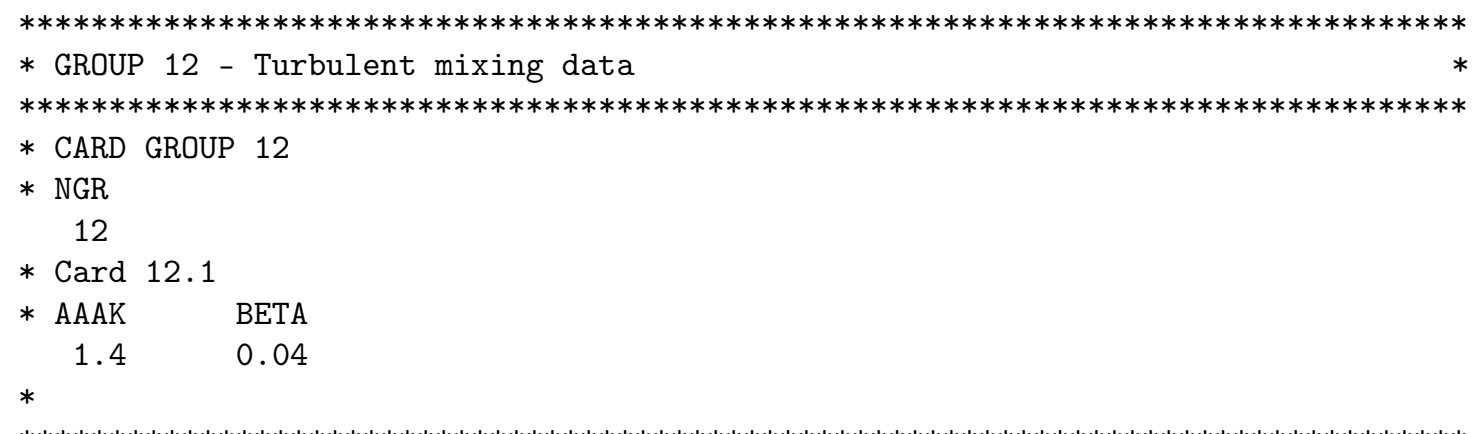

If IMIX is set to two (2), the single-phase mixing coefficient will be calculated with the correlation by Rogers and Rosehart and the two-phase multiplier will be calculated using the Beus approach. The input on CARD 12.2 includes the equilibrium distribution weighting factor AAAK; the outside rod diameter DFROD $\left(d_{\text {rod }}\right.$ in Equation -36-); and the ratio between the maximum two-phase turbulent mixing and the single-phase liquid mixing THETM $\left(\theta_{\max }\right.$ in Equations $-33-$ and $\left.-34-\right)$. The value of the outside rod diameter, DFROD, should be consistent with DROD read on CARD 9.2 or CARD 9.6. A suggested value of THETM is 5.0 according to Sato (1992). An example is given below. 


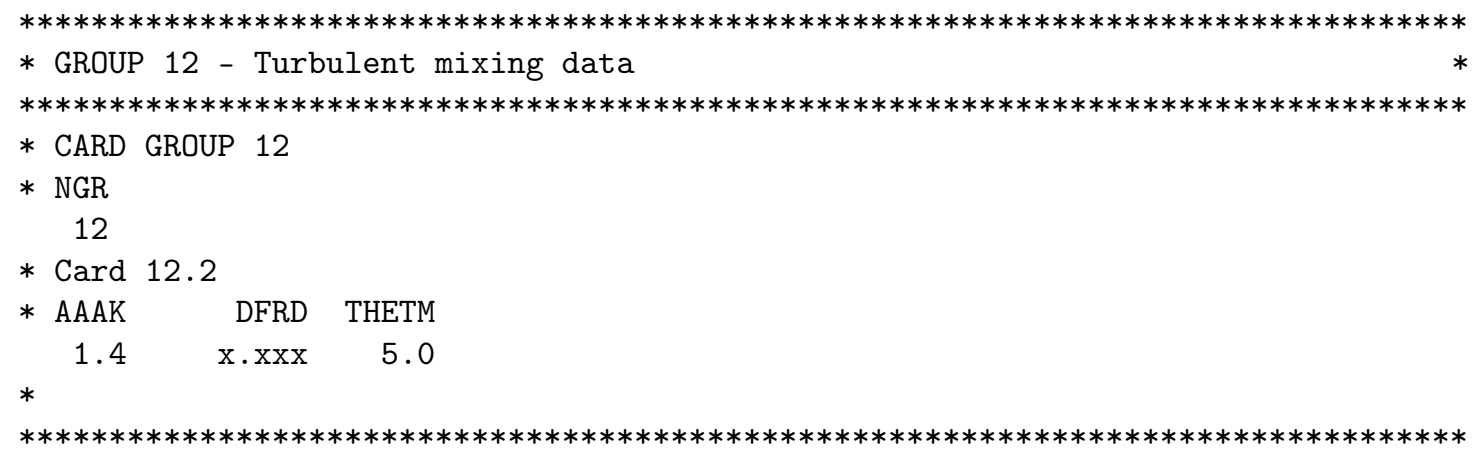

\subsection{Results Reporting}

The output information to be printed out can be selected by input on the legacy CARD GROUP 14. In total, ten output files can be generated. A short description of the CTF output files (produced by legacy Card Group 14) is provided in Table 12. Guidance to CARD GROUP 14 is given in the following section.

Table 12: List of Generated Output Files

\begin{tabular}{|l|l|c|c|}
\hline File Name & Description & SI Units & US Units \\
\hline deck.cdm & $\begin{array}{l}\text { A dump file for restarting the calculation; generated } \\
\text { if DUMPF = 1 in CARD INPUT .2; empty if DUMPF } 0\end{array}$ & $\mathrm{X}$ & $\mathrm{X}$ \\
\hline deck.crs & $\begin{array}{l}\text { An empty restart file; it must be overwritten by deck.cdm } \\
\text { before a restart calculation is executed }\end{array}$ & $\mathrm{X}$ & $\mathrm{X}$ \\
\hline deck.out & $\begin{array}{l}\text { Input repitition, geometry information } \\
\text { Subchannel, gap, rod, and unheated conductor results }\end{array}$ & - & $\mathrm{X}$ \\
\hline deck.run & Convergence history & - & $\mathrm{X}$ \\
\hline dnb.out & DNB information & $\mathrm{X}$ & $\mathrm{X}$ \\
\hline heat.time & Global transient heat balance & - & - \\
\hline krysolv.out & $\begin{array}{l}\text { Debug output for Krylov solver convergence. Generated if } \\
\text { the flag IOPT read on CARD 14.1 is set to 2. }\end{array}$ & $\mathrm{X}$ & $\mathrm{X}$ \\
\hline massflow.time & Global transient mass balance & $\mathrm{X}$ & - \\
\hline mixture_temp.out & $\begin{array}{l}\text { Average coolant temperature in node (i,j) (simplified output } \\
\text { needed for validation purposes). Generated if the flag IOPT } \\
\text { read on CARD 14.1 is set to 3. }\end{array}$ & $\mathrm{X}$ & - \\
\hline results_channel.out & Subchannel results & $\mathrm{X}$ & - \\
\hline results_gap.out & Gap results & - & - \\
\hline time.out & $\begin{array}{l}\text { Debug output for inner/outer iterations information. } \\
\text { Generated if the flag IOPT read on CARD 14.1 is set to 2. }\end{array}$ & $\begin{array}{l}\text { - } \\
\text { Subchannel void fraction (simplified output needed for } \\
\text { CARD 14.1 is set to 4. }\end{array}$ & - \\
\hline void.out & & - & \\
\hline
\end{tabular}




\subsubsection{Instructions to CARD GROUP 14 (Legacy)}

The user can request a complete printout of the calculated results by setting the parameter N1 on CARD 14.1 equal to 5 and the parameters NOUT1, NOUT2, NOUT3 and NOUT4 equal to zero. In this case results for all subchannels, gaps, heated and unheated conductors will be printed out in the corresponding output files. If the user needs output information for selected subchannels, gaps, or conductors only, that can be specified by different combinations of values for N1, NOUT1, NOUT2, NOUT3 and NOUT4 and additional input on CARDS 4.2 through 4.5 .

The property table will be printed in the deck.out file if the parameter IPROPP on CARD 4.1 is set to 1 . The parameter IOPT controls the printout of additional debug information.

For the example given below, the output will contain results for subchannels 5, 13, 17, and 20; for gaps 9, 25, and 36; for heated conductors 4, 10, and 12; and for unheated conductors 5 and 8 . In addition, the subchannel void fractions (for all subchannels) will be provided in the output file void.out.

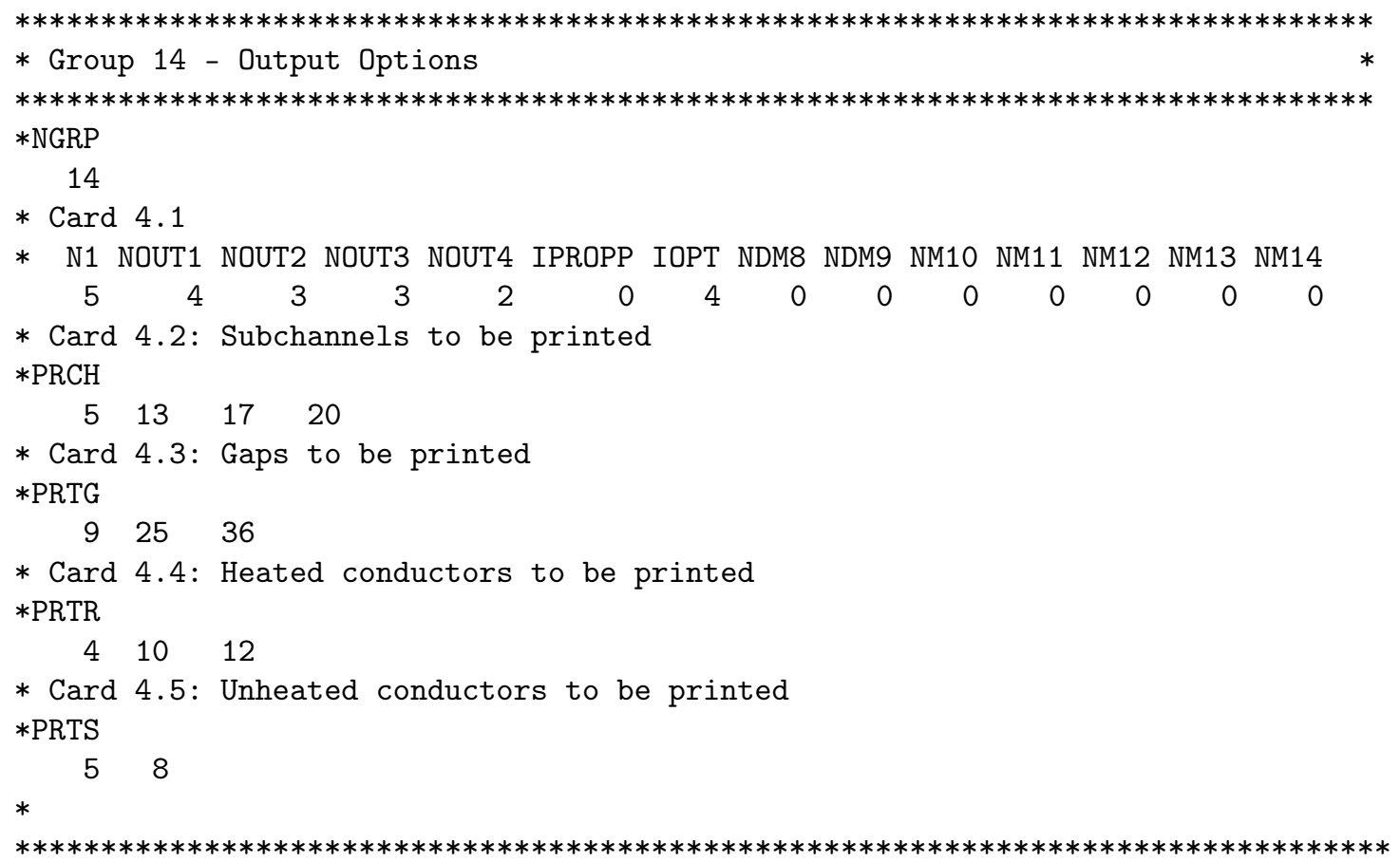

\subsection{Main Problem Control and Time Domain Data}

A brief description of the required input for the main problem control data and time domain data is provided in the following sub-sections. 


\subsubsection{Instructions to Input of Main Problem Control Data}

The main problem control data is read on CARDS INPUT 1 through 3 and CARD COBRA 1 . CARD INPUT 1 selects the units in input and output files (SI or US). CARD INPUT 2 specifies if an initial or restart calculation will be performed and whether a restart file, deck.cdm, will be generated. The inner and outer iterations limits are read on CARD INPUT 4. CARD COBRA 1 gives alphanumeric information to identify the simulation.

In the example of input for main problem control data given below, the input deck will be written in US units, but the output decks will be in SI units (ICOBRA $=0$ ); it will be an initial run $($ INITIAL $=1)$ and a restart file will not be generated (DUMPF $=0$ ); the outer iteration convergence criterion (EPSO) is set to $10^{-3}$; the maximum number of outer iterations (OITMAX) is equal to 5 ; and the maximum number of inner iterations (IITMAX) is equal to 40 .

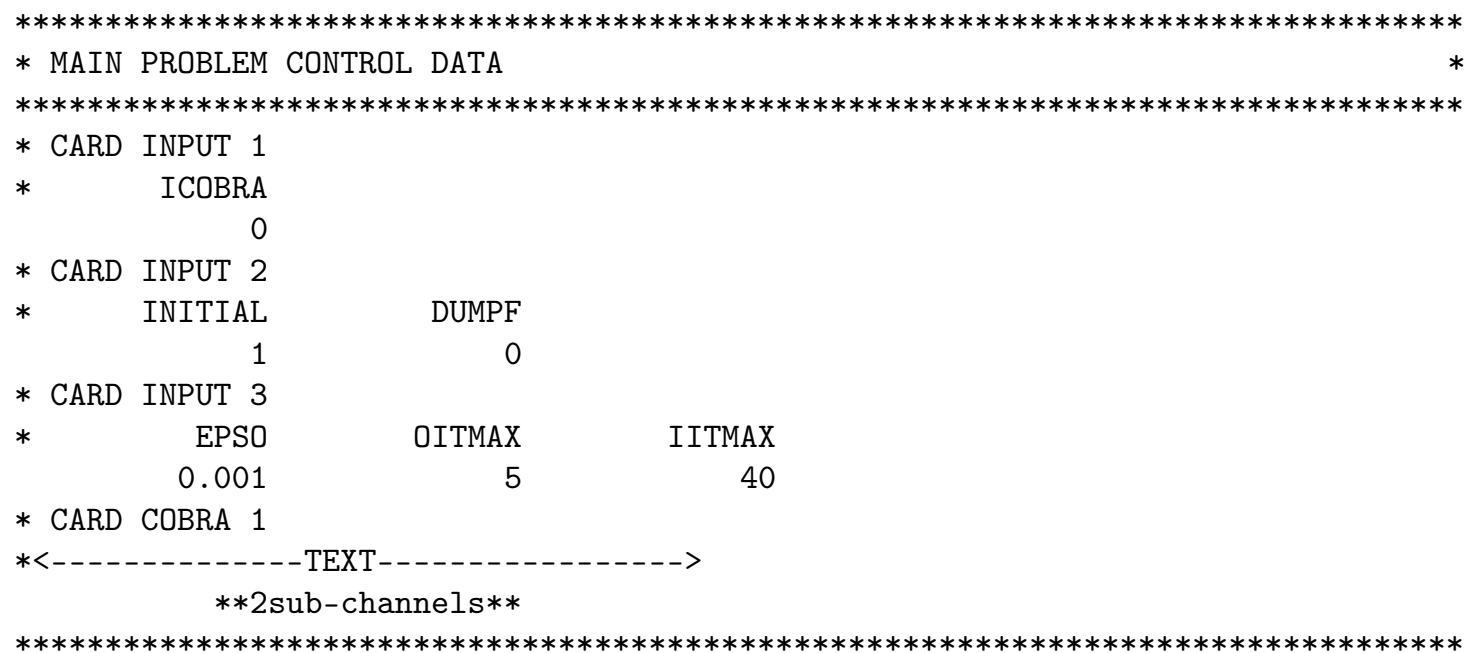

\subsubsection{Instructions to Input of Time Domain Data}

After all component data have been entered, the user must define the time domain for the simulation. The total time can be divided into several domains of specified duration. Each time domain can have different minimum and maximum time step sizes and different edit intervals as entered in Card 15.1. The card must be repeated for each time domain desired. A final time domain with a negative value for DTMIN must be entered to terminate the calculation.

In the example below, the whole calculation of 12 seconds is divided into two time domains. The first time domain has a duration of 2 seconds (TEND $=2$ ), a minimum time step size of $10^{-6}$ seconds (DTMIN $=10^{-6}$ ) and a maximum time step size of $10^{-3}$ seconds, (DTMAX $=10^{-3}$ ). The results will be printed out every half second $($ EDINT $=0.5)$ and the dump file deck.cdm will be written every second. The second time domain has a duration of 10 seconds (TEND $=10$ ), a minimum time step size of $10^{-6}$ seconds, (DTMIN $=10^{-6}$ ), and an increased maximum time step of $10^{-2}$ seconds, (DTMAX $=10^{-2}$ ). The results will be printed out only once at the end $(\operatorname{EDINT}=10.0)$ and the dump file deck.cdm will be written every 5 seconds. 
The parameter RTWFP is a ratio of time step sizes for heat conduction solution and fluid solution. To obtain steady-state conditions, the conduction solution can generally use time steps greater than the fluid solution. For transient calculations RTWFP should be 1.0 .

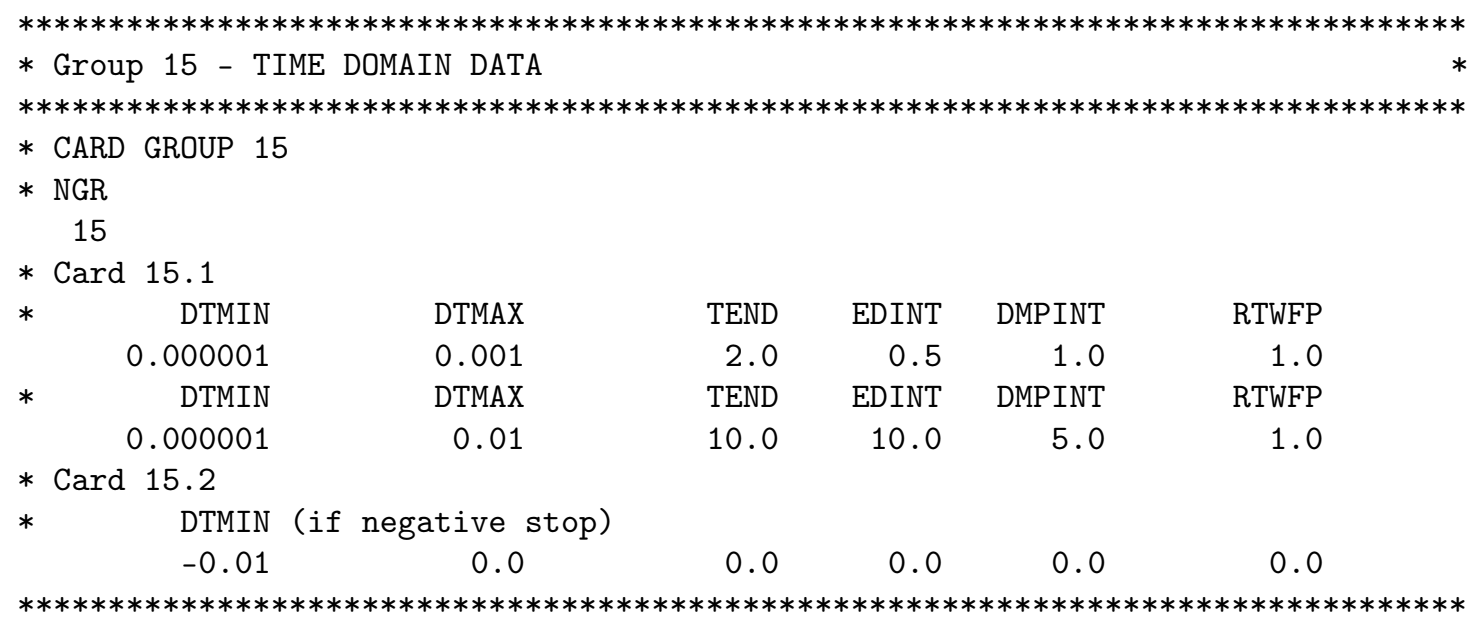

\subsubsection{Instructions to Preparation of Input Files for Restart Calculations}

When the parameter DUMPF read on CARD INPUT 2 is not set to zero, a dump file 'deck.cdm' is generated. In order to restart the calculation, a restart file 'deck.crs' must be read. Because the initial calculation will generate an empty 'deck.crs' file, the dump file 'deck.cdm' must be renamed to 'deck.crs' before restarting the calculation.

Two dump/restart options are possible: "simple" and "full" restart. During the so-called "simple" restart run the user is allowed to change the time domain data, but not the power distribution and the flow conditions. The input file for the "simple" restart must contain CARDS INPUT.1, INPUT.2, INPUT.3, COBRA.1, and CARD GROUP 15; and must not contain CARD GROUP 1 through CARD GROUP 14.

During a "full" restart run, the user can specify changes in the operating conditions, power distribution, boundary conditions, printout options, and the time domain data. The input file for the "full" restart must contain CARDS INPUT.1, INPUT.2, INPUT.3, COBRA.1, and CARD GROUP 15; may contain CARD GROUP 1, CARD GROUP 11, CARD GROUP 12, CARD GROUP 13, and CARD GROUP 14; and must not contain CARD GROUP 2, CARD GROUP 3, CARD GROUP 4, CARD GROUP 5, CARD GROUP 6, CARD GROUP 7, CARD GROUP 8, CARD GROUP 9, or CARD GROUP 10.

Examples for "simple" and "full" restart input files follow.

"Simple" Restart: 


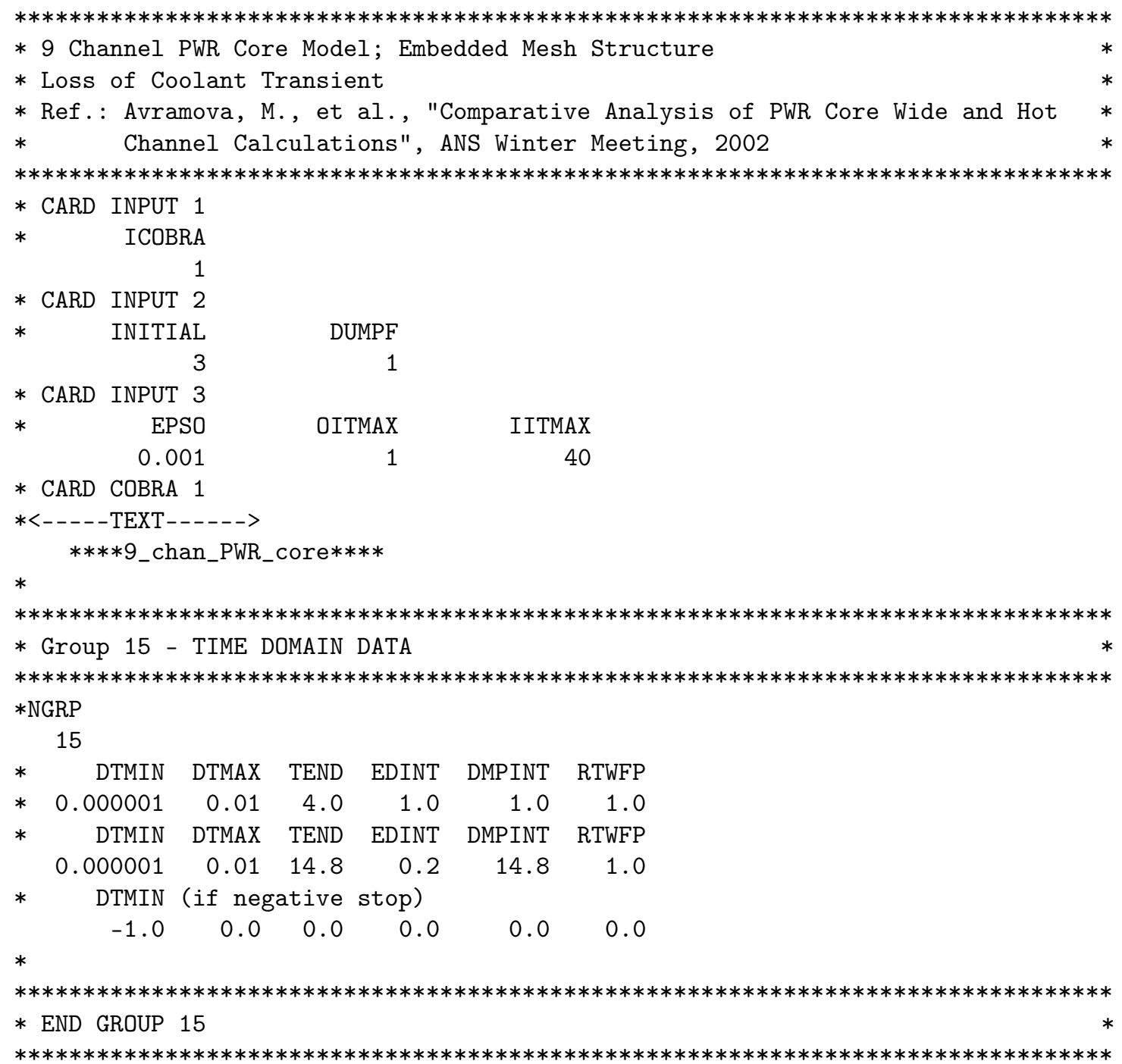

"Full" Restart:

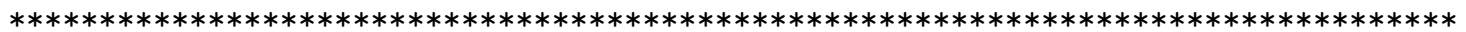

* 9 Channel PWR Core Model; Embedded Mesh Structure

* Loss of Coolant Transient

* Ref.: Avramova, M., et al., "Comparative Analysis of PWR Core Wide and Hot *

* Channel Calculations", ANS Winter Meeting, $2002 \quad *$

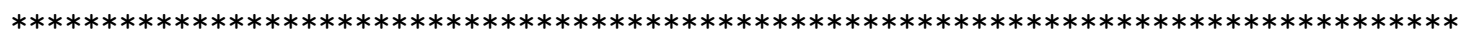

* CARD INPUT 1

* ICOBRA

* CARD INPUT 2

CASL-U-2019-1885-002

207 Consortium for Advanced Simulation of LWRs 


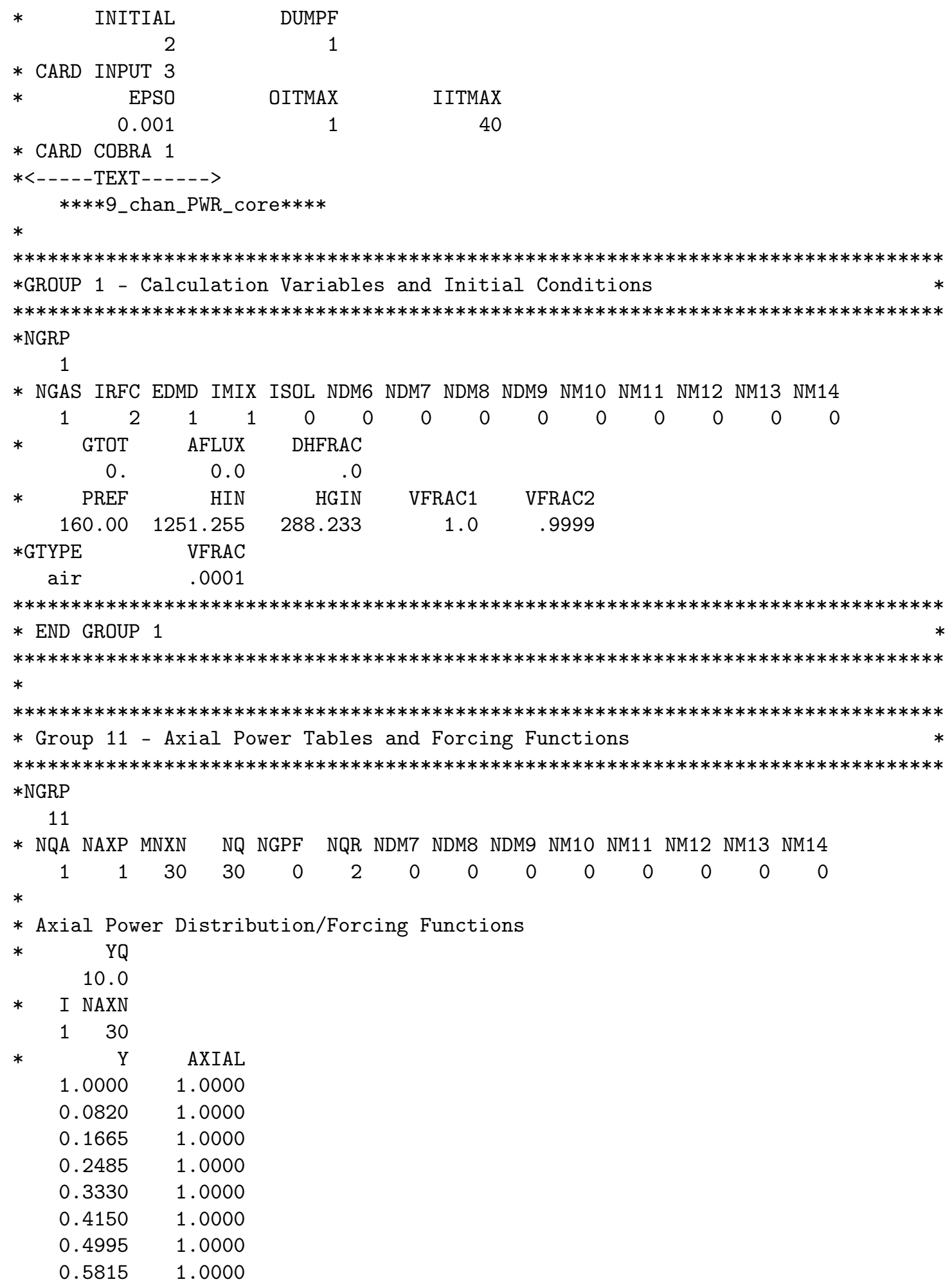




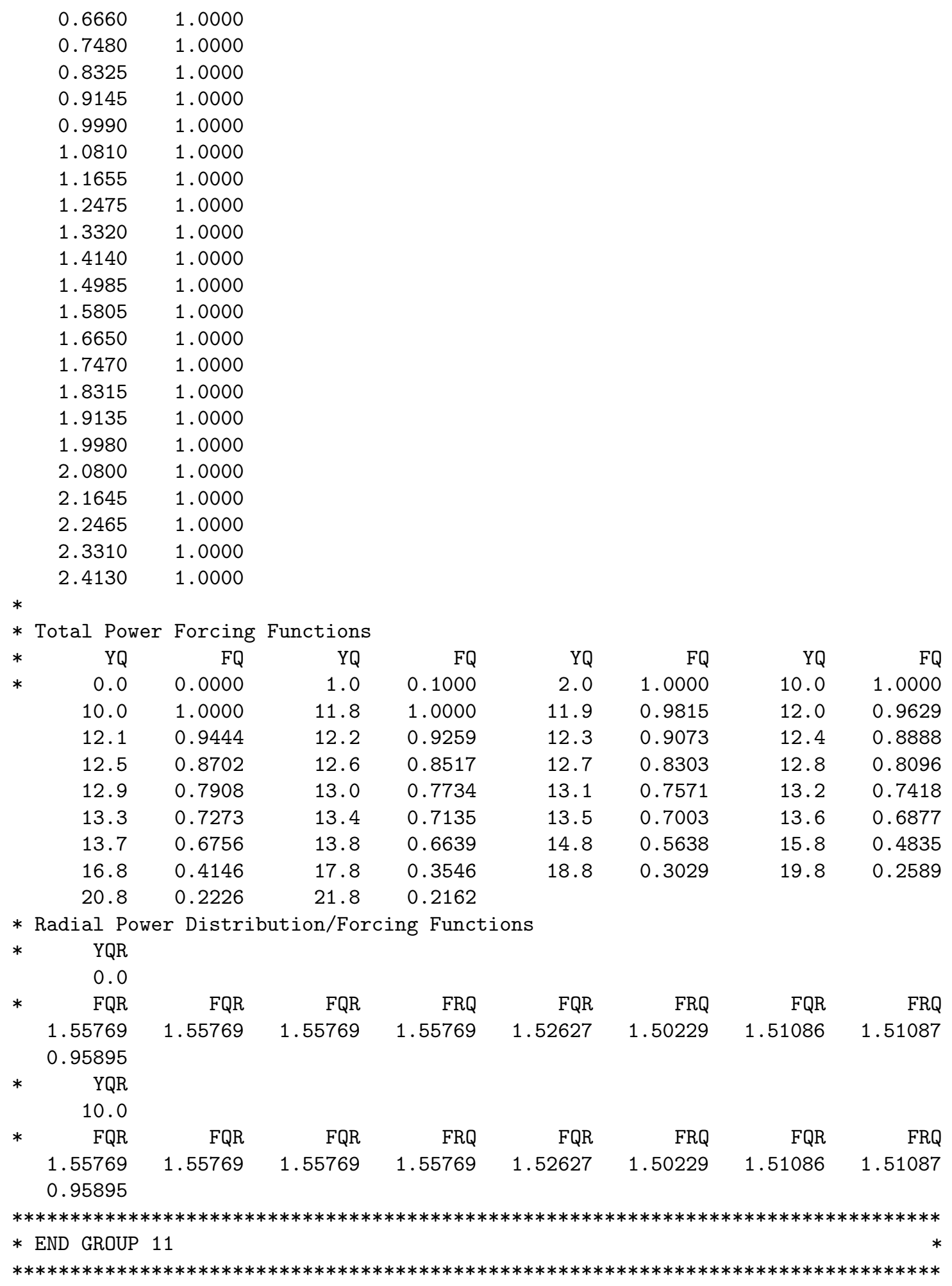




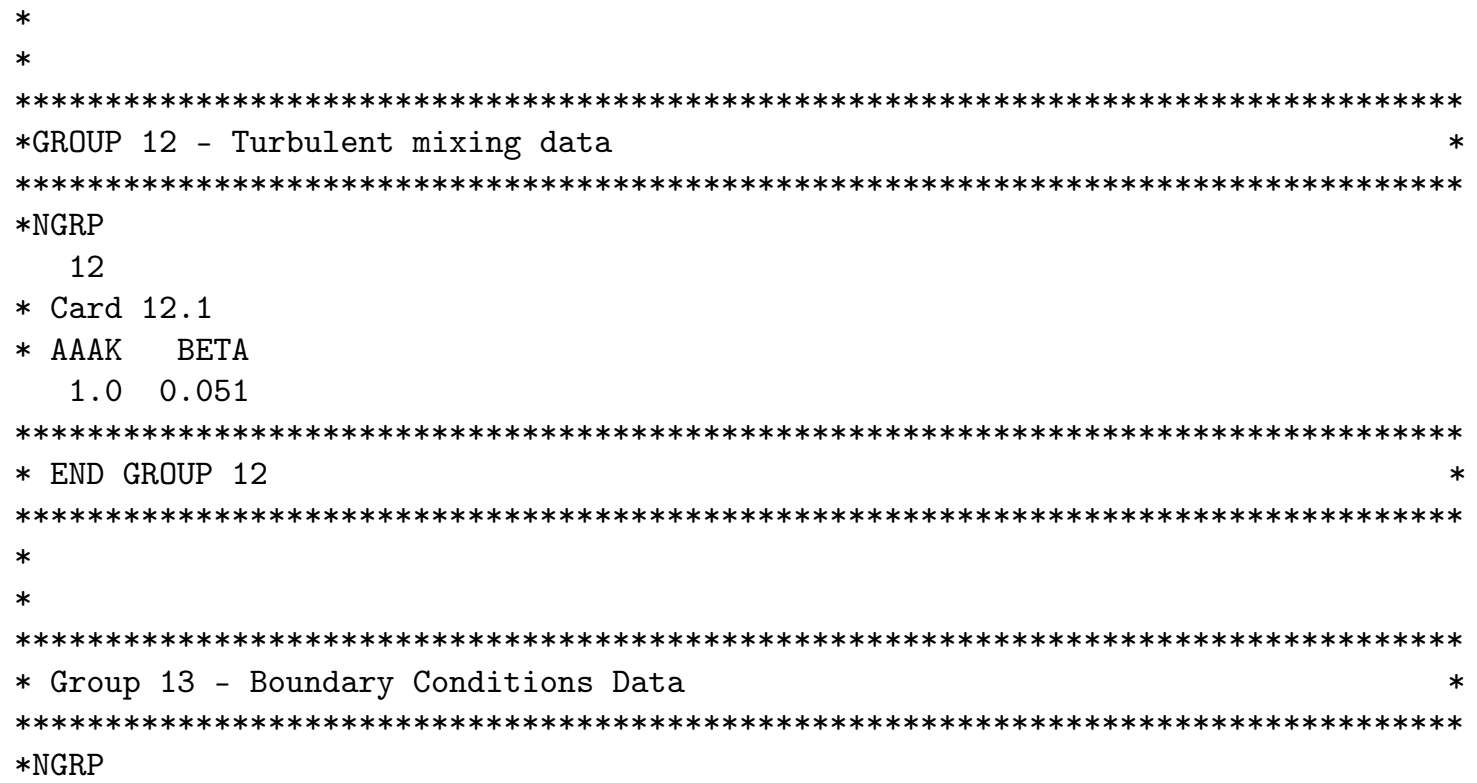

13

*NBND NKBD NFUN NGBD NDM5 NDM6 NDM7 NDM8 NDM9 NM10 NM11 NM12 NM13 NM14

\begin{tabular}{|c|c|c|c|c|c|c|c|c|c|}
\hline 18 & 0 & 0 & 0 & 0 & 0 & 0 & 0 & 0 & 0 \\
\hline \multicolumn{10}{|l|}{ *NPTS } \\
\hline \multirow{2}{*}{\multicolumn{10}{|c|}{32}} \\
\hline & & & & & & & & & \\
\hline *ABSC & ORDINT & ABSC & ORDINT & ABSC & ORDINT & & & & \\
\hline 0.0 & 0.0000 & 0.1 & 1.0000 & 10.0 & 1.0000 & 10.1 & 0.9976 & 10.2 & 0.992 \\
\hline 10.3 & 0.9867 & 10.4 & 0.9795 & 10.5 & 0.9716 & 10.6 & 0.9632 & 10.7 & 0.9547 \\
\hline 10.8 & 0.9459 & 10.9 & 0.9371 & 11.0 & 0.9283 & 11.1 & 0.9196 & 11.2 & 0.91 \\
\hline 11.3 & 0.9023 & 11.4 & 0.8938 & 11.5 & 0.8855 & 11.6 & 0.8773 & 11.7 & 0.86 \\
\hline 11.8 & 0.8615 & 11.9 & 0.8537 & 12.0 & 0.8462 & 13.0 & 0.7817 & 14.0 & 0.73 \\
\hline 15.0 & 0.6960 & 16.0 & 0.6593 & 17.0 & 0.6259 & 18.0 & 0.5953 & 19.0 & 0.5672 \\
\hline 20.0 & 0.5413 & 21.8 & 0.5413 & & & & & & \\
\hline
\end{tabular}

$*$

* Inlet b.c.

*IBD1 IBD2 ISPC N1FN N2FN N3FN BCVALUE1 BCVALUE2 BCVALUE3 INITGAS

$\begin{array}{rrrrrrrrrr}1 & 1 & 2 & 1 & 0 & 0 & .24961 & 1251.255 & 0.0 & 1 \\ 2 & 1 & 2 & 1 & 0 & 0 & .24961 & 1251.255 & 0.0 & 1 \\ 3 & 1 & 2 & 1 & 0 & 0 & .28699 & 1251.255 & 0.0 & 1 \\ 4 & 1 & 2 & 1 & 0 & 0 & .24961 & 1251.255 & 0.0 & 1 \\ 5 & 1 & 2 & 1 & 0 & 0 & 3.1446 & 1251.255 & 0.0 & 1 \\ 6 & 1 & 2 & 1 & 0 & 0 & 9.5544 & 1251.255 & 0.0 & 1 \\ 7 & 1 & 2 & 1 & 0 & 0 & 32.872 & 1251.255 & 0.0 & 1 \\ 8 & 1 & 2 & 1 & 0 & 0 & 75.777 & 1251.255 & 0.0 & 1 \\ 9 & 1 & 2 & 1 & 0 & 0 & 1522.97 & 1251.255 & 0.0 & 1\end{array}$

$*$

* Outlet b.c. -

*IBD1 IBD2 ISPC N1FN N2FN N3FN BCVALUE1 BCVALUE2 BCVALUE3

Consortium for Advanced Simulation of LWRs 210

CASL-U-2019-1885-002 


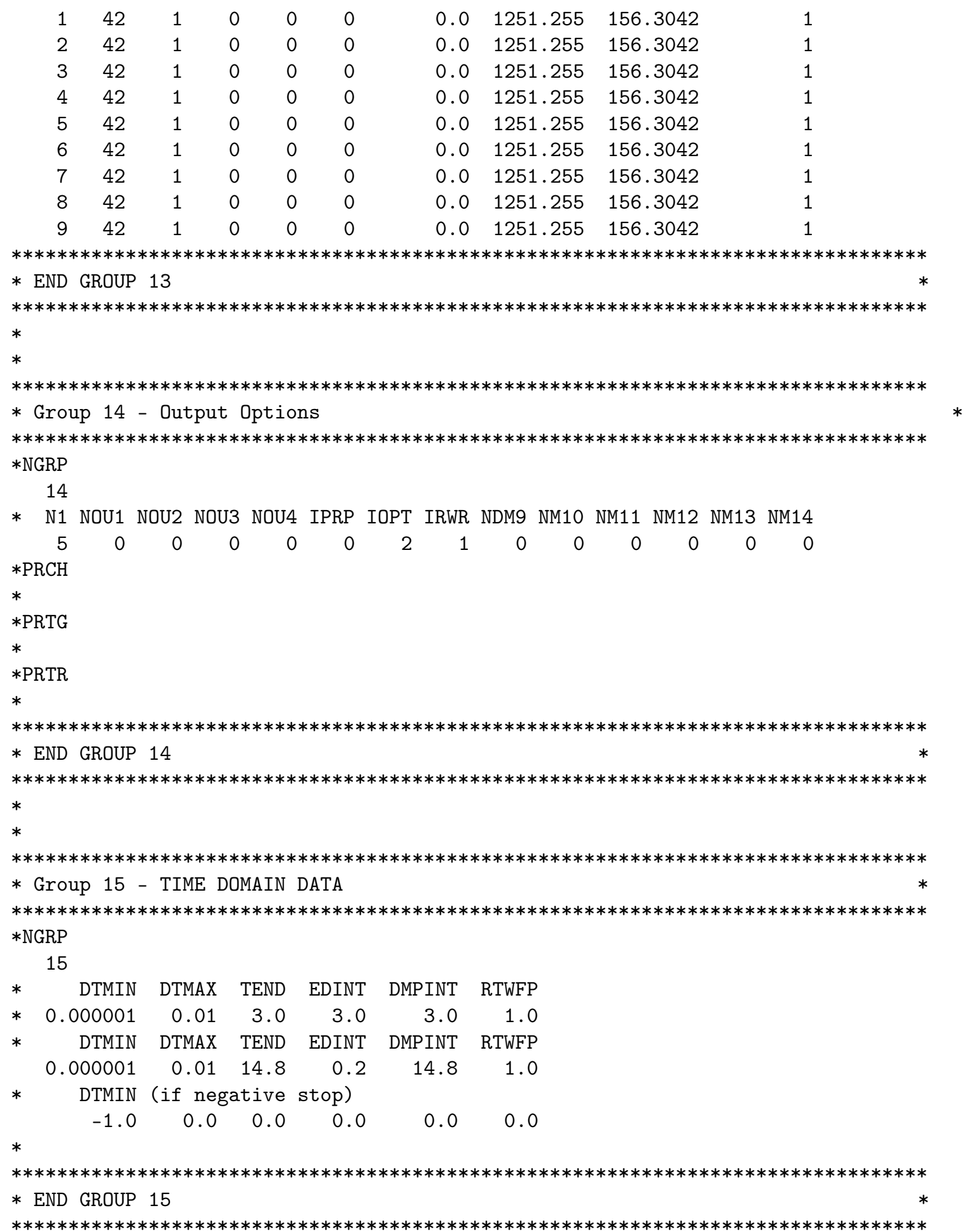




\section{A. Calculation Notes and the 3x3 GE Ex- periments Input Decks}

\section{A.1 GE 3x3 Experimental Parameters}

Table 13: Geometrical characteristics of the GE 3x3 test section

\begin{tabular}{|l|l|}
\hline Parameter & Value \\
\hline Number of Rods & 9 \\
\hline Rod Diameter & $14.7066 \mathrm{~mm}$ \\
\hline Rod-Rod Clearance & $4.2672 \mathrm{~mm}$ \\
\hline Row-Wall Clearance & $3.429 \mathrm{~mm}$ \\
\hline Channel Corner Radius & $10.16 \mathrm{~mm}$ \\
\hline Heated Length & $1828.8 \mathrm{~mm}$ \\
\hline Hydraulic Diameter & $12.0396 \mathrm{~mm}$ \\
\hline
\end{tabular}

Table 14: Experimental parameters of the GE $3 \times 3$ tests

\begin{tabular}{|l|l|}
\hline Name of the test & GE $\mathbf{3 x 3}$ \\
\hline $\begin{array}{l}\text { Reference in } \\
\text { literature }\end{array}$ & {$[6]$} \\
\hline $\begin{array}{l}\text { Measured } \\
\text { quantities }\end{array}$ & $\begin{array}{l}\text { Mixing tests, yeilding: } \\
\text { mass flow rate } \\
\text { equilibrium quality at the bundle outlet } \\
\text { for the total bundle, } \\
\text { for 3 subchannels each representing a characteristic class } \\
\text { of subchannels (corner, side, central) in non-simultaneous } \\
\text { sampling - only one subchannel sampled at a certain measurement }\end{array}$ \\
\hline $\begin{array}{l}\text { Measuring } \\
\text { uncertainties }\end{array}$ & $\begin{array}{l}\text { Total bundle mass flow rate: } \pm 8 \% \\
\text { Subchannel flow rate and subchannel enthalpy: } \pm 3 \%\end{array}$ \\
\hline
\end{tabular}


Table 15: GE 3x3 Test Points

\begin{tabular}{|c|c|c|c|c|}
\hline $\begin{array}{c}\text { Number of } \\
\text { test point }\end{array}$ & $\begin{array}{c}\text { Total inlet mass } \\
\text { flow rate } \\
{[\mathrm{kg} / \mathrm{s}]}\end{array}$ & $\begin{array}{c}\text { Inlet enthalpy } \\
{[\mathrm{kJ} / \mathrm{kg}]}\end{array}$ & $\begin{array}{c}\text { Total rod } \\
\text { power } \\
{[\mathrm{kW}]}\end{array}$ & $\begin{array}{c}\text { Exit pressure } \\
{[\mathrm{bar}]}\end{array}$ \\
\hline 1B & 1.231 & 93.7 & 0 & 70 \\
\hline 1C & 2.538 & 93.7 & 0 & 70 \\
\hline 1D & 3.871 & 93.7 & 0 & 70 \\
\hline 1E & 5.050 & 93.7 & 0 & 70 \\
\hline 2B2 & 1.359 & 918.7 & 532 & 70 \\
\hline 2B3 & 1.372 & 1014.6 & 532 & 70 \\
\hline 2B4 & 1.372 & 1144.6 & 532 & 70 \\
\hline 2C1 & 2.717 & 1134.4 & 532 & 70 \\
\hline 2C2 & 2.738 & 1185.8 & 532 & 70 \\
\hline 2D1 & 1.384 & 664.5 & 1064 & 70 \\
\hline 2D3 & 1.384 & 978.1 & 1064 & 70 \\
\hline 2E1 & 2.769 & 936.2 & 1064 & 70 \\
\hline 2E2 & 2.769 & 1042.5 & 1064 & 70 \\
\hline 2E3 & 2.717 & 1199.7 & 1064 & 70 \\
\hline 2G1 & 2.743 & 742.0 & 1596 & 70 \\
\hline 2G2 & 2.769 & 825.9 & 1596 & 70 \\
\hline 2G3 & 2.743 & 926.2 & 1596 & 70 \\
\hline 3B2 & 1.372 & 1237.4 & 532 & 70 \\
\hline 3D1 & 1.397 & 2474.9 & 1064 & 70 \\
\hline 3E1 & 2.769 & 2474.9 & 1064 & 70 \\
\hline 3E2 & 2.717 & 2312.0 & 994 & 70 \\
\hline
\end{tabular}




\section{A.2 CTF Model of the GE 3x3 Test Section}

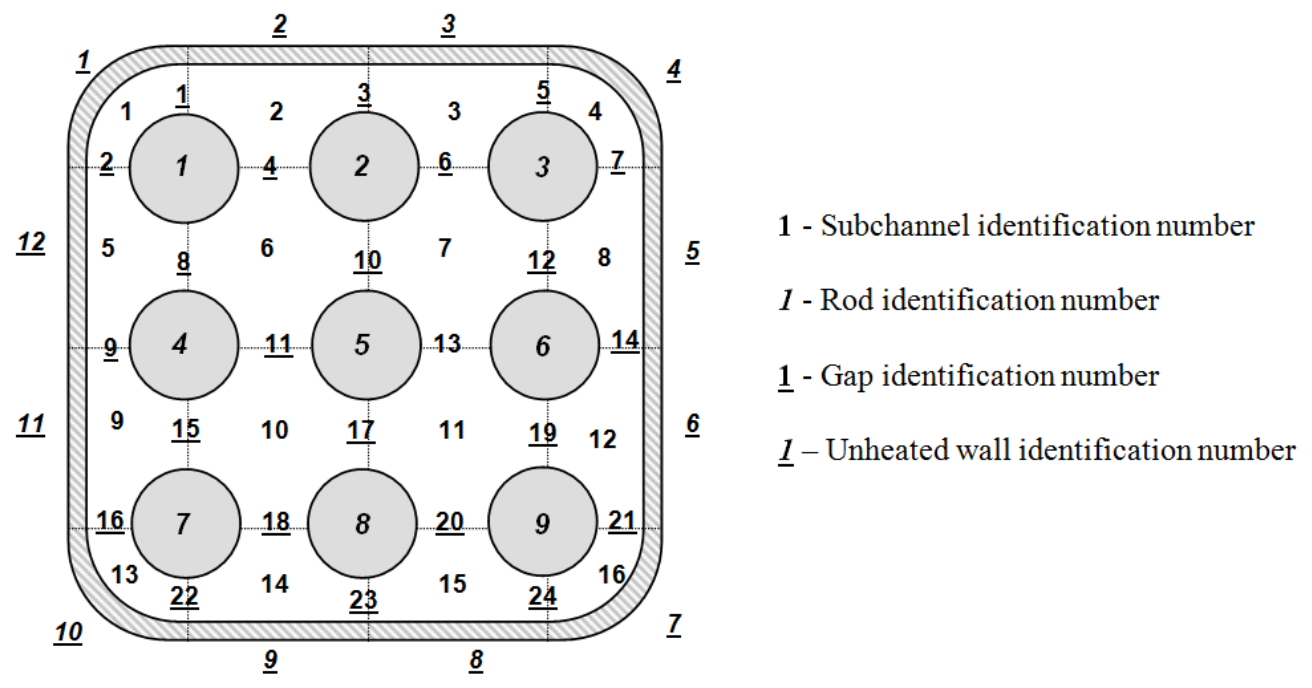

Figure A.1: Cross section of the CTF model of the GE $3 \times 3$ rod bundle 
Table 16: CTF modeling parameters for the GE 3x3 Tests

\begin{tabular}{|c|c|}
\hline Units in input file & US units \\
\hline Entrainment / deposition model & $\begin{array}{l}\text { Original CTF model (Paleev and Filippovich correla- } \\
\text { tion for entrainment, Cousins model for deposition) }\end{array}$ \\
\hline $\begin{array}{l}\text { Direct heating of the coolant (by neutron mod- } \\
\text { eration) }\end{array}$ & none \\
\hline Initial pressure of the fluid & equal to the outlet pressure \\
\hline Initial enthalpy of the fluid & equal to the inlet enthalpy \\
\hline Heat loss to ambient & none \\
\hline Initial rod temperature & $\begin{array}{l}149 \hat{\mathrm{A}} \check{\mathrm{r} C}(137 \mathrm{~K} \text { below the saturation temperature at } \\
70 \text { bars) }\end{array}$ \\
\hline Radiation & none \\
\hline Forcing functions for rod power & $\begin{array}{l}\text { Test points 1B-1E: noneTest points 2B2-2G3: } \\
\text { noneTest points 3B2-3E2: non-uniform radial peaking } \\
\text { pattern (but not time-dependent) }\end{array}$ \\
\hline Mixing model & $\begin{array}{l}\text { Standard mixing and void drift model according to } \\
\text { user specified mixing coefficient BETA. BETA }=0.02 \text {, } \\
\mathrm{KM}=1.4\end{array}$ \\
\hline Boundary condition type & $\begin{array}{l}\text { Inlet: mass flow rate and enthalpy|Outlet: pressure } \\
\text { and enthalpy }\end{array}$ \\
\hline Simulation time & $10 \mathrm{~s}$ to reach stationary conditions \\
\hline No. of test points in the calculation & 21 \\
\hline
\end{tabular}

\section{A.3 CTF Input Deck for the GE 3x3 Test Point 2B2}

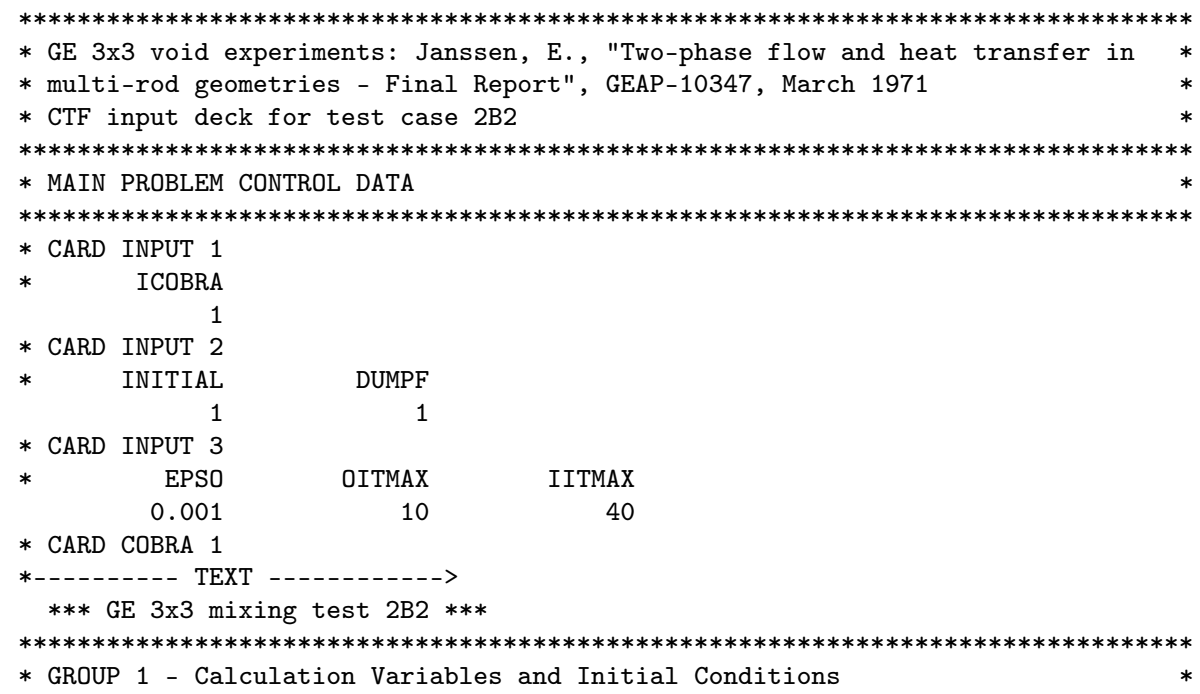


* CARD GROUP 1

* NGR

1

* Card 1.1

* NGAS IRFC EDMD IMIX ISOL NDM6 NDM7 NDM8 NDM9 NM10 NM11 NM12 NM13 NM14

$\begin{array}{llllllllllllll}1 & 1 & 1 & 2 & 0 & 0 & 0 & 0 & 0 & 0 & 0 & 0 & 0 & 0\end{array}$

* Card 1.2

* GTOT AFLUX DHFRAC

$\begin{array}{rrr}1.359 & 32.322 & 0.0\end{array}$

* Card 1.3

* PREF HIN HGIN VFRAC1 VFRAC2

$\begin{array}{lllll}70.00 & 918.77 & 288.4 & 1.0 & 0.9999\end{array}$

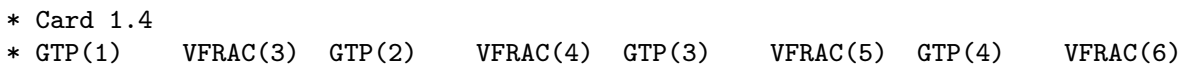

$\begin{array}{rr}* \text { GTP(1) } & \text { air } \\ * * * * * * * * * * * * * * * * *\end{array}$

$* * * * * * * * * * * * * * * * * * * * * * * * * * * * * * * * * * * * * * * * * * * * * * * * * * * * * * * * * * * * * * * * * * * * * * * * * * * * * * * * * * * *$

* GROUP 2.0 - Channel Description

$* * * * * * * * * * * * * * * * * * * * * * * * * * * * * * * * * * * * * * * * * * * * * * * * * * * * * * * * * * * * * * * * * * * * * * * * * * * * * * * * * * * * *$

$*$ CARD GROUP 2

* NGR

2

* Card 2.1

* NCHA NDM2 NDM3 NDM4 NDM5 NDM6 NDM7 NDM8 NDM9 NM10 NM11 NM12 NM13 NM14

$\begin{array}{llllllllllllll}16 & 0 & 0 & 0 & 0 & 0 & 0 & 0 & 0 & 0 & 0 & 0 & 0 & 0\end{array}$

* Card 2.2

* I AN PW ABOT ATOP NMGP

$\begin{array}{llllll}1 & 0.00005050 & 0.02834629 & 0.0 & 0.0 & 0.0\end{array}$

$\begin{array}{llllll}2 & 0.00011766 & 0.04148719 & 0.0 & 0.0 & 0.0\end{array}$

$\begin{array}{llllll}3 & 0.00011766 & 0.04148719 & 0.0 & 0.0 & 0.0\end{array}$

$\begin{array}{llllll}4 & 0.00005050 & 0.02834629 & 0.0 & 0.0 & 0.0\end{array}$

$\begin{array}{llllll}5 & 0.00011766 & 0.04148719 & 0.0 & 0.0 & 0.0\end{array}$

$\begin{array}{llllll}6 & 0.00018675 & 0.04548398 & 0.0 & 0.0 & 0.0\end{array}$

$\begin{array}{llllll}7 & 0.00018675 & 0.04548398 & 0.0 & 0.0 & 0.0\end{array}$

$\begin{array}{llllll}8 & 0.00011766 & 0.04148719 & 0.0 & 0.0 & 0.0\end{array}$

$\begin{array}{llllll}9 & 0.00011766 & 0.04148719 & 0.0 & 0.0 & 0.0\end{array}$

$\begin{array}{llllll}10 & 0.00018675 & 0.04548398 & 0.0 & 0.0 & 0.0\end{array}$

$\begin{array}{llllll}11 & 0.00018675 & 0.04548398 & 0.0 & 0.0 & 0.0\end{array}$

$\begin{array}{llllll}12 & 0.00011766 & 0.04148719 & 0.0 & 0.0 & 0.0\end{array}$

$\begin{array}{llllll}13 & 0.00005050 & 0.02834629 & 0.0 & 0.0 & 0.0\end{array}$

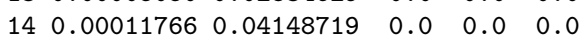

$\begin{array}{llllll}15 & 0.00011766 & 0.04148719 & 0.0 & 0.0 & 0.0\end{array}$

$\begin{array}{llllll}16 & 0.00005050 & 0.02834629 & 0.0 & 0.0 & 0.0\end{array}$

$*$

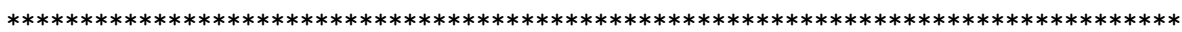

* GROUP 3.0 - Transverse Channel Connection (Gap) Data

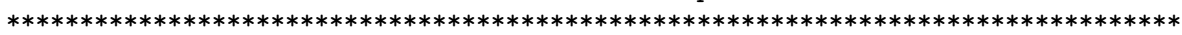

$*$ CARD GROUP 3

* NGR

3

* Card 3.1

* NK NDM2 NDM3 NDM4 NDM5 NDM6 NDM7 NDM8 NDM9 NM10 NM11 NM12 NM13 NM14
240

* Card 3.2

* $\mathrm{K} \quad \mathrm{IK}$

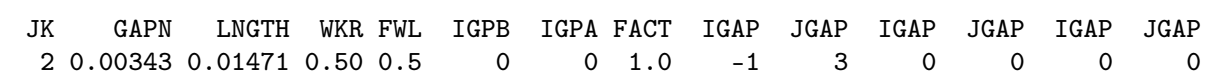

* Card 3.3

* GMLT ETNR

1.0000 .000

* Cards 3.2 and 3.3 continued 


\begin{tabular}{|c|c|c|c|c|c|c|c|c|c|c|c|c|c|c|c|}
\hline 2 & 1 & 5 & 50.00343 & 0.01471 & 0.50 & 0.5 & 0 & 0 & 1.0 & -1 & 9 & 0 & 0 & 0 & 0 \\
\hline 1.000 & 0.000 & & & & & & & & & & & & & & \\
\hline 3 & 2 & 3 & 30.00343 & 0.01875 & 0.50 & 0.5 & 0 & 0 & 1.0 & 1 & 5 & 0 & 0 & 0 & 0 \\
\hline 1.000 & 0.000 & & & & & & & & & & & & & & \\
\hline 4 & 2 & 6 & 60.00427 & 0.01471 & 0.50 & 0.0 & 0 & 0 & 1.0 & -1 & 11 & 0 & o & 0 & 0 \\
\hline 1.000 & 0.000 & & & & & & & & & & & & & & \\
\hline 5 & 3 & 4 & 40.00343 & 0.01471 & 0.50 & 0.5 & 0 & 0 & 1.0 & 3 & -1 & 0 & 0 & 0 & 0 \\
\hline 1.000 & 0.000 & & & & & & & & & & & & & & \\
\hline 6 & 3 & 7 & 70.00427 & 0.01471 & 0.50 & 0.0 & 0 & 0 & 1.0 & -1 & 13 & 0 & c & 0 & 0 \\
\hline 1.000 & 0.000 & & & & & & & & & & & & & & \\
\hline 7 & 4 & 8 & 80.00343 & 0.01471 & 0.50 & 0.5 & 0 & 0 & 1.0 & -1 & 14 & 0 & 0 & 0 & 0 \\
\hline 1.000 & 0.000 & & & & & & & & & & & & & & \\
\hline 8 & 5 & 6 & 60.00427 & 0.01471 & 0.50 & 0.0 & 0 & 0 & 1.0 & -1 & 10 & 0 & 0 & 0 & 0 \\
\hline 1.000 & 0.000 & & & & & & & & & & & & & & \\
\hline 9 & 5 & 9 & 90.00343 & 0.01875 & 0.50 & 0.5 & 0 & 0 & 1.0 & 2 & 16 & 0 & 0 & 0 & 0 \\
\hline 1.000 & 0.000 & & & & & & & & & & & & & & \\
\hline 10 & 6 & 7 & 70.00427 & 0.01875 & 0.50 & 0.0 & 0 & 0 & 1.0 & 8 & 12 & 0 & 0 & 0 & 0 \\
\hline 1.000 & 0.000 & & & & & & & & & & & & & & \\
\hline 11 & 6 & 10 & 00.00427 & 0.01875 & 0.50 & 0.0 & 0 & 0 & 1.0 & 4 & 18 & 0 & 0 & 0 & 0 \\
\hline 1.000 & 0.000 & & & & & & & & & & & & & & \\
\hline 12 & 7 & 8 & 80.00427 & 0.01471 & 0.50 & 0.0 & 0 & 0 & 1.0 & 10 & -1 & 0 & 0 & 0 & 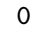 \\
\hline 1.000 & 0.000 & & & & & & & & & & & & & & \\
\hline 13 & 7 & 11 & 10.00427 & 0.01875 & 0.50 & 0.0 & 0 & 0 & 1.0 & 6 & 20 & 0 & 0 & 0 & \\
\hline 1.000 & 0.000 & & & & & & & & & & & & & & \\
\hline 14 & 8 & 12 & 20.00343 & 0.01875 & 0.50 & 0.5 & 0 & 0 & 1.0 & 7 & 21 & 0 & 0 & 0 & 0 \\
\hline 1.000 & 0.000 & & & & & & & & & & & & & & \\
\hline 15 & 9 & 10 & 0.00427 & 0.01471 & 0.50 & 0.0 & 0 & 0 & 1.0 & -1 & 17 & 0 & 0 & 0 & 0 \\
\hline 1.000 & 0.000 & & & & & & & & & & & & & & \\
\hline 16 & 9 & 13 & 30.00343 & 0.01471 & 0.50 & 0.5 & 0 & 0 & 1.0 & 9 & -1 & 0 & 0 & 0 & 0 \\
\hline 1.000 & 0.000 & & & & & & & & & & & & & & \\
\hline 17 & 10 & 11 & 10.00427 & 0.01875 & 0.50 & 0.0 & 0 & 0 & 1.0 & 15 & 19 & 0 & 0 & 0 & 0 \\
\hline 1.000 & 0.000 & & & & & & & & & & & & & & \\
\hline 18 & 10 & 14 & 40.00427 & 0.01471 & 0.50 & 0.0 & 0 & 0 & 1.0 & 11 & -1 & 0 & o & 0 & 0 \\
\hline 1.000 & 0.000 & & & & & & & & & & & & & & \\
\hline 19 & 11 & 12 & 20.00427 & 0.01471 & 0.50 & 0.0 & 0 & 0 & 1.0 & 17 & -1 & 0 & 0 & 0 & 0 \\
\hline 1.000 & 0.000 & & & & & & & & & & & & & & \\
\hline 20 & 11 & 15 & 50.00427 & 0.01471 & 0.50 & 0.0 & 0 & 0 & 1.0 & 13 & -1 & 0 & o & 0 & 0 \\
\hline 1.000 & 0.000 & & & & & & & & & & & & & & \\
\hline 21 & 12 & 16 & 60.00343 & 0.01471 & 0.50 & 0.5 & 0 & 0 & 1.0 & 14 & -1 & 0 & c & 0 & 0 \\
\hline 1.000 & 0.000 & & & & & & & & & & & & & & \\
\hline 22 & 13 & 14 & 40.00343 & 0.01471 & 0.50 & 0.5 & 0 & 0 & 1.0 & -1 & 23 & 0 & 0 & 0 & 0 \\
\hline 1.000 & 0.000 & & & & & & & & & & & & & & \\
\hline 23 & 14 & 15 & 50.00343 & 0.01875 & 0.50 & 0.5 & 0 & 0 & 1.0 & 22 & 24 & 0 & o & 0 & 0 \\
\hline 1.000 & 0.000 & & & & & & & & & & & & & & \\
\hline 24 & 15 & 16 & 60.00343 & 0.01471 & 0.50 & 0.5 & 0 & 0 & 1.0 & 23 & -1 & 0 & 0 & 0 & \\
\hline 1.000 & 0.000 & & & & & & & & & & & & & & \\
\hline & & & & & & & & & & & & & & & \\
\hline * Card & d 3.4 & & & & & & & & & & & & & & \\
\hline * NLGP & & & & & & & & & & & & & & & \\
\hline 0 & & & & & & & & & & & & & & & \\
\hline * & & & & & & & & & & & & & & & \\
\hline$* * * * * *$ & $* * * * * * *$ & $* * * * * *$ & $* * * * * * * * * *$ & $k * * * * * * *$ & $* * * * *$ & $* * * * * * * * *$ & $* * * *$ & & & $* * * *$ & $* *$ & $<* *$ & & & \\
\hline * GROU & UP 4.0 & - Ver & rtical Cha & annel Cor & nnect: & ion Data & & & & & & & & & \\
\hline$* * * * * *$ & $* * * * * * *$ & $* * * * * *$ & $* * * * * * * * * *$ & $k * * * * * * *$ & & $* * * * * * * * *$ & $* * * *$ & $* * * * *$ & $* * * * *$ & $* * * * *$ & $* * * * *$ & $* * * *$ & & & \\
\hline * CARD & D GROUP & 4 & & & & & & & & & & & & & \\
\hline * NGR & & & & & & & & & & & & & & & \\
\hline 4 & & & & & & & & & & & & & & & \\
\hline * Card & d 4.1 & & & & & & & & & & & & & & \\
\hline * NSEC & C NSIM & IREB & NDM4 NDM5 & 5 NDM6 $\mathrm{NI}$ & DM7 NI & DM8 NDM9 & NM10 & NM11 & 1 NM12 & NM13 & NM14 & & & & \\
\hline 1 & 11 & 0 & $\begin{array}{ll}0 & 0\end{array}$ & 0 & 0 & $0 \quad 0$ & 0 & & $0 \quad 0$ & 0 & 0 & & & & \\
\hline
\end{tabular}


* Card 4.2

* ISEC NCHN NONO DXS IVAR

* Card 4.3

* I KCHA KCHA KCHA KCHA KCHA KCHA KCHB KCHB KCHB KCHB KCHB KCHB

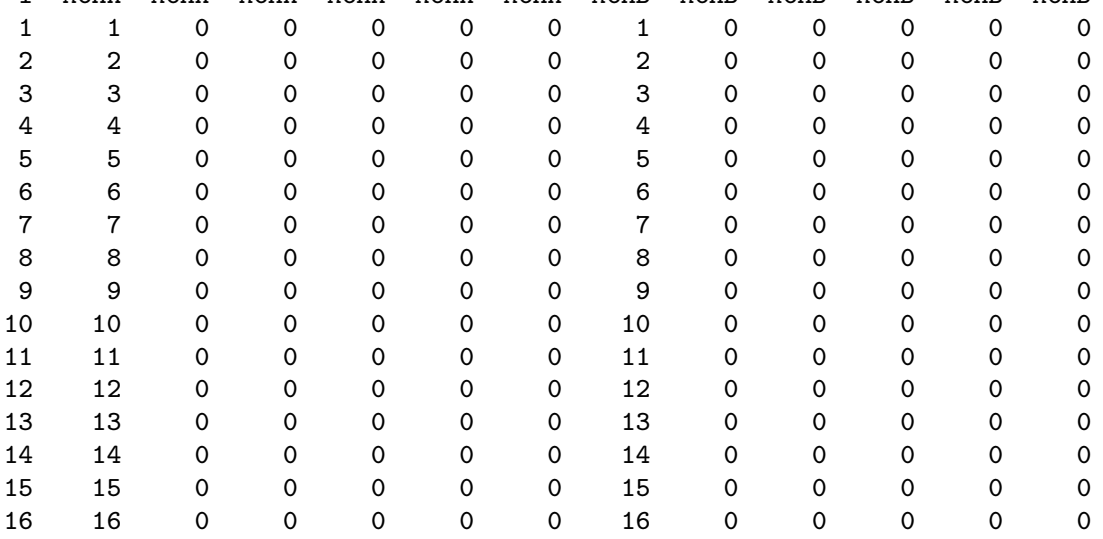

* Card 4.5

* IWDE

16

* Card 4.6

* MSIM

576

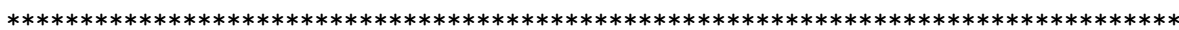

* GROUP 7 - Local Loss Coefficient and Grid Spacer Data

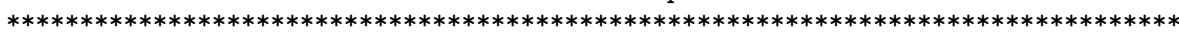

$*$ CARD GROUP 7

* NGR

7

* Card 7.1

* NCD NGT IFGQ IFSD IFES IFTP IGTM NFBS NDM9 NM10 NM11 NM12 NM13 NM14 18
Card 7.2

* CDL $\begin{array}{lll}* & \text { J ICDM }\end{array}$

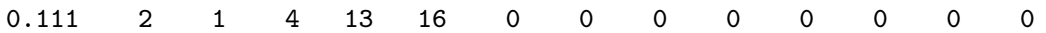

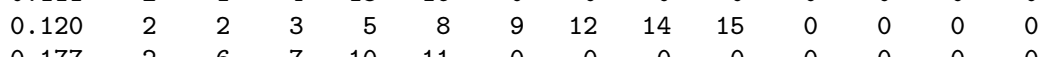

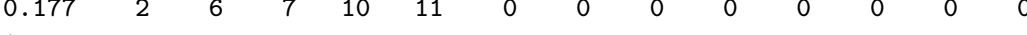

$\begin{array}{llllllllllllll}0.111 & 8 & 1 & 4 & 13 & 16 & 0 & 0 & 0 & 0 & 0 & 0 & 0 & 0\end{array}$

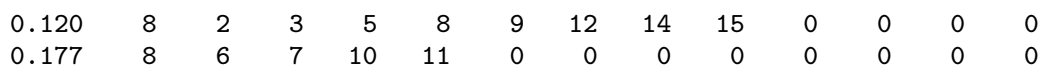

*

$\begin{array}{llllllllllllll}0.111 & 14 & 1 & 4 & 13 & 16 & 0 & 0 & 0 & 0 & 0 & 0 & 0 & 0\end{array}$

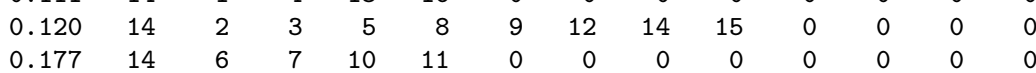

*

$\begin{array}{llllllllllllll}0.111 & 20 & 1 & 4 & 13 & 16 & 0 & 0 & 0 & 0 & 0 & 0 & 0 & 0\end{array}$

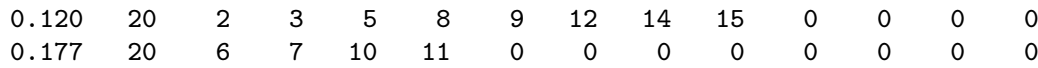

*

$\begin{array}{llllllllllllll}0.111 & 26 & 1 & 4 & 13 & 16 & 0 & 0 & 0 & 0 & 0 & 0 & 0 & 0\end{array}$

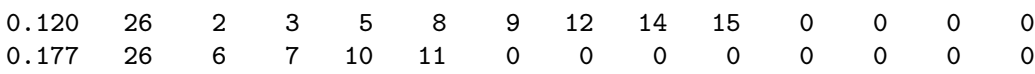

*

$\begin{array}{llllllllllllll}0.111 & 32 & 1 & 4 & 13 & 16 & 0 & 0 & 0 & 0 & 0 & 0 & 0 & 0\end{array}$ 


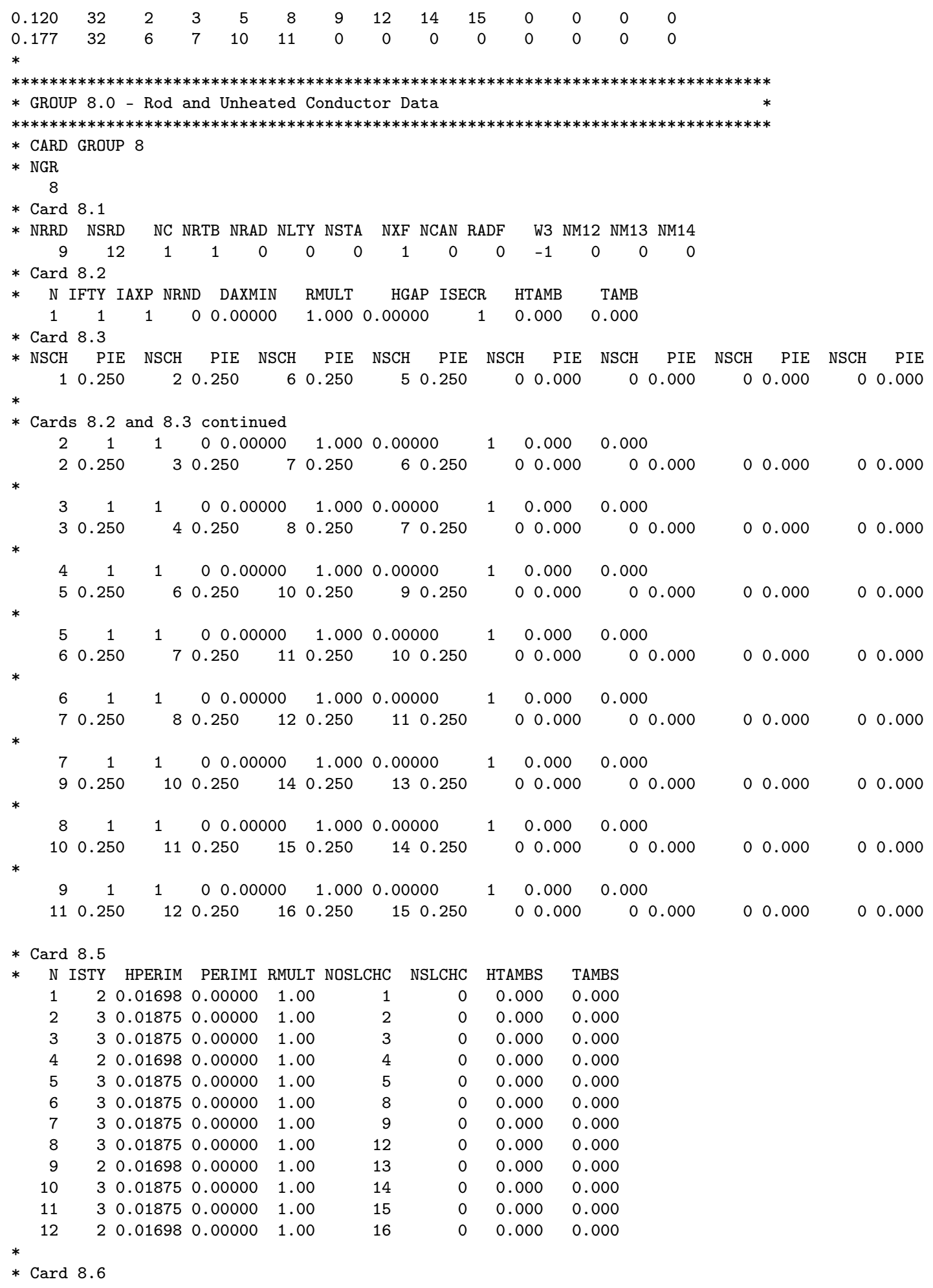


* I NRT1 NST1 NRX1

$*$

* Card 8.7

* IRTAB IRTAB IRTAB IRTAB IRTAB IRTAB IRTAB IRTAB IRTAB IRTAB IRTAB IRTAB

* Card 8.8

* IRTAB IRTAB IRTAB IRTAB IRTAB IRTAB IRTAB IRTAB IRTAB IRTAB IRTAB IRTAB $\begin{array}{llllllllllll}1 & 2 & 3 & 4 & 5 & 6 & 7 & 8 & 9 & 10 & 11 & 12\end{array}$

$*$

* Card 8.9

* AXIALT TRINIT

$0.0000000 \quad 300.00000$

1.8300000300 .00000

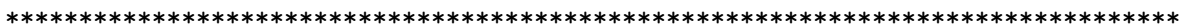

* GROUP 9.0 - Conductor Geometry Description

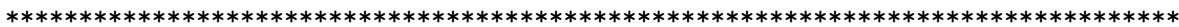

* CARD GROUP 9

* NGR

9

* Card 9.1

* NFLT IRLF ICNF IMWR NDM5 NDM6 NDM7 NDM8 NDM9 NM10 NM11 NM12 NM13 NM14

* Card 9.6

* I FTYP DROD DIN NFUL ITOX ITIX NDM8 NDM9 NM10 NM11 NM12 NM13 NM14

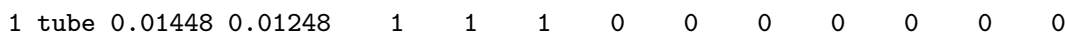

* Card 9.7

* NODR MATR TREG QREG

210.001001 .00000

$*$

* Card 9.6

* I FTYP DROD DIN NFUL ITOX ITIX NDM8 NDM9 NM10 NM11 NM12 NM13 NM14

2 wall 0.016980 .00100

* Card 9.7

* NODR MATR TREG QREG

210.001000 .00000

$*$

* Card 9.6

* I FTYP DROD DIN NFUL ITOX ITIX NDM8 NDM9 NM10 NM11 NM12 NM13 NM14

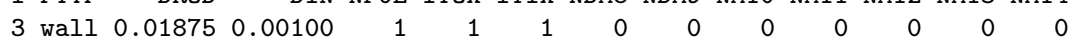

* Card 9.7

* NODR MATR TREG QREG $210.00100 \quad 0.00000$

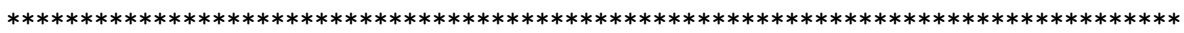

* GROUP 10 - Material Properties Tables

$* * * * * * * * * * * * * * * * * * * * * * * * * * * * * * * * * * * * * * * * * * * * * * * * * * * * * * * * * * * * * * * * * * * * * * * * * * * * * * * * * *$

$*$ CARD GROUP 10

* NGR

10

* Card 10.1

* NMAT NDM2 NDM3 NDM4 NDM5 NDM6 NDM7 NDM8 NDM9 NM10 NM11 NM12 NM13 NM14

* Card 10.2

* N NTDP RCOLD

$\begin{array}{lll}1 & 6 & 8470.57\end{array}$

* Card 10.3 


$\begin{array}{rrr}\text { * TPROP } & \text { CPF1 } & \text { THCF } \\ -73 & 0.377 & 13.40 \\ 93 & 0.464 & 15.71 \\ 204 & 0.485 & 17.44 \\ 427 & 0.527 & 20.90 \\ 649 & 0.586 & 24.79 \\ 871 & 0.623 & 28.83\end{array}$

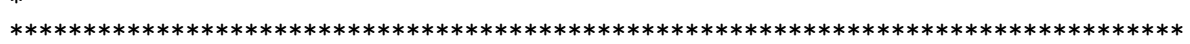

* GROUP 11.0 - Axial Power Tables and Forcing Functions

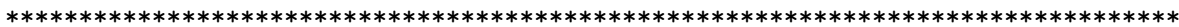

* CARD GROUP 11

* NGR

11

* Card 11.1

* NQA NAXP MNXN NQ NGPF NQR NDM7 NDM8 NDM9 NM10 NM11 NM12 NM13 NM14

$*$

* Axial Power Forcing Functions

* Card 11.2

* YQA

0.0

* Card 11.3

* I NAXN

12

* Card 11.4

* Y

$0.000000 \quad 1.000000$

$1.830000 \quad 1.000000$

$*$

* Total Power Forcing Functions

* Card 11.5

* YQ FQ

* $0.0000 \quad 0.0000$

* $1.0000 \quad 1.0000$

* $100.0000 \quad 1.0000$

$*$

* Radial Power Forcing Functions

* Card 11.7

* YQR

$$
0.0
$$

* Card 11.8

* FQR

1.0000

FQR FQR

1.0000

FQR

FQR
1.0000

FQR
1.0000

FQR
1.0000

FQR

1.0000

$*$

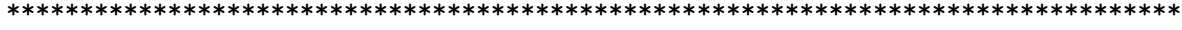

* GROUP 12 - Turbulent mixing data

$* * * * * * * * * * * * * * * * * * * * * * * * * * * * * * * * * * * * * * * * * * * * * * * * * * * * * * * * * * * * * * * * * * * * * * * * * * * * * * * * * * *$

* CARD GROUP 12

* NGR

12

* Card 12.2

* AAAK DFRD THEM

$\begin{array}{lll}1.4 & 0.01448 \quad 5.0\end{array}$

$*$

$* * * * * * * * * * * * * * * * * * * * * * * * * * * * * * * * * * * * * * * * * * * * * * * * * * * * * * * * * * * * * * * * * * * * * * * * * * * * * * * * * * *$

* GROUP 13.0 - Boundary Condition Data

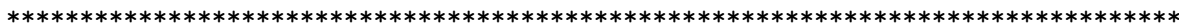

$*$ CARD GROUP 13 
* Card 13.1

* NBND NKBD NFUN NGBD NDM5 NDM6 NDM7 NDM8 NDM9 NM10 NM11 NM12 NM13 NM14

* Card 13.2

* NPT

$$
4
$$

* Card 13.3

* ABSC ORDINT ABSC ORDINT ABSC ORDINT
$0.0 \quad 0.000$
0.10 .000
$0.2 \quad 1.0001500 .0 \quad 1.000$

* Card 13.4

* Inlet b.c. --

* IBD1 IBD2 ISPC N1FN N2FN N3FN BCVALUE1 BCVALUE2 BCVALUE3 INITGAS

$\begin{array}{rrrrrrrrrr}1 & 1 & 2 & 1 & 0 & 0 & 0.0 & 918.77 & 0.0000 & 1 \\ 2 & 1 & 2 & 1 & 0 & 0 & 0.0 & 918.77 & 0.0000 & 1 \\ 3 & 1 & 2 & 1 & 0 & 0 & 0.0 & 918.77 & 0.0000 & 1 \\ 4 & 1 & 2 & 1 & 0 & 0 & 0.0 & 918.77 & 0.0000 & 1 \\ 5 & 1 & 2 & 1 & 0 & 0 & 0.0 & 918.77 & 0.0000 & 1 \\ 6 & 1 & 2 & 1 & 0 & 0 & 0.0 & 918.77 & 0.0000 & 1 \\ 7 & 1 & 2 & 1 & 0 & 0 & 0.0 & 918.77 & 0.0000 & 1 \\ 8 & 1 & 2 & 1 & 0 & 0 & 0.0 & 918.77 & 0.0000 & 1 \\ 9 & 1 & 2 & 1 & 0 & 0 & 0.0 & 918.77 & 0.0000 & 1 \\ 10 & 1 & 2 & 1 & 0 & 0 & 0.0 & 918.77 & 0.0000 & 1 \\ 11 & 1 & 2 & 1 & 0 & 0 & 0.0 & 918.77 & 0.0000 & 1 \\ 12 & 1 & 2 & 1 & 0 & 0 & 0.0 & 918.77 & 0.0000 & 1 \\ 13 & 1 & 2 & 1 & 0 & 0 & 0.0 & 918.77 & 0.0000 & 1 \\ 14 & 1 & 2 & 1 & 0 & 0 & 0.0 & 918.77 & 0.0000 & 1 \\ 15 & 1 & 2 & 1 & 0 & 0 & 0.0 & 918.77 & 0.0000 & 1 \\ 16 & 1 & 2 & 1 & 0 & 0 & 0.0 & 918.77 & 0.0000 & 1\end{array}$

* Outlet b.c. -

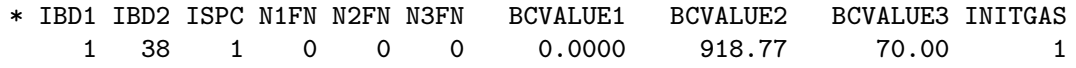

$\begin{array}{rlllllllll}1 & 38 & 1 & 0 & 0 & 0 & 0.0000 & 918.77 & 70.00 & 1 \\ 2 & 38 & 1 & 0 & 0 & 0 & 0.0000 & 918.77 & 70.00 & 1 \\ 3 & 38 & 1 & 0 & 0 & 0 & 0.0000 & 918.77 & 70.00 & 1 \\ 4 & 38 & 1 & 0 & 0 & 0 & 0.0000 & 918.77 & 70.00 & 1 \\ 5 & 38 & 1 & 0 & 0 & 0 & 0.0000 & 918.77 & 70.00 & 1 \\ 6 & 38 & 1 & 0 & 0 & 0 & 0.0000 & 918.77 & 70.00 & 1 \\ 7 & 38 & 1 & 0 & 0 & 0 & 0.0000 & 918.77 & 70.00 & 1 \\ 8 & 38 & 1 & 0 & 0 & 0 & 0.0000 & 918.77 & 70.00 & 1 \\ 9 & 38 & 1 & 0 & 0 & 0 & 0.0000 & 918.77 & 70.00 & 1 \\ 10 & 38 & 1 & 0 & 0 & 0 & 0.0000 & 918.77 & 70.00 & 1 \\ 11 & 38 & 1 & 0 & 0 & 0 & 0.0000 & 918.77 & 70.00 & 1 \\ 12 & 38 & 1 & 0 & 0 & 0 & 0.0000 & 918.77 & 70.00 & 1 \\ 13 & 38 & 1 & 0 & 0 & 0 & 0.0000 & 918.77 & 70.00 & 1 \\ 14 & 38 & 1 & 0 & 0 & 0 & 0.0000 & 918.77 & 70.00 & 1 \\ 15 & 38 & 1 & 0 & 0 & 0 & 0.0000 & 918.77 & 70.00 & 1 \\ 16 & 38 & 1 & 0 & 0 & 0 & 0.0000 & 918.77 & 70.00 & 1\end{array}$

$*$

$* * * * * * * * * * * * * * * * * * * * * * * * * * * * * * * * * * * * * * * * * * * * * * * * * * * * * * * * * * * * * * * * * * * * * * * * * * * * * * * * * * * * *$

* Group 14 - Output Options

$* * * * * * * * * * * * * * * * * * * * * * * * * * * * * * * * * * * * * * * * * * * * * * * * * * * * * * * * * * * * * * * * * * * * * * * * * * * * * * * * * * *$

$*$ NGRP

14

* N1 NOU1 NOU2 NOU3 NOU4 IPRP IOPT IRWR NDM9 NM10 NM11 NM12 NM13 NM14

$\begin{array}{rrrrr} & 5 & 0 & 0 & 0 \\ * \text { *PRCH } & & & \\ * & 5 & 13 & 17 & 20 \\ * & 9 & 25 & 36 & \end{array}$




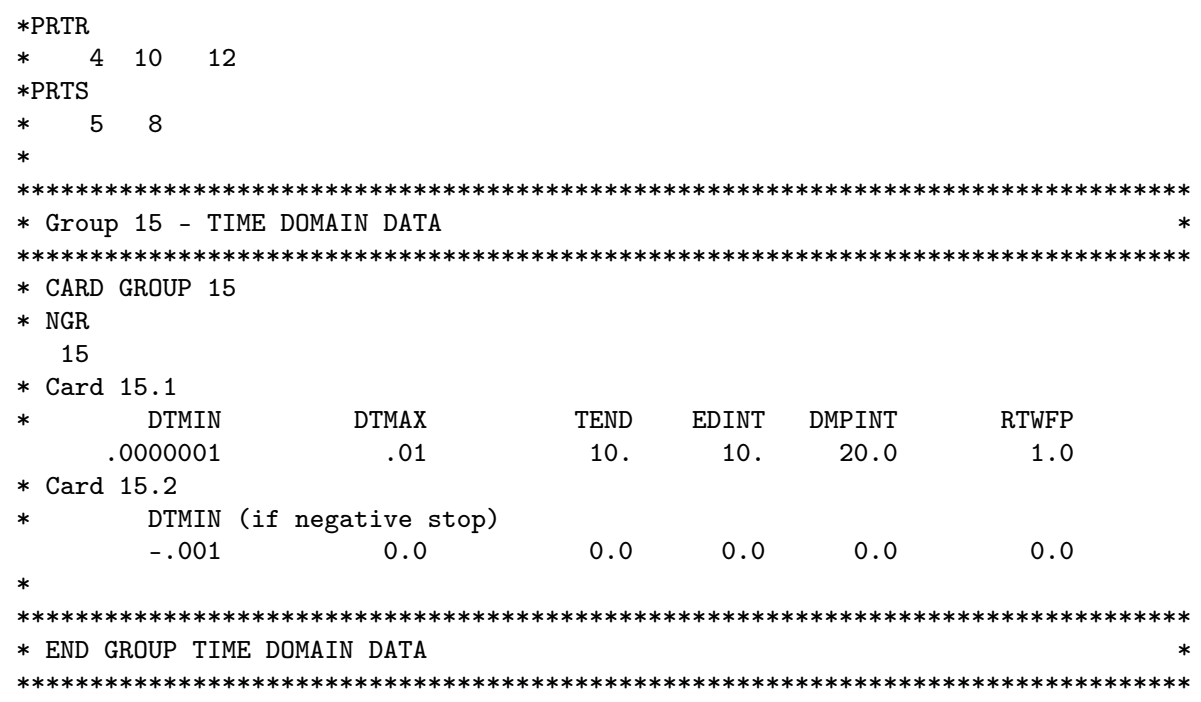




\section{Bibliography}

[1] R. Salko et al. CTF Theory Manual. Tech. rep. CASL-2019-1886-001. 2019.

[2] S.G. Beus. A Two-Phase Turbulent Mixing Model for Flow in Rod Bundles. Tech. rep. WAPD-/TM2438. Bettis Atomic Power Laboratory, 1971.

[3] M. J. Thurgood et al. COBRA/TRAC-A Thermal-Hydraulics Code for Transient Analysis of Nuclear Reactor Vessels and Primary Coolant Systems. Tech. rep. NUREG/CR-3046. Pacific Northwest Laboratory, 1983.

[4] C.Y. Paik et al. Analysis of FLECHT-SEASET 163-Rod Blocked Bundle Data using COBRA-TF. Tech. rep. NUREG/CR-3046. United States Nuclear Regulatory Commission, 1985.

[5] M. Yilmaz. "Development of Burnup Dependent Fuel Rod Model in CTF". PhD thesis. The Pennsylvania State University, 2014.

[6] D. W. Radcliffe R. T. Lahey Jr. B. S. Shirlakar. Two-Phase Flow and Heat Transfer In Multirod Geometries: Subchannel and Pressure Drop Measurements in a Nine-Rod Bundle for Diabatic and Adiabatic Conditions. Tech. rep. General Electric, 1970. 